\title{
Impact of stroke and enriched environment on visual cortical plasticity in mice and therapeutic interventions for rehabilitation
}

\author{
Dissertation \\ for the award of the degree \\ "Doctor rerum naturalium" \\ of the Georg-August-Universität Göttingen
}

\begin{abstract}
within the doctoral program Sensory and Motor Neuroscience
of the Göttingen Graduate School for Neurosciences, Biophysics, and Molecular

Biosciences (GGNB)

of the Georg-August University School of Science (GAUSS)
\end{abstract}

submitted by

Franziska Greifzu

from Gera, Germany

Göttingen 2013 
$\underline{\text { Examination committee }}$

Prof. Dr. Siegrid Löwel

( $1^{\text {st }}$ Reviewer, advisor, member of the thesis committee)

Systems Neuroscience Group

Johann-Friedrich-Blumenbach-Institute for Zoology und Anthropology

Bernstein Focus for Neurotechnology

University of Göttingen

\section{Prof. Dr. Andrea Antal}

( $2^{\text {nd }}$ Reviewer, member of the thesis committee)

Department of Clinical Neurophysiology

University of Göttingen School of Medicine

\section{Prof. Dr. Fred Wolf}

(Member of the thesis committee)

Research Group Theoretical Neurophysics

Department of Nonlinear Dynamics

Max Planck Institute for Dynamics and Self-Organization, Göttingen

\section{Prof. Dr. Fred Wolf}

(Member of the thesis committee)

Research Group Theoretical Neurophysics

Department of Nonlinear Dynamics

Max Planck Institute for Dynamics and Self-Organization, Göttingen

\section{Prof. Dr. Tim Gollisch}

Systems Neuroscience Group

Department of Opthalmology

University of Göttingen School of Medicine

\section{Prof. Dr. André Fischer}

European Neuroscience Institute

Göttingen

\section{Prof. Dr. Andreas Stumpner}

Cellular Neurobiology

Schwann-Schleiden-Forschungszentrum

University of Göttingen

Date of oral examination: 19.04.2013 


\section{TABLE OF CONTENTS}

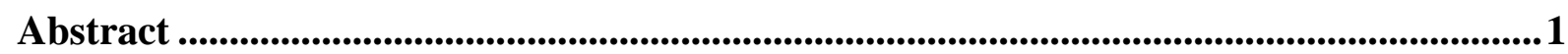

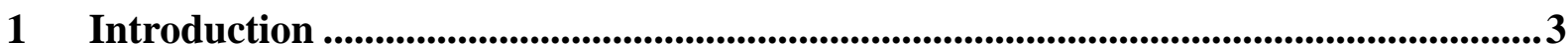

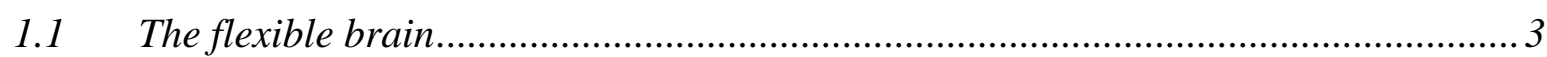

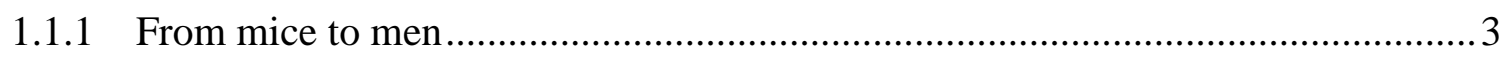

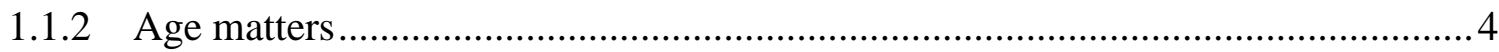

1.1.3 Plasticity mechanisms ...............................................................................

1.2 The visual system and ocular dominance plasticity ............................................. 13

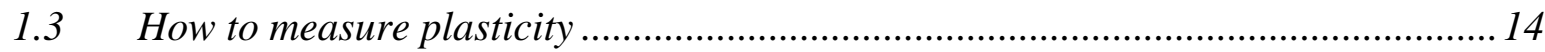

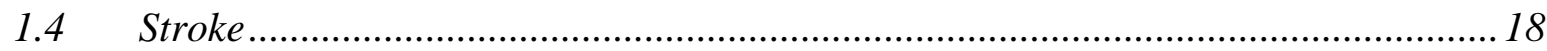

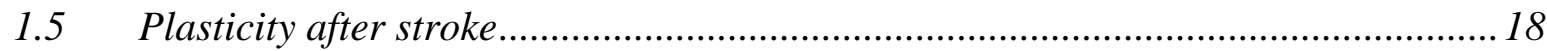

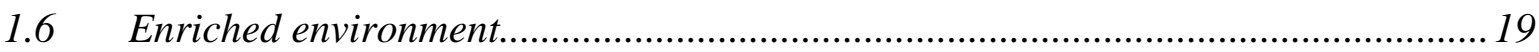

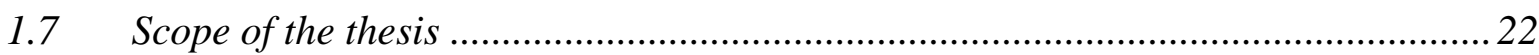

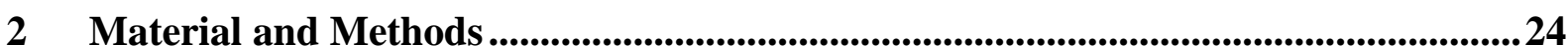

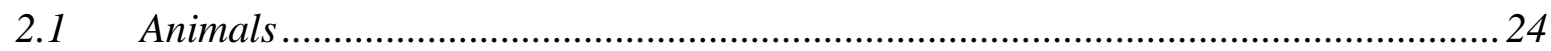

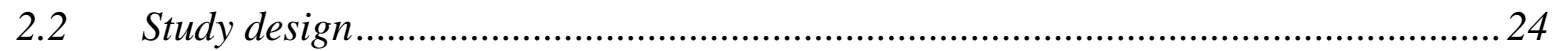

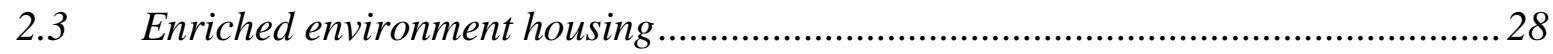

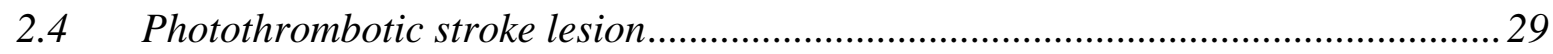

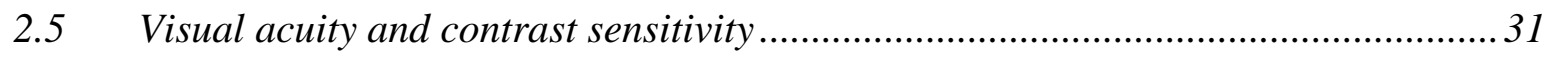

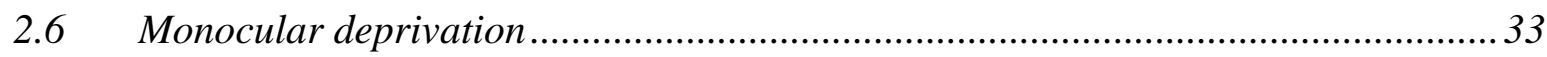

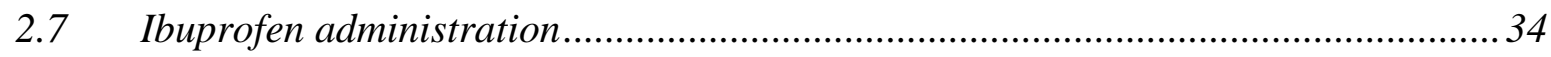

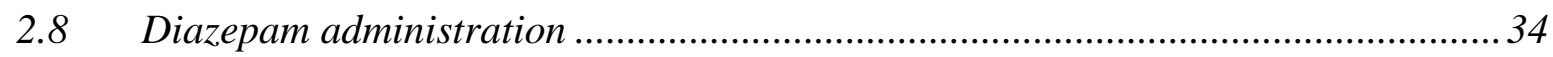

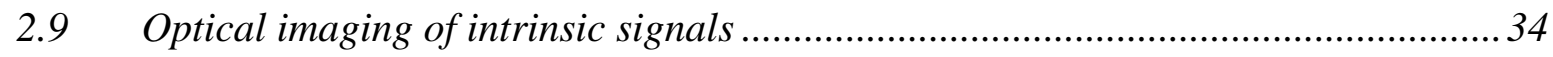

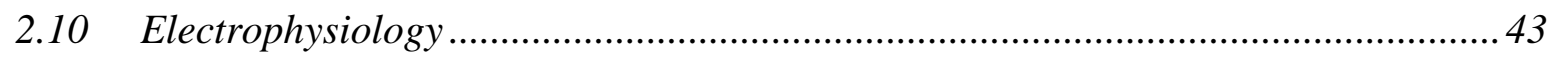

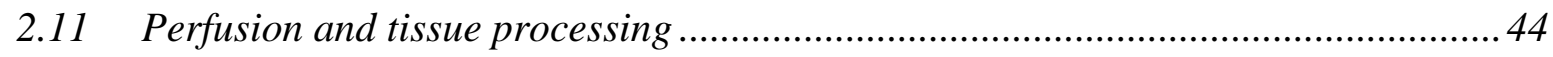

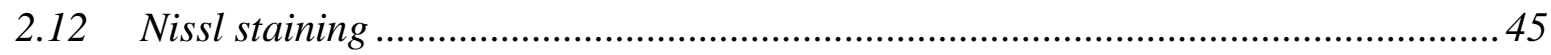




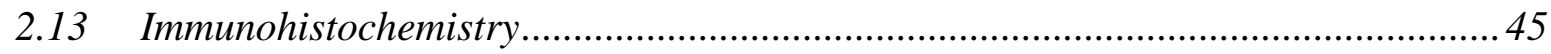

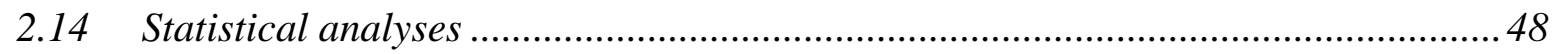

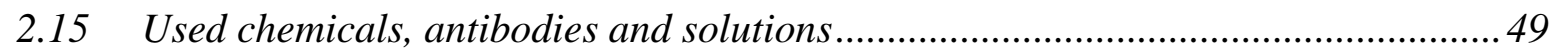

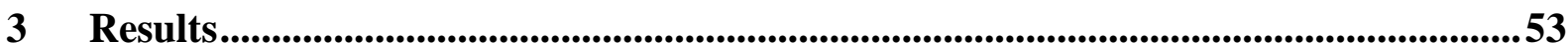

3.1 Impairment of visual plasticity after a cortical stroke .........................................5 53

3.1.1 Localization and size of the photothrombotic lesion........................................53

3.1.2 Photothrombosis abolished enhancement of vision after MD .............................56

3.1.3 OD-plasticity was impaired in the surround of a PT-lesion ................................61

3.1.4 OD-plasticity was impaired in the surround of a PT-lesion - electrophysiological results. 66

3.1.5 OD-plasticity after PT was still present in the non-lesioned hemisphere - distinct effects of MD in the non-lesioned and lesioned hemisphere..............................67

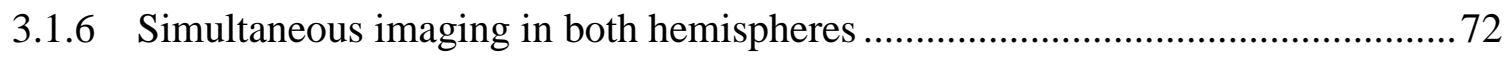

3.2 Therapeutic restoration of visual plasticity after a cortical stroke ........................ 75

3.2.1 Ibuprofen and late MD restored enhancement of vision after MD ...................... 75

3.2.2 Ibuprofen and late MD did not restore OD-plasticity ....................................... 87

3.3 Overview of OD-plasticity in both hemispheres................................................... 90

3.4 Environmental enrichment extended juvenile OD-plasticity into adulthood and restored it in old mice ................................................................................................... 93

3.4.1 EE extended juvenile OD-plasticity into adulthood ..........................................93

3.4.2 EE restored OD-plasticity in old mice............................................................. 98

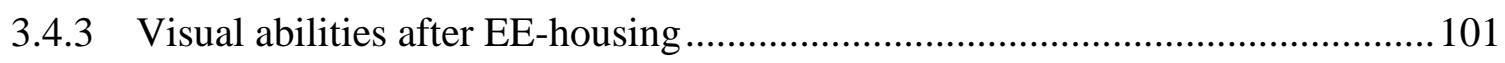

3.4.4 Diazepam treatment only partly abolished OD-plasticity …............................. 107

3.4.5 Diazepam treatment did not affect enhancement of visual acuity ...................... 111

3.4.6 EE-rearing did not affect the number of parvalbumin-positive cells and perineuronal nets.

3.5 Can enriched environment protect from stroke impairments? ............................. 117

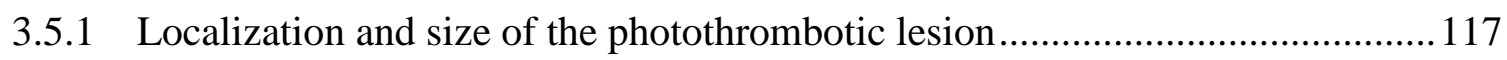

3.5.2 EE preserved OD-plasticity after a photothrombotic lesion............................. 118

3.5.3 EE partially preserved enhancement of vision after MD ............................... 122 


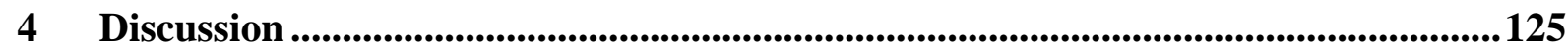

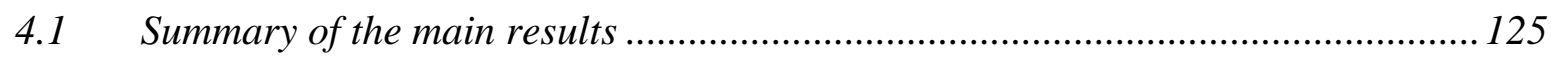

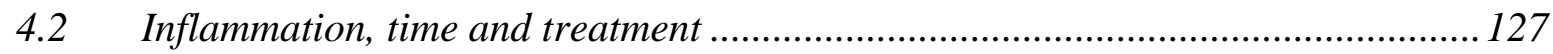

4.3 Reduced or increased plasticity?.............................................................. 130

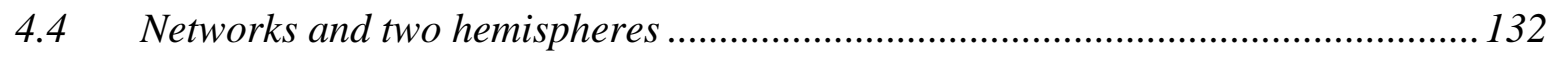

4.5 Enriched environment boosts OD-plasticity................................................... 139

4.6 Are inhibitory circuits modified after EE in mice?.......................................... 141

$4.7 \quad$ What other possible mechanisms are there? .................................................... 143

4.8 How can EE preserve OD-plasticity after stroke? ............................................ 146

4.9 Which EE-components are actually effective? .................................................. 148

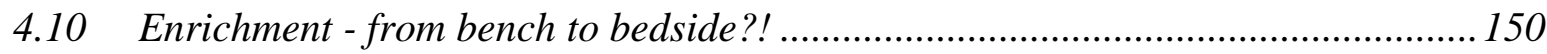

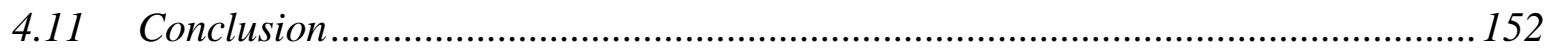

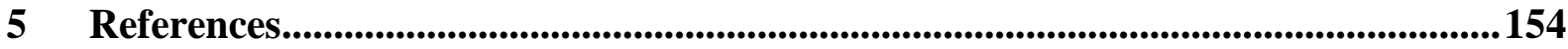

Abbreviations ................................................................................................................................. 172

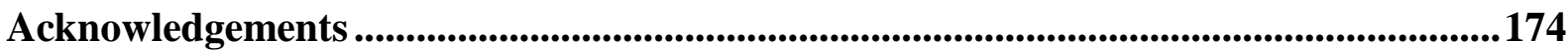

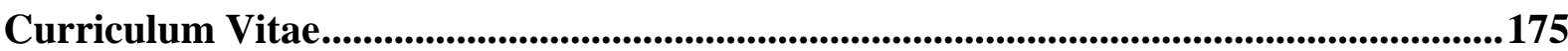




\section{ABSTRACT}

Stroke is a major cause of death and disability in industrialized countries. Cortical plasticity is crucial for recovery and rehabilitation after a stroke. The aims of the study were to investigate the impact of a photothrombotically induced stroke (PT) in the primary somatosensory cortex (S1) on sensory learning and plasticity of the neighboring primary visual cortex (V1) in adult C57BL/6J mice. To challenge plasticity mechanisms, we used monocular deprivation (MD), a well-established model for experience-dependent plasticity in the visual system. Sensory learning, i.e. the increase of visual acuity and contrast sensitivity of the open eye after MD, was analyzed with a behavioral test, a virtual reality system based on the optomotor reflex. In addition, visual cortex activity and maps were recorded using intrinsic signal optical imaging in the same mice. In sham-operated control animals, MD induced a significant increase of visual abilities of the open eye and a significant ocular dominance (OD) shift towards this eye. In contrast, in lesioned animals, there was neither an increase in visual abilities nor an OD-shift in the lesioned hemisphere. Since the PT-lesion was located outside V1, the ODplasticity in V1 must be affected by network influences. However, OD-plasticity was still present in the non-lesioned hemisphere. Since stroke is associated with inflammation, the therapeutic effect of the anti-inflammatory drug ibuprofen was tested: daily intraperitoneal injections of ibuprofen restored enhancement of vision but not OD-plasticity in the lesioned hemisphere. Consistent with this, a delay of 2 weeks between PT and MD also restored the enhancement of vision, but not OD-plasticity. Thus, inflammation was at least partly responsible for reductions in sensory learning in this paradigm, but lesion-induced impairment of OD-plasticity was mediated by a different cellular mechanism. We conclude that (i) both, sensory learning after PT and cortical plasticity in the surround of a cortical lesion are impaired; (ii) most likely a transient inflammation is responsible for impaired sensory learning, suggesting anti-inflammatory treatment with ibuprofen as a useful adjuvant therapy to support rehabilitation following stroke; and (iii) OD-plasticity cannot be just a local process and non-local influences are probably more important than previously assumed.

Moreover, we tested enriched environment (EE) housing as a non-invasive strategy to enhance plasticity in mice. OD-plasticity in mice is at its maximum at 4 weeks, declines in 2 to 3 months old animals and is absent beyond postnatal day (PD) 110 if mice are raised in standard cages (SC) (Lehmann \& Löwel 2008). Since EE has been shown to promote plasticity mechanisms in the adult rodent brain (e.g. Sale et al. (2007)), we tested whether raising mice in EE could prolong the sensitive phase for OD-plasticity. To this end, we 
housed mice from 7 days before birth into adulthood (> PD 110) in EE, an age at which ODplasticity is no longer present in SC-mice. Our results show that EE not only preserved ODplasticity but created adult mice with juvenile-like OD-plasticity, even up to PD 196. Administration of diazepam to increase inhibition significantly reduced but did not completely abolish the EE-induced preservation of OD-plasticity into adulthood, indicating that the plasticity enhancing effect of EE was at least partly mediated by a reduced inhibitory tone. Using immunofluorescence, we found that the number of parvalbumin (PV)-positive labeled cells and WFA-positive labeled perineuronal nets (PNNs) in V1 were not changed by EE-housing. Furthermore, EE restored already lost plasticity: when SC-mice were transferred to EE at PD 110 (late EE), OD-plasticity was restored, even up to PD 320. To test whether EE might be used to prevent compromised plasticity after a photothrombotically induced stroke we again raised mice in EE and then exposed them to a stroke. Indeed, in adult EE-mice, ODplasticity was present even after stroke and the improvement of visual abilities was partially preserved. Taken together, (i) EE preserved a juvenile-like OD-plasticity into late adulthood; (ii) which partially depended on inhibition. Moreover, EE-housing also (ii) restored ODplasticity in SC raised animals and (iii) raising mice in an EE preserved OD-plasticity even after a PT-stroke. 


\section{INTRODUCTION}

\subsection{The flexible brain}

The old assumption that connections in the adult brain are rigid and no longer modifiable is luckily not true. Indeed, the brain is capable to adapt to new demands the whole life long, which is essential for learning processes and to adapt to our environment as a result of active training, passive exposure or if we suffer from a brain injury (Merzenich et al. 1983; Clark et al. 1988; Nudo 2003b; a). That is what we call neuronal plasticity: the ability of the brain to structurally and functionally adapt in response to modified inputs from the environment. The ability for plasticity is especially high during a defined period in early life: the critical period.

The ocular dominance plasticity of V1 is a well-established model for neuronal plasticity. The binocular part of V1 gets input from both eyes, but neurons respond better to stimulation of one eye than to stimulation of the other eye. That is called "ocular dominance" (OD) and refers to the balance between responses to the each eye. In the 60ies, Hubel and Wiesel performed the first detailed investigation of a critical period at neuronal level in the visual cortex (Wiesel \& Hubel 1963b). Depriving young cats during the early postnatal development of normal binocular visual experience by closing one eye (MD) drastically altered the OD in their visual cortex. Electrophysiological recordings showed that the deprived eye stimulation became less effective in driving cortical cells, whereas the open eye gained influence. Thus, the MD induced an OD-shift (in other words OD-plasticity) towards the open eye (Wiesel \& Hubel 1963b). The OD-distribution had shifted so that nearly all cells were activated by stimulation of the eye that had remained open. This experimental paradigm of OD-plasticity is widely used to study experience-dependent cortical plasticity.

\subsubsection{From mice to men}

Extensive studies about OD-plasticity were not only done in cats (Hubel \& Wiesel 1962; Wiesel \& Hubel 1963b; a), but also in monkeys (Hubel et al. 1977), ferrets (Issa et al. 1999), rabbits (Van Sluyters \& Stewart 1974), hamsters (Emerson et al. 1982), rats (Maffei et al. 1992; Fagiolini et al. 1994) and mice (Dräger 1978; Gordon \& Stryker 1996; Bartoletti et al. 2002; Sawtell et al. 2003; Hofer et al. 2006b; Lehmann \& Löwel 2008; Greifzu et al. 2011; Ranson et al. 2012; for review see Espinosa \& Stryker 2012; Levelt \& Hübener 2012). Mice are extensively used to study OD-plasticity because visual cortex plasticity in mice is similar to cats, monkeys and presumably humans: it is age-dependent and expresses a critical period 
(Hubel \& Wiesel 1970; LeVay et al. 1980; Gordon \& Stryker 1996; Lehmann \& Löwel 2008). Since the visual system is one of the most studied parts of the mammalian central nervous system, we can take advantage of the existing knowledge and use the mouse as a basic system for studies in fundamental research. Another strong argument for the use of mice is the increasing availability of genetically modified mice, which allows us to gain completely new insights into cellular and molecular mechanisms underlying neuronal plasticity. Therefore, the visual system of mice is a suitable model to analyze the visual system, the plasticity of the brain and can be used as an animal model for neurological diseases, like stroke, in humans.

\subsection{2 $\underline{\text { Age matters }}$}

Juvenile mice - the critical period for OD-plasticity. During the critical period in juvenile mice, a prominent OD-shift in the visual cortex can be induced with just 4 days of MD (Gordon \& Stryker 1996; Frenkel \& Bear 2004; Hofer et al. 2006b; Lehmann \& Löwel 2008; Sato \& Stryker 2008). This critical period for easily inducible OD-plasticity occurs in mice between PD 19 and 32 (Gordon \& Stryker 1996). The OD-plasticity during the critical period in mice can be characterized by 3 consecutive stages (reviewed in Espinosa \& Stryker 2012). The first 2 to 3 days of MD result in a reduction of deprived eye responses after visual stimulation (Sato \& Stryker 2008). In the second stage, a longer MD-period of about 7 days results in an increase in the open eye responses and a further shift of OD (Kaneko et al. 2008b; Sato \& Stryker 2008). The third stage occurs if the deprived eye is reopened during the critical period and thereby binocular vision is restored: responses of both eyes rapidly return to their baseline level (Kaneko et al. 2008a). These stages were similar irrespective whether the contralateral or the ipsilateral eye was deprived (Sato \& Stryker 2008).

Adult mice. OD-plasticity in adult mice (PD 60-90) is different to that in the critical period in several aspects. After the critical period the OD-plasticity declines, but can still be induced in 2 to 3 months old mice with a longer deprivation period of 7 days (Sawtell et al. 2003; Lehmann \& Löwel 2008; Sato \& Stryker 2008). This OD-shift after 7 days MD is still smaller than after 4 days MD during the critical period. In adult mice the OD-shift is predominantly mediated by an increase of the open eye responses (Sawtell et al. 2003; Hofer et al. 2006b; Sato \& Stryker 2008). Moreover, deprivation of the ipsilateral eye in adult mice does not result in a significant OD-shift (Sato \& Stryker 2008). In mice older than 110 days of age, OD-plasticity, as measured by optical imaging of intrinsic signals, is absent and no OD-shift can be induced any more (Lehmann \& Löwel 2008), not even after 14 days of MD (Greifzu et 
al. 2012). The molecular and cellular changes which underlie OD-plasticity and how plasticity in the aging brain can be enhanced is investigated intensively (for recent reviews see Bavelier et al. 2010; Smith \& Bear 2010, Baroncelli et al. 2011). Mechanisms that underlie ODplasticity in the healthy brain are likely to be crucial for regeneration after brain lesions as well.

\subsubsection{Plasticity mechanisms}

There are several mechanisms that control and limit plasticity in the juvenile and adult brain (for recent reviews, see Bavelier et al. 2010; Espinosa \& Stryker 2012; Levelt \& Hübener 2012). They will be reviewed more in detail in this chapter.

The critical period as a phase of elevated plasticity, depends on a subtle balance of inhibition and excitation of neuronal circuits in the brain (reviewed in Chattopadhyaya et al. 2004). The development of inhibitory circuits in the cortex is crucial for the onset and time course of critical periods (reviewed in Hensch 2005). Cortical inhibitory interneurons account for $15 \%$ to $30 \%$ of all cortical neurons (reviewed in De Felipe 2002). The majority of cortical interneurons use GABA ( $\gamma$-aminobutyric acid) as neurotransmitter, which is an important inhibitory neurotransmitter in the mammalian central nervous system (for a review see McCormick 1992; Moore 1993). Two studies demonstrated that GABA is fundamental for the onset of the critical period of OD-plasticity in rodents. In the first study, Hensch et al. (1998) showed that the development of the GABAergic innervations and therefore a certain minimum level of inhibition is crucial for the onset of the critical period. When the GABAsynthesizing enzyme GAD65 (glutamic acid decarboxylase 65) was genetically inactivated (GAD65-knockout mice) and therefore GABA synthesis was reduced, the critical period of OD-plasticity was not initiated and no OD-plasticity was inducible. Treating these mice with the diazepam (positive allosteric $\mathrm{GABA}_{\mathrm{A}}$ receptor modulator) could reinduce OD-plasticity, demonstrating that a lack of inhibition abolished the critical period onset. However, one has to keep in mind that GAD65 knockout mice not only show altered inhibition, but also have alterations in their glutamatergic N-methyl-D-aspartate (NMDA) receptor subunit composition and function (Kanold et al. 2009), which might affect OD-plasticity. In the second study, Fagiolini and Hensch (2000) observed that treating young wild-type mice with diazepam can open a precocious critical period, indicating that early enhancement of GABAergic inhibition triggers the precocious onset of OD-plasticity. Subsequent studies showed that for the opening of the critical period via GABAergic transmission, especially $\mathrm{GABA}_{\mathrm{A}}$ receptors with the $\alpha 1$ subunit are crucial. Fagiolini et al. (2004) showed that $\mathrm{GABA}_{\mathrm{A}}$ 
receptors, which contain the $\alpha 1$-subunit, are required for the induction of critical period ODplasticity. The $\alpha 1$-subunit is enriched in inhibitory synapses formed by fast-spiking PVergic interneurons (Klausberger et al. 2002). Double labeling experiments with GABA-antibodies have shown that all PV-positive cells in the adult (> PD 90) mouse V1 are GABAergic (Gonchar et al. 2007). Within the GABAergic interneurons, PVergic neurons represented with $39 \%$ the largest group (Gonchar et al. 2007). There are at least 12 more distinct groups of GABAergic interneurons, like calretinin, somatostatin and neuropeptide Y expressing cells (Gonchar et al. 2007). Furthermore, site specific optimization of $\mathrm{GABA}_{\mathrm{A}}$ receptor numbers triggers the onset of OD-plasticity in the visual cortex (Katagiri et al. 2007). Hence, these findings suggest that PV-positive cells play an important role in opening the critical period plasticity.

As mentioned, the maturation of inhibitory circuits is crucial for the opening of the critical period. Factors that control the opening of the critical period often also regulate the maturation of inhibitory neurons in V1. Transgenic mice overexpressing the brain-derived neurotropic factor (BDNF) during postnatal development show a precocious critical period, probably because BDNF enhances the maturation of GABAergic inhibition (Hanover et al. 1999; Huang et al. 1999; Gianfranceschi et al. 2003). Likewise, other growth factors like IGF1 (insulin-like growth factor) can also accelerate the maturation of circuitry and inhibitory innervations (Tropea et al. 2006; Ciucci et al. 2007) (reviewed in Lodovichi et al. 2000). Another study suggest that also polysialic acid (PSA), which is attached to the neural cell adhesion molecule (NCAM) is involved in the opening of the critical period for OD-plasticity and the maturation of inhibitory circuits (Di Cristo et al. 2007).

In contrast to GABA, which is an inhibitory neurotransmitter in the adult brain, glutamate is an excitatory neurotransmitter (for a review see McCormick 1992; Moore 1993). The NMDA receptor is an ionotropic glutamate receptor that is essentially involved in plasticity in the cortex. NMDA receptor signaling is important for OD-plasticity in adult mice: if NMDA receptor activity is blocked by injection of the competitive NMDA receptor antagonist CPP (3-(2-carboxypiperazin-4-yl)-propyl-1-phosphonic acid) during MD (Sato \& Stryker 2008) or if the adult mice lack the NMDA receptor subunit NR1 (Sawtell et al. 2003), OD-plasticity is diminished. Moreover, calcium influx through NMDA receptors regulates protein kinases and phosphatases that affect OD-plasticity, like the cAMP-dependent protein kinase (PKA) subunits RII- $\alpha$ and RII- $\beta$ (Fischer et al. 2004; Rao et al. 2004), extracellular-signal-regulated kinase (ERK) (Di Cristo et al. 2001), the $\alpha$-calcium/calmodulin-dependent protein kinase II ( $\alpha$ CaMKII) (Taha et al. 2002), and the phosphatase calcineurin (Yang et al. 2005). In these 
studies, reducing the activation of the kinases or increasing the activation of the phosphatase reduced OD-plasticity in the critical period. In adult mice, which have a point mutation that prevents autophosphorylation of $\alpha \mathrm{CaMKII}$ ( $\alpha \mathrm{CaMKII}{ }^{\mathrm{T} 286 \mathrm{~A}}$ mice), OD-plasticity was also absent (Ranson et al. 2012). Nuclear factors that influence visual cortex plasticity are the activity-dependent immediate early gene Arc (activity-regulated cytoskeleton-associated protein) (Tagawa et al. 2005; McCurry et al. 2010) and CREB (cAMP response elementbinding protein), which mediates gene transcription (Pham et al. 1999; Mower et al. 2002; Putignano et al. 2007). In the developing visual mouse cortex, tumor necrosis factor- $\alpha$ (TNF$\alpha$ ), an important protein for homeostatic scaling of excitatory and inhibitory synapses (Stellwagen \& Malenka 2006), is also involved in OD-plasticity. TNF- $\alpha$ knockout mice showed the normal initial loss of deprived-eye responses after MD, but no subsequent increase in response to the open eye (Kaneko et al. 2008b). Nevertheless, adult OD-plasticity appeared to be unaffected in TNF- $\alpha$ knockout mice (Ranson et al. 2012).

In order to reveal anatomical changes after MD, Hubel et al. (1977) did tracing experiments in monkeys in which they injected a radioactive tracer into one eye. This tracer was transported transneuronally via the lateral geniculate nucleus (LGN) into the thalamus to the cortical terminals in V1. After MD in young animals, they observed a contraction of thalamocortical projections from the deprived eye and an expansion of the projections from the open eye. These structural changes in thalamocortical projections have also been found in cats and mice (Shatz \& Stryker 1978; Antonini \& Stryker 1996; Antonini et al. 1999). Another structural effect of MD in the visual cortex during early life is the alteration of motility, turnover, number, and morphology of dendritic spines, which receive mainly input from excitatory synapses (Majewska \& Sur 2003; Mataga et al. 2004; Hofer et al. 2009). Dendritic spines are considered as crucial during the reductions in deprived eye responses after MD during the critical period. However the mechanism is not quite clear yet. The decrease in closed eye responses might be due to anatomical pruning, like loss of synapses/spines driven from the deprived eye, or reduction in synaptic efficacy by LTD (long-term depression)-like mechanism (including removal of ionotrophic receptors), within stable anatomical circuits or a combination of both mechanisms (reviewed in Espinosa \& Stryker 2012). In adult mice, increased spine dynamics seem also to contribute to MD effects (Hofer et al. 2009), but other plasticity mechanisms may be more important in adult V1, like changes in inhibitory input (Maffei et al. 2006; Kameyama et al. 2010; van Versendaal et al. 2012). 


\section{"Brakes" of OD-plasticity}

After the critical period OD-plasticity declines and remains restricted during adulthood. There are several cellular and molecular "brakes" that close the critical period and keep it closed later on (for recent reviews, see Bavelier et al. 2010; Espinosa \& Stryker 2012; Levelt \& Hübener 2012). Some of these "brakes" are physiological and act on the excitatory-inhibitory balance, some are structural (Figure 1). Several mechanisms and the potential capacity to enhance plasticity in adulthood will be reviewed here.

(1)

\section{Removing structural brakes}

(2)

\section{Re-setting E-I balance - feedback signaling}

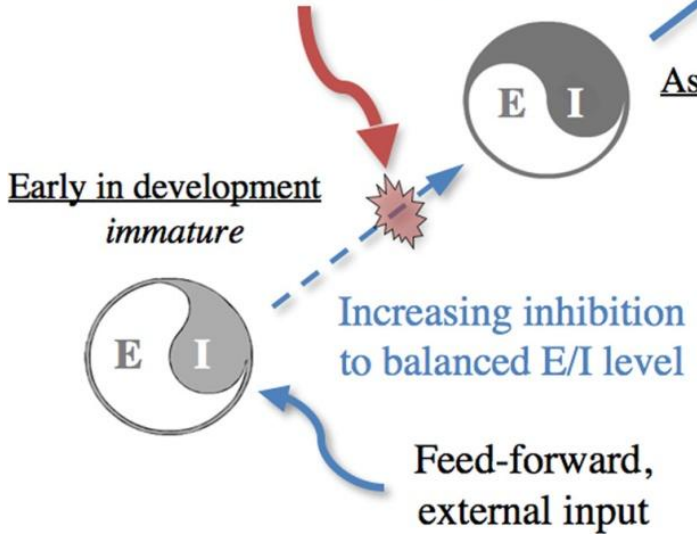

Adult

consolidated

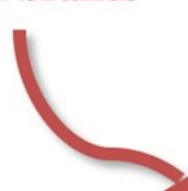

3

Structural changes that

limit new connectivity

\section{As development proceeds plastic}

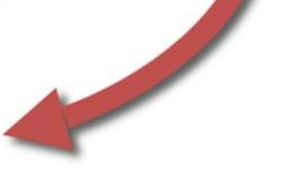

\section{Intervention pushes \\ the system into a plastic state}

Figure 1: The change of plasticity capacity across lifespan (blue arrows) and possible mechanisms that enhance plasticity and recovery of function (red). Early in development (before the critical period) the excitatory-inhibitory ratio (E/I) is in an immature state and an increase of the inhibition is necessary to gain a balanced E/I level during the critical period, and therefore allow plastic changes. With age, structural changes and increased inhibition limit plasticity. (1) By removing structural barriers, like perineuronal nets, brain plasticity can be restored. (2) Resetting the E/I balance to a juvenile state can also promote plasticity in adulthood. (from Bavelier et al. (2010))

Once the critical period is opened, the GABAergic inhibitory system in the visual cortex matures and correlates with the closure of the critical period for OD-plasticity (Huang et al. 1999; Morales et al. 2002; Chattopadhyaya et al. 2004). In accordance with that, a reduction of GABAergic transmission in the visual cortex of adult rats by infusion of picrotoxin (PTX, reduces GABAergic transmission by antagonizing $\mathrm{GABA}$ action on its $\mathrm{GABA}_{\mathrm{A}}$ receptors) or 
3-mercaptopropionic acid (MPA, inhibitor of the activity of GABA synthetic enzyme GAD) into V1 can partially reactivate OD-plasticity (Harauzov et al. 2010) (Figure 1).

Structural factors. Structural factors can also limit OD-plasticity. One of them is the extracellular matrix. The extracellular matrix consists of a complex network of molecules that fills the space between the cells for structural support and other important functions (for a review see Dityatev et al. 2006). One major component of the extracellular matrix are the chrondroitin-sulfate proteoglycans (CSPGs) (reviewed in Galtrey \& Fawcett 2007). During development, CSPGs condense into lattice-like structures, known as PNNs, around neurons. CSPGs structurally and functionally limit adult plasticity (reviewed in Berardi et al. 2004). When CSPGs are degraded by the enzyme chondroitinase ABC, OD-plasticity could be reactivated and recovery from long-term $\mathrm{MD}$, in the sense of restored visual acuity and binocularity of cortical neurons, could be promoted in adult rats (Pizzorusso et al. 2002; Pizzorusso et al. 2006) (Figure 1). This suggests that the adult extracellular matrix is an important factor limiting structural plasticity. Moreover, the formation of PNNs in the visual system also correlates with the end of the critical period for OD-plasticity (Pizzorusso et al. 2002; for reviews see Berardi et al. 2004; Hensch 2005). The formation of PNNs is triggered by neuronal production of the cartilage link protein 1 (Crtl1). It is upregulated during development in the visual cortex while PNNs form (Carulli et al. 2010). Mice lacking Crtl1 have diminished PNNs and retain juvenile levels of OD-plasticity during adulthood (Carulli et al. 2010). Matrix metalloproteinases (MMPs) are involved in the degradation of the extracellular matrix (reviewed in Huntley 2012). A recent in vivo study demonstrated the role of MMPs in regulating OD-plasticity in juvenile rats (Spolidoro et al. 2012). Using electrophysiology measurements, they showed that a MMP inhibitor prevented the increase in open eye responses after MD and thereby impaired OD-plasticity. Inhibitory PVergic neurons, which get enwrapped by PNNs, are important for the onset and offset of the critical period (Huang et al. 1999; Fagiolini et al. 2004), and, as mentioned, a critical level of intracortical inhibitory tone is also required for experience-dependent plasticity during the critical period (Mataga et al. 2004). Hence, at least some of the effects of PNN degradation could be mediated by modifications of intracortical inhibitory circuits, which brings them back to a more juvenile-like state (Hensch 2005). The pathway via Otx2 is also involved in that PNNsPV connection. This transcription factor is transcribed and translated in the retina, and transported to the visual cortex where it accumulates within PVergic-cells by the peak of the critical period (Sugiyama et al. 2008). Otx2 accumulation promotes maturation of the PV-cell network and hence critical period activation in visual cortex. Otx 2 appears to enter PVergic 
interneurons through interactions with the PNNs that enwrap them (reviewed in Sugiyama et al. 2009). Knockout of Otx 2 prevented the maturation of PV-cells and the opening of the critical period. This effect could be counteracted by infusion of Otx2 into V1 (Sugiyama et al. 2008). Nevertheless, the impact of the extracellular matrix on cortical plasticity could also occur at the level of excitatory neurons, since PNNs, in a less pronounced way, can be also found around pyramidal neurons (Wegner et al. 2003; Alpar et al. 2006). Another explanation for limited plasticity due to the extracellular matrix might be that the mature extracellular matrix creates an environment that represses synaptic dynamics. Consistently, different studies showed a developmental decline of spine motility and pruning after visual deprivation in V1 (Majewska \& Sur 2003; Mataga et al. 2004; Oray et al. 2004). Furthermore, it has been observed that digestion of CSPGs caused a recovery of dendritic-spine density in the visual cortex of adult rats (Pizzorusso et al. 2006). Moreover, a protein that degrades the extracellular matrix, called tissue plasminogen activator (tPA), is increased during MD in mice and essential for OD-plasticity (Mataga et al. 2002). Consequently, this leads to the conclusion that extracellular matrix degradation facilitates structural remodeling of synaptic contacts between neurons and therefore plasticity.

Other structural factors that can limit OD-plasticity are myelin-associated proteins, like Nogo, the myelin-associated glycoprotein (MAG) and oligodendrocyte myelin glycoprotein (OMGP), that inhibit neurite outgrowth in the central nervous system (for review see Schwab 2010). Mice mutants lacking the receptors for these growth inhibitors, such as the paired immunoglobulin-like receptor B (PirB) (Syken et al. 2006; Atwal et al. 2008), Nogo-66 receptor $(\mathrm{NgR})$ or lacking Nogo-A/B itself (McGee et al. 2005), showed enhanced levels of OD-plasticity in adulthood that were comparable to those in immature mice.

Histone acetylation. The acetylation status of histones regulates the transcription of genes and therefore gene expression. Such epigenetic modification has been recently implicated in the regulation of plasticity in the adult visual cortex. Visual experience activates histone acetylation in the visual cortex during the critical period, but this capacity is downregulated in adult animals (Putignano et al. 2007). Histone deacetylases (HDACs) are enzymes that alter the chromatin structure by removing acetyl groups from histone core proteins and thereby causing a tighter wrapping of DNA around the histones (for a review see Fagioloni et al. 2009). This compaction of chromatin leads to transcriptional suppression. Pharmacological treatment with inhibitors of histone deacetylases consequently promotes histone acetylation. Treatment with an HDAC-inhibitor (trichostatin) enhanced OD-plasticity in adult mice: in 
contrast to control mice, 3 days of MD were enough to change OD (Putignano et al. 2007). Treatment with other histone deacetylases inhibitors (valproic acid and or sodium butyrate,) promoted recovery of visual acuity in adult rats after long-term MD (Silingardi et al. 2010). Consequently, epigenetic regulation of gene transcription is also associated with plasticity of the visual system.

\section{Influence of long-ranging interactions}

Neuromodulatory systems. The effects of the neuromodulatory systems are long-ranging, because the cell bodies of neuromodulatory neurons are grouped in specific nuclei in the forebrain (nucleus basalis: cholinergic system; Bruel-Jungermann et al. (2011)) and midbrain (locus coeruleus: noradrenergic; Sara (2009), raphe nuclei: serotonergic; Lesch \& Waider (2012), from where their widespread projections influence many brain regions, including the visual cortex. Bear and Singer (1986) modulated cholinergic and noradrenergic transmission in young cats by disrupting their innervations or changing it with drugs. They showed that a combination of both cholinergic and noradrenergic transmission was necessary to induce ODplasticity during the critical period. Later an analogous function was described for the serotoninergic (5-hydroxytryptamin, 5-HT) neuromodulatory system (Gu \& Singer 1995). A role for the cholinergic system in OD-plasticity of mice was also shown. The protein Lynx1 binds to acetylcholine receptors and reduces their sensitivity to acetylcholine. Lynx 1 expression in V1 of mice was increased only after the critical period for OD-plasticity (Morishita et al. 2010). When Lynx1 is knocked out, cholinergic transmission is enhanced and OD-plasticity is increased in adult mice: a short MD of 4 days was enough to induce ODplasticity in PD 60 mice (Morishita et al. 2010). This enhancement of OD-plasticity was abolished by infusion of nicotinic acetylcholine receptor antagonists or diazepam into V1. Treatment with the antidepressant drug fluoxetine, a selective serotonin reuptake inhibitor (SSRI), which increases serotonin the level, also increased OD-plasticity in adult rats (Maya Vetencourt et al. 2008). Likewise, this effect was abolished by diazepam infusions into V1. Taken together, these studies demonstrate that reduced neuromodulatory drive may restrain adult OD-plasticity, perhaps by perturbing inhibitory function. Moreover, the enhancement of neuromodulatory systems facilitated the induction of long-term potentiation (LTP) and LTD in in vitro studies (Brocher et al. 1992; Kojic et al. 1997; Kirkwood et al. 1999).

Cross-modal influences. Although sensory areas in the mammalian cortex, like the visual, motor and somatosensory area, respond predominantly to their according sensory modalities, anatomical projections link primary sensory areas, so that they also get input and get activated 
from other sensory regions (cross-modal influences), in humans as well as in rodents and primates (Paperna \& Malach 1991; Falchier et al. 2002; Rockland \& Ojima 2003; Merabet et al. 2007; Lim et al. 2012). In humans, who were blind from an early age on, V1 could be activated by somatosensory input like by Braille reading and other tactile discrimination tasks (Sadato et al. 1996; Cohen et al. 1997; Kupers et al. 2007). Moreover, tactile performance in blind and normally sighted, blindfolded subjects activated V1 (Merabet et al. 2007). Interestingly, transcranial electrical stimulation of the visual cortex of blind people during Braille reading impaired tactile perceptions and also Braille reading (Cohen et al. 1997). A recent study indicated that visual areas contribute also to olfactory processing in congenital blind (Kupers et al. 2011). Moreover, visual perception can be influenced by auditory stimuli. For example, subjects rated the intensity of a light higher when it was accompanied by a brief auditory stimulus, than when it was presented alone (Stein et al. 1996; review: Shams \& Kim 2010). Such cross-modal influences on the visual cortex are also known in rodents. Responses of V1 neurons (electrophysiological recordings) were reported when rats received somatosensory stimulation because they used their whiskers to explore objects in complete darkness (Vasconcelos et al. 2011). When rats were freely exploring novel objects in the dark, firing rates in S1 as well as in V1 changed (Vasconcelos et al. 2011). To investigate whether these responses represent behaviorally relevant information, Vasconcelos et al. (2011) carried out an experiment in which rats had to perform a whisker-related discrimination task in the dark. When animals had to discriminate between narrow and wide apertures using their whiskers in the dark, the tactile input triggered V1 spike responses. Indeed, the stimulusrelated responses in $\mathrm{V} 1$ correlated significantly with the success rate in the discrimination task. A recent calcium 2-photon imaging study showed strong motor-related input in V1 of awake mice in addition to visual input (Keller et al. 2012). Using electrophysiological recordings in the visual cortex of rats, Wallace et al. (2004) observed that individual neurons get also activated by auditory and somatosensory stimuli. Moreover, single whisker stimulation in rats evoked cortical activation that spreads far beyond S1 into multiple cortical territories of different modalities, including the visual, auditory and motor cortex (Frostig et al. 2008). These results clearly show that V1 gets functional input from several other cortical regions.

Interhemispheric interactions. Long-ranging interactions are also mediated through the corpus callosum which connects the two hemispheres. Callosal connections exist also between the visual cortices and influence experience-dependent plasticity (reviewed in Pietrasanta 2012). In rats, a substantial portion of the influence of the ipsilateral eye on cortical responses arrived 
via callosal connections from the opposite hemisphere, and these afferents played an important role in the reduction of inputs from the deprived eye during MD (Restani et al. 2009). These long-ranging intercortical interactions also mediated the intercortical inhibition: both hemispheres inhibit each other through the corpus callosum (Hummel \& Cohen 2006).

\subsection{The visual system and ocular dominance plasticity}

For a deeper understanding, the visual system and OD-plasticity will be described more in detail in this chapter.

The mouse visual system is similar to the human visual system in many ways. Information from visual stimuli are first transduced and processed in the multilayered retina and then transmitted as action potentials in the optic nerve. On the way to the visual cortex, a part of the optic nerves of both eyes cross in the optic chiasm (Figure 2).

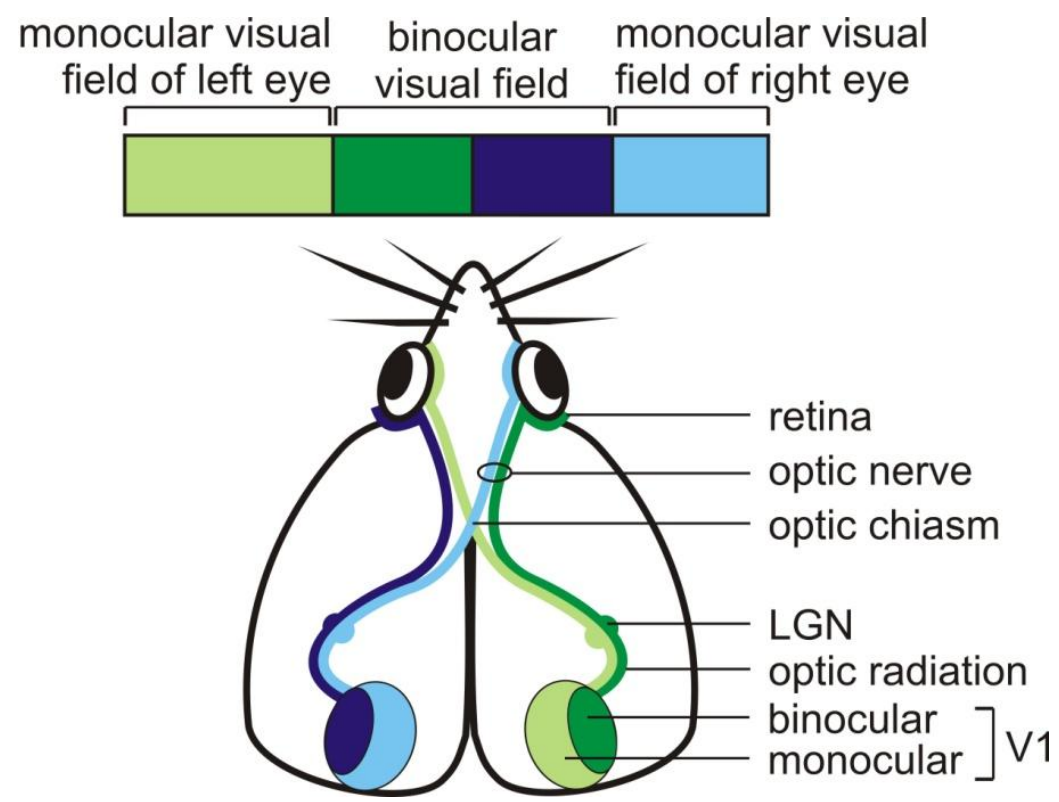

Figure 2: Visual pathways of a C57BL/6J mouse. The right (blue) and left (green) visual field and their representation in V1 are illustrated. Projections from the nasal part of the retina cross to the other hemisphere in the optic chiasm, whereas fibers from the temporal part of the retina do not cross. Therefore, the right visual field is represented in the left hemisphere and vice versa. While the binocular part of $\mathrm{V} 1$ receives input from both eyes, the monocular part of V1 receives just input from the contralateral eye. LGN = lateral geniculate nucleus. Modified from Greifzu et al. (2012). 
Nerve fibers from the medial (nasal) part of the retina cross to the other hemisphere in the optic chiasm, whereas nerve fibers from the lateral (temporal) part of the retina do not cross. From there the fiber tract is called optic tract and proceeds to the lateral geniculate nucleus in the thalamus, from where optic radiation leads to V1 (Figure 2). As a result of the nerve crossing at the optic chiasm nerve fibers from the left part of the retina of both eyes project to the left hemisphere and vice versa. Since the right visual field activates the left part of the retinae, information will arrive at the left hemisphere and information from the left visual field arrive via the right part of the retinae in the right visual cortex (Figure 2). Spatial neighborhood relations are preserved all along the visual, i.e., adjacent stimuli in the visual field activate adjacent neurons in V1. Thus, the visual field is represented topographically in the visual cortex as a retinotopic map.

V1 is divided into a monocular and a binocular part (Figure 2). Monocular nerve cells are exclusively activated by stimulation of the contralateral eye, whereas binocular cells can be activated by visual stimulation of both eyes. The binocular zone is represented in the lateral part of V1 and takes up about one third of it (Figure 2). The central part of the visual field (about 30-40 of each hemifield) (Gordon \& Stryker 1996), is represented in the retina of both eyes and therefore in the binocular zone. In mice, nerve cells in the binocular visual cortex are typically dominated by contralateral input: they show a stronger response after stimulation of the contralateral eye and a weaker one after ipsilateral eye stimulation (Dräger 1975; Mangini \& Pearlman 1980; Wagor et al. 1980; Metin et al. 1988). This is referred to as contralateral dominance. The ratio of the cortical activity induced by contra- or ipsilateral eye stimulation can be used to calculate the ocular dominance index (ODI). Closing the "strong" contralateral eye of a mouse (MD) causes neurons in the contralateral binocular zone to get activated almost equally strongly by stimulation of each eye (Dräger 1975; Gordon \& Stryker 1996). Thus, their OD shifted towards the open eye. This is known as OD-plasticity and is one of the most established and studied models of neuronal plasticity.

\subsection{How to measure plasticity}

For a long time the classical method to study OD-plasticity in vivo in mice was the single cell electrophysiology to record action potentials from single nerve cells in the binocular zone of V1 (Dräger 1978; Gordon \& Stryker 1996; Fagiolini et al. 2004). Although a high spatial resolution can be achieved with this method, time-consuming and technically challenging experiments are necessary. A fast method to visualize and register visual cortex activity with 
an excellent signal-to-noise ratio is the minimally invasive optical imaging of intrinsic signals (Blasdel \& Salama 1986; Grinvald et al. 1986; Kalatsky \& Stryker 2003), which has recently been optimized for the analysis of OD-plasticity (Cang et al. 2005). This in vivo technique was used in our study. In contrast to the electrophysiological methods, where single neurons or groups of neurons are observed (Dräger 1978; Gordon \& Stryker 1996; Porciatti et al. 1999; Fagiolini et al. 2004), with optical imaging of intrinsic signals a larger area, covering networks of neurons, is analyzed. This method relies mainly on a change in reflection due to metabolic activity in the activated cortical area. Active brain regions can be visualized as dark areas, even through the intact skull, using a highly light-sensitive camera (Figure 3).
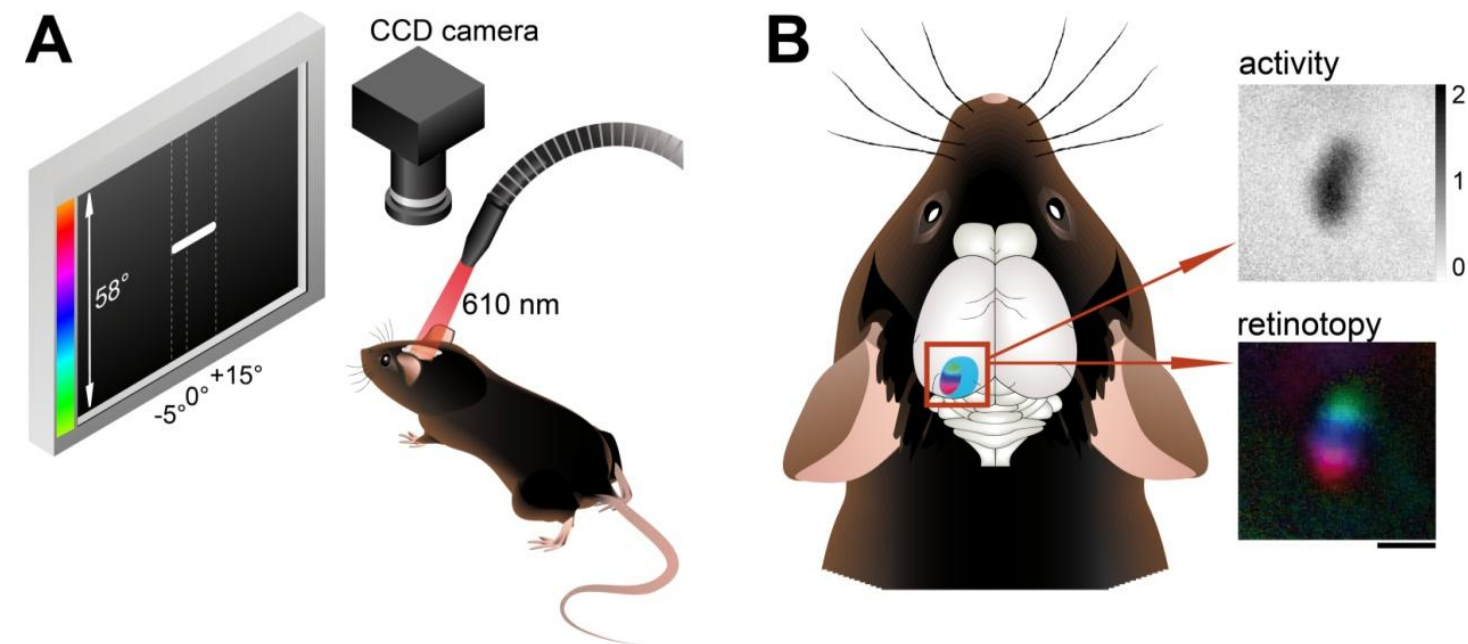

Figure 3: Visualization of neuronal activity. Neuronal activity in the visual cortex is visualized by the minimally invasive optical imaging of intrinsic signals. (A) The brain of the mouse is illuminated with red light $(610 \mathrm{~nm})$, a wavelength at which the change in reflection, due to activation of neurons, is reliably detectable. A visual stimulus is presented to the anesthetized mouse: a white horizontal bar on black background moving upwards or downwards on a monitor. The stimulus activates the binocular zone of V1. The activity changes in V1 are recorded using a light-sensitive CCD camera and are extracted by Fourier analysis. (B) An activity map (top) and retinotopic polar map (bottom) of the binocular zone of V1. The color-coded retinotopic map represents the neuronal response depending on the spatial position of the stimulus in the visual field. Scale bar: $1 \mathrm{~mm}$. Modified from Greifzu et al. (2012).

The signals which correlate with neuronal activity are intrinsic, meaning that no dyes are needed. With this technique neuronal activity and retinotopic maps can be visualized in the living animals within just 5 minutes, with a high spatial and temporal resolution (Bonhoeffer \& Grinvald 1996). The stimulus-induced signal can be clearly extracted from the heart, respiration, and vasomotor artifacts by Fourier analysis (Bonhoeffer \& Grinvald 1996; 
Kalatsky \& Stryker 2003). Optical imaging of intrinsic signals can be used to compare experience-, learning-, and age-dependent changes in brain activity in experimental animal groups with a different treatment. However, it is also possible to repeatedly image a single animal and therefore follow activity changes in one and the same animal over the course of time, for example before and after MD or drug treatment (Hofer et al. 2006b; Kaneko et al. 2008b; Greifzu et al. 2012). These long term chronic experiments offer the advantage of having an intra-animal control, i.e. measuring the same mouse before treatment (as control) and after treamtent.

In order to assess plasticity processes more comprehensively and to test whether the measured changes can also be found on the behavioral level, a visual behavioral test was performed additionally. The optomotor system developed by Prusky (2004) allows a fast and easy measurement of visual acuity and contrast thresholds (later converted into contrast sensitivity) in mice. The optomotor apparatus consists of 4 inward facing flat screen monitors that create an arena in which the mouse is positioned freely moving on an elevated platform (Figure 4).

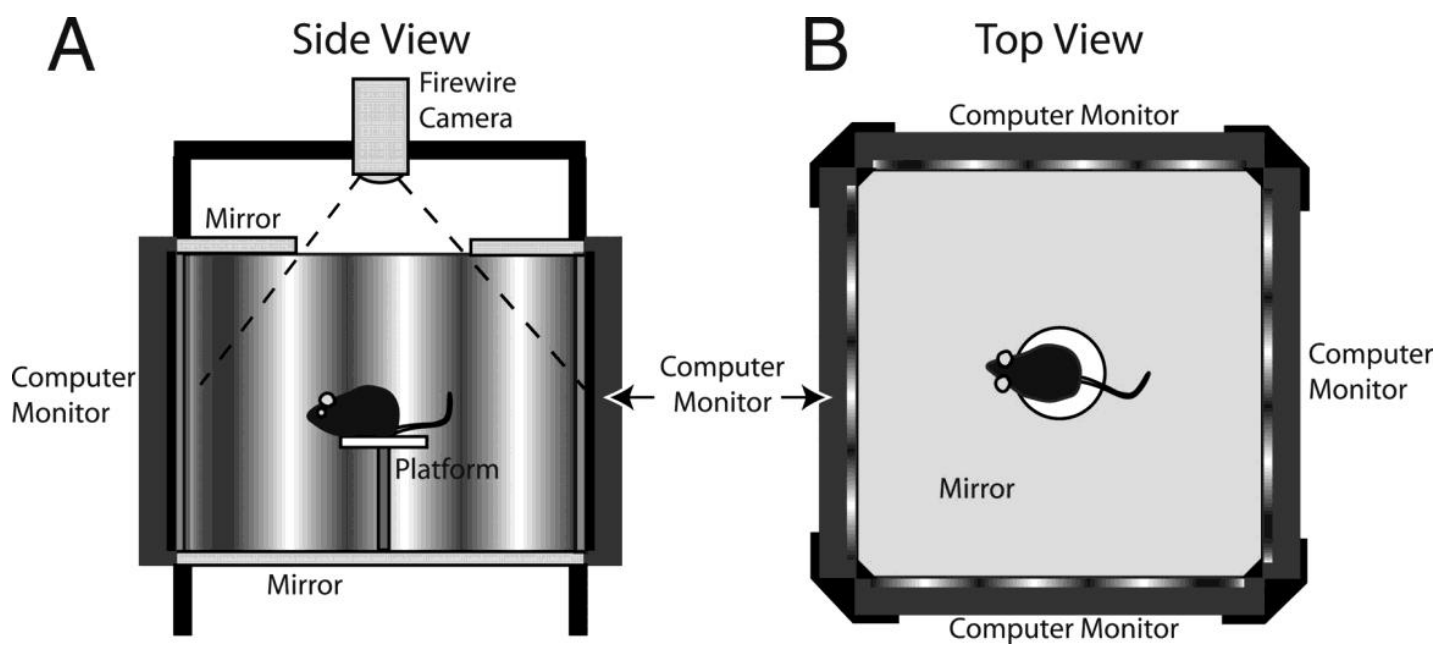

Figure 4: Scheme of the optomotor system. (A) Side view. A mouse is positioned on the platform in the middle of the testing arena, surrounded by computer monitors, on which a sine wave grating is projected. As long as the mouse can see the moving vertical gratings, it will follow them with reflexive head and body movements, which can be observed by an experimenter via a camera in the lid of the apparatus. (B) Top view. The mouse is surrounded by the grating and can move freely on the platform (Prusky et al. (2004)).

A virtual rotating cylinder with a vertical sine wave grating is projected on the computer monitors around the mouse. As long as the mouse can see the moving vertical gratings, it will follow them with reflexive head and body movements. 
It should be mentioned here that the "visual acuity" measured in the optomotor system does not correspond to the animals' maximal visual acuity. Nonetheless, the measured values are in a relatively constant relationship with maximal visual acuity, which can be measured for example with visual evoked potentials, optical imaging, or the visual water task (Porciatti et al. 1999; Prusky \& Douglas 2003; Goetze et al. 2010). Thus, the measurements with the optomotor system can be taken as a valid measure of the visual ability of mice.

The method of this optomotor system offers several advantages. First of all the measured reaction is based on a reflex - the optomotor reflex in response to a moving stimulus- and independent of the individual behavior of the mouse. In contrast to behavioral tests that are based on positive reinforcement and need extensive training, such as the visual water task (Gianfranceschi et al. 1999; Prusky et al. 2000b; Prusky \& Douglas 2003), the optomotor test does not need any training because it is based on a reflex. Thus, the visual abilities can be measured immediately at any time, also in very young mice and directly after MD. Visual acuity and contrast sensitivity of a mouse can be measured within just 15 minutes. While mechanically operated, striped cylinders (rotating drums) have been in use for a long time (Cowey \& Franzini 1979); the specific characteristic of the apparatus developed by Prusky et al. (2004) is the virtual cylinder. The distance of the presented grating and therefore its spatial frequency can always be adjusted precisely, even if the mouse is moving. Furthermore, the speed of the moving grating can be kept constant. Moreover, the measurements can be done for both eyes individually (Douglas et al. 2005). With this measurement of visual abilities, the plasticity induced by MD can be observed as an increase of visual acuity and contrast sensitivity of the open eye (Prusky et al. 2006). This plasticity also shows characteristics of classic learning paradigms, and is therefore called "sensory learning". The visual ability is increasing during testing sessions over the days due to the visual experience, it is declining again after reopening the MD ("forgetting") and is improving faster after another MD ("remembering") (Prusky et al. 2006).

In consequence, by combining optical imaging of intrinsic signals and the virtual reality optomotor system, it is possible to examine two models of experience- and learningdependent plasticity in the same animal: OD-plasticity as an established model of cortical plasticity and additionally the enhancement of visual acuity and contrast sensitivity of the open eye after MD as a model of sensory learning in a behavioral test. As cortical plasticity is crucial for recovery and rehabilitation after stroke, we used the combination of these two techniques to investigate the impact of a cortical stroke on plasticity and treatment possibilities for restoration. 


\subsection{Stroke}

Stroke is a sudden disruption of the blood supply to the brain due to a blocked or ruptured blood vessel. Thus, oxygen and nutrients can no longer be delivered. Nerve cells in the affected brain area die, what further may cause abrupt paralysis, disturbances of consciousness, language and perception (Bundesministerium für Bildung und Forschung (BMBF) 2003). Stroke is one of the leading causes of death in the industrialized countries. Even if the death rate could be reduced due to better examination and faster treatment, treatment strategies are still limited. Moreover, the majority of the patients suffer from persistent neurological damage (Bundesministerium für Bildung und Forschung (BMBF) 2003). In fact, stroke is one of the main reasons for the need of nursing care in adulthood (Robert Koch Institut 2006). Moreover, the incidence of a stroke increases with age (Feigin et al. 2003) and as people are becoming increasingly older, there will be also a growing number of stroke patients. Hence, effective strategies for stroke treatment and rehabilitation are essential.

\subsection{Plasticity after stroke}

Stroke not only destroys directly affected brain areas but also disturbs surrounding areas in the lesioned and even in the contralateral hemisphere (Buchkremer-Ratzmann et al. 1996; review: Andrews 1991). Recovery after stroke requires cortical plasticity: the rewiring of the brain by modulating the strength of synaptic connections and neuronal properties (Seitz et al. 2004). Several studies indicate that brain areas around an ischemic lesion show an increased plasticity: GABAergic inhibition is decreased (Neumann-Haefelin et al. 1995; Schiene et al. 1996; Qü et al. 1998), neuronal activity is enhanced (Schiene et al. 1996), NMDA receptor binding is increased (Que et al. 1999) and LTP, a cellular paradigm for learning, is increased (Hagemann et al. 1998; Mittmann \& Eysel 2001). However, in contrast to the hypothesis of facilitated plasticity after stroke (for a review, see also Murphy \& Corbett 2009) an in vivo study provided evidence for diminished plasticity in direct vicinity of a cortical lesion (Jablonka et al. 2007). In these experiments, experience-dependent plasticity of the cortical representation of vibrissae was analyzed after a photothrombotically induced lesion next to the barrel cortex in rats. Plasticity in the barrel cortex was evoked by clipping all but one row of whiskers. After a month of vibrissae deprivation, rats were injected with 2-deoxyglucose,

which was radioactively labeled with ${ }^{14} \mathrm{C}$. Active neurons take up $\left[{ }^{14} \mathrm{C}\right]$-2-deoxyglucose and can be then visualized later on. In control rats, vibrissae stimulation of the untrimmed 
whiskers caused an enlargement of their functional cortical representation. After a focal photothrombotic stroke next to the barrel cortex, no experience-dependent rearrangement of the spared row representation occurred.

It is evident that there must be factors that counteract brain plasticity after stroke. One likely candidate is the inflammatory reaction: stroke initiates an inflammatory reaction at the injured site and surrounding areas (Garcia 1975; Liu et al. 1994; Sairanen et al. 2001; Schroeter et al. 2002; for a review, see Wang et al. 2007; Liguz-Lecznar \& Kossut 2013) and accounts for its progression and even aggravates the induced damage (Barone \& Feuerstein 1999; for reviews, see Dirnagl et al. 1999; Chamorro \& Hallenbeck 2006). Antagonizing this inflammation can reduce lesion size (Cole et al. 1993; Patel et al. 1993; Xu et al. 2004). Thus, post-ischemic inflammation processes represent a promising target for therapeutic interventions in stroke. First encouraging results were obtained in a clinical trial with acute stroke patients where inflammation was encountered with an interleukin-1 receptor antagonist. Clinical outcomes 3 months after stroke were better in the treated group than in a placebo group and no adverse side effects of the drug were observed (Emsley et al. 2005). In our study, the impact of a cortical stroke in mice and the efficacy of anti-inflammatory treatment on the two different plasticity paradigms (OD-plasticity and enhancement of visual abilities after MD) were investigated. Moreover, plasticity at different time points after the PT-lesion was analyzed. It has been suggested that for stroke rehabilitation an early time window should be used. More specifically, fewer days between stroke and the start of rehabilitation was associated with better functional outcome in rats (Biernaskie et al. 2004) as well as in humans (Maulden et al. 2005). However, some studies reported disrupted recovery of function and severe chronic behavioral deficits after early forced training of the impaired limb (Kozlowski et al. 1996). Thus, inflammation and timing of treatment seem to be crucial components of stroke treatment and are further analyzed in this study.

\subsection{Enriched environment}

What other therapeutic options are there to promote plasticity? One appealing, because noninvasive, method is the environmental enrichment (EE). EE refers to a housing condition, which is "enriched", compared to standard laboratory housing conditions. In general, the "enriched" animals are kept in larger cages, with running wheels, frequently changed objects and a larger group of animals (Figure 5). 


\section{standard cage}

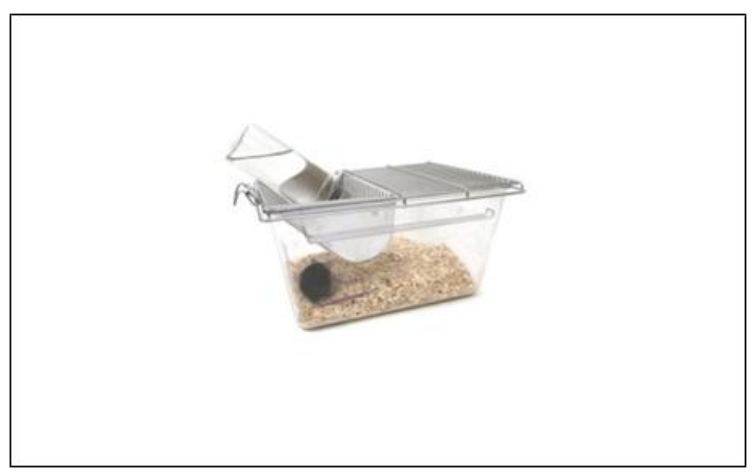

enriched environment

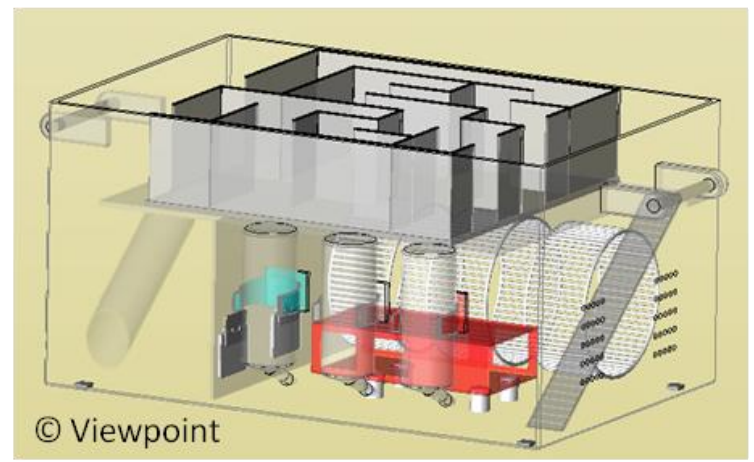

Figure 5: Enriched environment (EE) housing provides complexity, activity, novelty and wellbeing. The standard cage (left) is small, with just one compartment, no toys and only 3-5 of mice housed together. In contrast to that the EE cage (right; Marlau, Viewpoint, Frances) is large with two floors and provided possibilities for enhanced voluntary physical exercise (running wheels), cognitive stimulation (mice have to go through a maze that is changed 3 times a week) and social interaction (up to 16 mice per cage).

Rosenzweig and colleagues established EE as a scientific concept (Rosenzweig et al. 1962; Rosenzweig 1966). The purpose of $\mathrm{EE}$ is to provide possibilities for enhanced voluntary physical exercise, social interactions, multi-sensory and cognitive stimulation. EE has profound effects on the developing and the adult brain, spanning from the molecular to the anatomical and behavioral level (for a review see van Praag et al. 2000; Baroncelli et al. 2010a). In adult animals, EE leads to better performance in various learning and memory tasks like the Morris-water-maze, the novel object recognition task and fear-conditioning task (for a review see Rampon \& Tsien 2000) and reduces the cognitive decline associated with aging (Mohammed et al. 1993; for a review see Mohammed et al. 2002). EE is also capable of delaying the progression and reducing provoked deficits of various neurological pathologies, including neurodegenerative diseases and stroke (for a review, see Nithianantharajah \& Hannan 2006). EE also affects the visual system: it enhances visual system development (Prusky et al. 2000a; Cancedda et al. 2004; Sale et al. 2004) and accelerates eye opening (Cancedda et al. 2004; Sale et al. 2004). This is probably due to increased levels of BDNF (Cancedda et al. 2004; Sale et al. 2004), insulin-like growth factor 1 (IGF-1) (Ciucci et al. 2007; Maya-Vetencourt et al. 2012), enhanced maturation of the inhibitory GABAergic system (Cancedda et al. 2004; Ciucci et al. 2007) and accelerated CRE/CREB (cAMP response element-binding protein)-mediated gene expression (Cancedda et al. 2004). In the adult rodent visual cortex, EE increased levels of BDNF (Sale et al. 2007; Baroncelli et al. 2010b; Tognini et al. 2012) and serotonin (Baroncelli et al. 2010b), decreased GABA levels 
and release (Sale et al. 2007; Baroncelli et al. 2010b; Baroncelli et al. 2012), the number of GAD67 ${ }^{+}$interneurons (Scali et al. 2012; Tognini et al. 2012) and the density of PNNs (Sale et al. 2007; Scali et al. 2012). Moreover, EE also increased levels of histone acetylation in the hippocampus and neocortex (Fischer et al. 2007). Since all of these effects promote plasticity, we wondered whether raising mice in EE could prolong the sensitive phase for OD-plasticity or even restore it in older mice.

In adult rats, EE has already been proven to be effective in reactivating OD-plasticity. In a study of Baroncelli et al. (2010b), rats (PD 60) were housed in an EE for 3 weeks. After 2 weeks of EE, MD was performed for 7 days. In contrast rats housed in SC, the EE-rats showed OD-plasticity, as assessed with visual evoked potentials and single-unit recordings (Baroncelli et al. 2010b). The visual evoked potentials recordings also showed that ODplasticity in EE animals acted through a juvenile-like mechanism: the response to stimulation of the deprived MD eye decreased. Recently, Scali et al. (2012) showed that 3 weeks of EEhousing can also reactivate OD-plasticity in aging rats with an age of 22 to 24 months, using the same EE-housing and measurement protocol like Baroncelli et al. (2010b). Remarkably, $\mathrm{EE}$ is also able to reverse abnormal function of the visual cortex in adult amblyopic rats, due to long-term MD. An exposure to EE of 2 to 3 weeks allowed a full recovery of the visual acuity of the deprived eye and recovery of binocularity after reopening of the deprived eye (Sale et al. 2007; Baroncelli et al. 2012). Moreover, these studies showed reduced extracellular GABA levels (Sale et al. 2007; Baroncelli et al. 2010b) and reduced GABA release (Baroncelli et al. 2012) in EE-housed rats. Enhancement of GABAergic inhibition through diazepam treatment completely abolished the restoration of plasticity (Sale et al. 2007; Baroncelli et al. 2010b), suggesting that the reduced inhibition is a crucial molecular mechanism for the improvement of plasticity induced by EE. In our study, we examined for the first time whether EE can prolong the sensitive phase for OD-plasticity or even restores it in older mice and if this depends on GABAergic inhibition.

Since EE has several positive effects on brain and behavior, various studies investigated its benefit in animal models for Alzheimer's disease, Parkinson's disease, amyotrophic lateral sclerosis, Down syndrome and various forms of brain injury, including stroke (for a review see Nithianantharajah \& Hannan 2006). Several studies demonstrated a positive influence of EE on the outcome after experimental brain damage. For instance, in rodent models of stroke, EE has been shown to promote motor function recovery (Ohlsson \& Johansson 1995; Johansson 1996; Biernaskie et al. 2004; Wurm et al. 2007) and lessen deficits in learning and memory (Dahlqvist et al. 2004; Rönnbäck et al. 2005; Wurm et al. 2007) (meta-analysis in 
Janssen et al. (2010)). However, so far no studies have been done to analyze the potential beneficial effect of EE raising on the stroke induced impairments on visual cortex plasticity in mice. To this end, we additionally tested if EE is capable of preserving plasticity after stroke.

\subsection{Scope of the thesis}

Brain plasticity is most needed when the brain suffers from a sudden lesion, such as following a stroke: the brain needs to adapt and compensatory mechanisms have to be developed. A stroke not only impairs directly affected brain area but also disturbs more distant brain regions. It is therefore essential to support and optimize brain plasticity. In the present study a focal photothrombotically induced cortical stroke was positioned in S1, in the vicinity of V1 in adult mice (C57BL/6J). To induce visual plasticity the well established model of MD was used: one eye was closed for 7 days. Two different measurements of experience- and learning-dependent plasticity were obtained in one and the same animal. First, the enhancement of visual abilities after MD (the increase of visual acuity and contrast sensitivity of the open eye) was measured as a model of sensory learning using the optomotor system (Prusky et al. 2004). Following this behavioral test, OD-plasticity was analyzed as an established model of cortical plasticity using in vivo optical imaging of intrinsic signals (Kalatsky \& Stryker 2003; Cang et al. 2005). We investigated the impact of a PT-lesion on sensory learning, and on OD-plasticity as well as on the activity of V1 in the lesioned and non-lesioned hemisphere. Since stroke is associated with an inflammatory reaction, we tested the efficacy of an anti-inflammatory treatment by daily intraperitoneal injections of ibuprofen during the MD-period. Further, plasticity at different time points after the lesion was tested. For that a delay of 1 or 2 weeks was introduced between PT and MD.

As another therapeutic option to improve plasticity, we used the non-invasive and nonpharmacological method of EE, where mice are housed in larger cages with enhanced possibilities for voluntary physical exercise, social interaction, multi-sensory and cognitive stimulation. The aim of the study was to examine whether EE can prolong the sensitive phase for OD-plasticity, which usually ends at PD 110 in SC-mice (Lehmann \& Löwel 2008). For that, mice were raised in the EE until they were 130 days old, or even older. Moreover, we wanted to test whether EE acts via modification of inhibition. To do so, EE-mice were treated with daily injections of diazepam (positive allosteric $\mathrm{GABA}_{\mathrm{A}}$ receptor modulator) during the MD-period. Since PV-positive cells and PNNs play an important role in OD-plasticity, the number of PV-positive inhibitory interneurons and PNNs (WFA-staining) in V1 were 
quantified by means of immunofluorescence in SC- and EE-mice. To test if EE-housing can even restore OD-plasticity in older mice, we transferred SC-mice at PD 110, an age where no OD-plasticity is inducible any more in the imaging experiments, into an EE-housing. Moreover, we explored whether $\mathrm{EE}$ was capable of preserving plasticity after a photothrombotic stroke.

An overview scheme of the main points addressed in this thesis can be found in Figure 6.

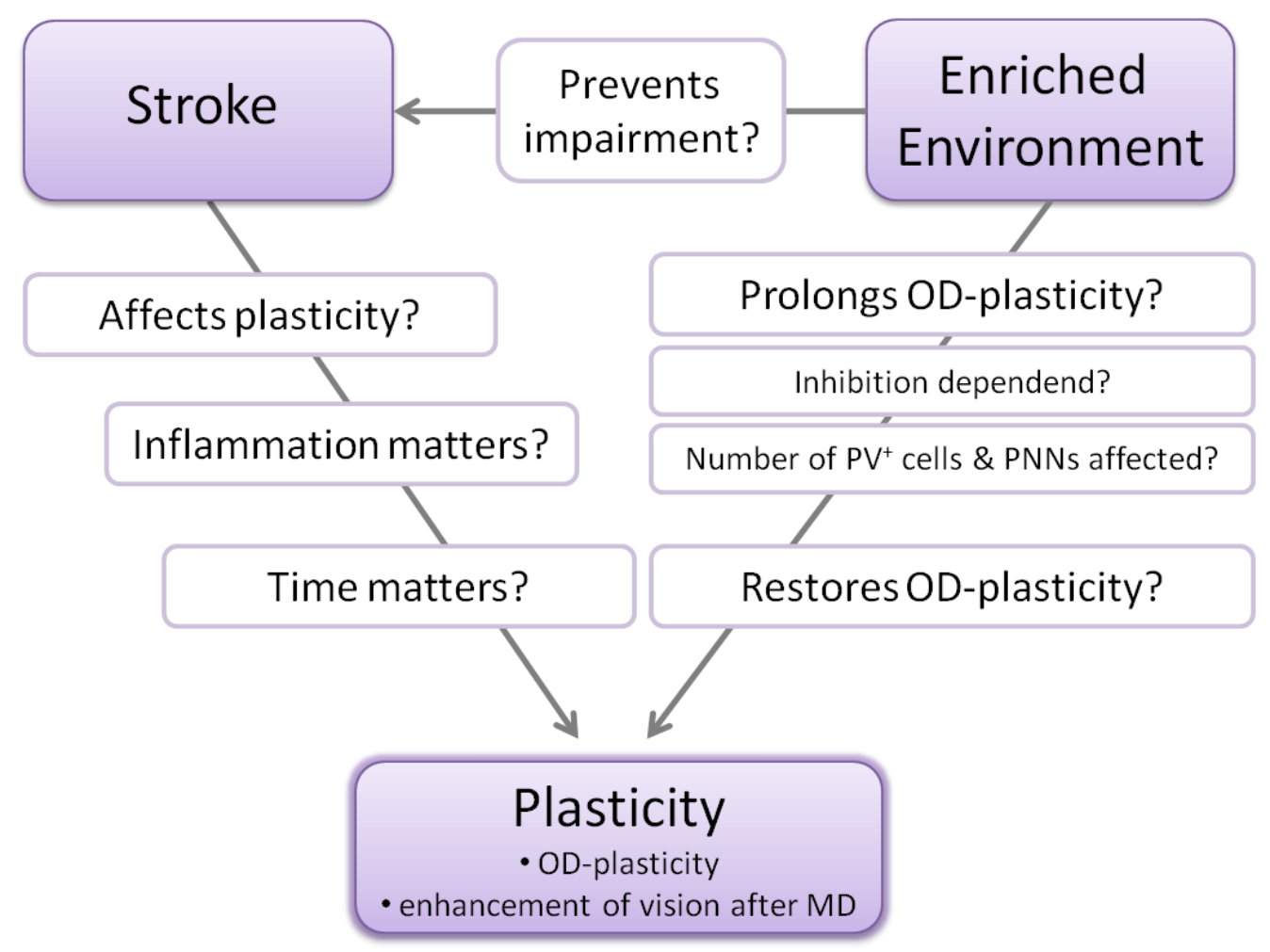

Figure 6: Overview scheme of the main points addressed in the thesis. Brain plasticity is crucial for rehabilitation after stroke. The present study was designed to investigate the impact of a focal photothrombotically induced cortical stroke on plasticity of the neighboring visual cortex in the lesioned hemisphere and in the non-lesioned hemisphere (left). As plasticity measurements we assessed OD-plasticity and the enhancement of vision after MD ("sensory learning") in an in vivo approach in mice. We also tested whether a time delay between PT and MD would affect visual plasticity. Since stroke is associated with an inflammatory reaction, the efficacy of anti-inflammatory treatment with ibuprofen was tested. As another therapeutic option to improve plasticity we used EEhousing to enhance voluntary physical exercise, social interactions, multi-sensory and cognitive stimulation (right). We wanted to examine whether raising mice in EE could prolong the sensitive phase for OD-plasticity and if this depends on inhibition. Moreover, inhibitory PV-positive neurons and PNNs where quantified after EE raising. Another aim was to find out whether EE can even restore OD-plasticity in older mice. Additionally, we tested whether EE can preserve plasticity after stroke (top). 


\section{MATERIAL AND METHODS}

A list of the used chemicals, drugs and substances together with the producing companies can be found at the end of the chapter (section 2.15, page 49).

\subsection{Animals}

All experiments were performed with C57BL/6J mice. Mice for the stroke study were obtained from the animal facility of the Forschungszentrum Lobeda (Hans-Berger Klinik für Neurologie, Universitätsklinikum Jena FZL). Experiments were approved by the local government “Thüringer Landesamt für Lebensmittelsicherheit und Verbraucherschutz" under the registration number 02-003/08. Enriched environment (EE) experiments were partly (PD 130 group) carried out with mice of the breeding colony of the Institut für Allgemeine Zoologie und Tierphysiologie of the Friedrich-Schiller-University Jena, approved by "Thüringer Landesamt für Lebensmittelsicherheit und Verbraucherschutz" under the registration number 02-015/06. Mice for the other EE groups were obtained from the central animal facility of the University Medical Center Göttingen. Experiments were approved by „Niedersächsisches Landesamt für Verbraucherschutz und Lebensmittelsicherheit“" under the registration number 33.9-42502-04-10/0326. Mice were housed in an animal room with a 12 hours light/dark cycle, with food and water available ad libitum.

\subsection{Study design}

All animals were first tested daily in the optomotor setup (measurement of visual acuity and contrast sensitivity). Then their visual cortical activity and ocular dominance (OD) plasticity was analyzed in vivo by optical imaging of intrinsic signals. Monocular deprivations (closure of one eye, MD) to induce plasticity lasted 7 days. At the end of the optical imaging experiments all animals were perfused in order to analyze the photothrombotic (PT) lesion and quantify parvalbumin (PV)-positive cells and perineuronal nets (PNNs) in the respective groups.

\section{$\underline{\text { Stroke study }}$}

We investigated the impact of a cortical stroke (PT) on the increase of visual abilities and OD-plasticity in the lesioned and non-lesioned hemisphere after MD. The effect of antiinflammatory treatment with ibuprofen as well as a delay between stroke induction and 
induction of plasticity by MD was tested. Male mice raised in standard cages (SC) with an age of postnatal day (PD) 70 to 110 at the day of optical imaging were used.

Different experimental groups were treated as follows. Control groups were sham-operated on day 0 (Table 1; Figure 7 A, B), which means that they got the identical treatment as mice in the PT-group (surgery, anesthesia etc.) but the light source to induce photothrombosis was not switched on. About 4 to 5 hours after the sham/PT surgery, animals received either MD of the right eye or no MD (Figure 7 A). The PT-lesion was positioned in the left hemisphere. Some PT-mice with MD were also treated with a daily intraperitoneal injection of ibuprofen to antagonize inflammation or $\mathrm{NaCl}$ as a control, respectively (Figure $7 \mathrm{~B}$ ). To test whether ibuprofen itself had an effect on visual abilities or OD, sham-operated mice were treated with ibuprofen (Figure $7 \mathrm{~B}$ ). In order to test whether the PT-lesion just had an acute effect or causes longer lasting modifications in the brain, a delay of 1 or 2 weeks was introduced between stroke induction and MD (= late MD) (Figure $7 \mathrm{C}$ ). Additional groups of control, PT, $\mathrm{PT}+\mathrm{MD}+\mathrm{Ibu}$ and PT+lateMD (2w) mice received MD of the left eye to preferentially challenge OD-plasticity in the right hemisphere.

Table 1: Experimental groups of the stroke study. Listed is their individual treatment. All mice were male and aged between 70 and 110 days at the day of optical imaging. MD lasted always 7 days. $\mathrm{w}=$ week

\begin{tabular}{|c|c|c|c|}
\hline Group & Sham/PT & MD & NaCl/lbuprofen \\
\hline Control & Sham & - & - \\
\hline Control+MD & Sham & MD right eye & - \\
\hline PT & PT & MD left eye & - \\
\hline PT+MD & PT & - & - \\
\hline Control+Ibu & Sham & MD right eye & - \\
\hline PT+MD+Ibu & PT & MD left eye & Ibuprofen \\
\hline PT+MD+NaCl & PT & MD right eye & Ibuprofen \\
\hline PT+lateMD (1w) & PT & MD left eye & ND right eye \\
\hline PT+lateMD (2w) & & MD right eye & NaCl \\
\hline & PT & MD right eye & \\
\hline
\end{tabular}



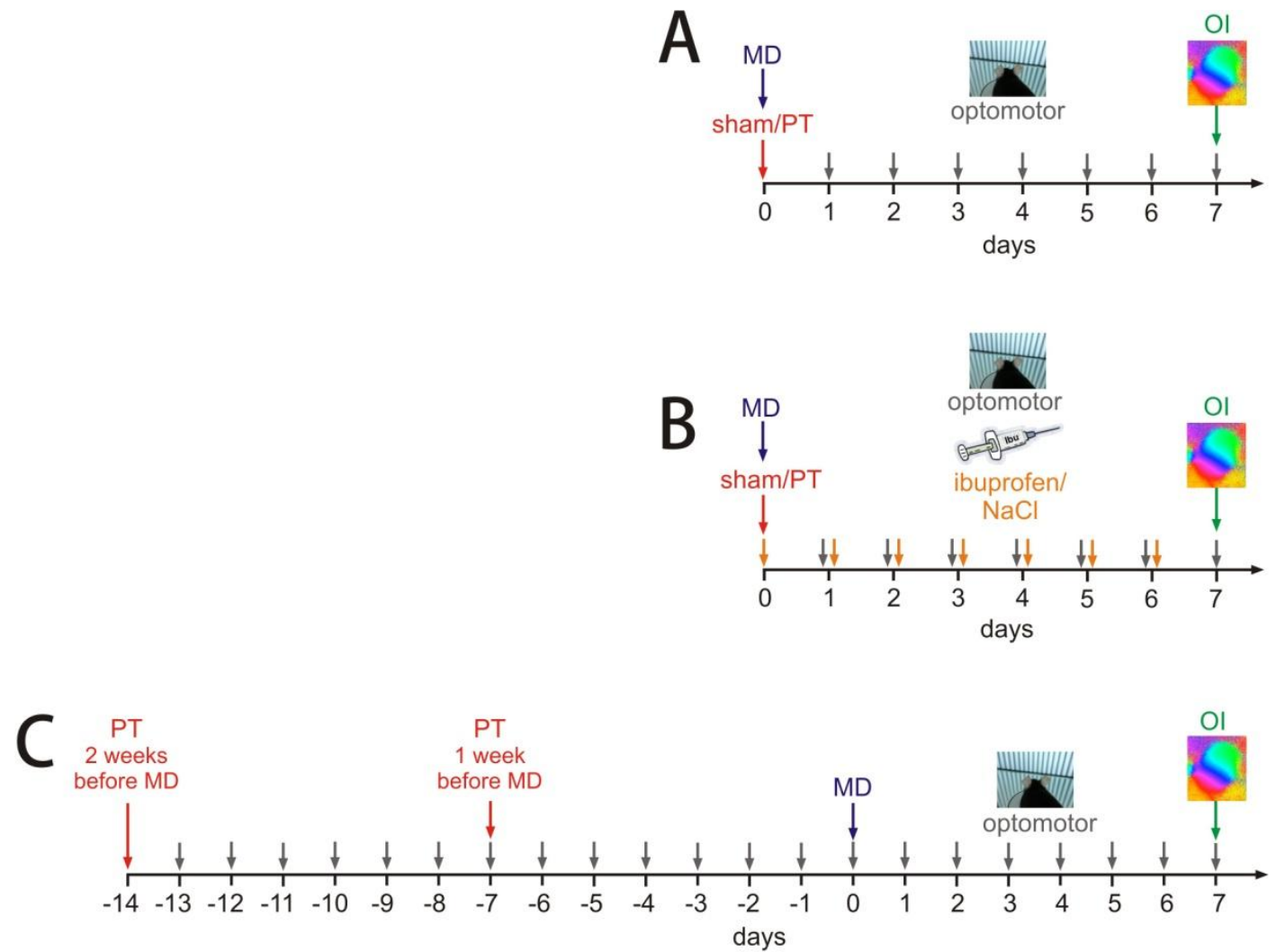

Figure 7: Timeline of stroke experiments. (A) PT or sham treatment on day 0, and MD. Visual abilities were measured daily with the optomotor setup starting on day 1 . On day 7, optical imaging of intrinsic signals (OI) was performed. Groups: control, control+MD, PT, PT+MD. (B) Groups in which mice were treated daily with ibuprofen/NaCl. Groups: control+Ibu, PT+MD+Ibu, $\mathrm{PT}+\mathrm{MD}+\mathrm{NaCl}(\mathrm{C})$ late MD paradigm: MD is induced 1 or 2 weeks after PT. Groups: PT+lateMD (1w), PT+lateMD (2w).

\section{Enriched environment study}

Aim of this part of the study was to test (i) whether EE-housing can prolong the sensitive phase for OD-plasticity, (ii) if EE acts via modification of inhibition, (iii) EE affects the number of PV-positive cells and PNNs and (iiii) if EE can restore OD-plasticity in SC-mice that were already beyond their "plastic" phase (= late EE). Moreover, it was tested whether EE can prevent deficits due to a PT stroke lesion.

To this end, pregnant dams were put into an EE cage (Marlau, Viewpoint, France) about 1 week before delivery and pups were weaned and separated by females and males at PD 30. Both female and male mice were used for the experiments. Mice were raised in the EE until they reached $\geq$ PD 130 (Figure 8), an age at which mice raised in SC do not show ODplasticity any more as visualized by intrinsic signal optical imaging (Lehmann \& Löwel 2008). One part of the animals received MD, the other part did not (Table 2). Within this group (EE since PD -7) we analyzed two different age ranges. Mice in the PD 130 group were between 119 and 136 days old. Mice in the PD 220 group: 177 to 266 days. The PD 130 
group experiments were performed by $\mathrm{PhD}$ student Katja Krempler at the Friedrich-SchillerUniversity Jena. As a control, we used mice that were kept in SC for their whole life. Likewise one group received MD, the other did not. In order to investigate whether the changes induced by EE depend on inhibition, a group of EE-mice and SC-mice were treated daily during the MD-period with diazepam (1mg/kg mouse, intraperitoneally), a positive allosteric $\mathrm{GABA}_{\mathrm{A}}(\gamma$-aminobutyric acid) receptor modulator to increase inhibition. In SCmice (PD 235, no MD) and EE-mice (PD 250, no MD) PV-positive cells and PNNs were quantified by means of immunofluorescence in the primary visual cortex (V1). For the late EE paradigm, mice were raised in a SC and transferred to the EE at PD 110 (Figure 8). Mice were kept in the EE for about 3 to 7 months. Again one group received MD, the other not (Table 2). To test EE as a prevention method for deficits due to a PT stroke, mice were raised in EE until adulthood (PD 90), before exposing them to a PT-lesion and MD/ no MD (Table 2, Figure 8). The indicated ages in the text are at the day of optical imaging.

Table 2: Experimental groups for the EE study. Listed is their individual treatment, age range (in days, at the day of optical imaging) and sex (M=male, $\mathrm{F}=$ female). MD lasted always 7 days. $\mathrm{DZ}=$ diazepam.

\begin{tabular}{|c|c|c|c|c|c|c|}
\hline Group & SC/EE & PT & Diazepam & MD & Age [d] & Sex \\
\hline SC & $\mathrm{SC}$ & - & - & - & \multirow{2}{*}{$222-249$} & \multirow{2}{*}{ M } \\
\hline SC+MD & $\mathrm{SC}$ & - & - & MD right eye & & \\
\hline $\begin{array}{c}\mathbf{E E} \\
\text { (PD 130) }\end{array}$ & $\begin{array}{c}\mathrm{EE} \\
\text { since PD -7 }\end{array}$ & - & - & - & \multirow{2}{*}{$119-136$} & \multirow{2}{*}{$\mathrm{M}+\mathrm{F}$} \\
\hline $\begin{array}{l}\text { EE+MD } \\
\text { (PD 130) }\end{array}$ & $\begin{array}{c}\text { EE } \\
\text { since PD -7 }\end{array}$ & - & - & MD right eye & & \\
\hline $\begin{array}{c}\mathbf{E E} \\
\text { (PD 220) }\end{array}$ & $\begin{array}{c}\mathrm{EE} \\
\text { since PD -7 }\end{array}$ & - & - & - & \multirow{2}{*}{$177-266$} & \multirow{2}{*}{$\mathrm{M}+\mathrm{F}$} \\
\hline $\begin{array}{l}\text { EE+MD } \\
(\text { PD 220) }\end{array}$ & $\begin{array}{c}\mathrm{EE} \\
\text { since PD -7 }\end{array}$ & - & - & MD right eye & & \\
\hline $\mathrm{SC}+\mathrm{DZ}+\mathrm{MD}$ & SC & - & Diazepam & MD right eye & $76-91$ & \multirow[b]{2}{*}{$\mathbf{M}$} \\
\hline $\mathbf{E E}+\mathrm{DZ}+\mathbf{M D}$ & $\begin{array}{c}\mathrm{EE} \\
\text { since PD -7 }\end{array}$ & - & Diazepam & MD right eye & $162-190$ & \\
\hline Late EE & $\begin{array}{c}\mathrm{EE} \\
\text { since PD } 110\end{array}$ & - & - & - & \multirow{2}{*}{$212-345$} & \multirow{2}{*}{$\mathrm{F}$} \\
\hline Late $\mathbf{E E}+\mathbf{M D}$ & $\begin{array}{c}\text { EE } \\
\text { since PD } 110\end{array}$ & - & - & MD right eye & & \\
\hline $\mathbf{E E}+\mathrm{PT}$ & $\begin{array}{c}\text { EE } \\
\text { since -PD } 7\end{array}$ & $\mathrm{PT}$ & - & - & \multirow{2}{*}{$80-114$} & \multirow{2}{*}{$\mathrm{M}+\mathrm{F}$} \\
\hline $\mathbf{E E}+\mathrm{PT}+\mathrm{MD}$ & $\begin{array}{c}\text { EE } \\
\text { since -PD } 7\end{array}$ & PT & - & MD right eye & & \\
\hline
\end{tabular}




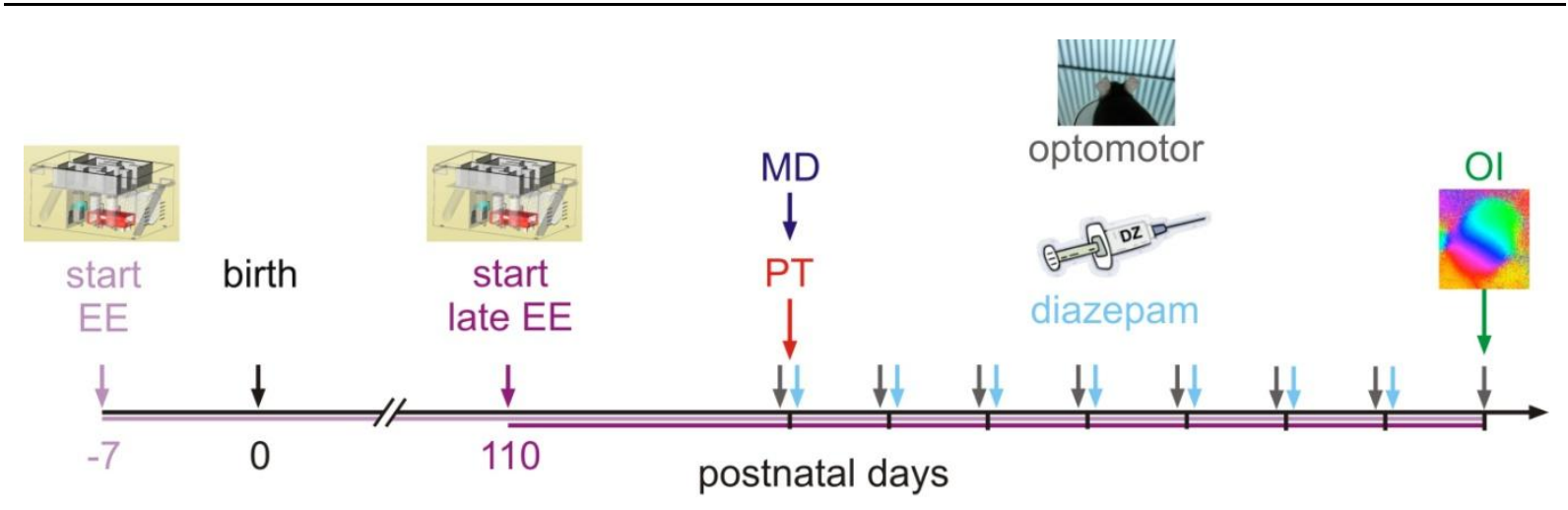

Figure 8: Timeline of EE experiments. EE-housing started before birth. One group was treated with diazepam for the duration of MD. Another group of mice received a PT on the day of MD. Late EE started from PD 110 on. Visual abilities were measured daily with the optomotor setup starting at the day of MD. Seven days later, optical imaging of intrinsic signals was performed.

\subsection{Enriched environment housing}

The EE-housing condition (Marlau cage, Viewpoint, www.viewpoint.fr, France; Figure 9) should enhance physical exercise, social interaction and cognitive and sensory stimulation. The EE consists of a large cage $(56 \times 37 \times 32 \mathrm{~cm})$ with two floors linked by a ladder for going up and a tube for sliding down. In the upper compartment a maze is integrated, which was changed 3 times a week. A total of 12 different maze configurations are possible. In the lower compartment, the "living area", water, 3 running wheels for voluntary physical exercise, and a red tunnel to protect the mice from light during their resting periods are provided. The food was placed in a separate compartment. In order to move from the "living area" to the "food area", mice have to go up the ladder, through the labyrinth and down the slide. Once they go back into the "living area" through a revolving door which just opens in one direction they are forced to move through the labyrinth again in order to get food. Food and water were available ad libitum. Social interactions are promoted by the possibility to house large number of mice together (up to 16). Thus, the EE condition offers complexity, activity and novelty. Pregnant dams were put into the EE about 1 week before giving birth. For the late EE paradigm, mice were raised in a SC and transferred to the EE at PD 110.

In contrast to EE cages, standard cages consisted of a much smaller cage $(26 \times 20 \times 14 \mathrm{~cm})$, with just one compartment, no toys and only 3-5 mice housed together (Figure 9). 


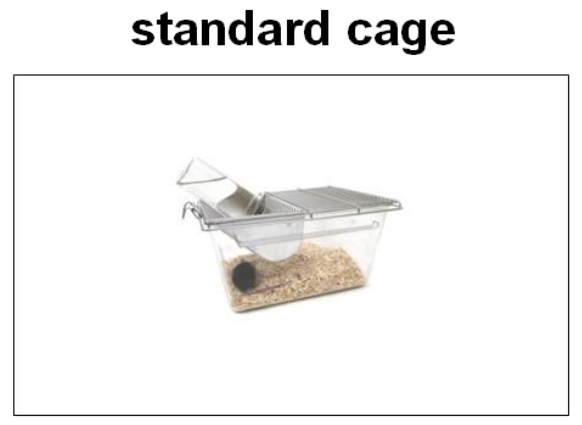

\section{enriched environment}
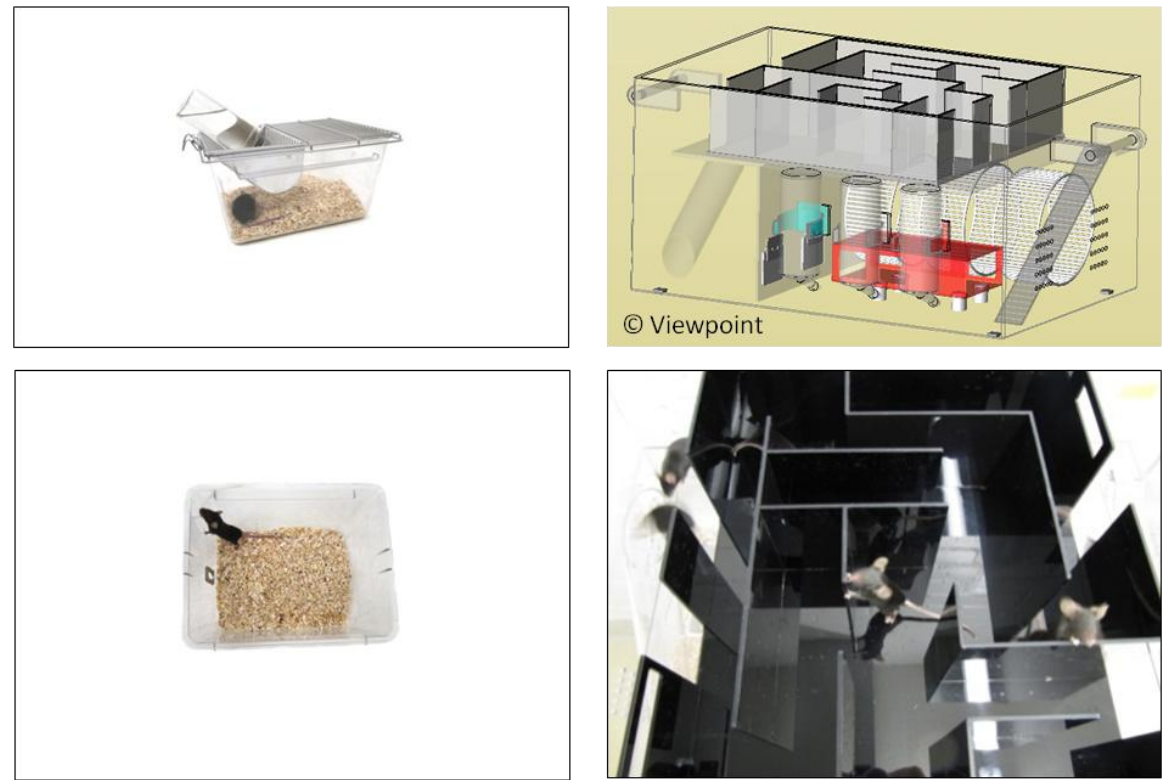

Figure 9: Standard cage versus enriched environment. The standard cage (left) is small, with just one compartment, no toys and only 3-5 of mice housed together. In contrast to that the EE cage (right; Marlau, Viewpoint, Frances) offers complexity, activity, novelty and wellbeing. The cage is large with two floors and provided possibilities for enhanced voluntary physical exercise (running wheels), cognitive stimulation (mice have to go through a maze that is changed 3 times a week) and social interaction (a large number of mice is housed together).

\subsection{Photothrombotic stroke lesion}

As a stroke model we used the photothrombosis technique developed by Watson et al. (1985). With this technique a local cortical lesion was induced in the left somatosensory cortex. The main advantages of this technique are the precise and restricted localization of the lesion and its reproducibility (Witte \& Stoll 1997). The lesion can be positioned quite specifically by stereotaxic coordinates and can be restricted to the cortex, so that subcortical brain regions are not affected (Domann et al. 1993). Additionally, the surgery which is required for the induction of the lesion is minimally invasive. Since in this study a well described cortical lesion should be induced next to the visual cortex, the PT model was chosen over other stroke models (review on stroke models Krafft et al. 2012).

\section{Surgery}

Mice received an intravenous injection of the photosensitive dye rose bengal and the brain region where the lesion should be localized was illuminated through the intact skull with a focused cold light source. This causes a photochemical reaction that leads to endothelial 
damage which induces platelet aggregation in brain vessels and leads to an ischemic infarct with cell death in the illuminated area (Watson et al. 1985).

All PT surgeries, except for the EE study, were performed by technician Svetlana Tausch in the Hans-Berger-Klinik für Neurologie, Universitätsklinikum Jena, Germany.

Mice were initially anesthetized in a box with $2 \%$ isoflurane in a mixture of $3: 1 \mathrm{~N}_{2} \mathrm{O}: \mathrm{O}_{2}$ and anesthesia was maintained during surgery with an inhalation mask. The animals were placed in a stereotaxic frame and the body temperature was kept at $37^{\circ} \mathrm{C}$ using a heating pad with a feedback mechanism (FHC, Bowdoinham, Maine, USA). Eyes were covered with eye gel and aluminum foil to protect them from light and drying-out. The skull was made accessible by incising the skin above. An optic fiber bundle $(\varnothing 1,0 \mathrm{~mm})$ mounted on a cold light source was positioned $2 \mathrm{~mm}$ lateral to the midline and $1 \mathrm{~mm}$ posterior to the bregma (Figure 10).

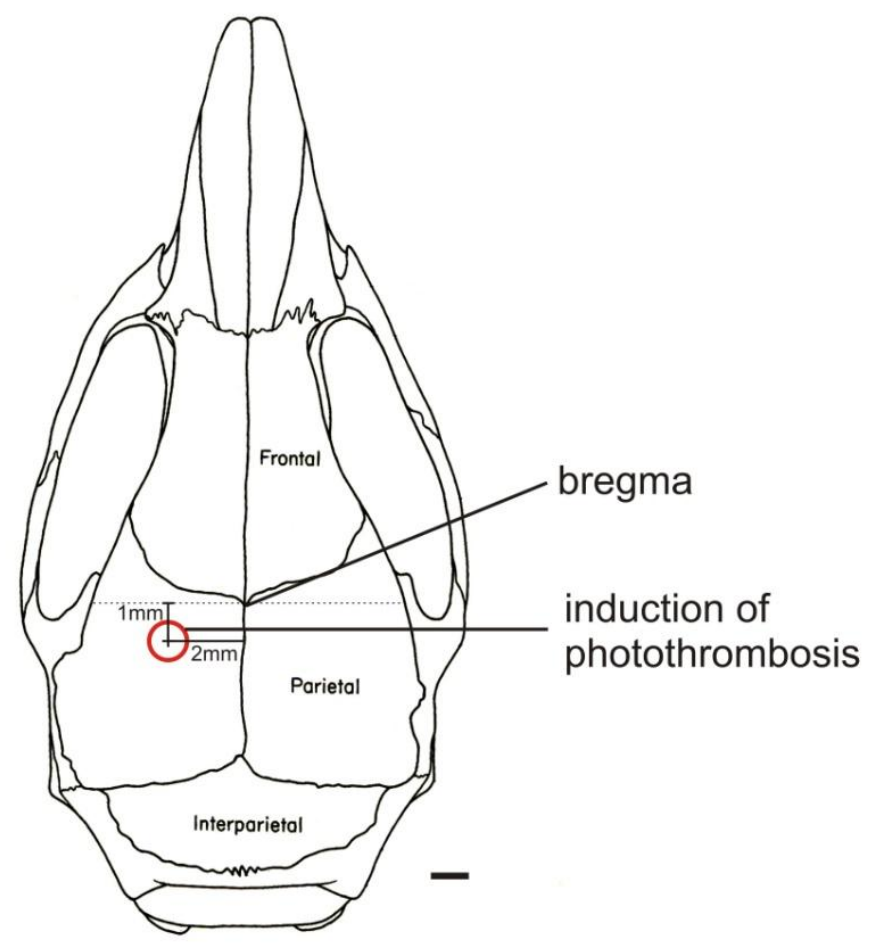

Figure 10: Schematic representation of the position of the induction of photothrombosis on the skull of a mouse. Scale bar: $1 \mathrm{~mm}$. Modified from Cook (1965).

Next, $100 \mu \mathrm{l}$ rose bengal (concentration: $10 \mathrm{mg} / \mathrm{ml}$, in $0.9 \% \mathrm{NaCl}$ ) were injected into the tail vein. After a waiting period of 5 minutes, the cold light source (KL 1500, Schott) was 
switched on for 15 minutes. After that, the skin was sutured, antiseptic braunovidon gel (with $10 \%$ povidone-iodine) was applied on the suture and animals were allowed to recover in their cage. The control animals were treated identically but the light source was not switched on (sham treatment). Lesion size and location were measured after the optomotor and optical imaging experiments on Nissl-stained frontal brain sections.

\subsection{Visual acuity and contrast sensitivity}

The optokinetic reflex and consequently visual acuity and contrast sensitivity were measured using the fast and easy measurement with the virtual reality optomotor system developed by Prusky et al. (2004). Briefly, the virtual reality system consists of 4 flat-screen monitors facing inwards and creating an arena (Figure 11).

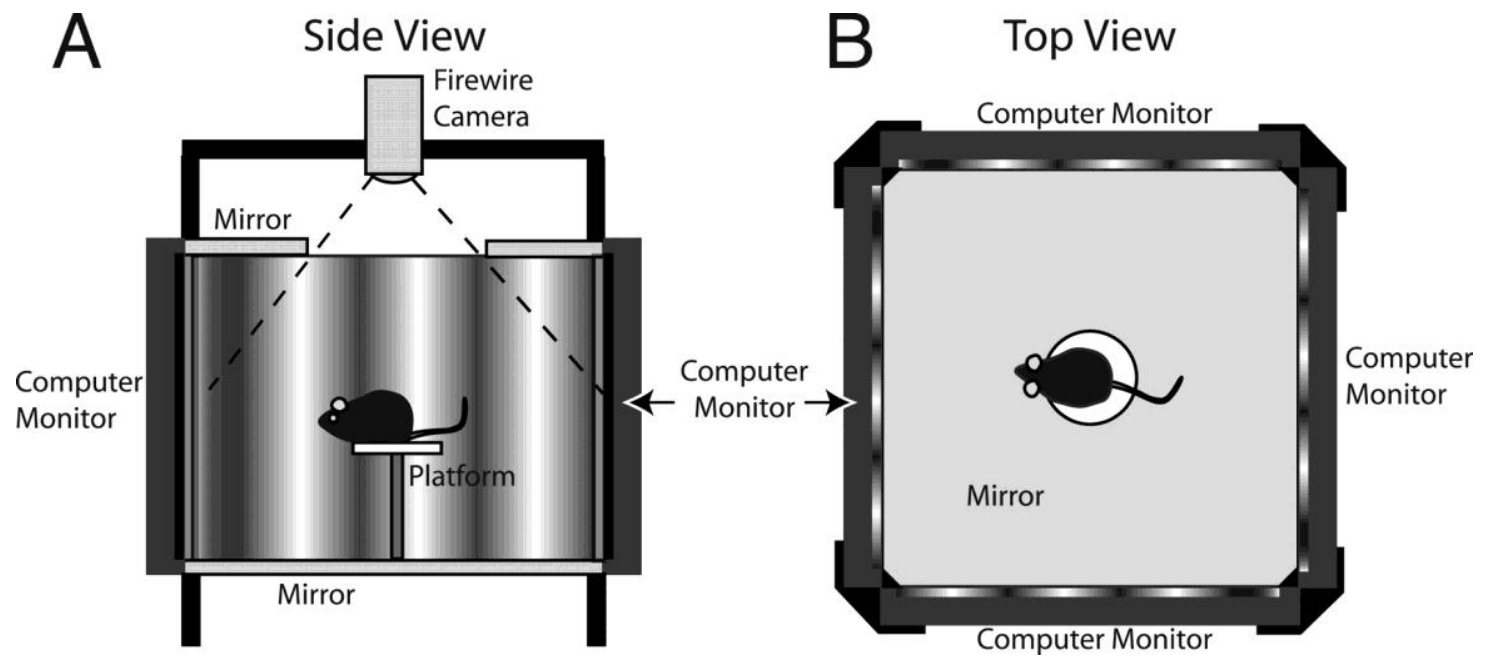

Figure 11: The optomotor testing apparatus. (A) Side view. A mouse is positioned on the platform in the middle of the testing arena, surrounded by computer monitors, on which a sine wave grating is projected. The video camera is used to watch the behavior of the mouse. (B) Top view. The mouse is surrounded by the grating and can move freely on the platform. From Prusky et al. (2004).

A small, elevated platform on which the mouse is positioned and can move freely is positioned in the center of the arena. A virtual cylinder with a vertical sine wave grating is projected on the monitors (Figure 12). The virtual cylinder can rotate clockwise or counterclockwise. Above the platform in the lid of the box, there is a camera mounted to monitor the behavior of the mouse. The center of the rotating virtual cylinder was always positioned between the eyes of the mouse, to make sure, that the walls of the virtual cylinder 
had always the same distance to the eyes of the mouse. With the help of software (OptoMotry 1.4.0, CerebralMechanics, Lethbride, Alberta, Canada) parameters like spatial frequency, contrast and speed of the sine wave grating can be varied. For our experiments we used a rotation speed of $12 \%$.
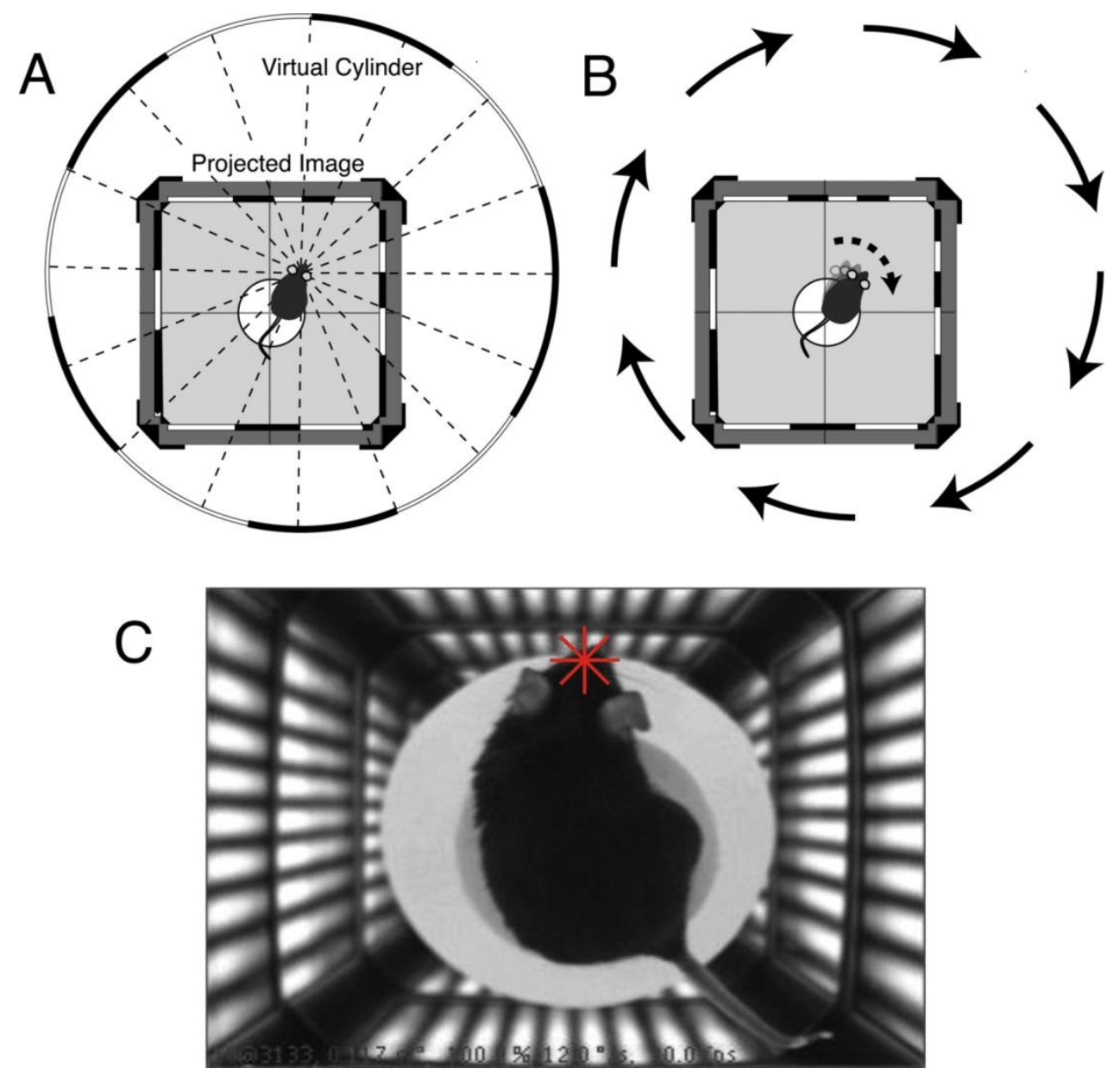

Figure 12: Virtual reality cylinder. (A) The projection of the virtual cylinder in a 3-D coordinate space on the monitors. The cylinder is centered on the eyes of the mouse. (B) When the virtual cylinder with the grating is rotating, the mouse is following with reflexive head and neck movements in the direction of rotation. In this example the movement is mediated by the left eye because the reflex is just provoked by a temporal-nasal movement of the grating. (C) A video camera picture of a mouse tracking the cylinder grating. The red cross-hair is used to position the center of the rotation of the cylinder between the eyes of the mouse. Modified from Prusky et al. (2004).

Mice will reflexively track the grating by head movements as long as they can see the grating. This optomotor reflex is mediated through subcortical structures. It is generated by relays formed between the accessory optic system (AOS), the nucleus of the optic tract, the ventral tegmental relay zone, the inferior olive and the vestibulocerebellum (Giolli et al. 2005). Thus, the exclusively cortical lesion should not affect these subcortical structures. Visual acuity was 
measured at full contrast and contrast sensitivity at 6 different spatial frequencies [0.031, 0.064, 0.092, 0.103, 0.192, 0.272 cycles/degree (cyc/deg)] (Figure 13).

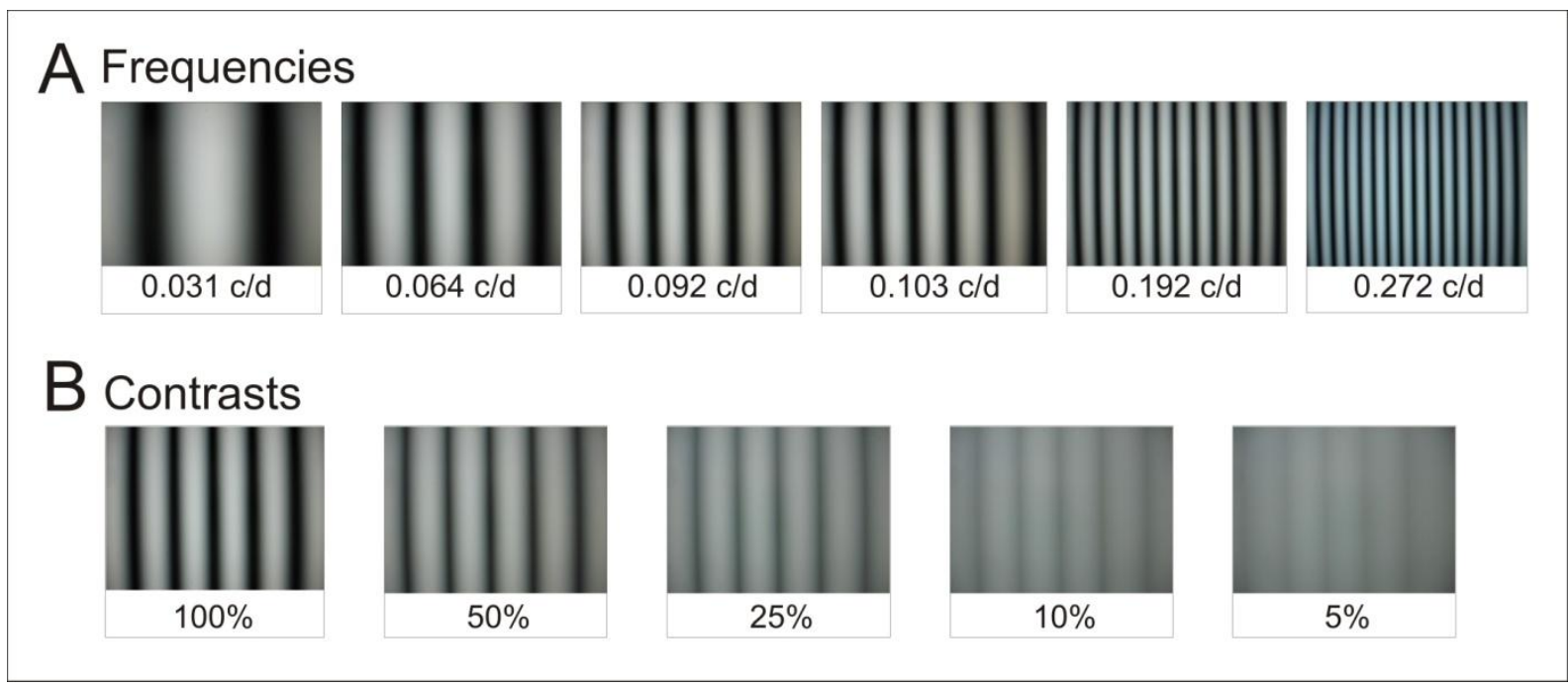

Figure 13: Sine wave gratings in the optomotor system. (A) Different spatial frequencies (in cycles/degree) at $100 \%$ contrast: the higher the spatial frequency, the closer the stripes. Contrast sensitivity was measured at the depicted spatial frequencies. (B) Different contrasts (in \%) at a spatial frequency of $0.092 \mathrm{cyc} / \mathrm{deg}$.

Spatial frequency or contrast was varied by the experimenter, respectively until the threshold of tracking was determined. Because only moving gratings in the temporal-to-nasal direction provoke tracking (Douglas, 2005), it is possible to measure thresholds for both eyes separately. Thus, if the cylinder with the grating is moving clockwise, the resulting reflex is evoked by a reflex circuit through the left eye and vice versa. Contrast sensitivity thresholds measured in percent were converted into Michelson contrasts based on screen luminance $(\max -\min ) /(\max +\min )$ by multiplying with a factor of 0.997 for the proportion of minimal and maximal luminance. Contrast sensitivity values were calculated by dividing 100 by these percentage values. The contrast sensitivity was then plotted against spatial frequency on a $\log -\log$ graph.

\subsection{Monocular deprivation}

To induce plasticity, a MD for 7 days was performed according to published protocols (Gordon \& Stryker 1996; Cang et al. 2005; Lehmann \& Löwel 2008). Briefly, mice were anesthetized with $3.5 \%$ halothane in $1: 1 \mathrm{O}_{2}: \mathrm{N}_{2} \mathrm{O}$ and anesthesia was maintained during 
surgery with $0.6 \%$ to $0.8 \%$ halothane in $1: 1 \mathrm{O}_{2}: \mathrm{N}_{2} \mathrm{O}$ using an inhalation mask. Lid margins were trimmed and an antibiotic gel was applied (Gentamycin gel, active agent: gentamicin sulfat, $0.3 \%$ ). The eye was closed with two mattress sutures using 7-0 silk (Ethicon, Norderstedt), opposing the full extent of the trimmed lids. Mice received a subcutaneous injection of $0.2 \mathrm{ml}$ rimadyl (active agent: carprofen $(50 \mathrm{mg} / \mathrm{ml}$ ) for pain relief. The eyes were checked daily to make sure that they remained closed. In mice for the EE study (accept the PD 130 group) isoflurane anesthesia was used instead of halothane anesthesia.

\subsection{Ibuprofen administration}

In order to suppress inflammatory responses after stroke, we applied ibuprofen, a nonselective cyclooxygenase (COX) inhibitor. Ibuprofen was injected daily intraperitoneally: 60 $\mathrm{mg} / \mathrm{kg}$ (Ibuprofen sodium salt, Fluka) dissolved in $\mathrm{NaCl}(7.5 \mathrm{~g}$ ibuprofen $/ \mathrm{ml} \mathrm{NaCl})$ or $0.9 \%$ $\mathrm{NaCl}$ as vehicle. The first dose was applied directly after MD (day 0) and then daily until day 6.

\subsection{Diazepam administration}

Nine mice reared in the EE were treated with diazepam (Rotexmedica, $1 \mathrm{mg} / \mathrm{kg}$ mouse, intraperitoneally), a positive allosteric $\mathrm{GABA}_{\mathrm{A}}$ receptor modulator, to increase inhibition, starting 4 hours before MD and continued for 6 days with a daily injection. As a control group 3 months old SC-mice were also treated with diazepam in the same manner.

\subsection{Optical imaging of intrinsic signals}

To visualize and register visual cortex activity and acquire the ocular dominance index (ODI) and therefore the OD-plasticity, the in vivo technique of optical imaging of intrinsic signals (Blasdel \& Salama 1986; Grinvald et al. 1986; Kalatsky \& Stryker 2003) was used.

\section{$\underline{\text { Surgical preparations }}$}

Surgical preparations were carried out as described by Kalatsky \& Stryker (2003) and Cang et al. (2005) with small modifications. After initial box-anesthesia with $2 \%$ halothane in 1:1 $\mathrm{O}_{2}: \mathrm{N}_{2} \mathrm{O}$, mice received an intraperitoneal injection of pentobarbital $(50 \mathrm{mg} / \mathrm{kg}, 5 \mathrm{mg} / \mathrm{ml}$ in 
$0.9 \% \mathrm{NaCl}$ ) for additional anesthesia. To dilate both pupils evenly and prevent secretion in the respiratory tract $12 \mathrm{mg} / \mathrm{kg}$ atropine $(0.03 \mathrm{ml}$ from a ready-made $10 \mathrm{mg} / \mathrm{ml}$ solution per mouse) were injected subcutaneously. An injection of dexa-ratiopharm (subcutaneous, active agent: dexamethasone; $8 \mathrm{mg} / \mathrm{kg}, 0.05 \mathrm{ml}$ from a ready-made $4 \mathrm{mg} / \mathrm{ml}$ solution per mouse) helped reducing cerebral edema and an injection of the sedative chlorprothixene (intramuscularly, $8 \mathrm{mg} / \mathrm{kg}, 0.05 \mathrm{ml}$ from a solution of $4 \mathrm{mg}$ chlorprothixene diluted in $1 \mathrm{ml}$ bidest. water) was administered to supplement the anesthesia. Additionally, a local anesthetic (lidocain gel, $2 \%$ ) was applied before all incisions. The temperature of the animal was maintained at $37^{\circ} \mathrm{C}$ by a rectal thermo probe with feedback mechanism to a heating pad (FHC, Bowdoinham, Maine, USA). Animal eyes were kept covered with silicon oil and aluminum foil throughout the surgical procedure to prevent drying-out. Eye drops (Corneregel Fluid) were applied during the experiment as well. A tracheotomy was performed to keep the animals' airway patent and then the animal was placed in a stereotaxic frame. Electrocardiograph leads were attached to the right hind limb and left front limb to monitor the heart rate throughout the experiment. Anesthesia was maintained with $0.6 \%$ halothane in 1:1 $\mathrm{O}_{2}: \mathrm{N}_{2} \mathrm{O}$ applied through the tracheal tube. The skin above the skull was incised to expose the visual cortex. Imaging was performed through the intact skull. Agarose $(2.5 \%$ in $0.9 \%$ $\mathrm{NaCl}$ ) and a glass coverslip were placed over the exposed area to prevent the skull from drying-out and create a suitable optical surface. A subcutaneous injection of $0.2 \mathrm{ml} 0.9 \%$ $\mathrm{NaCl}$ assured hydration of the mouse. In mice from the EE study no tracheotomy was performed and therefore also no pentobarbital was injected.

\section{$\underline{\text { Imaging procedures and visual stimuli }}$}

Intrinsic cortical responses were recorded with the intrinsic signal optical imaging setup developed by Kalatsky and Stryker (2003) and optimized for the assessment of OD-plasticity by Cang et al. (2005).

The intrinsic signal has different components which originate from different sources (reviewed in Bonhoeffer \& Grinvald 1996; Grinvald et al. 1999). One main component of the signal derives from the change in reflectance of cortical vessels due to higher light absorption of deoxyhemoglobin (Figure 14). Neuronal activity in response to visual stimulation is accompanied by metabolic activity and changes in blood volume. Active neurons consume oxygen at higher rates than inactive ones. At an illumination of $610 \mathrm{~nm}$, deoxygenated blood (deoxyhemoglobin) absorbs more light and therefore reflects less light than oxygenated blood (oxyhemoglobin). 


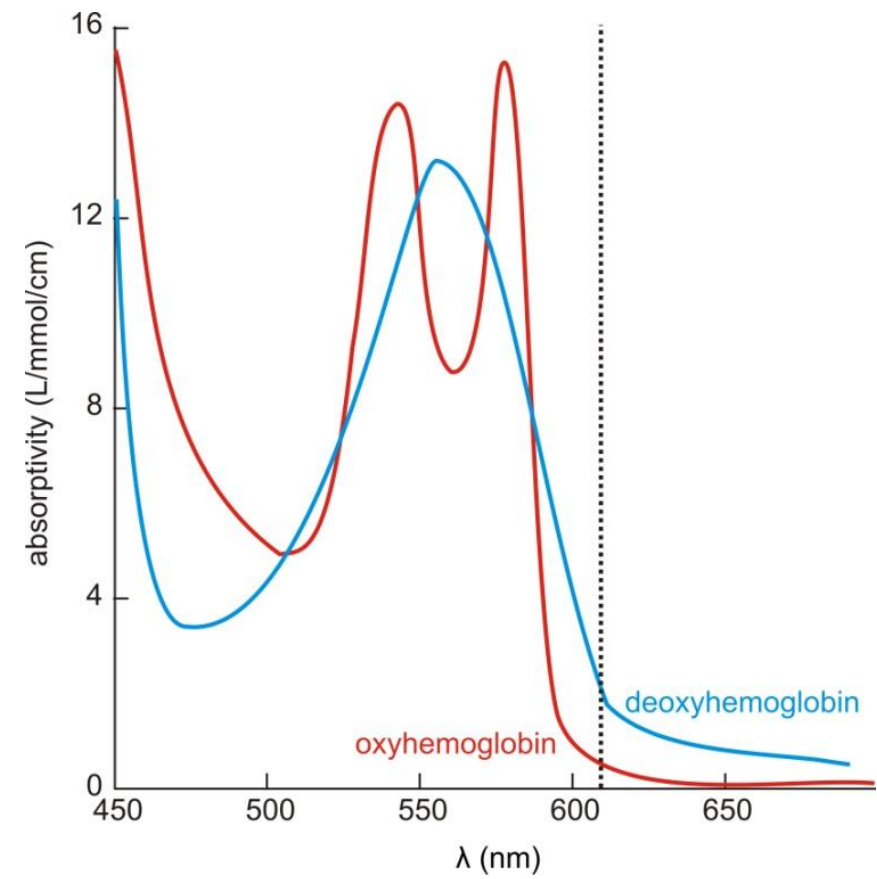

Figure 14: Absorption spectra of deoxy- and oxyhemoglobin. Due to the different absorptions and therefore reflections of oxy- and deoxyhemoglobin, differences in the oxygenation of the blood can be measured. At the wavelength of $610 \mathrm{~nm}$ (dotted line), which is used for optical imaging, deoxyhemoglobin has a higher absorption and therefore lower reflectance than oxyhemoglobin. Modified from Hallum et al. (2006).

Another main component of the imaging signal originates from a blood volume increase, supplying highly oxygenated blood to the activated cortical area. The third component of the intrinsic signal derives from light-scattering changes that go along with cortical activation caused by ion and water movement, capillary expansion, or neurotransmitter release.

This change in reflection, which correlates with the neuronal activity, can be measured in the visual cortex. To do so, a temporally periodic stimulus was continuously presented to the animal and the cortical response at the stimulus frequency was extracted by Fourier analysis. Like that the stimulus-induced signal could be clearly extracted from the heartbeat-, respiration-, and vasomotor-artifacts. Optical images of cortical intrinsic signals were acquired by using a Dalsa 1M30 CCD camera (Dalsa, Waterloo, Canada) with a 50 x $50 \mathrm{~mm}$ tandem lens configuration (Nikon, Tokyo, Japan), which allowed the simultaneous recording of visual cortex activities of both hemispheres. The recorded area had a size of $12.3 \times 12.3 \mathrm{~mm}^{2}$ (Figure 15). In the EE experiments in the PD 130 group a $135 \times 50 \mathrm{~mm}$ tandem lens configuration for just one hemisphere was used. For more detailed information on pixel and images sizes see Table 3 . 


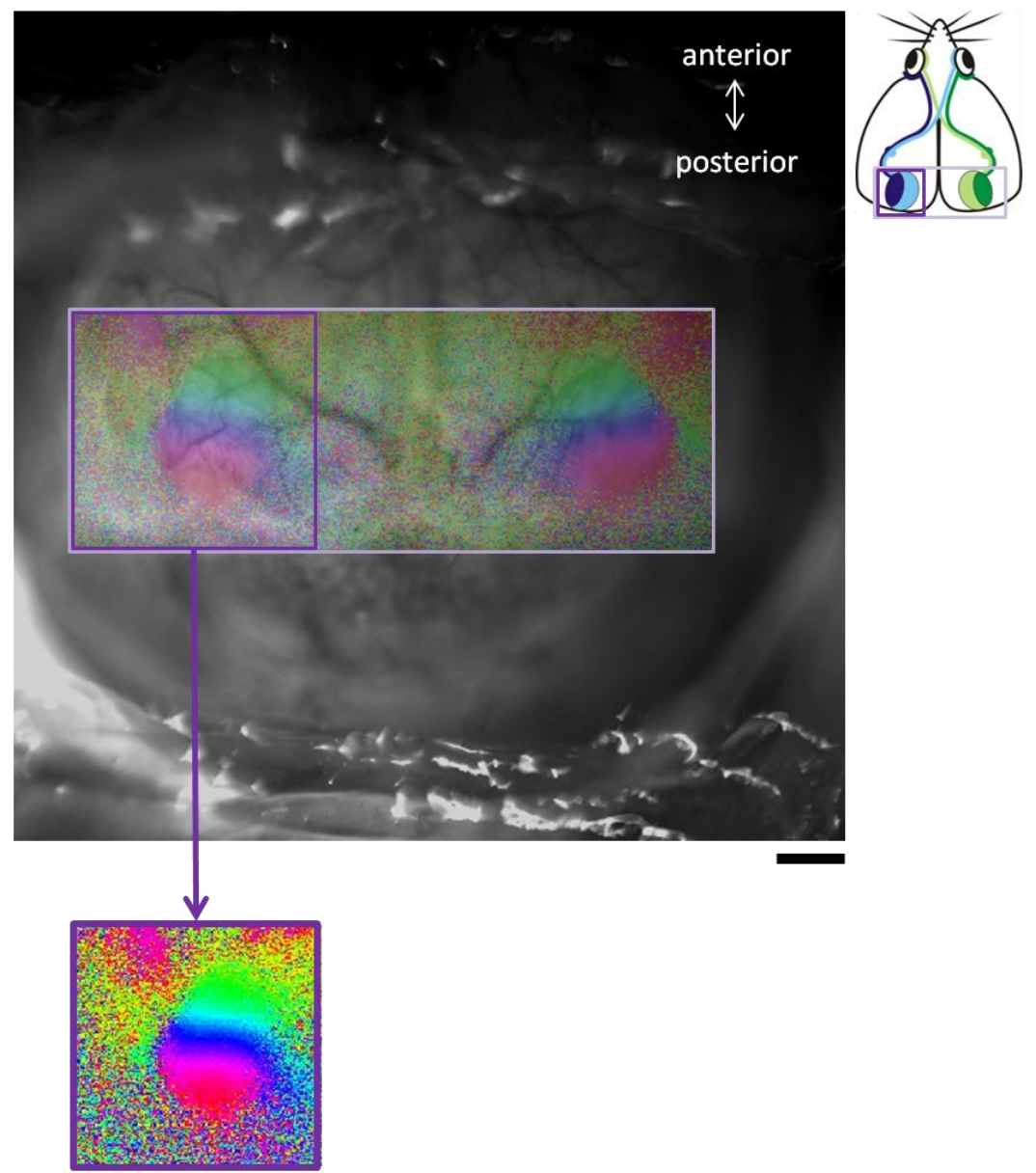

Figure 15: Visualization of the recorded area of the mouse brain with a $50 \times 50 \mathrm{~mm}$ lens. View from above. In the back a black-and-white picture of the vascular pattern is shown (recorded area: $12.3 \times 12.3 \mathrm{~mm}^{2}$ ). A phase map after simultaneous stimulation of both eyes is overlaid. For the analysis of the visual cortex of both hemispheres $3.6 \times 9.6 \mathrm{~mm}$ pictures are cropped (light purple frame, see also inset). For the analysis of just one hemisphere, in this example the left hemisphere, a $3.6 \times 3.6 \mathrm{~mm}$ picture is cropped (purple frame). Scale bar: $1 \mathrm{~mm}$.

Table 3: Size of the images in number of pixels and millimeter $(\mathbf{m m})$ before and after cropping, depending on the region to be analyzed and the used lenses. In the described experiments mainly the $50 \times 50 \mathrm{~mm}$ lenses were used to acquire data of the visual cortex in both hemispheres.

\begin{tabular}{|c|c|c|c|c|}
\hline Lenses & Pixel size & Acquired image & $\begin{array}{l}\text { Cropped image } \\
2 \text { hemispheres }\end{array}$ & $\begin{array}{l}\text { Cropped image } \\
1 \text { hemisphere }\end{array}$ \\
\hline $50 \times 50 \mathrm{~mm}$ & $24 \times 24 \mu \mathrm{m}$ & $\begin{array}{l}512 \times 512 \text { pixels } \\
12.3 \times 12.3 \mathrm{~mm}\end{array}$ & $\begin{array}{c}150 \text { x } 400 \text { pixels } \\
3.6 \text { x } 9.6 \mathrm{~mm}\end{array}$ & $\begin{array}{c}150 \times 150 \text { pixels } \\
3.6 \times 3.6 \mathrm{~mm}\end{array}$ \\
\hline $135 \times 50 \mathrm{~mm}$ & $8.889 \times 8.889 \mu \mathrm{m}$ & $\begin{array}{c}512 \text { x } 512 \text { pixels } \\
4.6 \text { x } 4.6 \mathrm{~mm}\end{array}$ & & $\begin{array}{l}300 \times 300 \text { pixels } \\
2.67 \times 2.67 \mathrm{~mm}\end{array}$ \\
\hline
\end{tabular}

The surface vascular pattern and intrinsic signal images were visualized with illumination wavelengths set by a green $(550 \pm 10 \mathrm{~nm})$ or red $(610 \pm 10 \mathrm{~nm})$ interference filter, respectively. After acquisition of a surface image, the camera was focused $600 \mu \mathrm{m}$ below the 
cortical surface. An additional red light filter was interposed between the brain and the camera to shield it from scattered light. Frames were acquired at a rate of $30 \mathrm{~Hz}$ temporally binned to $7.5 \mathrm{~Hz}$ and stored as $512 \times 512$ pixels images after spatial binning (without binning: $1024 \times 1024$ pixels) of the camera image. A high refresh rate stimulus monitor (for PT experiments and PD 130 group of EE experiments: D2128-TCO, Dell, Austin, Texas; $40 \times 30 \mathrm{~cm} \triangleq 77^{\circ} \times 58^{\circ}$ in the visual field [length $\mathrm{x}$ height]; for other EE experiments: Hitachi, ACCUVUE, HM-4921-D; $40.6 \times 30.5 \mathrm{~cm} \triangleq 78^{\circ} \times 59^{\circ}$ ) displayed visual stimuli and was placed in front of the mouse, aligned with its midline to the animal and at a distance of $25 \mathrm{~cm}$ (Figure 16).

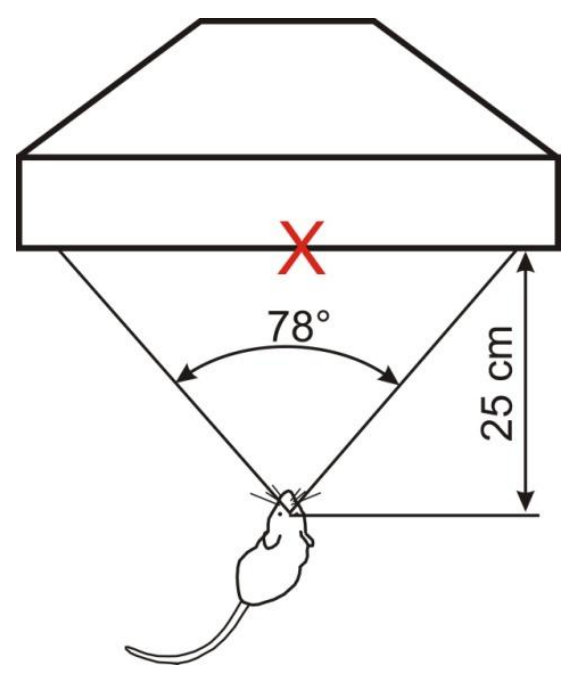

Figure 16: Scheme of the setup for visual stimulation. The midline of the monitor (red cross) was aligned to the midline of the mouse. The monitor is positioned straight in front of the mouse. Modified from Kalatsky \& Stryker (2003).

On the stimulus monitor a drifting white horizontal bar $\left(2^{\circ}\right.$ wide, corresponding to a spatial frequency of $0.25 \mathrm{cyc} / \mathrm{deg}$ ) on black background was generated by the program ContStim (CONTinousSTIMulation; VK Imaging, Houston, Texas) by a Matrox G450 board (Matrox Graphics, Inc., Quebec, Canada; for PT experiments and PD 130 group of EE experiments) or NVIDIA GeForce gt250 (for other EE experiments) graphics board, respectively. The distance between two bars in the visual field was $70^{\circ}$ and they were presented at a temporal frequency of $0.125 \mathrm{~Hz}$. To obtain activity and retinotopic maps of the visual cortices of both hemispheres simultaneously, both eyes were open and the moving bar was presented over the whole length of the screen, to stimulate both eyes evenly (Figure 17 A). In this way, activity in the left and right hemisphere could be obtained at the same time and could be compared 
between the hemispheres. To assess the OD of the recorded hemisphere the stimulus bar was restricted to the binocular visual field of $\mathrm{V} 1$ of the recorded hemisphere $\left(-5^{\circ}\right.$ to $+15^{\circ}$ azimuth for the left and $-15^{\circ}$ to $+5^{\circ}$ azimuth for the right visual cortex) (Figure 18) and animals were stimulated through either the left or the right eye in alternation while the other one was covered by aluminum foil (Figure $17 \mathrm{~B}-\mathrm{E}$ ).
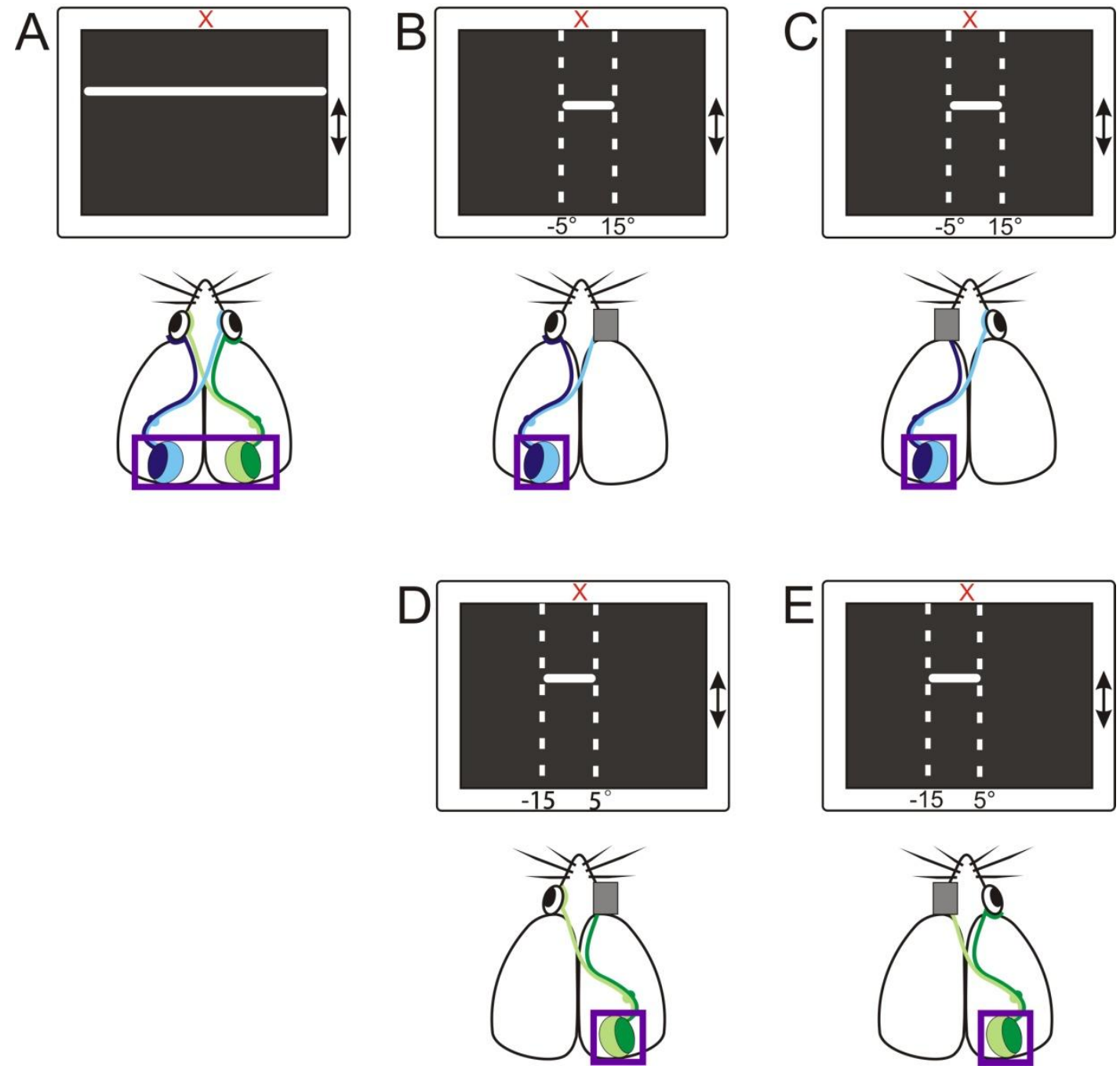

Figure 17: Visual stimuli used. Position of the stimulus bar and stimulated eyes during the different stimulus conditions, respectively. The red cross marks the midline of the screen and the purple boxes the analyzed hemispheres. (A) Setup for the simultaneous stimulation of both eyes. Bar length over the whole screen. Both eyes open. Analysis of both hemispheres. (B and C) Setup for the monocular stimulation of the binocular region of the left visual cortex. After each run the respective other eye was covered with aluminum foil. Length of bar $20^{\circ}$ from $-5^{\circ}$ to $+15^{\circ}$ in the visual field. Analysis of left hemisphere. (D and E) Setup for the monocular stimulation of the binocular zone of the right visual cortex. Length of bar $20^{\circ}$ from $-15^{\circ}$ to $+5^{\circ}$ in the visual field. Analysis of right hemisphere. 


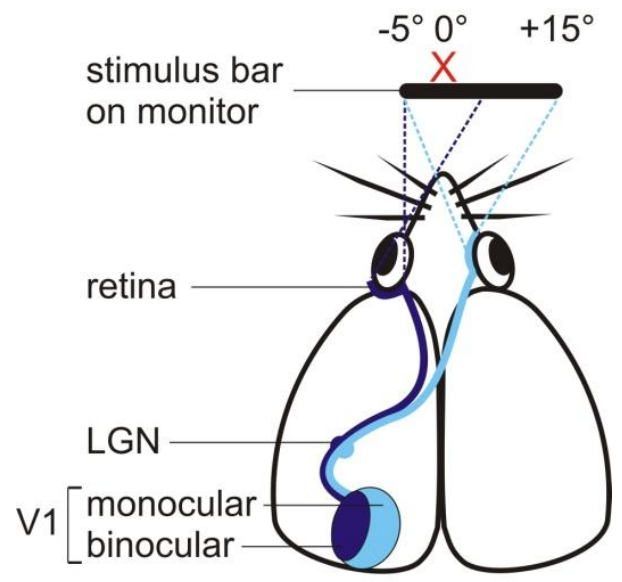

Figure 18: Stimulation of the binocular zone in V1 of the left hemisphere. The stimulus bar on the stimulus monitor had a length of $20^{\circ}$ in the visual field of the mouse. The center of the bar was shifted $5^{\circ}$ to the right in correspondance to the center of the stimulus monitor (marked by the red cross) (from $-5^{\circ}$ to $+15^{\circ}$ in the visual field ) in order to stimulate the binocular cortex of the left hemisphere.

A hemodynamic delay is due to the delay in the change in the absorption of the blood. To correct for that in the map calculation, the stimuli were presented in two opponent moving directions. First, the stimulus was presented moving upwards and in the next stimulus setting moving downward.

Visual cortical maps were calculated from the acquired frames by Fourier analysis to extract the signal at the stimulation frequency using "iman" (IMage ANalysis; VK Imaging, Houston, Texas), then the area of the visual cortex was cropped with "mapans" (MAP ANalysis Single; VK Imaging, Houston, Texas). Depending on whether one or two hemispheres were analyzed and which lenses were used, the size of the image (number of pixels) was chosen accordingly (Table 3, Figure 15).

Next, the activity signal was normalized with respect to the background by shifting every pixel of the map by the vector that is the average of all pixels in the selected background region. Maps which were acquired with the stimulus bar moving upwards or downwards respectively were combined by "mapanm” (MAP ANalysis Multiple; VK Imaging, Houston, Texas) to an average map (for an example of average maps see Figure $19 \mathrm{C}-\mathrm{E}$ ). Thereby the hemodynamic delay could be corrected. The phase component of the signal is used for the calculation of retinotopy. The phase map color-codes the activated area in the visual cortex by referring to a position of the stimulus bar on the monitor at that moment (Figure $19 \mathrm{~A}-\mathrm{C}$ ). The amplitude component of the signal represents the intensity of neuronal activation (expressed as fractional change in reflectance $\times 10^{4}$ ) (Figure 19 D) and was used to calculate 
OD and the hemisphere activation quotient. The polar map combines the information of both maps: retinotopy and activity are overlaid (Figure $19 \mathrm{E}$ ).
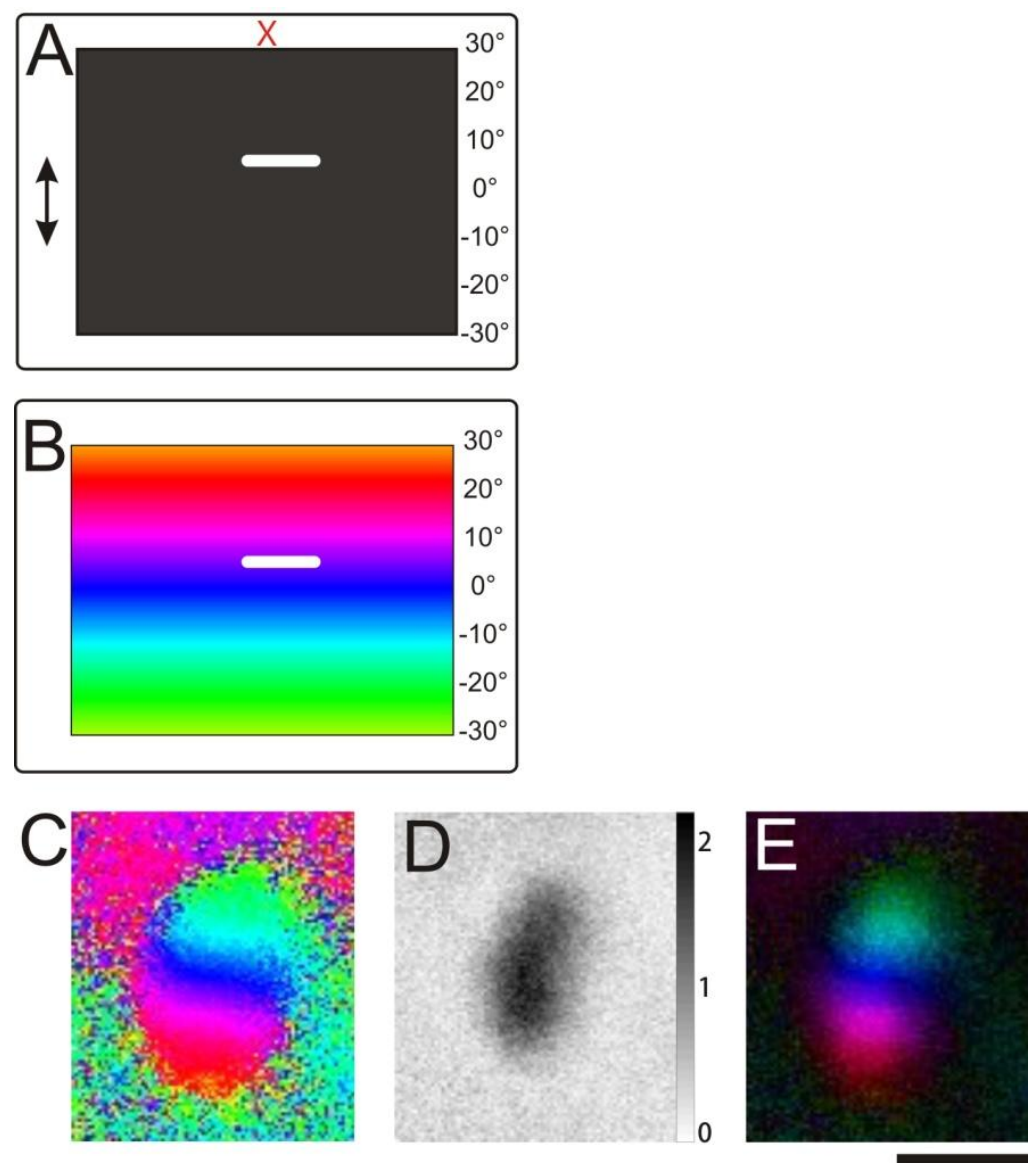

Figure 19: Color-coded phase map and gray-scale coded magnitude map. (A) An upwards or downwards moving white bar on black background is shown on a stimulus monitor to provoke activity in the visual cortex. The degrees on the edge of the monitor indicate the position of the bar relative to the center of the monitor $\left(0^{\circ}\right)$. In this example, the bar is located about $5^{\circ}$ above the center. The red cross marks the midline of the stimulus monitor. (B) The color code visualizes the cortical responses to different locations of the bar. The cortical response to the stimulus shown in (B) would be coded as blue. (C) In the retinotopic phase map acquired by optical imaging (here: image of the left visual cortex during stimulation of the binocular zone through the contralateral right eye) the color code can be retrieved. The different positions of the bar can be found again in the color matched activated areas in the visual cortex. The retinotopy can be recognized. Neighbored colors in the color code of the bar position are also neighbored in the retinotopic phase map. (D) Activity map of the same experiment. Darker areas code for stronger activation. (E) Polar map of the same experiment. The polar map combines the retinotopic map with the activity map. Higher activity is encoded by lighter areas. Scale bar: $1 \mathrm{~mm}$.

The strength of activation in the two hemispheres was compared by calculating a hemisphere activation quotient as the average of neuronal activation of the right hemisphere divided by the average activation of the left hemisphere (activation ${ }_{\text {right }}$ activation $_{\text {left }}$ ) after binocular 
stimulation with a moving horizontal bar extending over $77^{\circ}$ azimuth of the visual field. A quotient of 1 indicates that both hemispheres were activated equally strong; a quotient less than 1 represents stronger activation of the left hemisphere; a quotient higher than 1 indicates stronger activation of the non-lesioned (right) hemisphere. Hemisphere activation quotients were calculated from maps that had at least a response magnitude of $1.3 \times 10^{-4}$ and recognizable retinotopy. At least 3 hemisphere activation quotients were obtained per animal and then used for calculating an average quotient.

To assess the OD of the recorded hemisphere, the bar was exclusively shown in the binocular zone of the visual cortex to be analyzed as described before. The cropped and normalized average maps were used to calculate the ODI as developed by Cang et al. (2005) using MATLAB (R2011a). First, the ipsilateral eye magnitude map was smoothed to reduce pixel shot noise by low-pass filtering using a uniform kernel of $5 \times 5$ pixels. The smoothed map was then thresholded at $30 \%$ of peak response amplitude to eliminate the background noise and to delineate the region that produced the strongest response to the ipsilateral eye. After this process, the value of the pixel with the strongest response was determined as the maximum response of the ipsi- or contralateral eye, respectively. Then the OD score for every pixel in this region was computed as:

$$
O D I=\frac{(C-I)}{(C+I)}
$$

with $\mathrm{C}$ and I representing the raw response magnitudes of each pixel to visual stimulation of the contralateral and ipsilateral eye, respectively. Then an OD-index (ODI) was computed as the average of the OD-scores of all responsive pixels. Cells within the binocular zone of the visual cortex are differently activated by stimulation of both eyes (Hubel \& Wiesel 1962). The ODI describes the ratio of the cortical activity induced by contralateral eye stimulation compared to ipsilateral eye stimulation. Therefore the ODI is a measure for eye dominance and is used for measuring OD-plasticity. The ODI can range from -1 to +1 , with negative values representing ipsilateral and positive values representing contralateral dominance (Cang et al. 2005). An ODI of 0 would indicate an equal activation of pixels by contra- and ipsilateral eye stimulation. We calculated ODIs from blocks of 4 runs in which the averaged maps for each eye had at least a response magnitude of $1 \times 10^{-4}$. We obtained at least 3 ODIs per animal (for each hemisphere). All ODIs of one animal were averaged for further quantification and data display. The ODIs were color-coded in a two-dimensional map of the OD-scores (OD-map): cold blue colors represent negative values (ipsilateral eye dominance) 
and warm red colors represent positive values (contralateral eye dominance). An example for the ODI assessment and an OD-shift in the left hemisphere is shown in Figure 20.

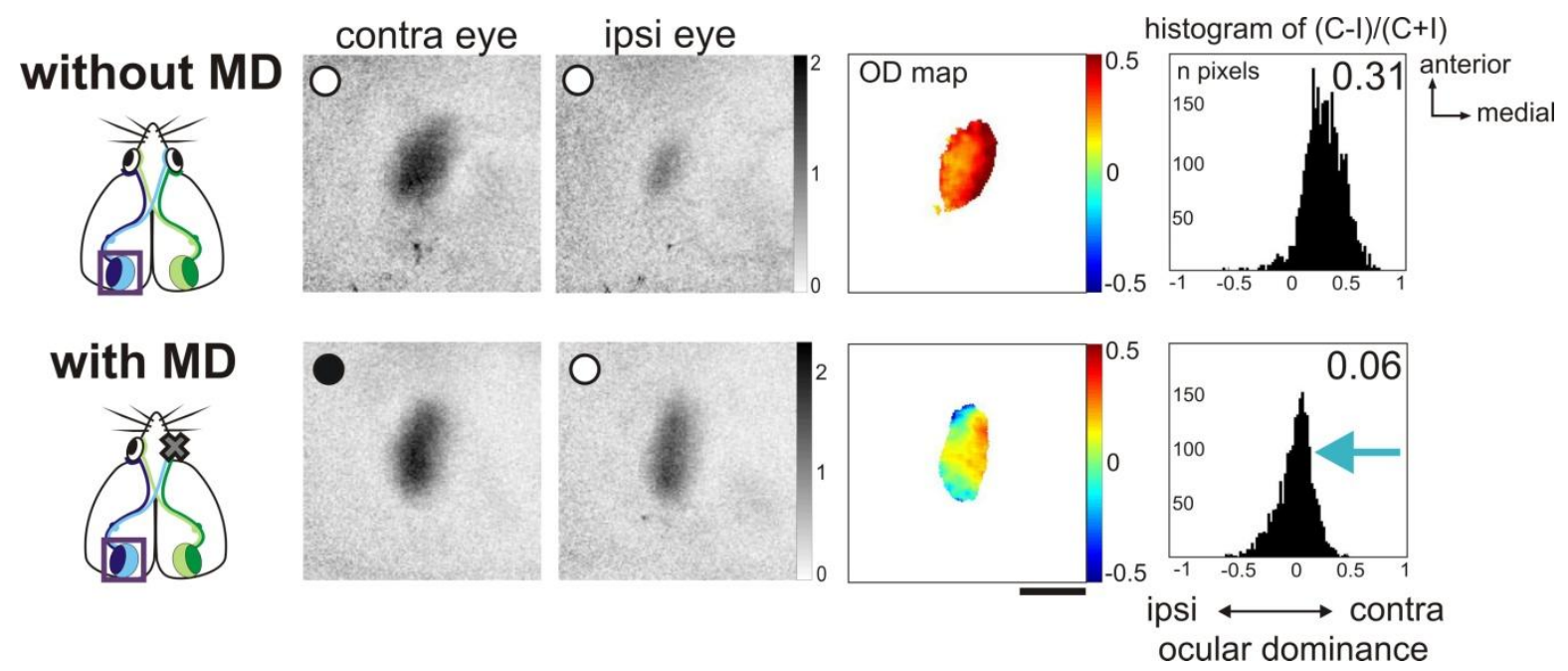

Figure 20: ODI and OD-shift. Optically imaged gray-scale coded response magnitude maps in the binocular region of mouse visual cortex and their ODI quantification in control animals without MD (top) and with MD (bottom). The magnitude of the optical responses is illustrated as fractional change in reflection $\times 10^{4}$. Without MD, activity patches evoked by stimulation of the contralateral eye were darker than those after stimulation of the ipsilateral eye. This indicates contralateral dominance. Unfilled circles in the top left corner of the images represent the two open eyes. In the twodimensional OD-maps ODI values are color-coded: red, warm colors represent a positive OD. The OD-scores in the histogram mainly reside in the positive values, as also indicated by the positive mean ODI of this animal in the top right corner. A MD induced a significant OD-shift toward the open eye: the response magnitude maps of both contra- (deprived eye, black filled circle) and ipsilateral (open eye, unfilled circle) eye were equally dark, colder colors which encode for lower OD-values prevailed in the OD-map, the histogram of OD-scores shifted to the left and the ODI was 0.06 compared to 0.31 without MD. Scale bar: $1 \mathrm{~mm}$.

\subsection{Electrophysiology}

As a technique which does not rely on changes in the blood flow/oxygenation we additionally performed extracellular electrophysiological recordings. Experiments were performed by Dr. Klaus Kreikemeier at the University of Jena (Greifzu et al. 2011). The recordings were obtained from the binocular field of V1 (as determined by intrinsic signal optical imaging) of 4 control (sham) and 5 PT-mice after 7 days of MD. The animals were between 96 and 99 days of age at the time of electrophysiological recordings. To access binocular V1, a $5 \times 5 \mathrm{~mm}$ craniotomy performed over the left hemisphere, contralateral to the deprived eye. Warm agarose $\left(2.5 \%\right.$ in $\left.0.9 \% \mathrm{NaCl}, 37^{\circ} \mathrm{C}\right)$ was placed over the exposed area to protect the cortex and reduce brain pulsations. Glass-coated tungsten microelectrodes (FHC) with tip 
resistances of 0.8 to $1 \mathrm{M} \Omega$ were used to record multiunit activity. Electrodes were positioned at a depth of 280 to $610 \mu \mathrm{m}$ below the pial surface (layers II/III and IV), spaced evenly across the binocular region of V1. Signals were acquired using a SUA-filter amplifier (Thomas Recording) and analyzed with Spike 2 (CED). For multiunit recording, the extracellular signal was filtered from 0.5 to $10 \mathrm{kHz}$ and sampled at $25 \mathrm{kHz}$ (Micro 1401; CED). Using a template building algorithm implemented in the acquisition system (Spike2; CED), we separated single units ( $1.4 \mathrm{~ms}$ time window, sampling frequency $25 \mathrm{kHz}$ corresponding to 35 data points) from our multiunit recordings. At all recording sites, only the single unit eliciting the highest amplitude spikes entered further quantitative analyses. A moving bar (length of $4^{\circ}$, temporal frequency of $0.25 \mathrm{~Hz})$ restricted to the binocular field of the left hemisphere $\left(-5^{\circ}\right.$ to $+15^{\circ}$ azimuth) was used as a visual stimulus. Optimal stimuli were presented to either eye alternately and the relative strength of the single-unit responses was determined. Neurons were assigned an OD score according to the 1 to 7 scale of Hubel and Wiesel (1962). In addition, we calculated a contralateral bias index (CBI) (Gordon \& Stryker 1996) according to the formula: $\mathrm{CBI}=\left[\left(\mathrm{n}_{1}-\mathrm{n}_{7}\right)+(2 / 3)\left(\mathrm{n}_{2}-\mathrm{n}_{6}\right)+(1 / 3)\left(\mathrm{n}_{3}-\mathrm{n}_{5}\right)+\mathrm{N}\right] / 2 \mathrm{~N}$ where $\mathrm{N}=$ total number of cells and $\mathrm{n}_{\mathrm{x}}=$ number of cells with OD scores equal to $\mathrm{x}$.

\subsection{Perfusion and tissue processing}

Directly after the optical imaging experiment, mice were deeply anesthetized with $30 \%$ chloral hydrate $(0.2 \mathrm{ml}$, intraperitoneal injection) and perfused transcardially with $1 \%$ heparin in $0.9 \% \mathrm{NaCl}$ for 2 minutes and $4 \%$ paraformaldehyde (PFA) ( $\mathrm{pH} 7.4$ ) for 3 min. The brain was removed and postfixed in $4 \%$ PFA ( $\mathrm{pH}$ 7.4) for one day and then transferred to cryoprotectant solution (10\% sucrose, $20 \%$ glycerol). The brains were frozen in methylbutan and stored at $-80^{\circ} \mathrm{C}$. Before slicing, a lateral-ventral cut was positioned on the right side of the brain over the whole anterior to posterior extend of the brain in order to distinguish between right and left hemisphere after cutting and staining. Coronal brain sections were cut on a sledge microtome at $40 \mu \mathrm{m}$ (Leica SM 2010R), collected and stored free floating in antifreeze solution (30\% ethylene glycol, $15 \%$ glucose in phosphate buffer (PB)) at $-20{ }^{\circ} \mathrm{C}$.

In PT-experiments (except for EE), the protocol was slightly different. Mice were perfused

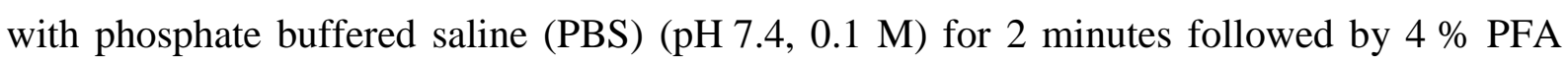
(pH 7.4) for 8 minutes. The brain was cryoprotected with a $10 \%$ sucrose solution in PBS for one day followed by a $30 \%$ sucrose solution in PBS for 1 or 2 days. 


\subsection{Nissl staining}

Brain sections were mounted on microscope slides and Nissl-stained for analysis of the PTlesion. First sections were dehydrated in an ascending ethanol series: 70 \%, $80 \%, 96 \%$, $100 \%$ ethanol for 2 minutes each. Then they were rehydrated by shortly dipping them into bidest. water and stained in cresyl violet $(0.5 \%)$ for about 2 minutes. De-staining and rehydration was carried out in $70 \%, 80 \%, 96 \%, 100 \%$ ethanol for 2 minutes each. For faster destaining, acetic acid was supplemented to the $70 \%$ solution. Sections were transferred to xylol and coversliped with entellan. Cresyl violet solution was kindly provided by Patricia Sprysch (research group Prof. Staiger, Neuroanatomy, Göttingen).

To determine size and location of the cortical lesions, every third section (for non-EE experiments every $6^{\text {th }}$ section) was analyzed with a microscope (Axioskop 50, Carl Zeiss; 20x) and lesion size was measured using AxioVision (AxioVs 40 4.5.0.0./4.8.2.0). Animals in which the lesion extended into the white matter or V1 were excluded from further analyses.

\subsection{Immunohistochemistry}

The number of PNNs and of PV cells was examined in mice raised in SC or EE cages, respectively, in 4 mice per group. Mice without MD from the SC-group PD 235 and EEgroup PD 220 were used. PNNs were labeled cytochemically by N-acetylgalactosaminebinding (in the glycosaminoglycan chains of the chondroitinsulfat-proteoglycanes) by Wisteria floribunda agglutinin (WFA), an established marker for PNNs (Hartig et al. 1992). Coronal brain sections $(40 \mu \mathrm{m})$ that included $\mathrm{V} 1(\approx 2.70 \mathrm{~mm}$ to $3.88 \mathrm{~mm}$ posterior to bregma) were used, out of which 4 sections (approximately every $300 \mu \mathrm{m}$ ) per mouse were analyzed. Free floating sections were washed with $0.1 \mathrm{M}$ PB and incubated for 30 minutes in a blocking solution (10\% donkey serum, $0.3 \%$ Triton $\mathrm{X}-100$ in $\mathrm{PB}, \mathrm{pH} 7.4)$ at room temperature. Afterwards, the brain sections were washed with $0.1 \mathrm{M}$ PB. Subsequently, sections were incubated overnight at $4{ }^{\circ} \mathrm{C}$ with mouse anti-PV (Immunological Science, 1:500) and biotinconjugated lectin WFA (Sigma, 1:1000) in $0.1 \mathrm{M}$ PB including $0.3 \%$ Triton X-100. After washing the sections again in $0.1 \mathrm{M} \mathrm{PB}$ antibodies were revealed with $\mathrm{Cy} 2$-conjugeated donkey anti-mouse (Biotium, 1:200) and Cy3-conjugated streptavidin (Jackson ImmunoResearch, 1:1000) in 0.1 M PB including 0.3\% Triton X-100 (2 hours incubation at room temperature, light protected). Sections were washed again in $0.1 \mathrm{M}$ PB before they were transferred on a microscope slide and dried for 30 minutes. Finally, the dried sections were 
mounted with Fluoromount and a glass cover slip was placed over the area. The mounting medium included 4'.6-diamidin-2-phenylindol (DAPI) to stain and visualize all cell nuclei. After drying overnight at $4{ }^{\circ} \mathrm{C}$ slides were stored in a light tight box at $4{ }^{\circ} \mathrm{C}$.

Pictures of $\mathrm{V} 1$ in the stained sections were taken with a fluorescence microscope (Axio Imager.M2, Zeiss) with the help of Robin Wagener in the laboratory of Prof. Staiger (Neuroanatomy, University Göttingen). The V1 area was identified by its high number of PV and WFA-positive cells (see Figure $21 \mathrm{~A}$ ), the selected area was verified by comparison with a mouse brain atlas (Paxinos \& Franklin 2001) (Figure 22). Exposure time for the acquisition was optimized for each picture. Images of V1 (Figure 21 B) of each hemisphere were acquired using Neurolucida (version 10.51, MBF Bioscience) under a 25x objective, using the following filters: DAPI (Zeiss 49) excitation: 335-383 nm, emission: 420-470 nm; FITC (Cy2, Zeiss 44): excitation: 455-495 nm, emission: 505-555 nm; DSred (Cy3, Zeiss43) excitation: 533-558 nm, emission: 570-640 nm. A 3-dimensional tissue stack (3D volume in $\mathrm{x}, \mathrm{y}$ and $\mathrm{z}$ axis) of the whole V1 area within one section was taken. On average one virtual tissue consisted of 8 tiles ( $4 \mathrm{x}, 2 \mathrm{y}$, size of one tile: $355 \times 270 \mu \mathrm{m}$ ) with 12 to 14 focal planes with a distance of $3 \mu \mathrm{m}$ spanning the full thickness $(40 \mu \mathrm{m})$ of the tissue. PV-positive labeled and WFA-positive labeled cells in layers II-VI of V1 in both hemispheres were counted in the acquired images using Fiji (Schindelin et al. 2012), which is a distribution of the open-source software ImageJ (Rasband). The area in which the cells were counted was also measured with Fiji and the number of cells was interpolated for a volume of one $\mathrm{mm}^{3}$. A mean value of each animal from all its' counted sections was calculated and used for statistics and mean values of the groups. 

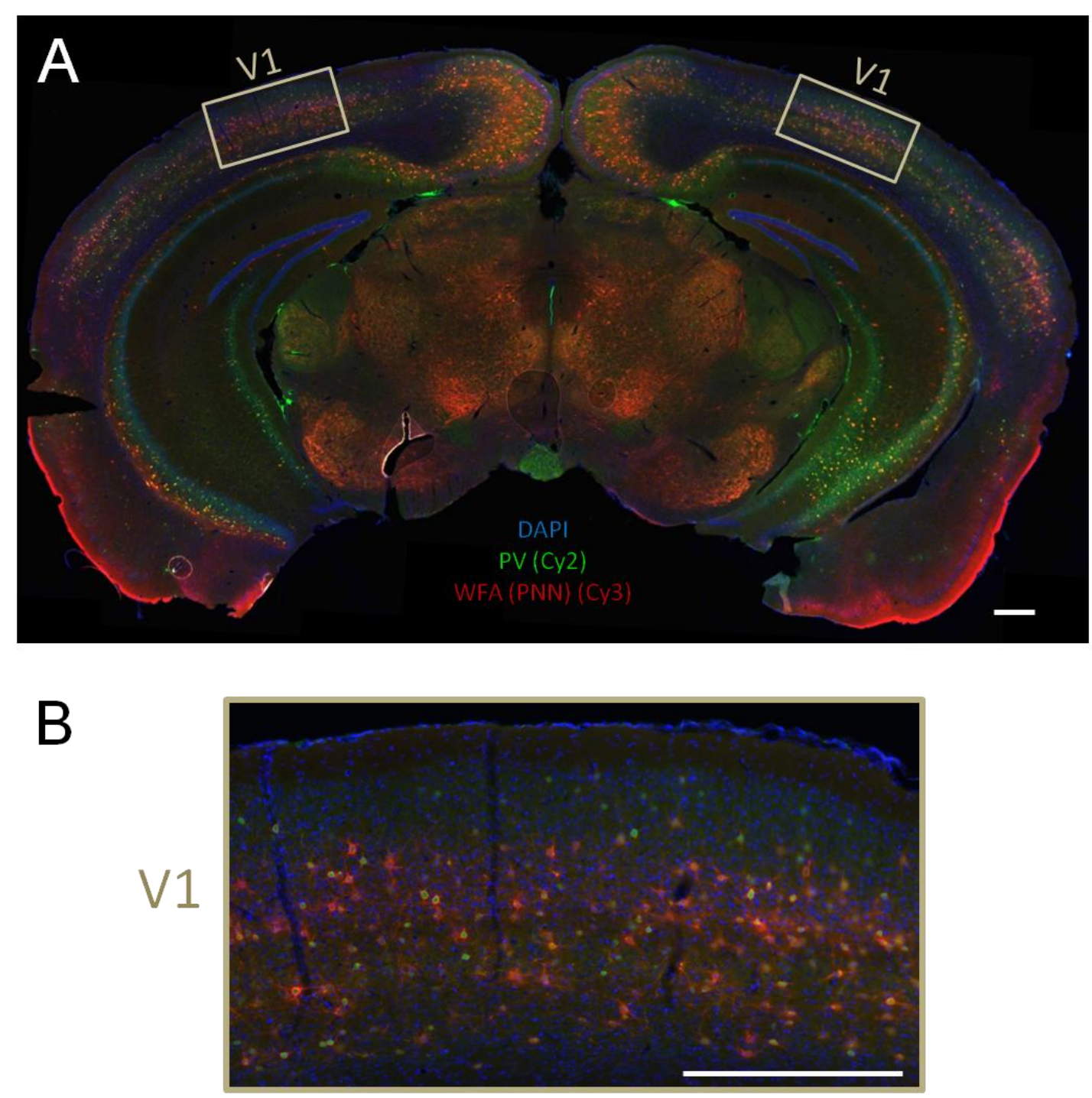

Figure 21: Areas that were analyzed for the PV-positive cells and PNNs in V1. Shown is a frontal brain section of a mouse with fluorescent staining of cell nuclei (DAPI) in blue, PV-positive cells in green (Cy2) and WFA-positive PNNs (Cy3) in red. (A) The white squares mark V1 in which layers IIVI were analyzed. One picture of each hemisphere was taken. This picture was taken as tiles with a 10x objective and assembled to one overview picture by Neurolucida software. (B) Enlargement of V1 of the picture above. Scale bars in A and B: $500 \mu \mathrm{m}$. 


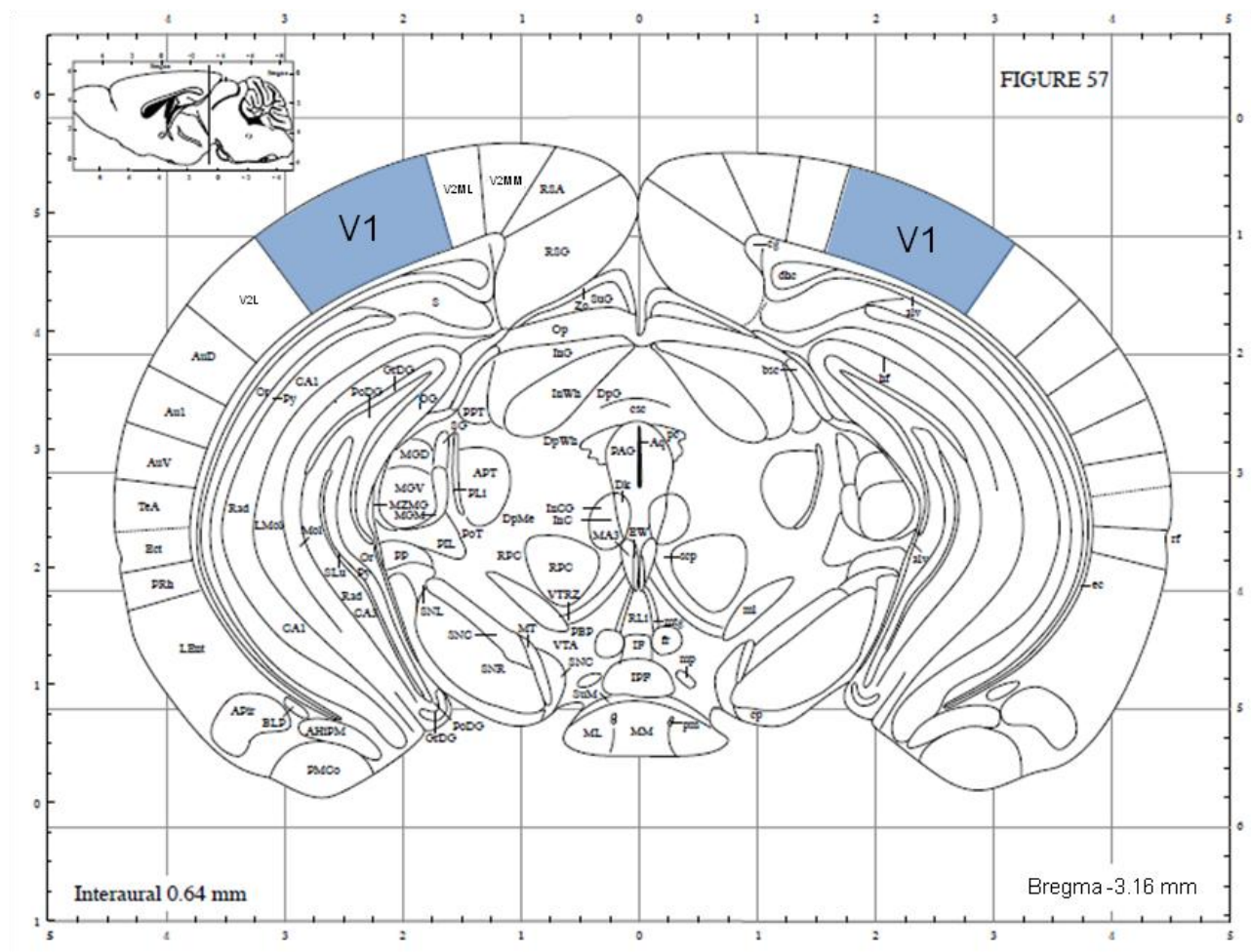

Figure 22: Frontal mouse brain section scheme from Paxinos mouse brain atlas corresponding to the stained section shown in Figure 21. V1, which was the analyzed region, is highlighted. Modified from Paxinos (2001).

\subsection{Statistical analyses}

Statistical analyses were done using Microsoft Excel 2010 and SPSS (20.0.0). Graphs were prepared with GraphPad Prism (version 5.04). All inter- and intragroup comparisons were done by a two-tailed Student t-test with Bonferroni correction. The intergroup comparison of the enhancement of visual acuity was analyzed by ANOVA with repeated measurements with Bonferroni correction. Distributions of electrophysiological data were analyzed with a $\chi^{2}$ test. The levels of significance were set as $* \mathrm{P}<0.05$; $* * \mathrm{P}<0.01 ; * * * \mathrm{P}<0.001$. Data are represented as means \pm SEM. 


\subsection{Used chemicals, antibodies and solutions}

Chemicals, drugs and substances

$\mathrm{PZN}=$ pharmaceutical identification number (,Pharmazentralnummer")

\begin{tabular}{|c|c|c|}
\hline Chemical/drug & Company & Order number/PZN \\
\hline $0.9 \% \mathrm{NaCl}$ & Braun & PZN: 2946431 \\
\hline 2-Methylbutan & Roth & 3927.1 \\
\hline Acetic acid & Roth & 3738.1 \\
\hline Agarose & Biomol & 1280 \\
\hline Atropine & Franz Köhler & PZN: 1821288 \\
\hline Braunovidon gel & Braun & PZN: 2336939 \\
\hline Chloral hydrate & Sigma & 15307 \\
\hline Chlorprothixene & Sigma & C1671 \\
\hline Corneregel Fluid & Dr. Gerhard Mann GmbH & PZN: 6343617 \\
\hline Corneregel eye gel & Dr. Gerhard Mann GmbH & PZN: 1224641 \\
\hline Cresyl violet & Merck & 5235 \\
\hline Dexa-ratiopharm (dexamethasone) & Ratiopharm & PZN: 7720996 \\
\hline Diazepam & Rotexmedica & PZN: 3731425 \\
\hline Entellan & Merck & 107961 \\
\hline Ethanol (99.8 \%) & Roth & K928.1 \\
\hline Ethylene glycol & Fluka & 03750 \\
\hline Fluormont+DAPI & Southern Biotech & 0100-20 \\
\hline Gentamycin gel & Medphano & PZN: 6877307 \\
\hline Glucose & Roth & 6780.1 \\
\hline Glycerol & Roth & 3783.1 \\
\hline Halothane & Sigma & B4388 \\
\hline Heparin & Rotexmedica & PZN: 3862340 \\
\hline Ibuprofen & Fluka & I1892 \\
\hline Isoflurane (Forene) & Abbott & PZN: 4831850 \\
\hline $\mathrm{Na}_{2} \mathrm{HPO}_{4}$ & Roth & P030.2 \\
\hline $\mathrm{Na}_{2} \mathrm{HPO}_{4} \times 2 \mathrm{H}_{2} \mathrm{O}$ & Roth & 4984.1 \\
\hline $\mathrm{NaCl}$ & Roth & 3957.1 \\
\hline $\mathrm{NaH}_{2} \mathrm{PO}_{4} \times \mathrm{H}_{2} \mathrm{O}$ & Roth & T879.2 \\
\hline $\mathrm{NaH}_{2} \mathrm{PO}_{4} \times \mathrm{H}_{2} \mathrm{O}$ & Roth & K300.2 \\
\hline $\mathrm{NaOH}$ & VWR & 28.244 .262 \\
\hline
\end{tabular}




\begin{tabular}{|c|c|c|}
\hline Narcoren (Pentobarbital) & Merial & 3580 \\
\hline PFA & Roth & 0335.3 \\
\hline Rimadyl (Carprofen) & Pfizer & PZN: 0110208 \\
\hline Rose bengal & Sigma & R3877 \\
\hline Sucrose & Roth & 4621.1 \\
\hline Silicon oil & Roth & 4060.1 \\
\hline Sodium acetate & Merck & 6267 \\
\hline Sodium azide & Roth & K305.1 \\
\hline Triton X-100 & Sigma & X100 \\
\hline Xylol & Roth & 4436.1 \\
\hline
\end{tabular}

$\underline{\text { Antibodies and serum }}$

\begin{tabular}{|c|}
\hline Antibody/serum \\
\hline Normal Donkey Serum \\
\hline Mouse anti Parvalbumin \\
\hline biotin-conjugated lectin Wisteria \\
Floribunda agglutinin (WFA) \\
\hline
\end{tabular}

Cy2 Donkey Anti-Mouse

Cy3-Streptavidin

\begin{tabular}{|c|}
\hline Company \\
\hline Jackson ImmunoResearch \\
\hline Immunological Science \\
\hline Sigma \\
\hline
\end{tabular}

Biotium

Jackson ImmunoResearch
Order number 017-000-121

MAB-10233

L1516

CF 488A, 20014

016-160-084 
$\underline{\text { Solutions }}$

\begin{tabular}{|l|c|c|}
\hline \multicolumn{1}{|c|}{ Solution } & Chemical/drug & Amount \\
\hline Agarose (2.5\%) & $-\quad$ Agarose & $2.5 \mathrm{~g}$ \\
\hline & Fill up to $100 \mathrm{ml}$ with $0.9 \% \mathrm{NaCl}$ & \\
\hline
\end{tabular}

Anti-Freeze solution

(30\% ethylene glycol, $15 \%$ glucose in $\mathrm{PB}$ )

Phosphate buffer $\mathrm{pH} 7.4$ :

Stock A (base):

- $\mathrm{Na}_{2} \mathrm{HPO}_{4}$

$7.098 \mathrm{~g}$

Fill up to $400 \mathrm{ml}$ with bidest. water

Stock B (acid):

- $\mathrm{NaH}_{2} \mathrm{PO}_{4} \times \mathrm{H}_{2} \mathrm{O}$

Fill up to $1000 \mathrm{ml}$ with bidest. water

Phosphate buffer:

- Bidest. water

$700 \mathrm{ml}$

- Stock B $17.5 \mathrm{ml}$

Use Stock A to adjust to $\mathrm{pH} 7.4$

Fill up to $1000 \mathrm{ml}$ with bidest. water

$\underline{\text { Anti-Freeze Solution }}$

- Ethylenglycol

$300 \mathrm{ml}$

- Phosphate buffer

$500 \mathrm{ml}$

- Glucose

$150 \mathrm{~g}$

Fill up to $1000 \mathrm{ml}$ with bidest. water

\begin{tabular}{|c|c|c|}
\hline Chloral hydrate (30 \%) & $\begin{array}{l}\text { - Chloral hydrate } \\
\text { Fill up to } 100 \mathrm{ml} \text { with bidest. water }\end{array}$ & $30 \mathrm{~g}$ \\
\hline Chlorprothixene & $\begin{array}{l}\text { - Chlorprothixene } \\
\text { - Bidest. water }\end{array}$ & $\begin{array}{l}4 \mathrm{mg} \\
1 \mathrm{ml}\end{array}$ \\
\hline Cresyl violet & $\begin{array}{l}\text { - Sodium acetate } \\
\text { - Acetic acid } \\
\text { - Cresyl violet } \\
\text { Fill up to } 1000 \mathrm{ml} \text { with distilled water } \\
\text { Mix and let set overnight }\end{array}$ & $\begin{array}{l}5.4 \mathrm{~g} \\
9.6 \mathrm{~g} \\
5.0 \mathrm{~g}\end{array}$ \\
\hline $\begin{array}{l}\text { Cryoprotection solution } \\
\text { (10\% sucrose, } 20 \% \\
\text { glycerol \& } 0.02 \% \text { sodium } \\
\text { azide) }\end{array}$ & $\begin{array}{l}\text { - Sucrose } \\
\text { - Glycerol } \\
\text { - Sodium azide }\end{array}$ & $\begin{array}{l}10 \mathrm{~g} \\
20 \mathrm{ml} \\
0.02 \mathrm{~g}\end{array}$ \\
\hline
\end{tabular}

Fill up to $100 \mathrm{ml}$ with $0.1 \mathrm{M}$ PB 


\begin{tabular}{|c|c|c|}
\hline Diazepam & $\begin{array}{l}\text { - Diazepam }(5 \mathrm{mg} / \mathrm{ml} \text { solution }) \\
\text { Fill up to } 40 \mathrm{ml} \text { with } 0.9 \% \mathrm{NaCl}\end{array}$ & $2 \mathrm{ml}$ \\
\hline Heparin (1\%) in saline & $\begin{array}{l}\text { - Heparin (25.000 I.E.) } \\
\text { - } \mathrm{NaCl} \\
\text { Fill up to } 1000 \mathrm{ml} \text { with bidest. water }\end{array}$ & $\begin{array}{l}53.33 \mathrm{ml} \\
9 \mathrm{~g}\end{array}$ \\
\hline Ibuprofen & $\begin{array}{l}\text { - } \text { Ibuprofen } \\
\text { - } 0.9 \% \mathrm{NaCl}\end{array}$ & $\begin{array}{l}0.075 \mathrm{~g} \\
10 \mathrm{ml}\end{array}$ \\
\hline $\begin{array}{l}\text { PB } \\
\text { (Phosphate buffer, } 0.1 \mathrm{M} \text { ) }\end{array}$ & $\begin{array}{l}\text { - } \mathrm{NaH}_{2} \mathrm{PO}_{4} \times 2 \mathrm{H}_{2} \mathrm{O} \\
\text { - } \mathrm{Na}_{2} \mathrm{HPO}_{4} \\
\text { Fill up to } 1000 \mathrm{ml} \text { with bidest. water }\end{array}$ & $\begin{array}{l}3.75 \mathrm{~g} \\
9.75 \mathrm{~g}\end{array}$ \\
\hline $\begin{array}{l}\text { PBS } \\
\text { (Phosphate buffered saline) }\end{array}$ & $\begin{array}{l}\text { - } \mathrm{NaCl} \\
\text { Fill up to } 1000 \mathrm{ml} \text { with } \mathrm{PB}\end{array}$ & $9 \mathrm{~g}$ \\
\hline Pentobarbital solution & $\begin{array}{l}\text { - Narcoren }(160 \mathrm{mg} / \mathrm{ml}) \\
\text { Fill up to } 10 \mathrm{ml} \text { with } 0.9 \% \mathrm{NaCl}\end{array}$ & $0.315 \mathrm{ml}$ \\
\hline PFA $(4 \%)$ & & \\
\hline $0.2 \mathrm{M}$ Phosphate buffer & & \\
\hline Stock A (base): & $\begin{array}{l}\text { - } \mathrm{Na}_{2} \mathrm{HPO}_{4} \cdot \text { x } 2 \mathrm{H}_{2} \mathrm{O} \\
\text { - } \text { Bidest. Water }\end{array}$ & $\begin{array}{l}28.5 \mathrm{~g} \\
800 \mathrm{ml}\end{array}$ \\
\hline Stock B (acid): & $\begin{array}{l}\text { - } \mathrm{NaH}_{2} \mathrm{PO}_{4} \cdot x \mathrm{H}_{2} \mathrm{O} \\
\text { - } \text { Bidest. water } \\
\text { use stock (B) to set stock (A) to } \mathrm{pH} 7.4\end{array}$ & $\begin{array}{l}8.3 \mathrm{~g} \\
300 \mathrm{ml}\end{array}$ \\
\hline PFA-solution & $\begin{array}{l}\text { - } \text { PFA } \\
\text { - } \text { Bidest. water } \\
\rightarrow \text { dissolve at } 60^{\circ} \mathrm{C} \\
\text { - } 10 \mathrm{M} \mathrm{NaOH} \\
\text { Fill up to } 500 \mathrm{ml} \text { with bidest. water, } \\
\text { let cool down } \\
\text { - Add phosphate buffer }\end{array}$ & $\begin{array}{l}40 \mathrm{~g} \\
300 \mathrm{ml} \\
6-8 \text { drops to clear solution } \\
500 \mathrm{ml}\end{array}$ \\
\hline Rose bengal & $\begin{array}{l}\text { - } 0.9 \% \mathrm{NaCl} \\
\text { - } \quad \text { Rose bengal }\end{array}$ & $\begin{array}{l}10 \mathrm{ml} \\
100 \mathrm{mg}\end{array}$ \\
\hline $\begin{array}{l}\text { Sucrose solution } \\
10 \%: \\
30 \%:\end{array}$ & $\begin{array}{l}\text { - Sucrose } \\
\text { - Sucrose } \\
\text { Fill up to } 100 \mathrm{ml} \text { with } 0.1 \mathrm{M} \mathrm{PB}\end{array}$ & $\begin{array}{l}10 \mathrm{~g} \\
30 \mathrm{~g}\end{array}$ \\
\hline
\end{tabular}




\section{RESULTS}

\section{Abstract stroke study}

Most of the results of the following chapters 3.1 and 3.2 were already published (Greifzu et al., 2011, Global impairment and therapeutic restoration of visual plasticity mechanisms after a localized cortical stroke. Proc Natl Acad Sci USA 108(37): 15450-15455) and will be presented here in an extended and more detailed version. The following abstract is adopted from the published paper (Greifzu et al. 2011).

We tested the influence of a photothrombotic (PT) lesion in the somatosensory cortex on plasticity in the mouse visual system and the efficacy of anti-inflammatory treatment to rescue compromised learning. To induce plasticity mechanisms, we monocularly deprived (MD) 3 months old mice. In control animals, MD induced an increase of visual acuity of the open eye and an ocular dominance (OD) shift towards this eye. In contrast, after PT, there was neither an enhancement of visual acuity nor an OD-shift. However, OD-plasticity was still present in the hemisphere contralateral to the lesion. Anti-inflammatory treatment and a 2 week delay PT and MD could restore sensory learning but not OD-plasticity. We conclude that (i) both sensory learning after PT and cortical plasticity in the surround of a cortical lesion are impaired; (ii) most likely a transient inflammation is responsible for impaired sensory learning, suggesting anti-inflammatory treatment with ibuprofen as a useful adjuvant therapy to support rehabilitation following stroke; and (iii) OD-plasticity cannot be just a local process and nonlocal influences are probably more important than previously assumed.

\subsection{Impairment of visual plasticity after a cortical stroke}

\subsubsection{Localization and size of the photothrombotic lesion}

To model an ischemic infarct, we used the photothrombosis model (Watson et al. 1985) and induced a local cortical lesion in the left hemisphere (Figure 23). Seven days after induction of the PT-lesion, the brain was removed, cut into $40 \mu \mathrm{m}$ thick sections and Nissl-stained for analysis of lesion size and location. The lesion center was on average $1.3 \pm 0.06 \mathrm{~mm}$ anterior to the primary visual cortex (V1), $1.9 \pm 0.0 \mathrm{~mm}$ lateral to the midline (Figure $23 \mathrm{~A}, \mathrm{~B}$ ) and $0.9 \pm 0.06 \mathrm{~mm}$ posterior to the bregma. The destroyed area corresponded primarily to the hind 
limb region (Paxinos \& Franklin 2001). On average, PT-lesions had a diameter of $1.1 \pm 0.05 \mathrm{~mm}$ medio-laterally and $0.9 \pm 0.04$ anterio-posteriorly.

To test whether there was an influence of lesion size and location on the two different analyzed plasticity measurements in PT-mice after MD, both, values of visual acuity enhancement and the ocular dominance index (ODI) after MD were correlated with (i) lesion volume, (ii) lesion depth (affected layers), and (iii) the distance of the lesion center from V1. The lesion volume was calculated as a cylinder with the lateral-medial, anterior-posterior diameter and the depth of the lesion and was on average $0.73 \pm 0.20 \mathrm{~mm}^{3}$. Notably, lesion size and location did neither correlate with the enhancement of visual acuity nor with the ODI (Figure 24). Pearson correlation coefficients were as follows: Visual acuity enhancement after MD: volume: $r=-0.531$; affected layers: $r=-0.229$; distance from $V 1: r=-0.070(P>0.05$ for all). ODI: volume: $\mathrm{r}=-0.016$; affected layers: 0.129; distance from V1: -0.606 (P > 0.05 for all). Moreover, the size of the lesion was neither influenced by treatment with the antiinflammatory drug ibuprofen nor by time $(1,2$, or 3 weeks after lesion induction, respectively; all $\mathrm{P}>0.05$, t-test). The brain tissue of sham treated mice as well as the contralateral hemispheres of the PT-animals displayed no macroscopic or microscopic structural changes in the Nissl-stained sections. 

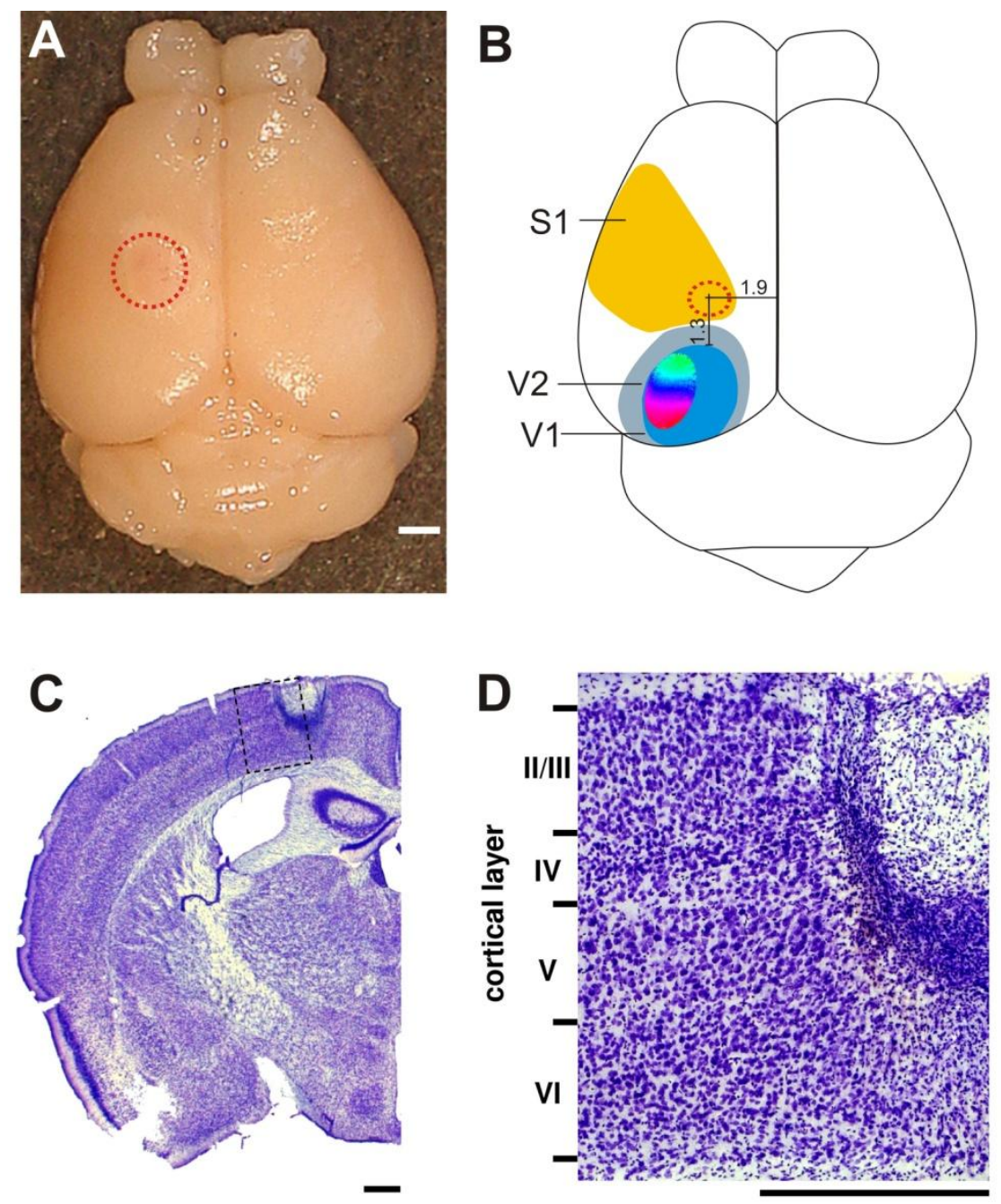

Figure 23: Location and size of the photothrombotic lesion in the mouse cortex. (A) Top view of a mouse brain with a photothrombotic lesion (red dotted circle) in the left hemisphere. Scale bar: $1 \mathrm{~mm}$. (B) Scheme from the same view showing the average lesion size and location. The lesion center was located in the left primary somatosensory cortex (S1), on average $1.3 \mathrm{~mm}$ anterior to the anterior border of $\mathrm{V} 1$ and $1.9 \mathrm{~mm}$ lateral to the midline. A retinotopic map from the binocular part of $\mathrm{V} 1$ acquired with optical imaging of intrinsic signals was interposed to visualize the spatial relation of the lesion and V1. (C) Left hemisphere of a Nissl-stained frontal section of a mouse brain displaying the lesion center 7 days after lesion induction. The lesion is clearly visible and restricted to the cortex, not extending into underlying white matter or subcortical regions. Scale bar: $500 \mu \mathrm{m}$. (D) A higher magnification image of the lesion border (region marked by the black rectangle in C) shows the intact tissue and cortical layering in the perilesional zone. Scale bar: $500 \mu \mathrm{m}$. Modified from Greifzu et al. (2011). 


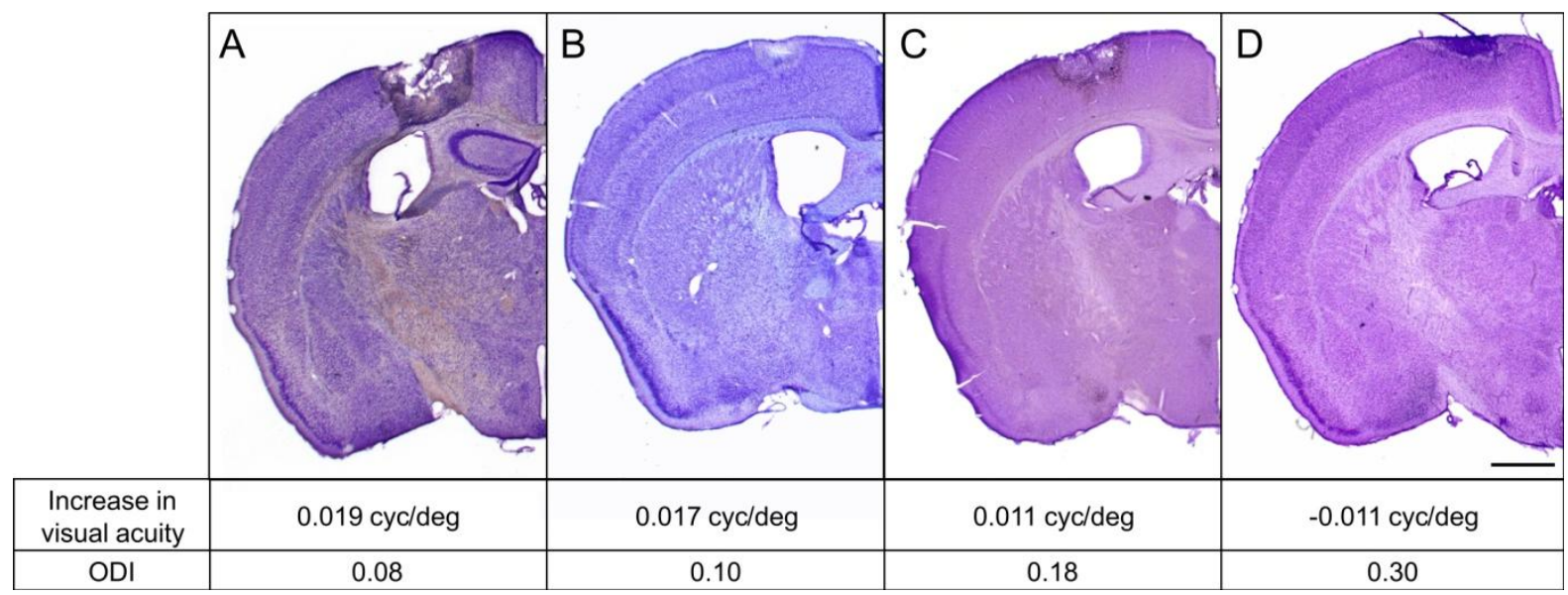

Figure 24: The photothrombotic lesion size did neither correlate with the enhancement of visual acuity of the open eye after MD nor with the ODI after MD. Shown are examples of Nissl-stained frontal brain sections of PT-mice with MD (day 7), and the corresponding increase in visual acuity (visual acuity at day 7 minus visual acuity before MD) and the ODI of the individual animal. In this experimental group (PT+MD) sensory learning (increase in visual acuity) and OD-plasticity was diminished. This diminished plasticity did not correlate with the lesion size. Interestingly, it was possible to observe an increase of visual acuity of the open eye and a low ODI after MD in a mouse with a big lesion (A). Mice with a smaller lesion might not show plasticity at all (D) or just slight plasticity (B, C). Data of the mouse shown in (A) was excluded from the analysis because the lesion extended into the white matter. Scale bar: $1 \mathrm{~mm}$.

\subsubsection{Photothrombosis abolished enhancement of vision after MD}

It is known that MD induces an increase of the optokinetic response (enhancement of visual acuity and contrast sensitivity, sensory learning) of the non-deprived eye in normal (nonlesioned) adult mice (Prusky et al. 2006; Lehmann \& Löwel 2008). Prusky et al. (2006) showed that visual acuity improvement depends on the activity the in visual cortex ipsilateral to the deprived eye, while activity in visual cortex contralateral to the deprived eye was necessary for the maintenance of the increased visual acuity after deprived eye re-opening. we separately tested the influence of a MD of the eye ipsi- or contralateral to the lesioned hemisphere using the optomotor system developed by Prusky et al. (2004). MD was induced directly after PT. In the following 7 days, both visual acuity and contrast sensitivity were measured behaviorally.

$M D$ of the right eye. In control animals, visual acuity was on average $0.38 \pm 0.003$ cycles per degree $(\mathrm{cyc} / \mathrm{deg})$ for the left and $0.38 \pm 0.003 \mathrm{cyc} / \mathrm{deg}$ for the right eye $(\mathrm{n}=9)$. Because there was no significant difference between values of the two eyes $(\mathrm{P}>0.05$, t-test $)$, we pooled values for further analyses. In PT-animals without MD, visual acuity was also not significantly different between left $(0.37 \pm 0.003 \mathrm{cyc} / \mathrm{deg})$ and right $(0.37 \pm 0.003 \mathrm{cyc} / \mathrm{deg})$ eyes $(\mathrm{P}<0.05$, t-test, $\mathrm{n}=13)$, and values were also pooled. Thus, a lesion in one hemisphere 
did not cause a significant difference between the visual acuity of the individual eyes. In control animals, baseline visual acuity (without MD) was $0.38 \pm 0.003 \mathrm{cyc} / \mathrm{deg}$ on day 1 and remained stable for 7 days $(P>0.05$, Bonferroni-adjusted t-test, $n=9$ ) (Figure 25, Figure 26 A). After MD, visual acuity of the open eye of control mice increased significantly from $0.39 \pm 0.003 \mathrm{cyc} / \mathrm{deg}$ on day 1 to $0.46 \pm 0.01 \mathrm{cyc} / \mathrm{deg}$ on day 7 ( $\mathrm{P}<0.001$, Bonferroniadjusted t-test, $\mathrm{n}=10$ ) (Figure 25, Figure $26 \mathrm{~B}$ ), this was significantly different from control animals without $\mathrm{MD}\left(\mathrm{F}_{1,17}=110.61, \mathrm{P}<0.001\right.$, ANOVA). In contrast, animals with a PTlesion showed no significant enhancement of visual acuity after MD (day 1: $0.38 \pm 0.01 \mathrm{cyc} / \mathrm{deg}$; day 7: $0.39 \pm 0.01 \mathrm{cyc} / \mathrm{deg} ; \mathrm{P}>0.05$, t-test, $\mathrm{n}=9$ ) (Figure 25, Figure $26 \mathrm{D})$ and thus visual acuities were not significantly different from PT-animals without MD (day 1: $0.37 \pm 0.003 \mathrm{cyc} / \mathrm{deg}$; day $7: 0.37 \pm 0.003 \mathrm{cyc} / \mathrm{deg} ; \mathrm{n}=13 ; \mathrm{F}_{1,20}=5.23, \mathrm{P}>0.05$, ANOVA) (Figure 25, Figure 26 C). This was surprising because the hemisphere ipsilateral to the deprived (right) eye, that is supposed to be responsible for the increase in visual acuity (Prusky et al. 2006), was not lesioned.

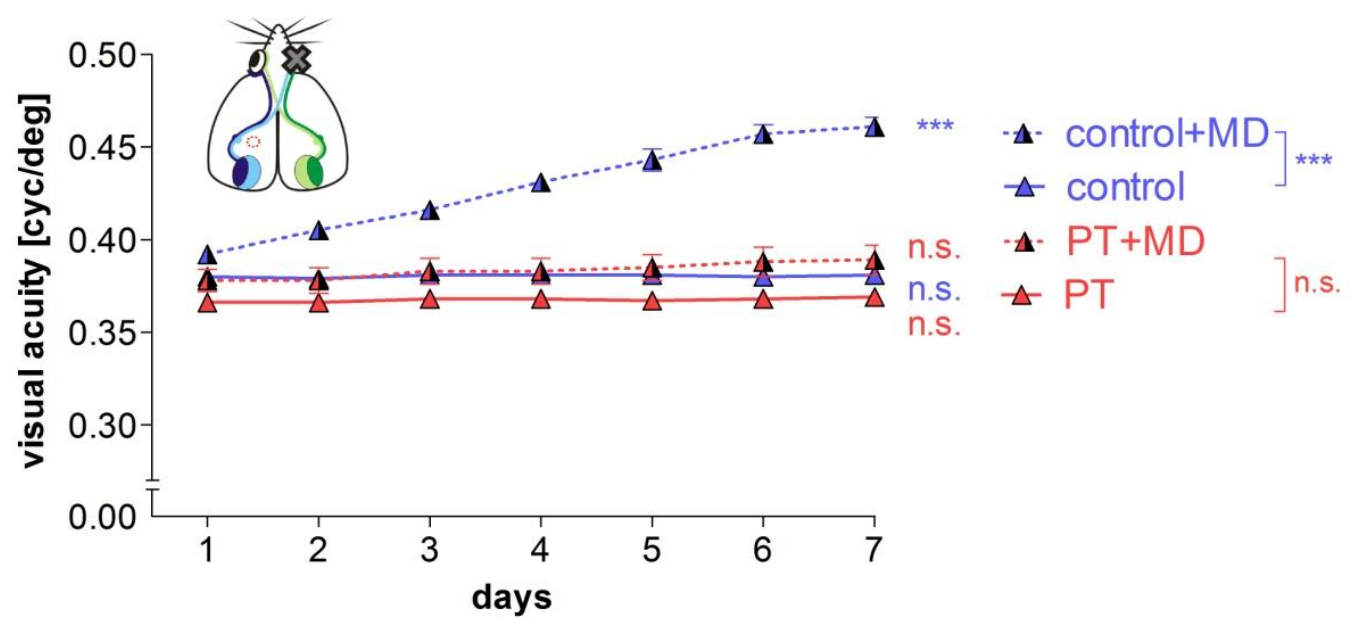

Figure 25: A PT-lesion outside the visual cortex, in the somatosensory cortex, abolished visual acuity improvement after MD in mice. Spatial frequency selectivity of the optokinetic response in cycles per degree (cyc/deg) plotted against days. In control animals, during 7 days of MD (control+MD), visual acuity of the open eye significantly increased. In contrast, in PT-animals, MD failed to induce improvement (PT+MD). Modified from Greifzu et al.(2011). 

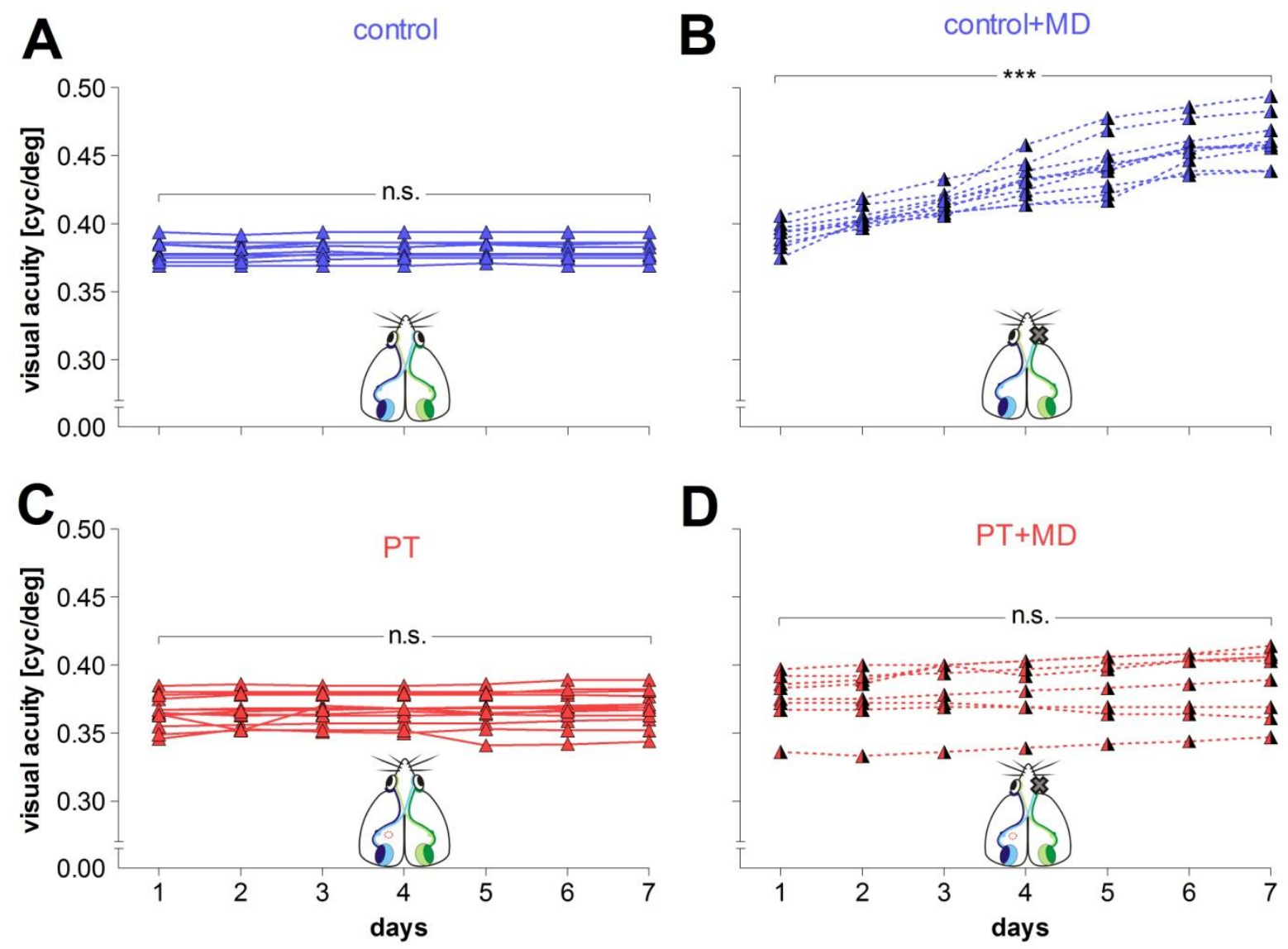

Figure 26: Visual acuity of individual control (A and B) and PT mice (C and D) without and with MD (+MD). Each line represents one animal. The visual acuity of control (A) and PT-mice (C) without MD remained stable over 7 days. After MD the visual acuity of the open eye of control mice increased significantly (B). However, in mice with a PT-lesion there was no significant increase in the visual acuity after MD (D).

Additionally, we analyzed the gain on the baseline, i.e. the increase of visual acuity values in \% from the baseline value on day 1 (before MD) until the end of MD (day 7). This comparison confirmed that the increase of visual acuity of the open eye after MD was significantly higher in control mice $(17.7 \pm 1.1 \% \triangleq 0.07 \pm 0.004 \mathrm{cyc} / \mathrm{deg})$ than in PT-mice $(3.0 \pm 0.9 \% \triangleq 0.01 \pm 0.004 \mathrm{cyc} / \mathrm{deg})\left(\mathrm{F}_{1,17}=109.30, \mathrm{P}<0.001\right.$, ANOVA) (Figure 27) 


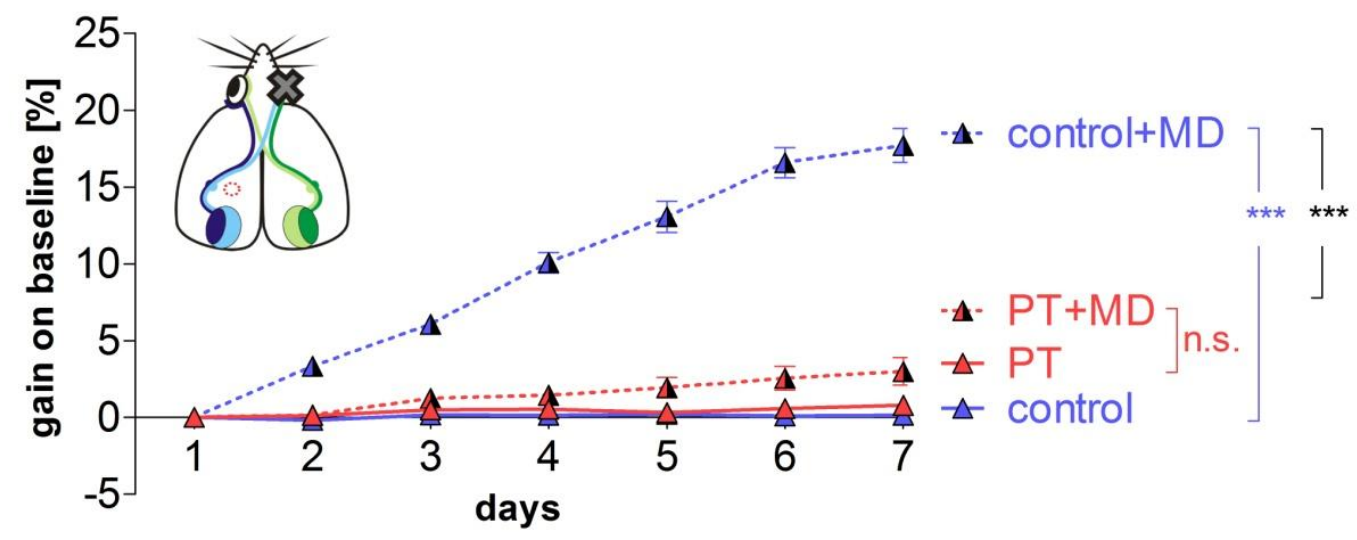

Figure 27: Gain on baseline of visual acuity. Shown is the increase of visual acuity values in $\%$ from the baseline value on day 1 (before MD) until the end of MD (day 7). The increase in visual acuity of the open eye after MD was significantly higher in control- than in PT-mice.

Likewise, a PT-lesion abolished the enhancement of contrast sensitivity after MD. Contrast sensitivity was also analyzed with the virtual reality optomotor setup (Prusky et al. 2004) and measured at 6 different spatial frequencies. Contrast sensitivity curves of the two eyes of control and PT-animals were not significantly different and pooled for further analyses, respectively $(\mathrm{P}>0.05$, t-test). Without $\mathrm{MD}$, contrast sensitivities of control and PT-animals remained stable over time (control/PT: $\mathrm{P}>0.05$, Bonferroni-adjusted t-test, $\mathrm{n}=9 / 13$ ) (Figure 29 A, C). After MD in control animals, contrast sensitivity of the open eye was significantly enhanced ( $\mathrm{P}<0.001$, Bonferroni-adjusted t-test) (Figure 28, Figure $29 \mathrm{~B}$ ) at $0.064 \mathrm{cyc} / \mathrm{deg}$

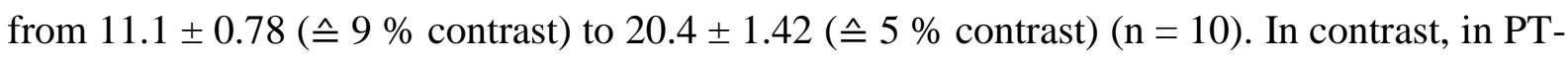
animals, MD had no enhancing effect on contrast sensitivity: values on day 1 (11.4 \pm 1.44$)$ were not significantly different from values on day 7 (11.2 \pm 2.05 ; P > 0.05, Bonferroniadjusted t-test, $n=9$ ) (Figure 28, Figure 29 D). Values for contrast sensitivity of measured spatial frequencies can be found in Table 4. 


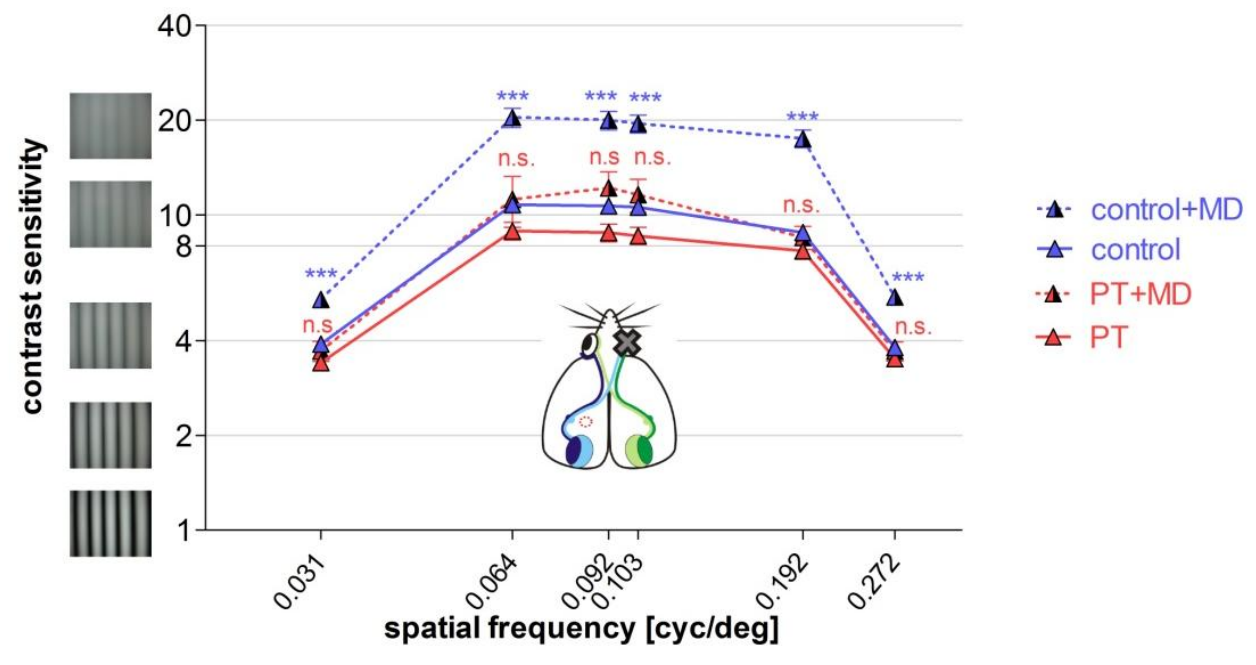

Figure 28: A PT-lesion outside the visual cortex prevented the use-dependent enhancement of contrast sensitivity of the open eye after MD. Contrast sensitivity was analyzed using a virtual reality optomotor setup (Prusky et al. 2004). Contrast sensitivity was measured at 6 different spatial frequencies. Illustrated values are from day 7 (after MD in deprived animals). In control animals, contrast sensitivity was $11.1 \pm 0.8(\mathrm{n}=10)$ and significantly increased to $20.4 \pm 1.4$ at a spatial frequency of $0.064 \mathrm{cyc} / \mathrm{deg}$ (control+MD). In contrast, in PT-animals contrast sensitivity did not increase significantly after MD (PT+MD). Modified from Greifzu et al. (2011).

A

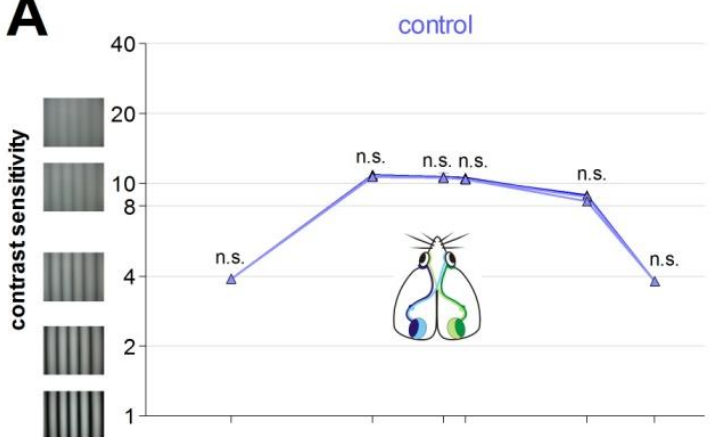

C

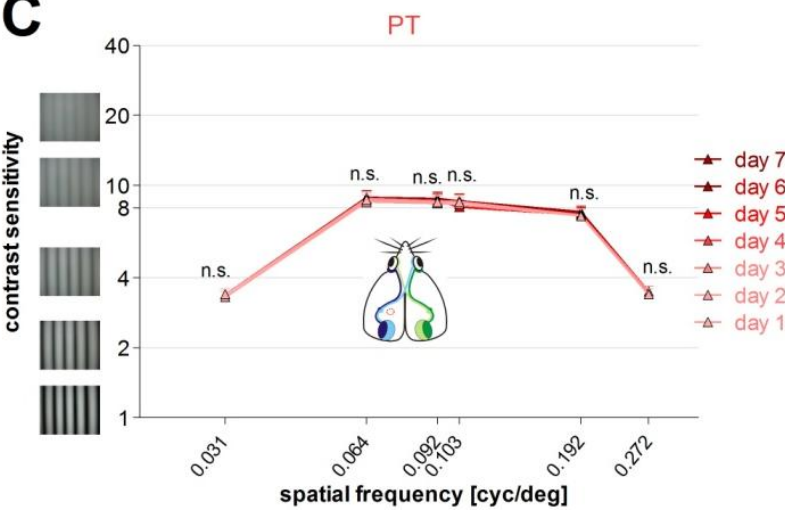

B

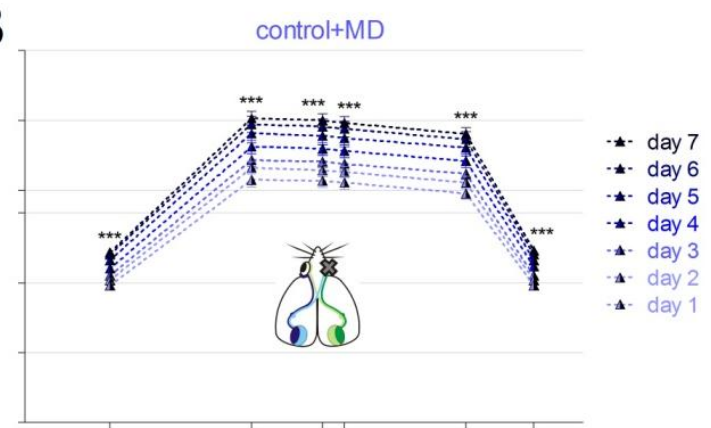

D

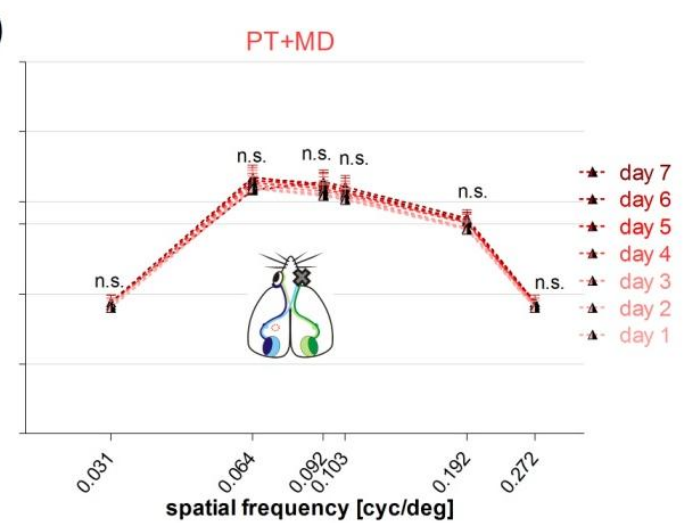

Figure 29: Mean contrast sensitivities for the individual days and spatial frequencies. (A) In control animals without MD contrast sensitivity remained stable over 7 days. (B) After MD the contrast sensitivity of the open eye increased significantly at all measured spatial frequencies. (C) In PT-lesioned mice without MD contrast sensitivity remained also stable. (D) In contrast to control mice, in PT-mice there was no significant increase in the contrast sensitivity of the open eye after MD. 
Table 4: Contrast sensitivity of the open eye increased significantly in control, but not in PTmice after MD. Average contrast values \pm S.E.M. on day 1 and day 7 after MD and statistical significance for the increase over these days (Bonferroni-adjusted t-test).

\begin{tabular}{|c|c|c|c|}
\hline Frequencies [cyc/deg] & Contrast sensitivity day 1 & Contrast sensitivity day 7 & $\mathrm{P}$ \\
\hline control+MD & & & \\
\hline 0.031 & $3.9 \pm 0.07$ & $5.4 \pm 0.14$ & $<0.001$ \\
0.064 & $11.1 \pm 0.78$ & $20.4 \pm 1.42$ & $<0.001$ \\
0.092 & $11.0 \pm 0.74$ & $20.0 \pm 1.36$ & $<0.001$ \\
0.103 & $10.8 \pm 0.70$ & $19.5 \pm 1.29$ & $<0.001$ \\
0.192 & $9.7 \pm 0.55$ & $17.7 \pm 1.14$ & $<0.001$ \\
0.272 & $3.9 \pm 0.06$ & $5.5 \pm 0.15$ & $<0.001$ \\
\hline $\mathrm{PT}+\mathrm{MD}$ & & & \\
\hline 0.031 & $3.5 \pm 0.23$ & $3.7 \pm 0.27$ & $>0.05$ \\
0.064 & $11.4 \pm 1.44$ & $11.2 \pm 2.05$ & $>0.05$ \\
0.092 & $10.6 \pm 1.22$ & $12.2 \pm 1.54$ & $>0.05$ \\
0.103 & $10.2 \pm 1.12$ & $11.6 \pm 1.39$ & $>0.05$ \\
0.192 & $7.6 \pm 0.58$ & $8.5 \pm 0.72$ & $>0.05$ \\
0.272 & $3.5 \pm 0.22$ & $3.7 \pm 0.26$ & $>0.05$ \\
\hline
\end{tabular}

MD of the left eye. In PT-animals, the was also no significant visual acuity improvement of the open eye after left eye MD $(n=10)$ compared to control animals with $\mathrm{MD}\left(\mathrm{F}_{1,18}=60.71\right.$, $\mathrm{P}<0.001$, ANOVA $)$ and was indistinguishable from PT-animals with right eye $\mathrm{MD}\left(\mathrm{F}_{1,17}=\right.$ 3.61, P > 0.05, ANOVA). Similarly, enhancement of contrast sensitivity was also significantly diminished after left eye MD $(\mathrm{n}=10)$ compared to control animals with MD $\left(\mathrm{F}_{1,18}=56.96, \mathrm{P}<0.001\right.$, ANOVA $)$ and was indistinguishable from PT-animals with right eye $\mathrm{MD}\left(\mathrm{F}_{1,17}=8.61, \mathrm{P}>0.05\right.$, ANOVA $)$.

Concluding, irrespective of the side of MD, visual acuity and contrast sensitivity enhancement were always abolished, suggesting a non-local, possibly brain-wide disturbance caused by the PT-lesion.

\subsubsection{OD-plasticity was impaired in the surround of a PT-lesion}

Using optical imaging of intrinsic signals we analyzed the OD-plasticity in the binocular region of V1 of the lesioned hemisphere.

In control animals without MD, visual stimulation of the contralateral eye induced stronger cortical activation than ipsilateral eye stimulation (Figure $30 \mathrm{~A}$ ). In the optically imaged greyscale coded response magnitude maps of the binocular region of V1, activity patches evoked by stimulation of the contralateral were darker than those after stimulation of the ipsilateral eye (Figure $30 \mathrm{~A}$ ). The histogram of ODIs was centered right of zero, the two-dimensional 
OD-map, in which the ODI-values are color-coded for each pixel, displayed warm red colors and the ODI was positive (average ODI: $0.28 \pm 0.03, \mathrm{n}=7$, Figure $31 \mathrm{~A}$ ), indicating contralateral dominance. After 7 days of MD in control animals the response magnitude maps of both contra- (deprived) and ipsilateral (open) eye were equally dark (Figure $30 \mathrm{~B}$ ). The histogram of the OD-score was shifted to the left, colder blue colors prevailed in the OD-map and the average ODI was with $0.03 \pm 0.02(\mathrm{n}=9)$ significantly lower than in control mice without MD ( $\mathrm{P}<0.001$, Bonferroni-adjusted t-test) (Figure $31 \mathrm{~A})$. Thus, the MD induced a significant OD-shift towards the open eye in control mice. PT-mice without MD showed also contralateral dominance: activity patches evoked by stimulation of the contralateral eye were darker than those after ipsilateral eye stimulation (Figure $30 \mathrm{C}$ ). The histogram of ODIs was centered right of zero, the two-dimensional OD-map displayed warm red colors and the ODI was positive (average ODI: $0.19 \pm 0.01, n=13$, Figure $31 \mathrm{~A}$ ). In contrast to control mice, in PT-animals, MD did not induce a significant OD-shift: the binocular visual cortex remained dominated by input from the contralateral eye (Figure $30 \mathrm{D}$ ). After MD, activity patches induced by stimulation of the contralateral (deprived) eye were still darker than those of the ipsilateral (open) eye. The histogram of ODIs was still centered right of zero, the twodimensional OD-map displayed warm red colors and the ODI was positive (average ODI: $0.17 \pm 0.02, \mathrm{n}=10$, Figure $31 \mathrm{~A})$. The ODI was not significantly different from PT-animals without MD (P>0.05, Bonferroni-adjusted t-test) (Figure $31 \mathrm{~A})$, but different from control animals after MD ( $\mathrm{P}<0.05$, Bonferroni-adjusted t-test).

Concluding, MD induced a significant OD-shift towards the open eye in control-, but not PTmice. 


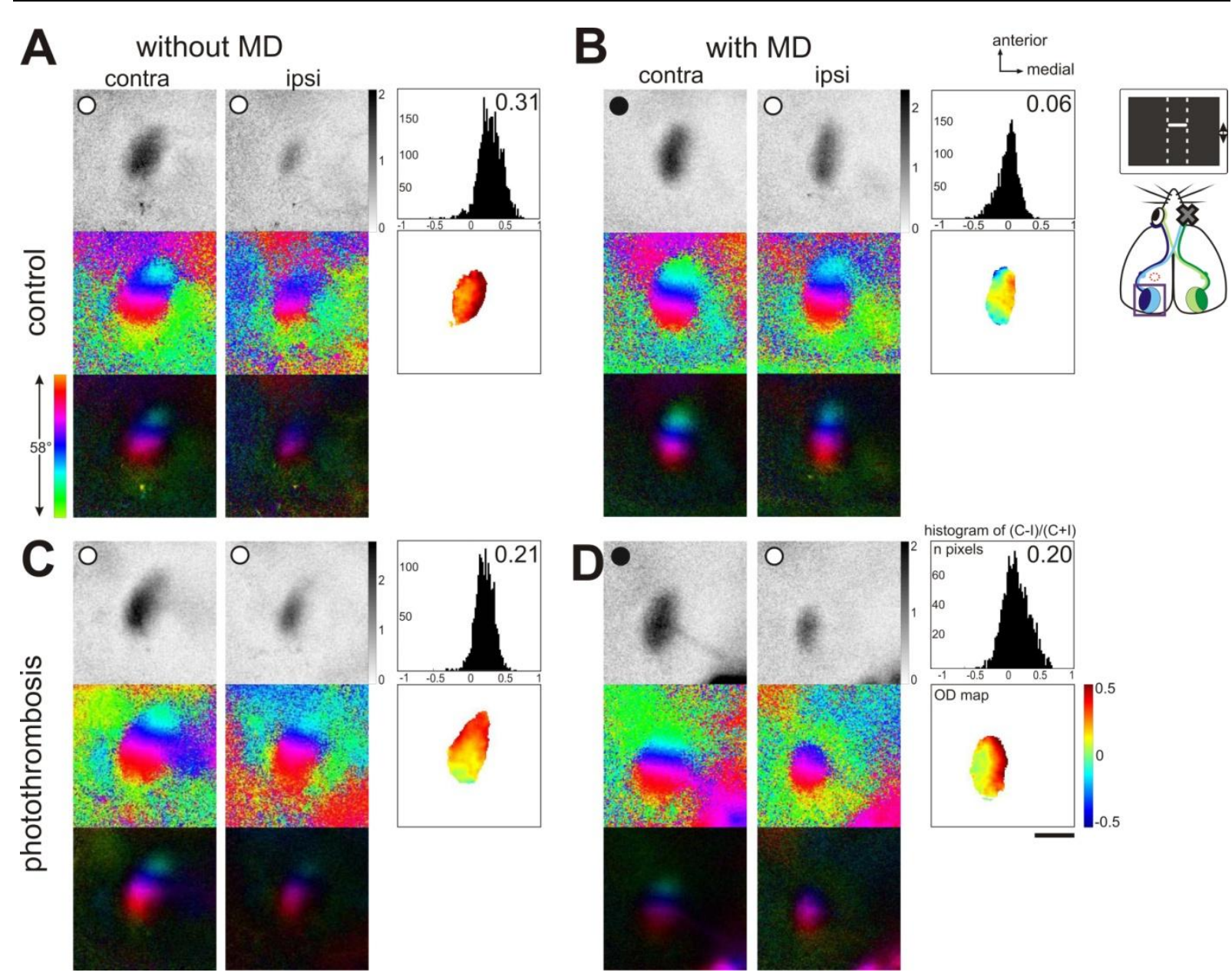

Figure 30: A PT-lesion outside the visual cortex prevented OD plasticity. Optically imaged maps of responses after contra- and ipsilateral (ipsi) eye stimulation in the binocular region of mouse visual cortex in both control animals (A, B) and in mice with PT (C, D). Maps without MD are illustrated in the left column (A and C), maps after MD in the right column (B, D). Both gray-scale coded response magnitude maps and their quantification (top), and color-coded phase and polar maps of retinotopy (middle, bottom) are illustrated. The magnitude of the optical responses is illustrated as fractional change in reflection $\times 10^{4}$. For each experiment, the histogram of OD-scores, the average OD-index (ODI), and the corresponding two-dimensional OD-maps are included (ODI values are color-coded: blue represents negative, red positive values). Note that without MD, activity patches evoked by stimulation of the contralateral eye were consistently darker than those after stimulation of the ipsilateral eye, two-dimensional OD-maps display warm colors, and the average ODI is positive, indicating contralateral dominance (A and C). In contrast, MD for 7 days in control (B) but not PTanimals (D) induced a significant OD-shift toward the open eye: the response magnitude maps of both contra- (deprived) and ipsilateral (open) eye were equally dark, the histogram of OD-scores shifted to the left, the ODI was 0.06 compared to 0.31 without MD, and colder colors prevailed in the OD-map. In PT-animals with MD (D), the response magnitude maps of the deprived (contralateral) eye were still darker than those of the open (ipsilateral) eye; the histogram of OD-scores and the average ODI were unchanged and warm colors still prevailed in the OD-map. Scale bar: $1 \mathrm{~mm}$. Modified from Greifzu et al. (2011). 

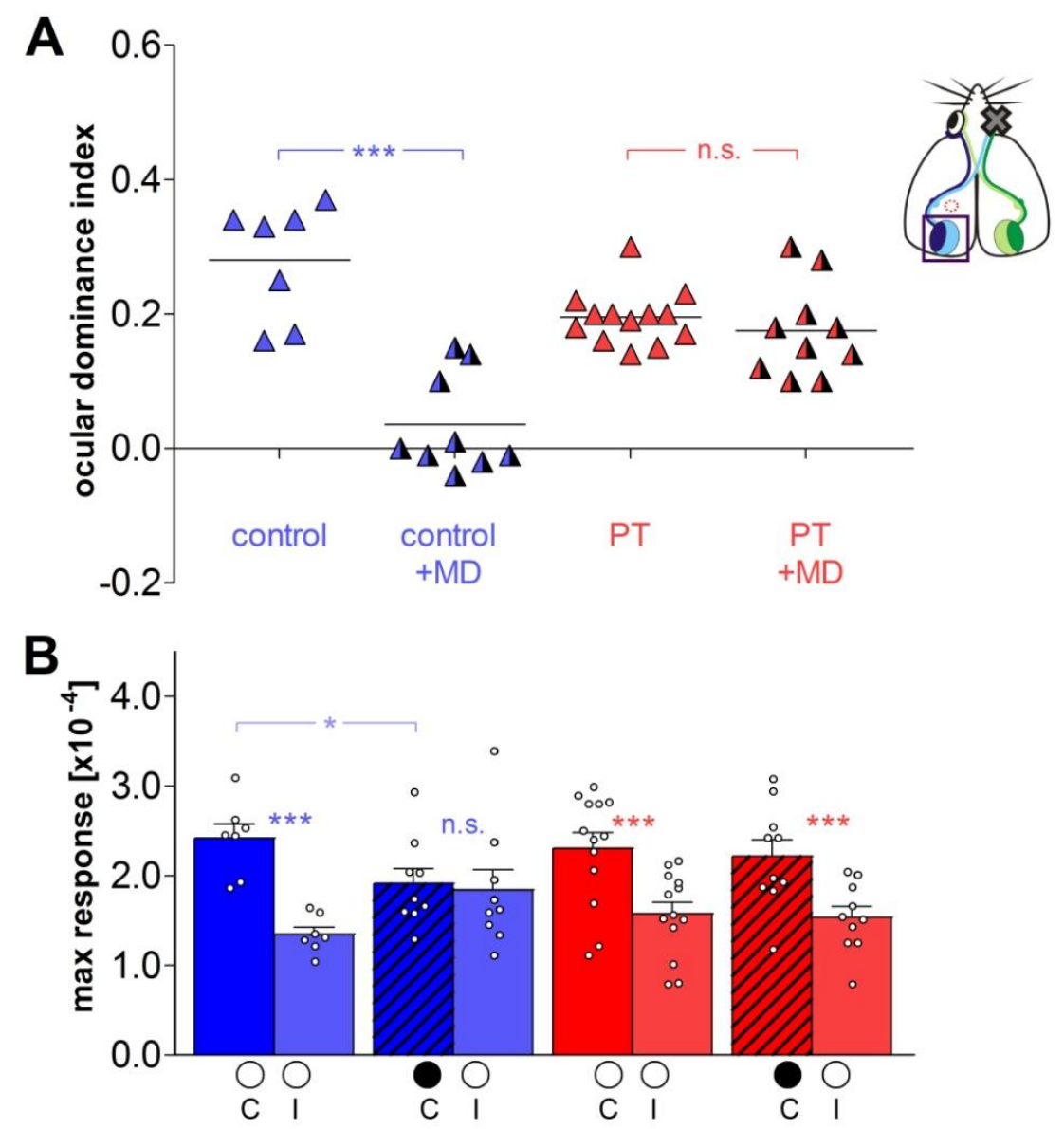

Figure 31: OD-plasticity was absent in the lesioned hemisphere. (A) OD-indices of the left (lesioned) hemisphere after MD of the contralateral eye (see inset). Symbols represent ODI values of individual animals; means are marked by horizontal lines. In control animals, MD induced a significant reduction of ODIs (control+MD). In contrast, in PT-animals, ODIs were not changed after MD (compare PT with PT+MD). (B) Maximum response magnitude in V1 expressed as fractional change in light reflectance elicited by stimulation of the contralateral (C) or ipsilateral (I) eye. Unfilled circles symbolize open eyes, black filled circles closed eyes. In control animals, cortical activation after visual stimulation of the contralateral eye was significantly higher than after ipsilateral eye stimulation, reflecting the dominance of the contralateral eye. After MD of the contralateral eye, response strengths of the two eyes were no longer significantly different: deprived-eye responses were slightly reduced and open-eye responses were slightly increased compared. In contrast, after MD in PT-animals, the response strengths of both eyes remained significantly different. Modified from Greifzu et al. (2011).

\section{Changes in response strength after $M D$}

To better understand the mechanisms underlying the difference in OD-plasticity between control and PT-animals, we compared the maximum response magnitude in V1 after stimulation of the contra- or ipsilateral eye, respectively (Figure $31 \mathrm{~B}$ ). In control animals, cortical activation in V1 was significantly higher after visual stimulation of the contralateral $(2.42 \pm 0.16)$ compared to the ipsilateral eye $(1.34 \pm 0.08 ; \mathrm{P}<0.001, \mathrm{t}$-test, $\mathrm{n}=7)$. After $\mathrm{MD}$ of the contralateral eye, response strengths of the two eyes were no longer significantly 
different $(\mathrm{P}>0.05$, t-test): Open-eye responses were slightly but non-significantly enhanced $(1.84 \pm 0.23 ; \mathrm{P}>0.05$, t-test, $\mathrm{n}=9)$ and the response strength after deprived-eye stimulation was slightly decreased $(1.92 \pm 0.16 ; \mathrm{P}<0.05$, t-test $)$. In contrast, in PT-mice, cortical activation after deprived-eye stimulation remained significantly higher than after open-eye stimulation (deprived/open: $2.22 \pm 0.18 / 1.53 \pm 0.12, \mathrm{n}=10 ; \mathrm{P}<0.001$, $\mathrm{t}$-test), as in PT-mice without MD (contra/ipsi: $2.31 \pm 0.17 / 1.57 \pm 0.13, \mathrm{n}=13$; $\mathrm{P}<0.001$, t-test).

Since it was a bit surprising that in sham treated control animals the OD-shift was mainly mediated by a decrease in response strength after deprived eye stimulation, instead of an increase of the open eye responses as reported in control C57BL/6 mice before (Sato \& Stryker 2008), we performed additional chronic imaging experiments. We imaged control animals before MD, performed MD after the imaging experiment and imaged again 7 days later. The results in two chronic experiments were similar as in the acute experiments: In one animal, cortical responses to deprived eye stimulation were reduced without a change in open eye responses (activity after stimulation of the deprived eye: before MD 2.55, after MD 1.73; open eye: before MD 1.76, after MD 1.84) and in another animal, it was the other way around (activity after stimulation of the deprived eye: before MD 1.43, after MD 1.35; open eye: before MD 1.09, after MD 1.49) (Figure 32).

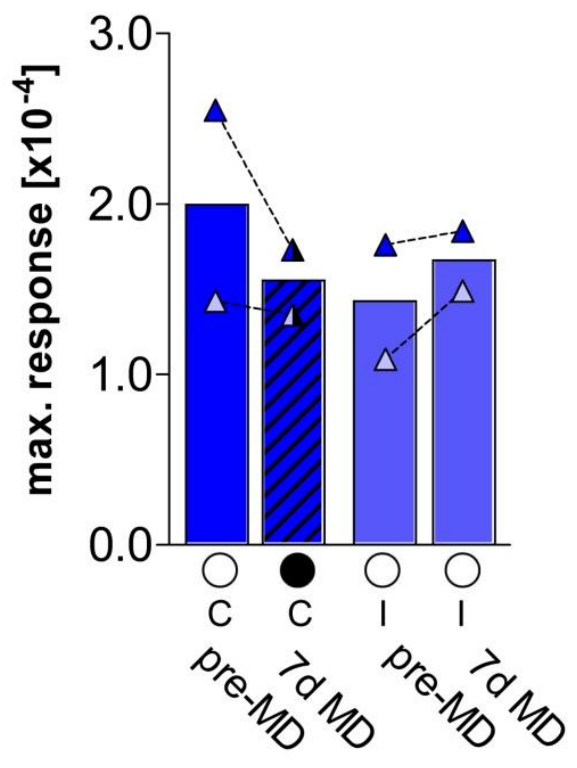

Figure 32: Maximum response strength in chronic optical imaging experiments in control mice before and after MD. Control animals were imaged before MD and 7 days after MD to determine whether the OD-shift after MD in control mice is mediated by a decrease in closed eye responses or increase in open eye responses. Shown are the results of two chronic experiments. On the left are depicted the maximum responses after contralateral eye stimulation, on the right after ipsilateral eye stimulation. In one animal, cortical responses to deprived eye stimulation were reduced without a change in open eye responses and in the other animal; it was the other way around. 


\subsubsection{OD-plasticity was impaired in the surround of a PT-lesion - electrophysiological} results

To verify the results of the optical imaging experiments with a method that does not rely on changes in blood flow, we performed electrophysiological recordings. The recordings were performed by Dr. Klaus Kreikemeier at the University of Jena. The data confirmed the results obtained by optical imaging of intrinsic signals: there was no OD-plasticity in the surround of a PT-lesion. Extracellular recordings from control- and PT-mice with MD were obtained from the binocular region of V1 (as determined by intrinsic signal optical imaging). We recorded from 88 neurons in the binocular region of V1 in the left hemisphere of 4 control and 5 lesioned animals after 7 days of MD. Cells were assigned to OD classes according to the 7class scheme of Hubel and Wiesel (1962). In normal, non-lesioned adult wild-type mice the visual cortex is dominated by input from the contralateral eye (Dräger 1975; Gordon \& Stryker 1996) (Figure 32 B). After 7 days of MD, our control animals showed a more balanced OD distribution (Figure 32 C), similar to that described in the literature (Figure 32 A). In contrast, the visual cortex of PT-animals was still dominated by the contralateral (deprived) eye (Figure $32 \mathrm{D}$ ). As an indicator of the relative responses of the cortex to visual stimulation of the contra- and ipsilateral eye, we additionally calculated the contralateral bias index (CBI), which ranges from 0 (complete ipsilateral dominance) to 1 (complete contralateral dominance) (Gordon \& Stryker 1996). In control mice after MD, the average CBI was 0.47 (39 cells, 4 mice) (Figure 32 C), reflecting a more balanced OD-distribution and thus an OD-shift. In contrast, in PT-animals, the average CBI was 0.68 (49 cells, 5 mice) (Figure $32 \mathrm{D}$ ), still reflecting contralateral dominance. CBI-distributions were significantly different between PT and control animals after MD $(\mathrm{P}<0.0001 ; \chi 2$ test $)$.

Concluding, the electrophysiological measurements verified the optical imaging results that there was no OD-plasticity in the lesioned hemisphere of PT-mice. 

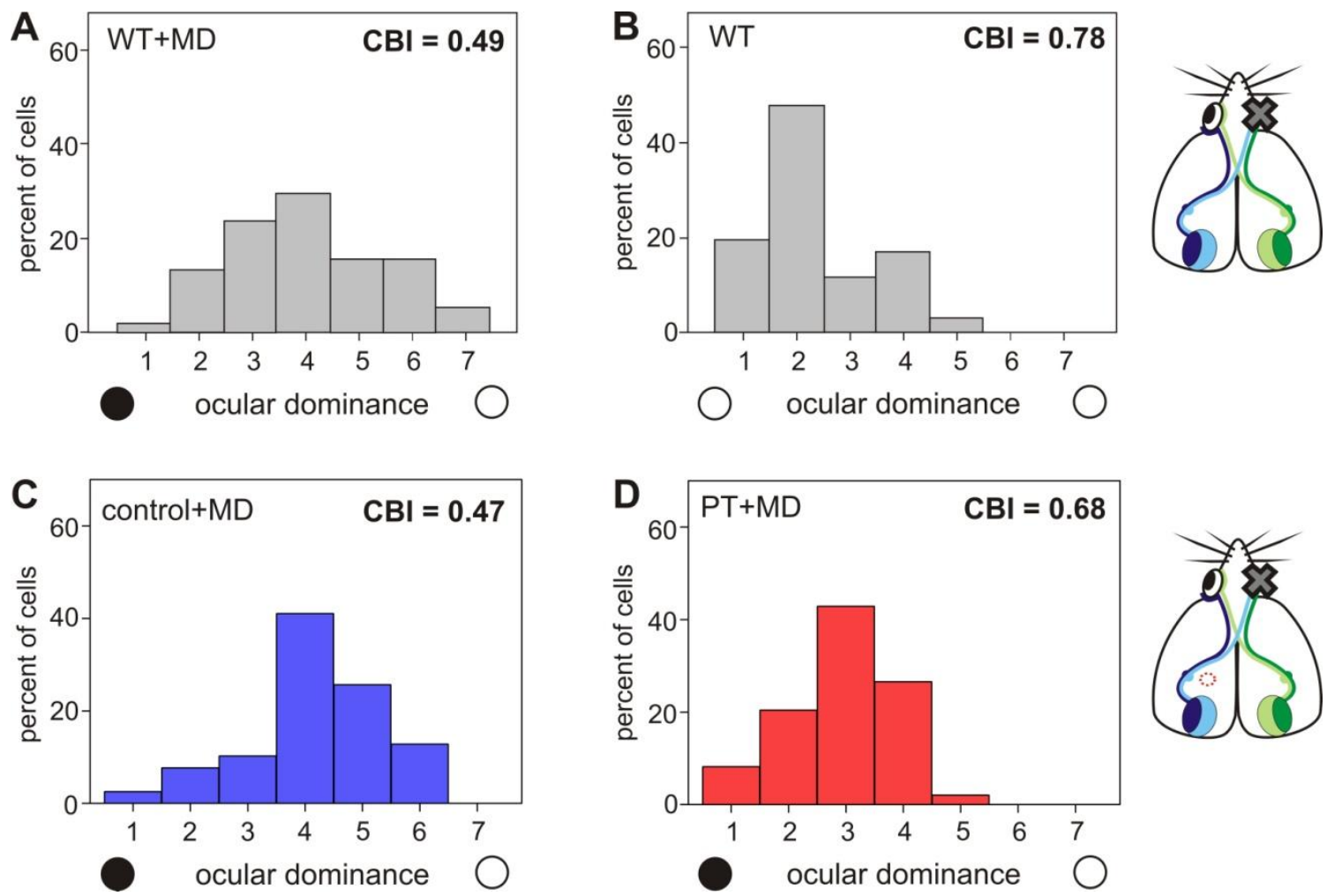

Figure 33: No OD-plasticity in PT-animals: electrophysiological results. OD scores plotted against the percentage of cells and contralateral bias index (CBI, top right corner) of wild-type mice (WT) in the literature (A, B; modified from Hensch (1998)) and in our experiments (C, D; modified from Greifzu et al. (2011)). (B) In normal, non-lesioned adult mice the visual cortex is dominated by input from the contralateral eye. After 7 days of MD of the contralateral eye (solid black circle), our control animals showed a more balanced OD-distribution (C), similar to that described in the literature (A). In contrast, the visual cortex of PT-animals was still dominated by the contralateral (deprived) eye (D). As an indicator of the relative responses of the cortex to visual stimulation of the contra- and ipsilateral eye, the CBI was calculated, which ranges from 0 (complete ipsilateral dominance) to 1 (complete contralateral dominance). (C) In control mice, the average CBI was 0.47 , reflecting a more balanced OD-distribution and thus an OD-shift. (B) In contrast, in PT-animals, the average CBI was 0.68 , still reflecting contralateral dominance.

\subsubsection{OD-plasticity after PT was still present in the non-lesioned hemisphere - distinct} effects of MD in the non-lesioned and lesioned hemisphere

In the data presented above (3.1.3) we showed that OD-plasticity after MD of the contralateral eye was absent in the lesioned hemisphere (Figure 35 D, E; Figure 36 A). To check whether the reduction of plasticity in the lesioned hemisphere was caused by global mechanisms affecting the entire brain, we next analyzed OD-plasticity in the non-lesioned hemisphere.

In the non-lesioned hemisphere OD-plasticity is still present in PT-mice, after MD of the contralateral, as well as after MD of the ipsilateral eye. 
Contralateral eye $M D$. In the optical imaging maps in the non-lesioned hemisphere of control (Figure $34 \mathrm{~A}$ ) and PT-mice (Figure $34 \mathrm{D}$ ) without MD activity patches evoked by stimulation of the contralateral eye were consistently darker than those after stimulation of the ipsilateral eye, two-dimensional OD-maps display warm colors, and the average ODI is positive, indicating contralateral dominance. MD of the contralateral eye for 7 days induced a significant OD-shift toward the open eye in control (Figure $34 \mathrm{~B}$ ) as well as in PT-mice (Figure $34 \mathrm{E}$ ): the response magnitude maps both contra- (deprived) and ipsilateral (open) eye were almost equally dark, the histogram of OD-scores shifted to the left and colder colors prevailed in the OD-map. In control mice the ODI significantly decreased from $0.22 \pm 0.05$ $(\mathrm{n}=6)$ to $0.05 \pm 0.01(\mathrm{n}=3)$ after $\mathrm{MD}(\mathrm{P}<0.05$, Bonferroni-adjusted t-test) (Figure $36 \mathrm{~B})$. In PT-mice the ODI significantly decreased, from $0.20 \pm 0.02(\mathrm{n}=10)$ to $0.11 \pm 0.02(\mathrm{n}=10)$ ( $\mathrm{P}<0.05$, Bonferroni-adjusted t-test) (Figure $36 \mathrm{~B}$ ). Noteworthy, after MD the ODI in PTmice was still significantly higher than in control mice (pooled group of control mice with contralateral MD, ODI $=0.04 \pm 0.02, \mathrm{n}=12 ; \mathrm{P}<0.001$, Bonferroni-adjusted t-test). Thus, the OD-shift in the non-lesioned hemisphere after contralateral MD was less strong in PT-mice than in control mice.

Ipsilateral eye $M D$. After MD of the ipsilateral eye there was also a significant OD-shift towards the open eye, i.e. an even stronger contralateral dominance, in PT-mice (Figure 34 F): the response magnitude maps after contra- and ipsilateral eye stimulation were even more different, the histogram of OD-scores shifted to the right and even more warm colors prevailed in the OD-map. The ODI significantly increased significantly to $0.36 \pm 0.03$ ( $\mathrm{n}=7$; $\mathrm{P}<0.01$, Bonferroni-adjusted t-test) (Figure $36 \mathrm{~B}$ ). In control mice the OD-shift after ipsilateral MD was not significantly increased $(0.36 \pm 0.03, \mathrm{n}=10 ; \mathrm{P}>0.05$, Bonferroniadjusted t-test) (Figure $36 \mathrm{~B}$ ), but a tendency of an increased ODI and an OD-shift towards the open eye was also visible (Figure $34 \mathrm{C}$ ). Hence, OD-plasticity was even slightly enhanced in PT-mice.

Concluding, OD-plasticity was still present in the non-lesioned hemisphere, indicating that the reduction of OD-plasticity in the lesioned hemisphere must be a rather specific process and cannot be caused by a general decline of plasticity mechanisms in the entire brain.

Since we observed slightly enhanced OD-plasticity after ipsilateral eye MD in the nonlesioned hemisphere, we also analyzed OD-plasticity after ipsilateral eye MD in the lesioned hemisphere. Surprisingly, in contrast to contralateral eye MD (Figure 35 D, E; Figure 36 A); ipsilateral eye $M D$ could induce a significant OD-shift in the lesioned hemisphere (Figure 
$36 \mathrm{C}$ ). Compared to PT-mice without MD (Figure $35 \mathrm{D}$ ), after ipsilateral MD the response magnitude maps after contra- and ipsilateral eye stimulation were even more different, the histogram of OD-scores was shifted to the right and yet more warm colors prevailed in the OD-map. In contrast to control mice, where no significant OD-shift could be observed (without MD: $0.28 \pm 0.03, \mathrm{n}=7$; with $\mathrm{MD}_{\text {ipsi: }} 0.34 \pm 0.07, \mathrm{n}=4$; $\mathrm{P}>0.05$, Bonferroniadjusted t-test) (Figure $35 \mathrm{~A}, \mathrm{C}$; Figure $36 \mathrm{C}$ ), the ODI in PT-mice significantly increased from $0.19 \pm 0.01(\mathrm{n}=13)$ to $0.33 \pm 0.03(\mathrm{n}=10)(\mathrm{P}<0.001$, Bonferroni-adjusted t-test $)$.

Summarizing, MD of the "weak", ipsilateral eye in PT-animals could still induce a significant OD-shift even in the lesioned hemisphere. 


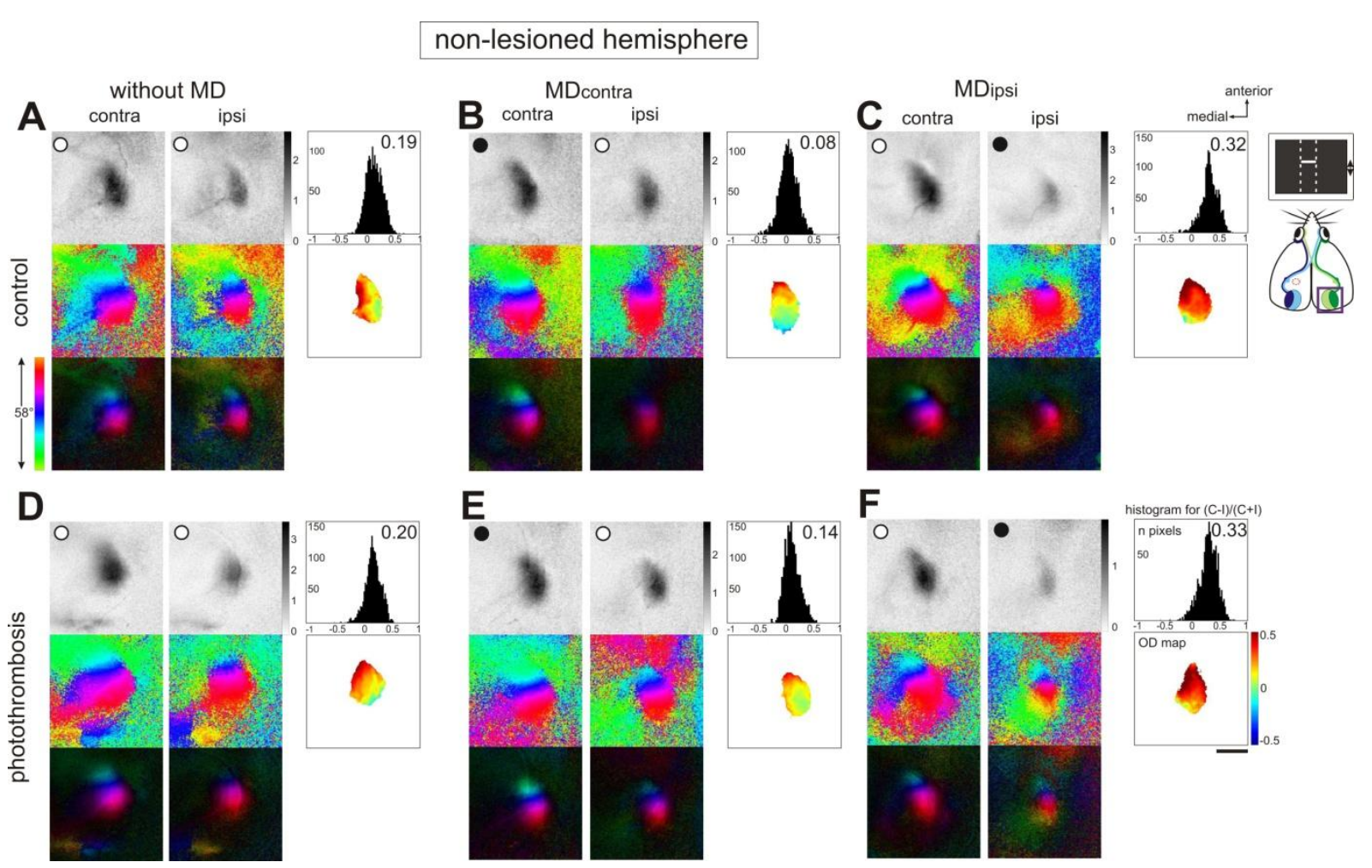

Figure 34: OD-plasticity after PT is still present in the non-lesioned hemisphere, after contralateral and ipsilateral MD. Optically imaged maps of responses after contra- and ipsilateral (ipsi) eye stimulation in the binocular region of mouse visual cortex in the non-lesioned right hemisphere. Shown are control animals (A, B, C) and mice with PT (D, E, F). Maps without MD are illustrated in the left column (A, D), maps after MD of the contralateral eye in the middle column (B, E) and maps after ipsilateral eye MD in the right column $(\mathrm{C}, \mathrm{F})$. Both gray-scale coded response magnitude maps and their quantification (top), and color-coded phase and polar maps of retinotopy (middle, bottom) are illustrated. The magnitude of the optical responses is illustrated as fractional change in reflection $\times 10^{4}$. For each experiment, the histogram of OD-scores, the average OD-index (ODI), and the corresponding two-dimensional OD-maps are included (ODI values are color-coded: blue represents negative, red positive values). Black filled circles on the response magnitude maps indicate the deprived eye, unfilled circles non-deprived eyes. Note that without MD, activity patches evoked by stimulation of the contralateral eye were consistently darker than those after stimulation of the ipsilateral eye, two-dimensional OD-maps display warm colors, and the average ODI is positive, indicating contralateral dominance in control and PT-mice (A, D). MD of the contralateral eye for 7 days induced a significant OD-shift toward the open eye in control (B) as well as in PT-mice (E): the response magnitude maps both contra- (deprived) and ipsilateral (open) eye were almost equally dark, the histogram of OD-scores shifted to the left, the ODI was reduced and colder colors prevailed in the OD-map. After MD of the ipsilateral eye there was also a significant OD-shift towards the open eye in PT-mice (F): the response magnitude maps after contra- and ipsilateral eye stimulation were even more different, the histogram of OD-scores shifted to the right, the ODI was increased and even more warm colors prevailed in the OD-map. In control mice the OD-shift after ipsilateral MD was not significant, but a tendency of an increased ODI and an OD-shift towards the open eye was also visible (C). Scale bar: $1 \mathrm{~mm}$. 


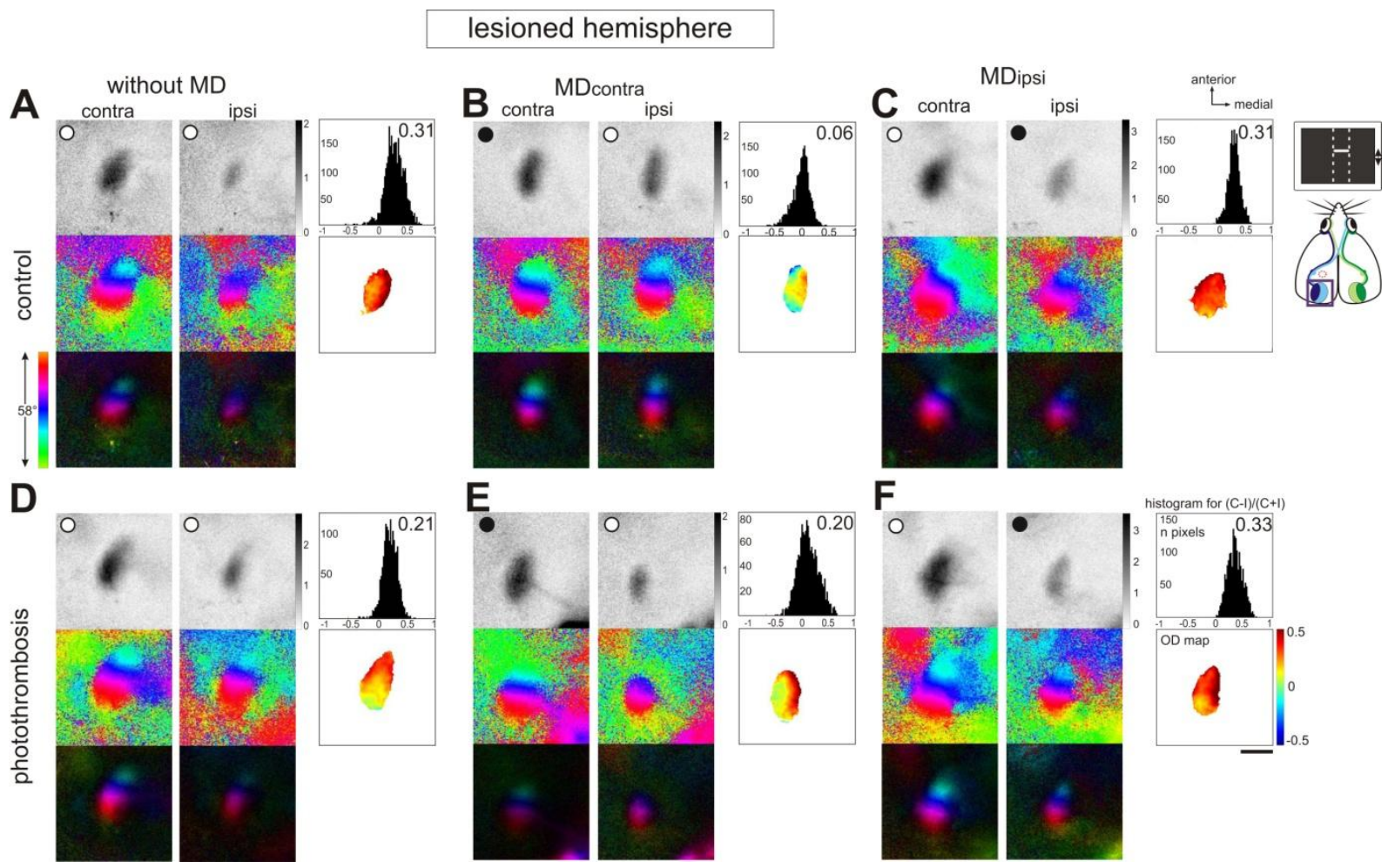

Figure 35: In the lesioned hemisphere OD-plasticity after PT is diminished after contralateral MD, but still present after ipsilateral MD. Optically imaged maps of responses after contra- and ipsilateral (ipsi) eye stimulation in the binocular region of mouse visual cortex in the lesioned left hemisphere. Shown are both, control animals (A, B, C) and mice with PT (D, E, F). Maps without MD are illustrated in the left column (A, D), maps after MD of the contralateral eye in the middle column $(\mathrm{B}, \mathrm{E})$ and maps after ipsilateral eye MD in the right column $(\mathrm{C}, \mathrm{F})$. Both gray-scale coded response magnitude maps and their quantification (top), and color-coded phase and polar maps of retinotopy (middle, bottom) are illustrated. The magnitude of the optical responses is illustrated as fractional change in reflection $\times 10^{4}$. For each experiment, the histogram of OD-scores, the average OD-index (ODI), and the corresponding two-dimensional OD-maps are included (ODI values are color-coded: blue represents negative, red positive values). Black filled circles on the response magnitude maps indicate the deprived eye, unfilled circles non-deprived eyes. Without MD, activity patches evoked by stimulation of the contralateral eye were consistently darker than those after stimulation of the ipsilateral eye, two-dimensional OD-maps display warm colors, and the average ODI is positive, indicating contralateral dominance in control and PT-mice (A, D). Contralateral eye MD for 7 days induced in control (B) but not PT-animals (E) a significant OD-shift toward the open eye. In control mice (B) the response magnitude maps of both contra- (deprived) and ipsilateral (open) eye were equally dark; the histogram of OD-scores shifted to the left, the ODI was reduced. In PT-animals with $\mathrm{MD}(\mathrm{E})$, the response magnitude maps of the deprived eye were still darker than those of the open eye; the histogram of OD-scores and the average ODI were essentially unchanged and warm colors still prevailed in the OD-map. However, after ipsilateral eye MD there was still a significant OD-shift towards the open eye observable in PT-mice (F): the response magnitude maps after contra- and ipsilateral eye stimulation were even more different, the histogram of OD-scores shifted to the right, the ODI was increased and even more warm colors prevailed in the OD-map. In control mice the ODshift after ipsilateral MD was not significant, but a tendency of an increased ODI and an OD-shift towards the open eye was also visible (C). Scale bar: $1 \mathrm{~mm}$. 


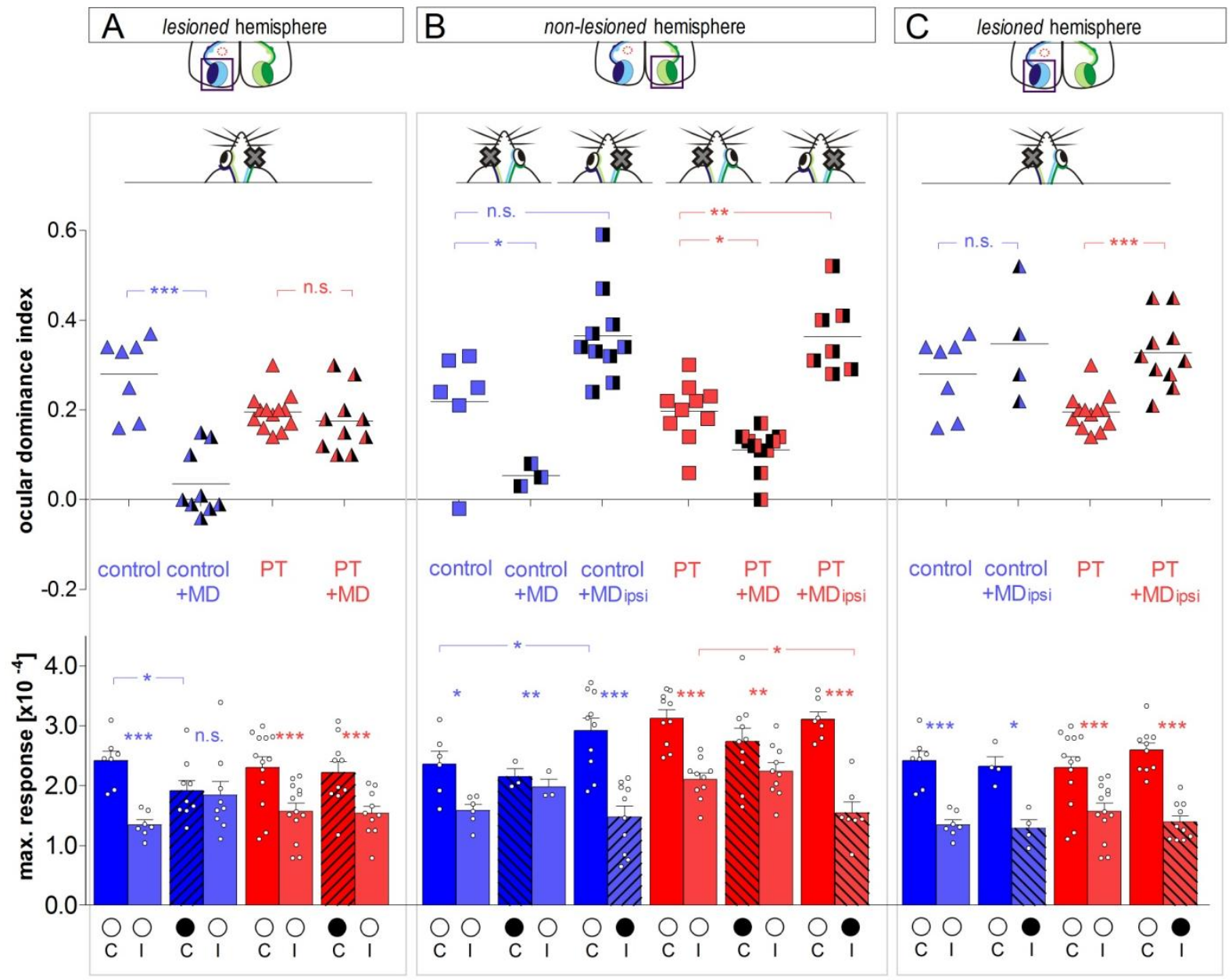

Figure 36: Distinct effects of MD in lesioned and non-lesioned hemisphere: OD-plasticity after PT still present in the non-lesioned hemisphere. Upper panel: OD-indices of the left (lesioned) and right (non-lesioned) hemisphere after deprivation of either the contralateral (MD) or the ipsilateral eye (MDipsi). Symbols represent ODI values of individuals; means are marked by horizontal lines. In the lower panel response magnitudes after stimulation of the contra (C) or ipsilateral (I) eye are shown for the respective groups. Filled circles represent deprived eyes, unfilled circles non-deprived eyes. (A) Lesioned hemisphere. In control animals, MD of the dominant contralateral eye lead to a significant OD-shift towards the open eye and cortical activation changed from being stronger after contralateral eye stimulation to a more balanced activation. In contrast, in PT-animals, stronger contralateral eye activation remained and no significant OD-shift could be observed. (B) Non-lesioned hemisphere. In control animals there was a significant OD-shift after contralateral MD, but not after ipsilateral MD. In contrast, in PT-animals we observed a significant OD-shift towards the open eye both after depriving the contra- and ipsilateral eye. (C) Lesioned hemisphere. Interestingly, MD of the "weak", ipsilateral eye in PT-animals could induce a significant OD-shift even in the lesioned hemisphere. Concluding, a PT-lesion selectively disturbs OD-plasticity in V1 of the lesioned hemisphere while OD-plasticity was preserved in the non-lesioned hemisphere.

\subsubsection{Simultaneous imaging in both hemispheres}

What differences might exist between the two hemispheres that could account for the differences in OD-plasticity mentioned in the paragraph above (3.1.5)? To approach this problem, we simultaneously recorded visual cortical activity of the two hemispheres, using a 
horizontal bar to stimulate both eyes equally strong at the same time. The strength of activation in the two hemispheres was compared by calculating a hemisphere activation quotient as the average of visual cortex activation of the right hemisphere divided by the average activation of the left hemisphere $\left(\right.$ activation $_{\text {right }}$ activation $_{\text {left }}$ ) after binocular stimulation. A quotient of 1 indicates that both hemispheres were activated evenly strong; a quotient less than 1 represents stronger activation of the left, which means in PT-mice the lesioned hemisphere; a quotient higher than 1 indicates stronger activation of the right (nonlesioned) hemisphere. In control animals, as expected, cortical activation in the visual cortex after binocular visual stimulation was the same in the left and right hemisphere: the evoked activity patches in the grey-scale coded response magnitude maps acquired by optical imaging were almost equally dark in both hemispheres (Figure $37 \mathrm{~A}$ ) and the hemisphere activation quotient was $0.90 \pm 0.06(n=8)$ (Figure 38). The same was true for control mice after MD: activity patches in V1 were similarly dark and the hemisphere activation quotient was $1.00 \pm 0.05(\mathrm{n}=10)$ (Figure $37 \mathrm{~B})$ and not significantly different from control mice without MD (P > 0.05, t-test) (Figure 38). In PT-animals with MD, V1 was also equally activated in both hemispheres (Figure $37 \mathrm{D})$ and the quotient showed the same $(1.06 \pm 0.04, \mathrm{n}=10)$. In contrast, in PT-animals without MD, visual stimulation induced a higher cortical activation in the non-lesioned compared to the lesioned hemisphere in PT-animals (Figure $37 \mathrm{C}$ ) and the quotient was $1.15 \pm 0.08(\mathrm{n}=14)$. This hemisphere activation quotient was significantly higher compared to control mice ( $\mathrm{P}<0.05$, t-test) (Figure 38). Thus, on average PT-mice showed a higher activation of the non-lesioned hemisphere than of the lesioned hemisphere. In all other experimental groups visual cortex activity in both hemispheres was almost equally strong (Figure $37 \mathrm{E}-\mathrm{M}$ ) and the hemisphere activation quotients did not differ from the respective comparison group (for all comparisons: $\mathrm{P}>0.05$, t-test) (Figure 38).

If the difference in the hemisphere activation quotients accounts for the difference in ODplasticity, there should be a correlation between the hemisphere activation quotients and ODIs. However, in PT+MD mice there was no correlation $(\mathrm{r}=-0.47, \mathrm{P}>0.05$, Pearson correlation).

Concluding, V1 of PT-mice showed a higher activation in the non-lesioned than the lesioned hemisphere, compared to control mice. But since the hemisphere activation quotient does not correlate with the ODI in PT-mice after MD, the difference in activity of the two hemispheres cannot account completely for the difference in OD-plasticity of control- and PT-mice. 


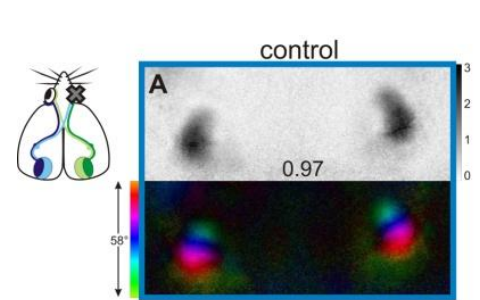

PT
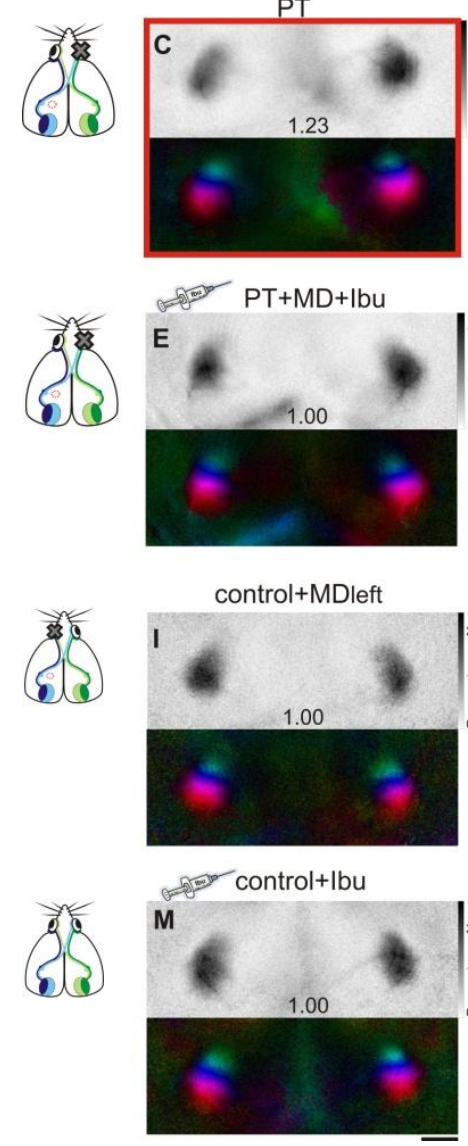

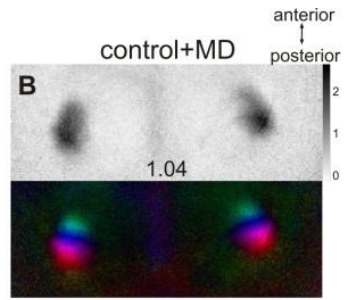

$\mathrm{PT}+\mathrm{MD}$
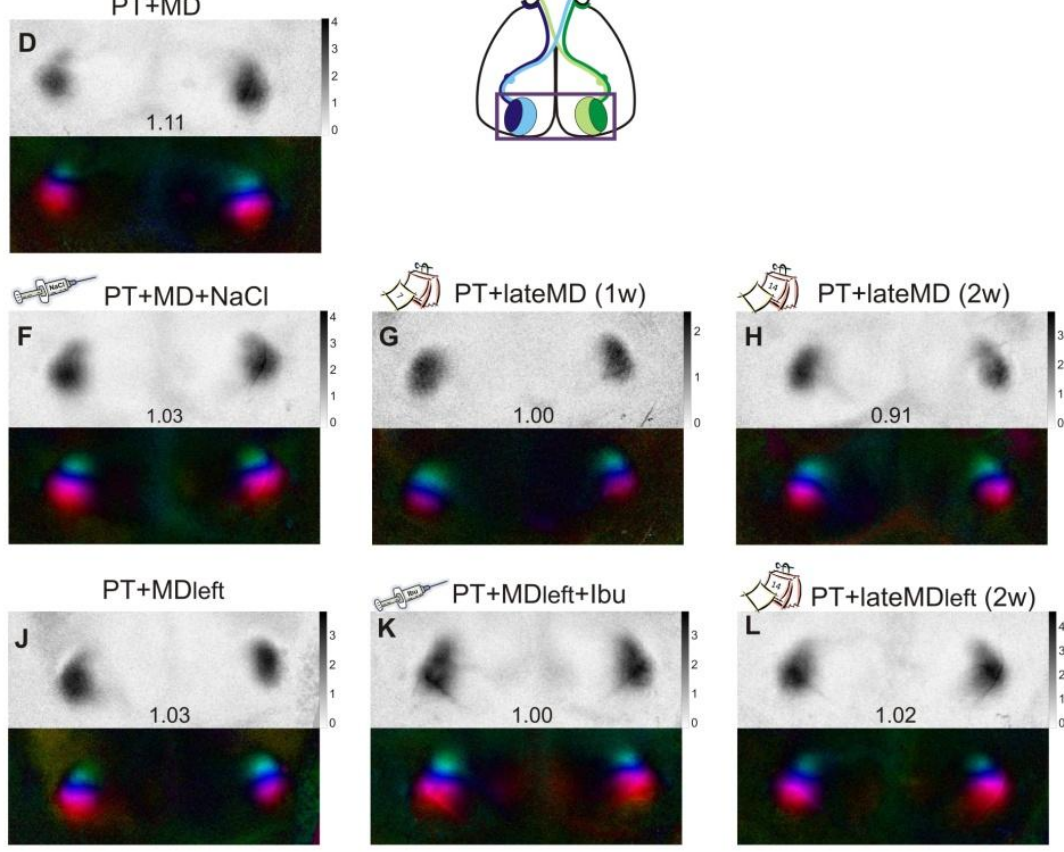

Figure 37: After a PT-lesion, the visual cortex was on average stronger activated in the nonlesioned compared to the lesioned hemisphere. Simultaneous optical imaging of cortical activity in the left and right hemispheres after visual stimulation of both eyes with a moving horizontal bar (see inset top right) in the different animal groups. Gray-scale coded response magnitude maps (upper) and color-coded polar maps of retinotopy (lower) are shown. The magnitude of the optical responses is illustrated as fractional change in reflection $\times 10^{4}$. The hemisphere activation quotient is displayed for each illustrated experiment. Note that in the illustrated PT-animal (C), the activity patch of the right (non-lesioned) hemisphere was darker than that of the left (lesioned) hemisphere and the activation quotient was 1.23, larger than in the illustrated control animal (0.97) (A). The activation of left and right hemisphere in the other experimental groups was similar and did not differ significantly from each other (see Figure 38). Scale bar: $1 \mathrm{~mm}$. 


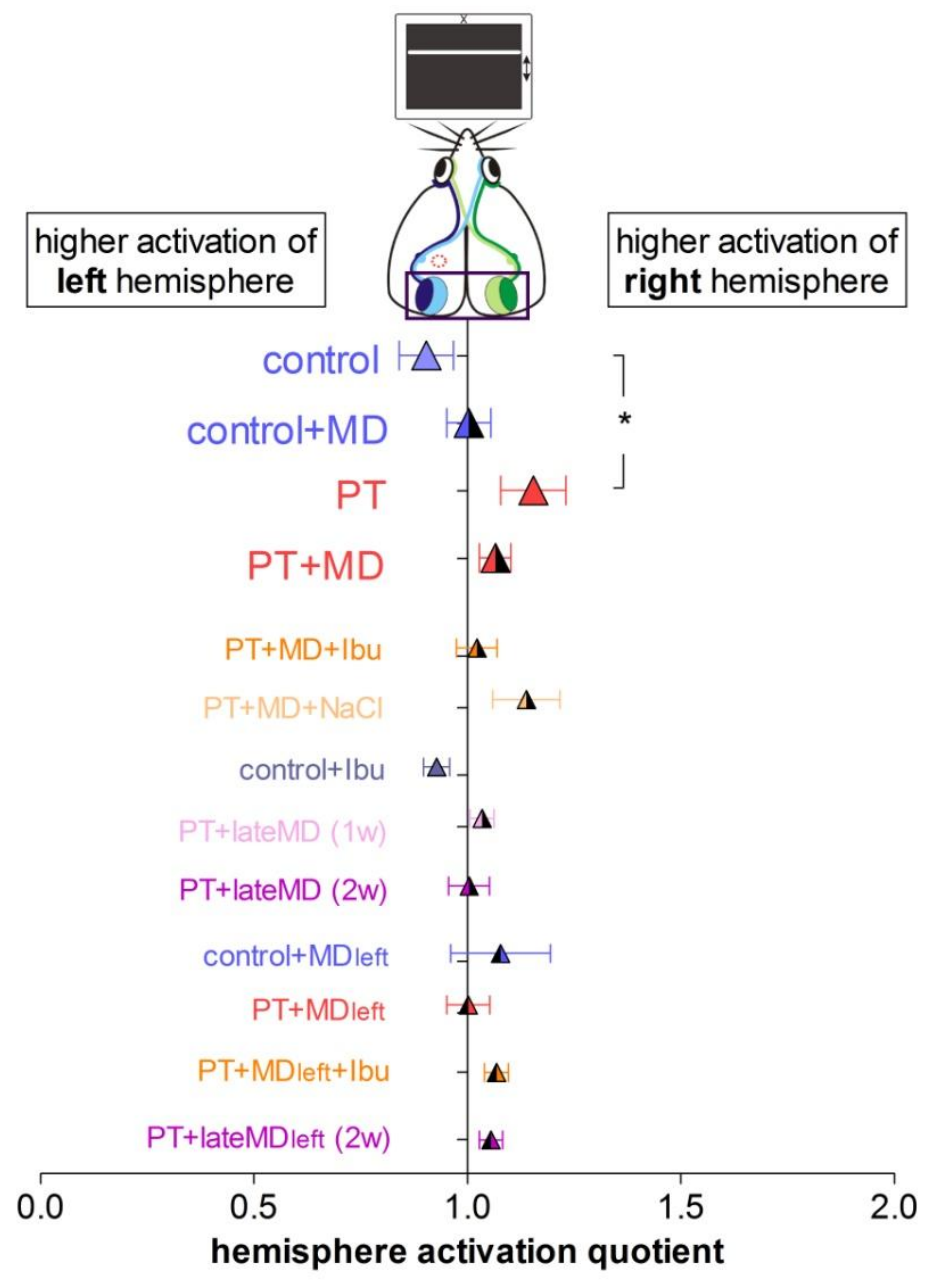

Figure 38: After a PT-lesion, visual cortex was on average stronger activated in the non-lesioned compared to the lesioned hemisphere The average hemisphere activation quotient of PT-animals was significantly higher than in controls, indicating a higher activation of the non-lesioned (right) hemisphere compared to the lesioned (left) hemisphere upon visual stimulation. The activation quotient of the other experimental groups did not differ significantly from each other. Modified from Greifzu et al. (2011).

\subsection{Therapeutic restoration of visual plasticity after a cortical stroke}

\subsubsection{Ibuprofen and late MD restored enhancement of vision after MD}

Ibuprofen restored enhancement of vision after MD in PT-mice

Since stroke has been shown to be accompanied by inflammation processes in the brain (Garcia 1975; Liu et al. 1994; Sairanen et al. 2001; Schroeter et al. 2002), we next tested whether anti-inflammatory treatment with the cyclooxygenase (COX)-inhibitor ibuprofen might rescue diminished plasticity after a PT-lesion. 
$M D$ of the right eye. In lesioned mice treated with ibuprofen $(\mathrm{n}=12)$ during MD, visual acuity of the open eye indeed increased significantly (day 1: $0.40 \pm 0.003 \mathrm{cyc} / \mathrm{deg}$; day 7: $0.46 \pm 0.01 \mathrm{cyc} / \mathrm{deg} ; \mathrm{P}<0.001$, Bonferroni-adjusted t-test) (Figure 39, Figure $40 \mathrm{~A}$ ). This increase was indistinguishable from non-lesioned animals with $\mathrm{MD}\left(\mathrm{F}_{1,20}=0.50, \mathrm{P}>0.05\right.$, ANOVA). In contrast, vehicle-injected $(\mathrm{NaCl})$ PT-animals $(n=6)$ did not improve significantly after MD (day 1: $0.39 \pm 0.005 \mathrm{cyc} / \mathrm{deg}$; day 7: $0.40 \pm 0.01 \mathrm{cyc} / \mathrm{deg} ; \mathrm{P}>0.05$, Bonferroni-adjusted t-test) (Figure $40 \mathrm{~B}$ ) and were indistinguishable from untreated PT-mice with $\mathrm{MD}\left(\mathrm{F}_{1,13}=1.51, \mathrm{P}>0.05\right.$, ANOVA) (Figure 39). Ibuprofen treatment itself had no effect on the visual acuity of control mice (day 1: $0.38 \pm 0.002 \mathrm{cyc} / \mathrm{deg}$; day 7: $0.38 \pm 0.002$ cyc/deg; $\mathrm{P}>0.05$, Bonferroni-adjusted t-test, $\mathrm{n}=6$ ) (Figure 39, Figure $40 \mathrm{C}$ ), which was indistinguishable from untreated control animals $\left(\mathrm{F}_{1,13}=0.39, \mathrm{P}>0.05\right.$, ANOVA $)$.

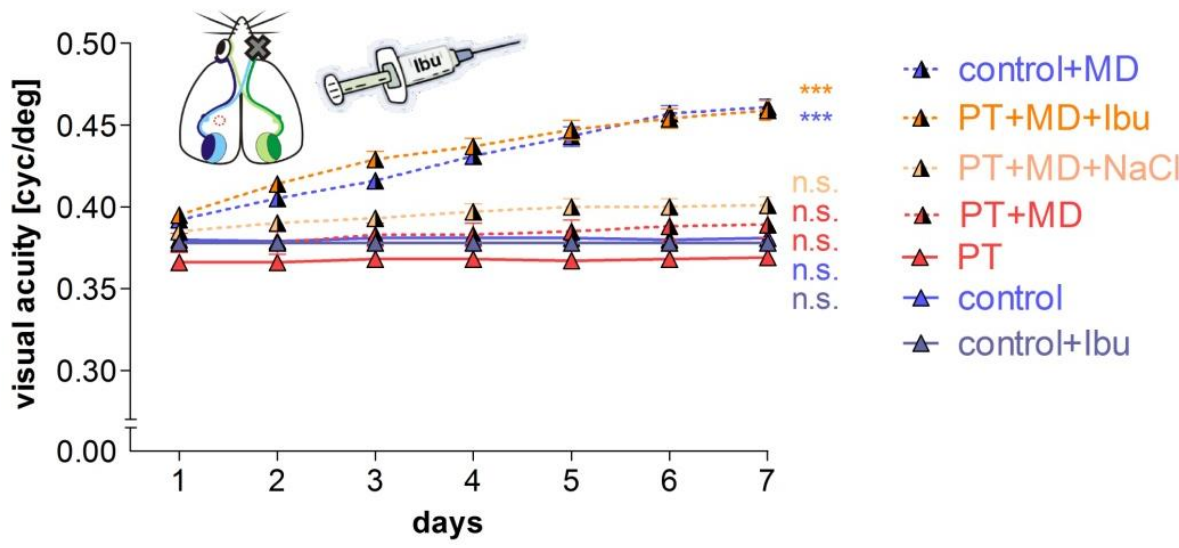

Figure 39: Ibuprofen rescued visual acuity improvement after MD in PT-mice. Spatial frequency selectivity of the optokinetic response in cycles per degree (cyc/deg) plotted against days. In control animals, during 7 days of MD (control+MD), visual acuity of the open eye significantly increased. In contrast, in PT-animals, MD failed to induce improvement (PT+MD). Treatment with the antiinflammatory drug ibuprofen $(\mathrm{PT}+\mathrm{MD}+\mathrm{Ibu})$ but not vehicle $(\mathrm{PT}+\mathrm{MD}+\mathrm{NaCl})$ rescued this enhancement to control values (control+MD). Ibuprofen treatment itself did not affect visual acuity of control mice (control+Ibu). Modified from Greifzu et al. (2011). 

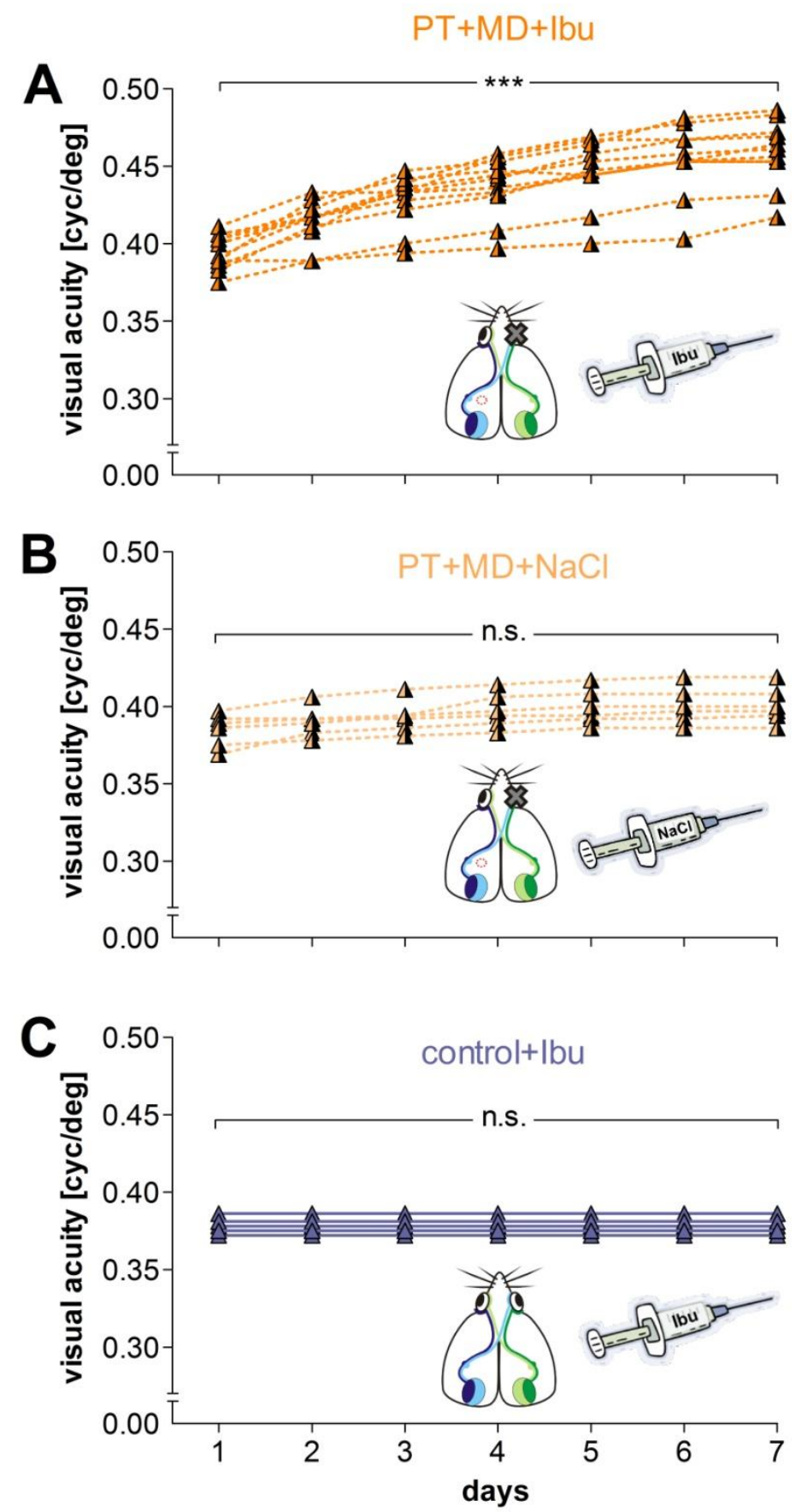

Figure 40: The visual acuity of the open eye of PT-mice after MD increased significantly with ibuprofen (Ibu) treatment $(\mathrm{A})$, but not with vehicle $(\mathrm{NaCl})$ treatment $(\mathrm{B})$. $(\mathrm{C})$ Ibuprofen treatment itself had no effect the visual acuity of control mice. Each line represents one animal.

The gain on baseline analysis also showed that the increase in visual acuity in PT-mice treated with ibuprofen was with $16.2 \pm 1.2 \%(\triangleq 0.06 \pm 0.005 \mathrm{cyc} / \mathrm{deg})$ after MD significantly higher than in untreated PT-mice with $\mathrm{MD}\left(\mathrm{F}_{1,19}=50.81, \mathrm{P}<0.001\right.$, ANOVA $)$ and not different to control mice $\left(\mathrm{F}_{1,20}=0.03, \mathrm{P}>0.05\right.$, ANOVA) (Figure 41). 


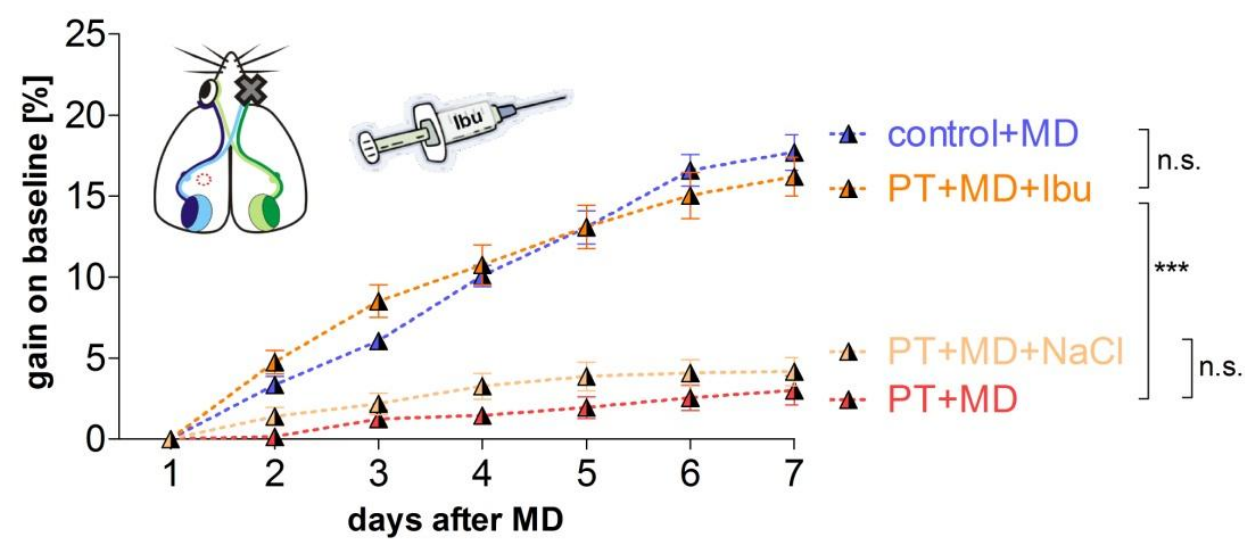

Figure 41: The percentage gain on baseline also shows that after MD the visual acuity of the open eye of PT-mice treated with ibuprofen (Ibu) increased significantly more than untreated PT-mice. Vehicle treatment with $\mathrm{NaCl}$ did not restore enhancement of visual acuity after MD.

Ibuprofen treatment also restored the enhancement of contrast sensitivity after MD in PTanimals. In ibuprofen treated PT animals, MD of the right eye led to a significant enhancement of contrast sensitivity ( $P<0.001$, Bonferroni-adjusted t-test; $n=12$ ): at a spatial frequency of $0.064 \mathrm{cyc} / \mathrm{deg}$, contrast sensitivity was $16.7 \pm 0.48$ ( $\triangleq 6 \%$ contrast $)$ on day 1 and increased to $23.3 \pm 1.12$ ( $4 \%$ contrast) after 7 days of MD (Figure $43 \mathrm{~A}$ ). The contrast sensitivity at all measured frequencies was significantly higher compared to PT-mice without treatment after MD (Figure 42). In contrast, in vehicle treated PT animals, contrast sensitivity did not increase significantly after MD $(P>0.05$, Bonferroni-adjusted t-test, $n=6)$ : contrast values at $0.064 \mathrm{cyc} / \mathrm{deg}$ were $13.0 \pm 0.67$ (^ $8 \%$ contrast) on day 1 and $15.4 \pm 1.05(\hat{=} 7 \%$ contrast) on day 7 (Figure 43 B). Contrast sensitivity at all measured frequencies was not significantly different to PT-mice without treatment after MD (Figure 42). Ibuprofen treatment itself had no effect on the contrast sensitivity of control mice (at $0.064 \mathrm{cyc} / \mathrm{deg}$, day 1: $15.7 \pm 0.42 \mathrm{cyc} / \mathrm{deg}$; day $7: 15.7 \pm 0.42 \mathrm{cyc} / \mathrm{deg}, \mathrm{P}>0.05$, Bonferroni-adjusted t-test, $\mathrm{n}=6$ ) (Figure $43 \mathrm{C}$ ). Average contrast values on days 1 and 7 after MD at all measured spatial frequencies and P-values (Bonferroni-adjusted t-test) are listed in Table 5.

Concluding, ibuprofen treatment completely rescued the improvement of both visual acuity and contrast sensitivity of the open eye after MD in PT-animals. 


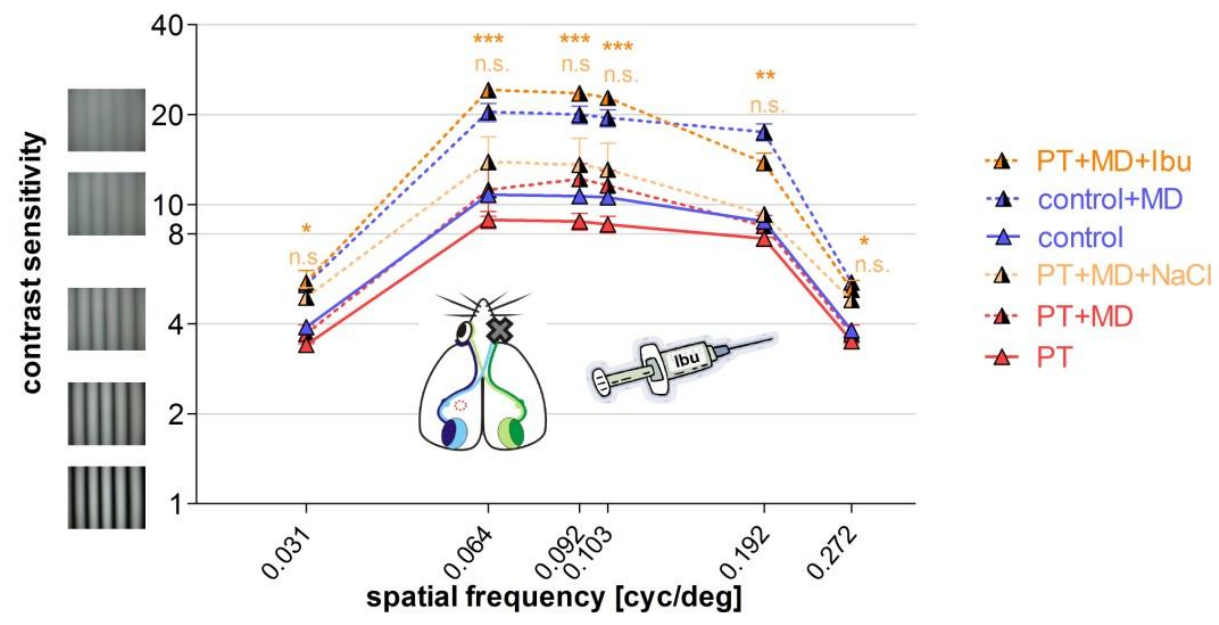

Figure 42: Ibuprofen-, but not vehicle treatment restored visual acuity increase after MD in PTmice. Contrast sensitivity was measured at 6 different spatial frequencies. Illustrated values are from day 7 (after MD in deprived animals). Contrast sensitivity of the open eye in PT-mice with ibuprofen (Ibu) treatment increased significantly at all measured frequencies after MD. In PT-mice with vehicle treatment $(\mathrm{NaCl})$ contrast sensitivity of the open eye did not increase significantly after MD. Indicated significances are in comparison to the $\mathrm{PT}+\mathrm{MD}$ group. 

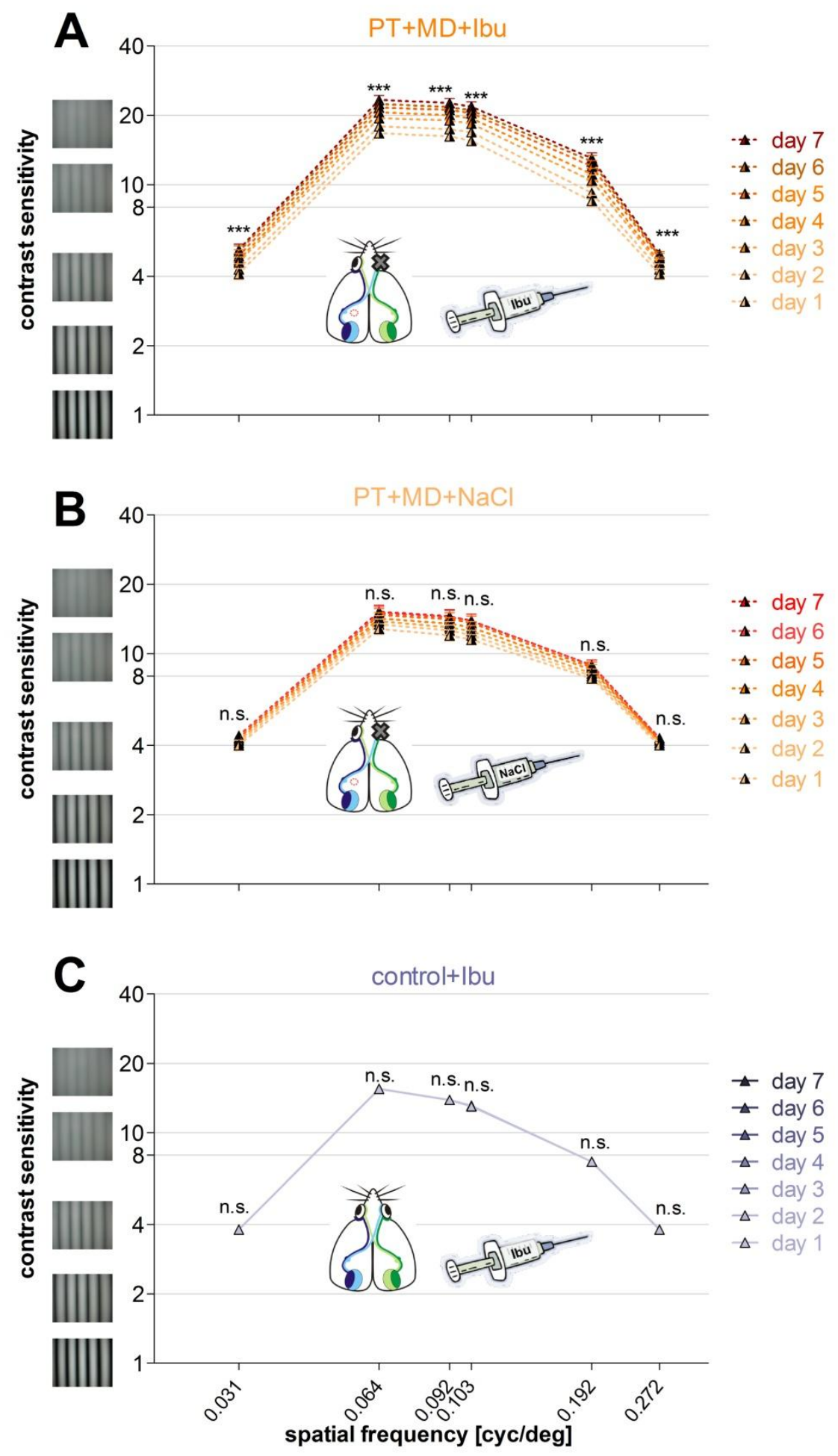

Figure 43: Contrast sensitivity of the open eye after MD in PT-lesioned mice increased significantly after ibuprofen (Ibu) treatment (A), but not after vehicle ( $\mathrm{NaCl}$ ) treatment $(\mathrm{B})$. (C) In control mice without MD ibuprofen treatment itself did not affect contrast sensitivity. 
Table 5: Contrast sensitivity of the open eye increased significantly in ibuprofen (Ibu) treated but not in vehicle $(\mathbf{N a C l})$ treated PT-animals after MD. Average contrast values \pm S.E.M. on day 1 and day 7 after $\mathrm{MD}$ and $\mathrm{NaCl}$ or ibuprofen treatment, respectively and statistical significance for the increase over these days (Bonferroni-adjusted t-test).

\begin{tabular}{|c|c|c|c|}
\hline Frequencies [cyc/deg] & Contrast sensitivity day 1 & Contrast sensitivity day 7 & $\mathrm{P}$ \\
\hline $\mathrm{PT}+\mathrm{MD}+\mathrm{NaCl}$ & & & \\
\hline 0.031 & $4.1 \pm 0.21$ & $4.4 \pm 0.19$ & $>0.05$ \\
0.064 & $13.0 \pm 0.67$ & $15.4 \pm 1.05$ & $>0.05$ \\
0.092 & $12.1 \pm 0.68$ & $14.7 \pm 1.04$ & $>0.05$ \\
0.103 & $11.7 \pm 0.61$ & $14.1 \pm 0.99$ & $>0.05$ \\
0.192 & $7.9 \pm 0.38$ & $9.0 \pm 0.49$ & $>0.05$ \\
0.272 & $4.0 \pm 0.15$ & $4.4 \pm 0.15$ & $>0.05$ \\
\hline $\mathrm{PT}+\mathrm{MD}+$ Ibuprofen & & & $<0.001$ \\
0.031 & $4.1 \pm 0.23$ & $5.2 \pm 0.32$ & $<0.001$ \\
0.064 & $16.7 \pm 0.48$ & $23.3 \pm 1.12$ & $<0.001$ \\
0.092 & $16.2 \pm 0.53$ & $22.6 \pm 1.10$ & $<0.001$ \\
0.103 & $15.5 \pm 0.54$ & $21.8 \pm 1.06$ & $<0.001$ \\
0.192 & $8.5 \pm 0.51$ & $13.0 \pm 0.77$ & $<0.001$ \\
0.272 & $4.1 \pm 0.23$ & $5.0 \pm 0.23$ & \\
\hline
\end{tabular}

MD of the left eye. Ibuprofen treatment also restored visual acuity improvement of PTanimals after left eye MD: visual acuity of the open eye increased significantly from $0.38 \pm 0.005 \mathrm{cyc} / \mathrm{deg}$ (day 1$)$ to $0.46 \pm 0.009 \mathrm{cyc} / \mathrm{deg}(\mathrm{P}<0.01$, Bonferroni-adjusted t-test, $\mathrm{n}=6)$. This increase was indistinguishable from control animals with $\mathrm{MD}\left(\mathrm{F}_{1,14}=0.33\right.$, $\mathrm{P}>0.05$, ANOVA $)$ and PT-animals with ibuprofen treatment and right eye $\mathrm{MD}\left(\mathrm{F}_{1,16}=1.08\right.$, $\mathrm{P}>0.05$, ANOVA).

However, the enhancement of contrast sensitivity after MD in PT-animals with ibuprofen treatment was not completely restored. The increase in visual acuity was just significant at the frequency of $0.031 \mathrm{cyc} / \mathrm{deg}$ and $0.272 \mathrm{cyc} / \mathrm{deg}(\mathrm{P}<0.05$, Bonferroni-adjusted t-test; $\mathrm{n}=6)$.

Concluding, ibuprofen could restore enhancement of visual acuity in PT-mice also after left eye MD. This result is particularly promising, because the hemisphere which is responsible for the increase in visual acuity is the lesioned hemisphere (ipsilateral to the deprived left eye) (Prusky et al. 2006).

\section{Late MD restored enhancement of vision in PT-mice}

To analyze whether the diminished plasticity after a PT-lesion was an acute effect or a longerlasting modification of neuronal circuits, we next introduced a delay of 1 or 2 weeks between the lesion and MD (deprivation of the right eye). When MD was started 1 week after PT, 
visual acuity improved only slightly (day 1: $0.40 \pm 0.01 \mathrm{cyc} / \mathrm{deg}$; day 7: $0.43 \pm 0.01 \mathrm{cyc} / \mathrm{deg}$; $\mathrm{P}<0.05$, Bonferroni-adjusted t-test, $\mathrm{n}=5$ ) (Figure 44, Figure $45 \mathrm{~A}$ ), while the increase was significant after a two-week delay (day 1: $0.39 \pm 0.00 \mathrm{cyc} / \mathrm{deg}$; day 7: $0.45 \pm 0.01 \mathrm{cyc} / \mathrm{deg}$; $\mathrm{P}<0.001$, Bonferroni-adjusted t-test, $\mathrm{n}=11$ ) (Figure 44, Figure $45 \mathrm{~B}$ ). This improvement was significantly different from mice with $\mathrm{MD}$ directly after the PT-lesion $\left(\mathrm{F}_{1,18}=19.46\right.$, $\mathrm{P}<0.001$, ANOVA $)$ and indistinguishable from control animals with $\mathrm{MD}\left(\mathrm{F}_{1,19}=1.72\right.$, $\mathrm{P}>0.05$, ANOVA).

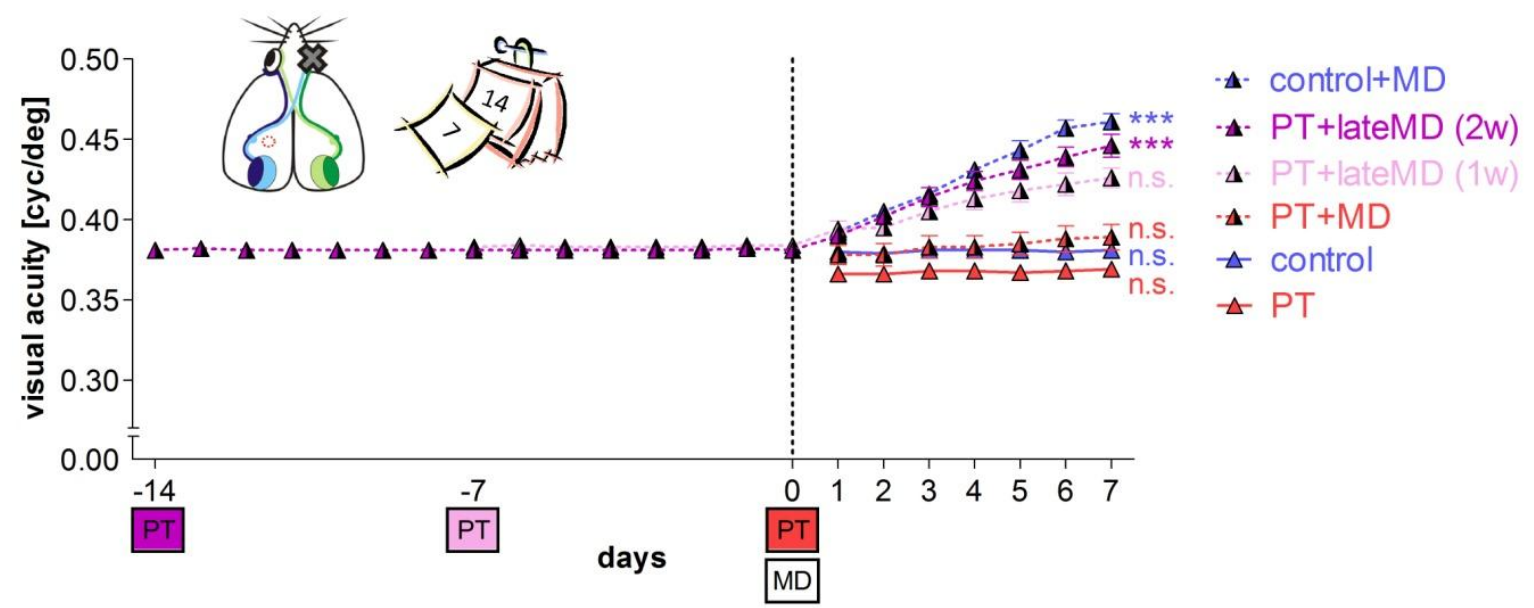

Figure 44: Late MD rescued visual acuity improvement in PT-mice. In control animals, during 7 days of MD (control+MD), visual acuity of open eyes significantly increased. In contrast, in PTanimals, MD failed to induce improvement (PT+MD). After a 1 week delay between PT and MD (PT+ lateMD (1w)), visual acuity increased only slightly. After a 2 week delay (PT+ lateMD (2w)) visual acuity was significantly enhanced compared to animals with an MD directly after a lesion (PT+MD), and values were indistinguishable from controls with MD (control+MD). Modified from Greifzu et al. (2011). 

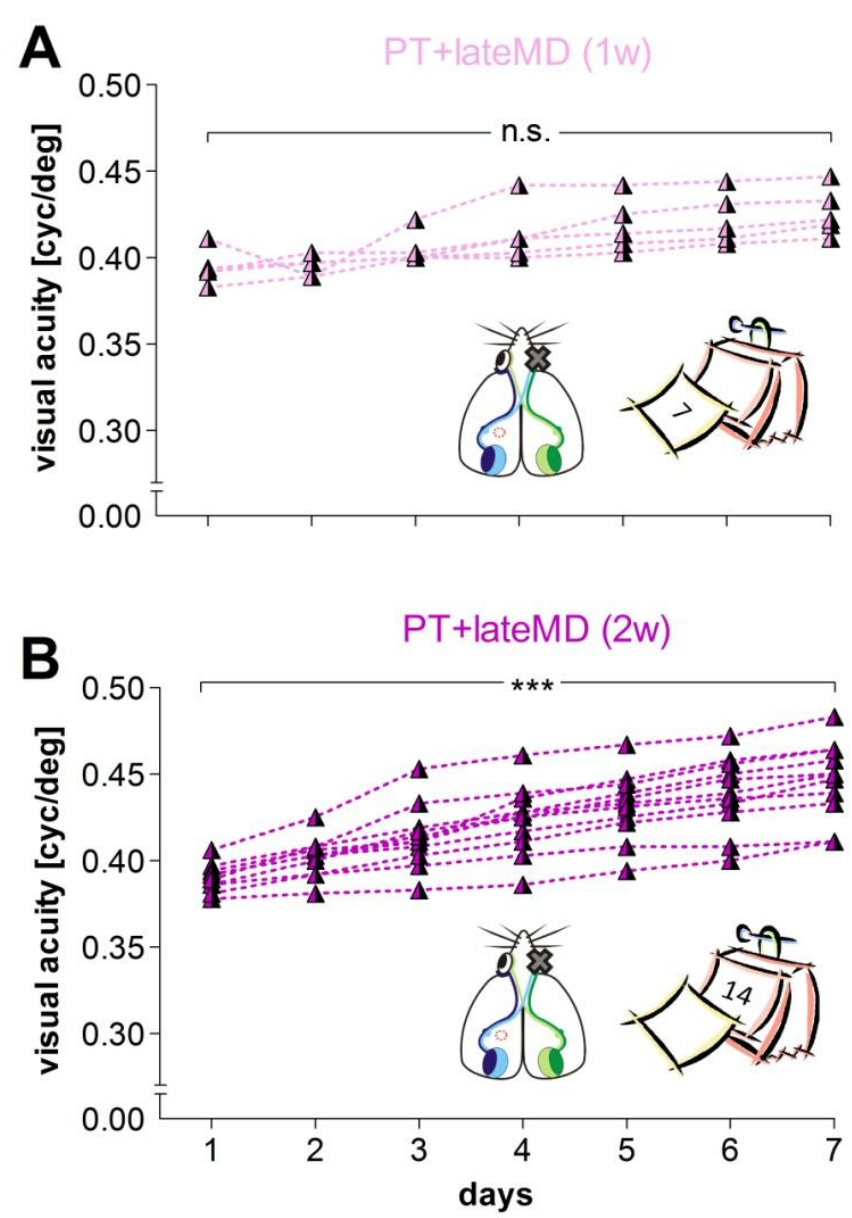

Figure 45: Visual acuity in the late MD paradigm. (A) If the MD was performed 1 week after PT visual acuity of the open eye increased only slightly but not significantly after MD. (B) After a 2 week delay visual acuity of the open eye increased significantly after MD. Each line represents one animal.

The gain on baseline analysis revealed the same result: the increase in visual acuity was with $14.3 \pm 1.5 \%(\hat{=} 0.06 \pm 0.01 \mathrm{cyc} / \mathrm{deg})$ after a 2 week delay between PT and MD significantly higher than without delay $\left(\mathrm{F}_{1,18}=42.20, \mathrm{P}<0.001\right.$, ANOVA $)$ and was similar to control-mice with $\mathrm{MD}\left(\mathrm{F}_{1,19}=3.03, \mathrm{P}>0.05\right.$, ANOVA) (Figure 46). After a 1 week delay (increase: $6.0 \pm 1.7 \% \triangleq 0.2 \pm 0.01 \mathrm{cyc} / \mathrm{deg})$ the increase was also significantly higher than with no delay $\left(\mathrm{F}_{1,14}=7.76, \mathrm{P}<0.05\right.$, ANOVA $)$. 


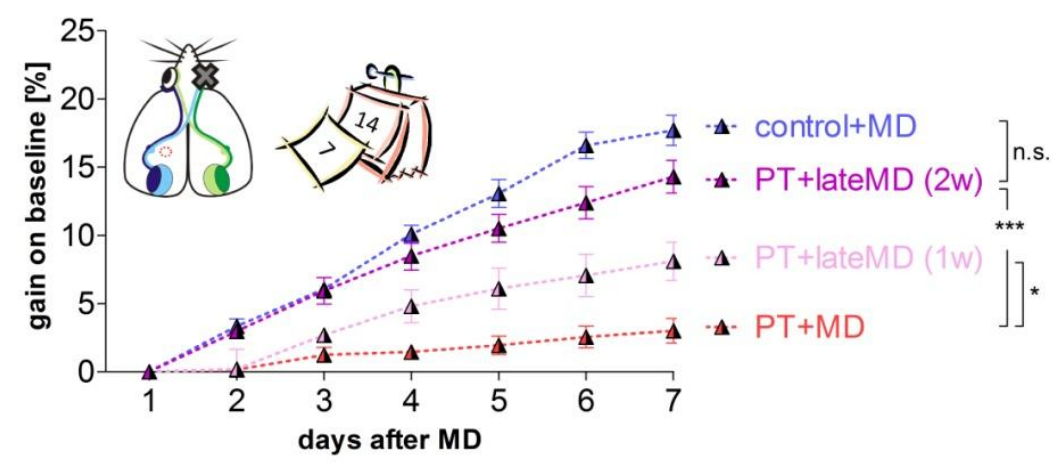

Figure 46: The percentage gain on baseline also shows that late MD rescued visual acuity improvement in PT-mice. The visual acuity increased significantly stronger after a 2 week delay between PT and MD than with no delay or just 1 week delay and was similar to control-mice values.

Contrast sensitivity also significantly increased in PT-animals deprived 2 weeks after a PTlesion: at a spatial frequency of $0.064 \mathrm{cyc} / \mathrm{deg}$, contrast sensitivity was $13.5 \pm 0.51$ ( $\triangleq 8 \%$ contrast) on day 1 and increased to $21.3 \pm 1.80$ ( $\triangleq 5 \%$ contrast $)$ after 7 days of $\mathrm{MD}(\mathrm{P}<0.01$, Bonferroni-adjusted t-test; $n=11$ ) (Figure $48 \mathrm{~B}$ ). The contrast sensitivity at all measured frequencies was significantly higher compared to PT-mice without a delay until MD (Figure 47). PT-animals deprived 1 week after the PT-lesion induction showed no significant enhancement of contrast sensitivity: contrast values on day $1(11.0 \pm 1.27, \triangleq 9 \%$ contrast at 0.064 cyc/deg, $\mathrm{n}=5)$ were not significantly different from values on day $7(14.7 \pm 1.40$, $\hat{=} 7 \%$ contrast, $\mathrm{n}=5 ; \mathrm{P}>0.05$, Bonferroni-adjusted t-test) (Figure $48 \mathrm{~A}$ ). The contrast sensitivity at all measured frequencies was not significantly different compared to PT-mice without a delay until MD (Figure 47). Average contrast values on days 1 and 7 after MD at all measured spatial frequencies and P-values (Bonferroni-adjusted t-test) are listed in Table 6.

Taken together, our data show that a delay of 2 weeks between lesion induction and MD was sufficient to completely restore the enhancement of both visual acuity and contrast sensitivity, indicating that the reduced sensory learning was a transient phenomenon. 


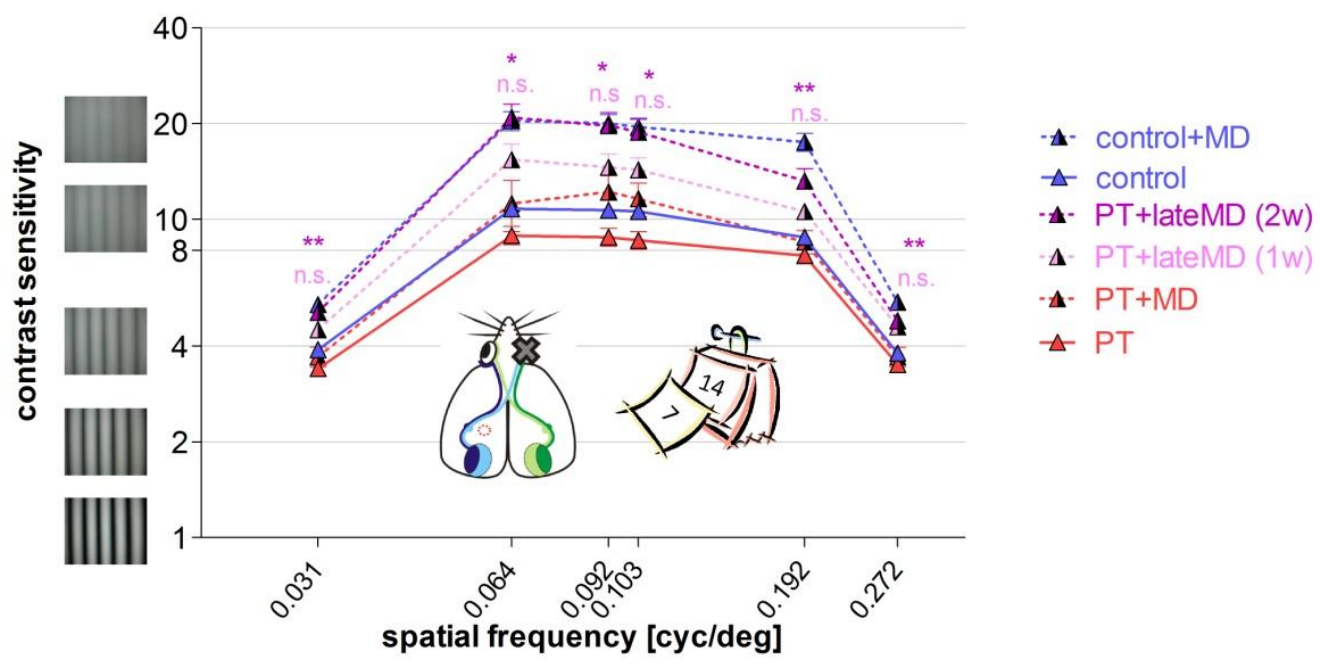

Figure 47: A 2 week delay between PT-lesion and MD, but not a 1 week delay restored visual acuity increase after MD in PT-mice. Contrast sensitivity was measured at 6 different spatial frequencies. Illustrated values are from day 7 (after MD in deprived animals). Contrast sensitivity of the open eye in PT-mice with a 2 week delay increased significantly at all measured frequencies after $\mathrm{MD}$, and was significantly different to PT-mice without delay of MD. A 1 week delay was not enough to restore contrast sensitivity increase. Indicated significance-levels are in comparison to the PT+MD group. 

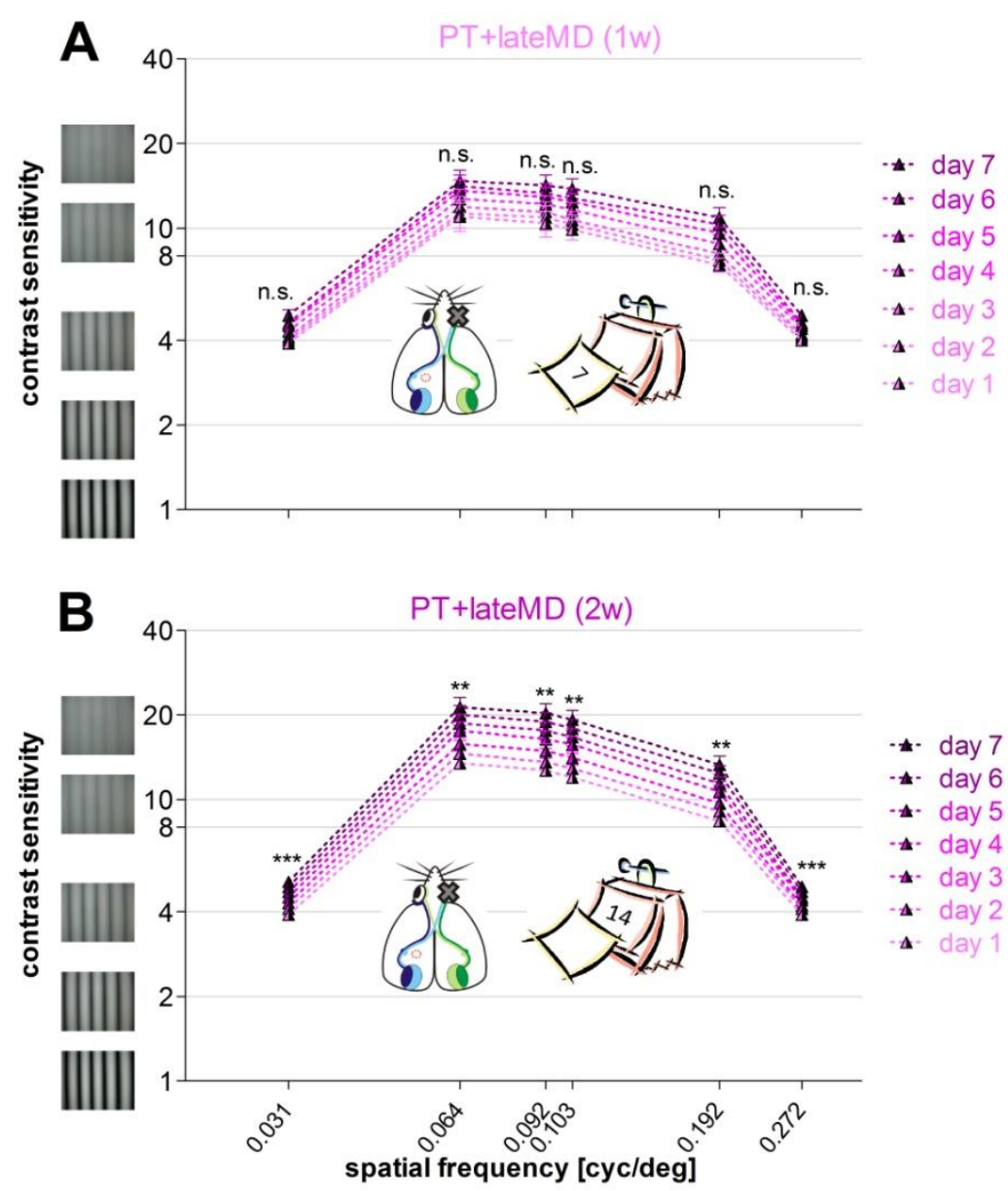

Figure 48: Contrast sensitivity after MD in the late MD paradigm. (A) After a delay of 1 week between PT and MD, contrast sensitivity increased only slightly but not significantly. (B) After a 2 week delay contrast sensitivity of the open eye increased significantly at all measured frequencies after MD. Shown significance-levels indicate comparison between day 1 and 7.

Table 6: Contrast sensitivity of the open eye significantly increased in PT-animals deprived 2 weeks but not 1 week after a lesion. Average contrast values \pm S.E.M. on day 1 and day 7 after MD and statistical significance for the increase over these days (Bonferroni-adjusted t-test).

\begin{tabular}{|r|c|c|c|}
\hline Frequencies [cyc/deg] & Contrast sensitivity day 1 & Contrast sensitivity day 7 & $\mathrm{P}$ \\
\hline $\mathrm{PT}+_{\text {late }} \mathrm{MD}(1 \mathrm{w})$ & & & \\
0.031 & $3.0 \pm 0.03$ & $4.9 \pm 0.23$ & $>0.05$ \\
0.064 & $11.0 \pm 1.27$ & $14.7 \pm 1.40$ & $>0.05$ \\
0.092 & $10.4 \pm 1.09$ & $14.2 \pm 1.27$ & $>0.05$ \\
0.103 & $9.9 \pm 0.82$ & $13.8 \pm 1.18$ & $>0.05$ \\
0.192 & $7.4 \pm 0.42$ & $10.9 \pm 0.93$ & $>0.05$ \\
0.272 & $4.0 \pm 0.05$ & $4.9 \pm 0.12$ & $>0.05$ \\
\hline $\mathrm{PT}+{ }_{\text {late }} \mathrm{MD}(2 \mathrm{w})$ & & & \\
0.031 & $3.9 \pm 0.10$ & $5.1 \pm 0.22$ & $<0.001$ \\
0.064 & $13.5 \pm 0.51$ & $21.3 \pm 1.80$ & $<0.01$ \\
0.092 & $12.7 \pm 0.48$ & $20.3 \pm 1.66$ & $<0.01$ \\
0.103 & $12.0 \pm 0.43$ & $19.2 \pm 1.56$ & $<0.01$ \\
0.192 & $8.4 \pm 0.31$ & $13.3 \pm 1.00$ & $<0.01$ \\
0.272 & $3.9 \pm 0.07$ & $4.9 \pm 0.19$ & $<0.001$ \\
\hline
\end{tabular}




\subsubsection{Ibuprofen and late MD did not restore OD-plasticity}

\section{Ibuprofen did not restore OD-plasticity in PT-mice}

To test whether anti-inflammatory treatment with ibuprofen could also restore OD-plasticity after a PT-lesion, visual cortical activity was imaged directly after the behavioral measurements. In ibuprofen treated PT-animals, MD could not induce a significant OD-shift: the binocular visual cortex remained dominated by input from the contralateral (deprived) eye. Activity patches induced by stimulation of the contralateral (deprived) eye were still darker than those of the ipsilateral (open) eye (Figure 49 A). The histogram of ODIs was still centered right of zero and the two-dimensional OD-map displayed warm red colors. The ODI was positive (average ODI: $0.14 \pm 0.03, \mathrm{n}=10$ ) and not significantly different from PT-mice without MD (P > 0.05, Bonferroni-adjusted t-test) (Figure $50 \mathrm{~A})$. A control group of vehicle $(\mathrm{NaCl})$ treated PT-mice with MD also showed no OD-shift: Activity patches induced by stimulation of the contralateral (deprived) eye were still darker than those of the ipsilateral (open) eye (Figure 49 B). The histogram of ODIs was still centered right of zero and the twodimensional OD-map displayed warm red colors. The ODI $(0.16 \pm 0.02, n=6)$ was neither significantly different to PT-mice with MD and ibuprofen treatment $(\mathrm{P}>0.05$, Bonferroniadjusted t-test), nor to PT-mice without MD ( $\mathrm{P}>0.05$, Bonferroni-adjusted t-test) (Figure $50 \mathrm{~A}$ ). The ibuprofen treatment itself had no effect on the OD (Figure $49 \mathrm{C}$ ): the ODI of control mice without MD treated with ibuprofen $(0.21 \pm 0.02, n=6)$ was not significantly different to control mice without treatment $(\mathrm{P}>0.05$, Bonferroni-adjusted t-test).

Analyses of maximum response strength also revealed that cortical activation was indistinguishable from untreated PT-animals: cortical activation after deprived-eye stimulation remained higher than after open eye stimulation, irrespective of whether animals were injected with ibuprofen (deprived/open: $2.52 \pm 0.20 / 1.93 \pm 0.12 ; \mathrm{P}<0.001$, t-test) or vehicle (deprived/open: $2.22 \pm 0.10 / 1.63 \pm 0.02 ; \mathrm{P}<0.01$, t-test) (Figure $50 \mathrm{~B}$ ). In control animals, ibuprofen treatment also had no effect on the maximum response strength compared to untreated control mice (contra/ipsi: $2.36 \pm 0.21 / 1.56 \pm 0.12, \mathrm{n}=6 ; \mathrm{P}>0.05$, t-test). 

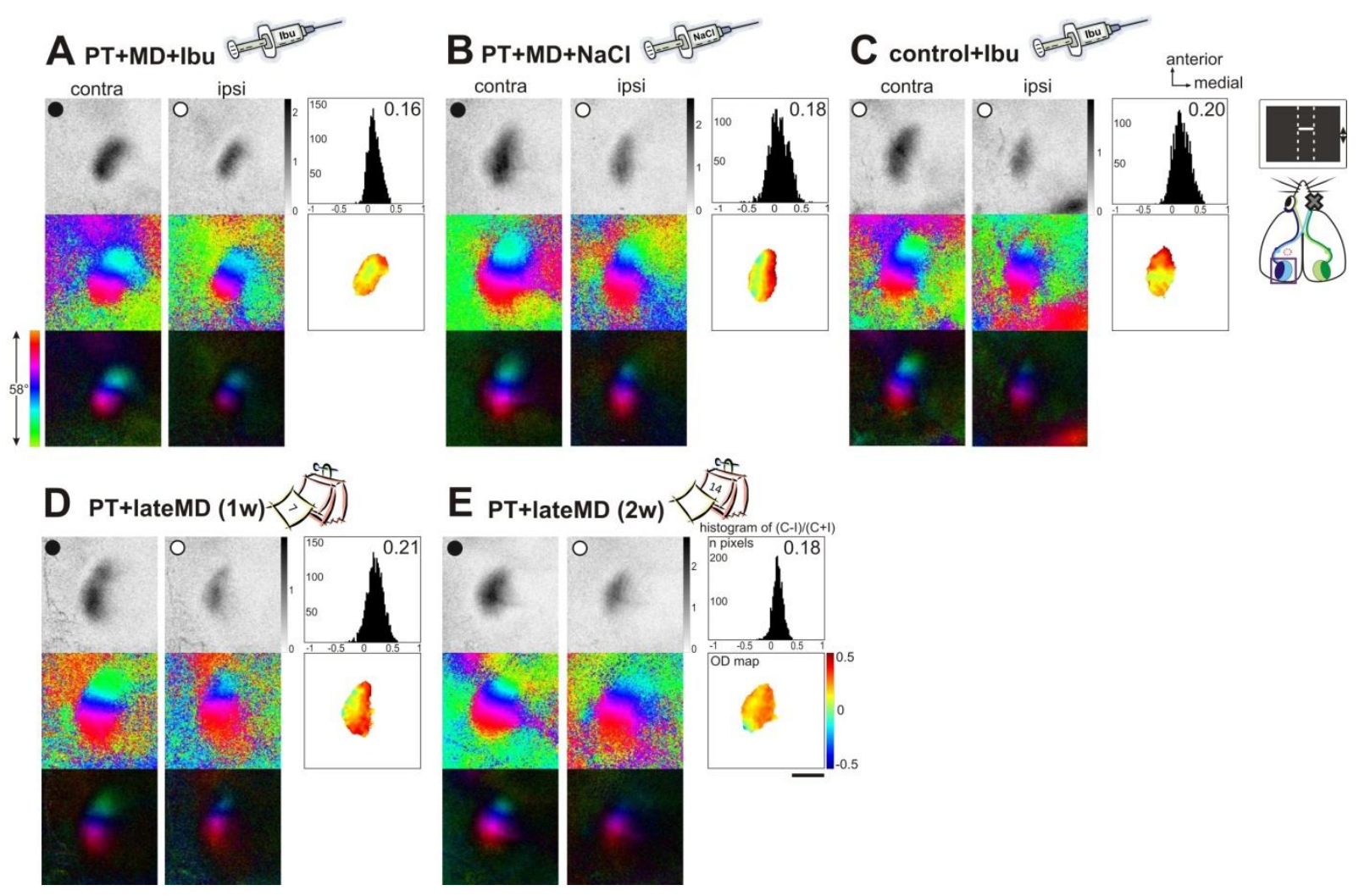

Figure 49: Ibuprofen and late MD did not restore OD-plasticity after a PT-lesion. Optically imaged maps of responses after stimulation of the contra- and ipsilateral (ipsi) eye in the binocular region of mouse visual cortex. Both gray-scale coded response magnitude maps and their quantification (top), and color-coded phase and polar maps of retinotopy (middle, bottom) are illustrated. The magnitude of the optical responses is illustrated as fractional change in reflection $\times 10^{4}$. For each experiment, the histogram of OD-scores, the average OD-index (ODI), and the corresponding two-dimensional OD-maps are included (ODI values are color-coded: blue represents negative, red positive values). Black filled circles on the response magnitude maps indicate the deprived eye, unfilled circles non-deprived eyes. PT-animals were still dominated by contralateral eye treatment, even after ibuprofen (A) treatment: activity patches evoked by stimulation of the contralateral eye were darker than those after stimulation of the ipsilateral eye, two-dimensional OD-maps display warm colors, and the average ODI was positive, indicating contralateral dominance. The same was true for the vehicle $(\mathrm{NaCl})$ treated group $(\mathrm{B})$ and control mice with ibuprofen treatment $(\mathrm{C})$. Even after a 1 week $(1 \mathrm{w})$ (D) or 2 weeks $(2 \mathrm{w})(\mathrm{E})$ delay between PT and MD the contralateral dominance persisted and no OD-shift occurred. Scale bar: $1 \mathrm{~mm}$. 


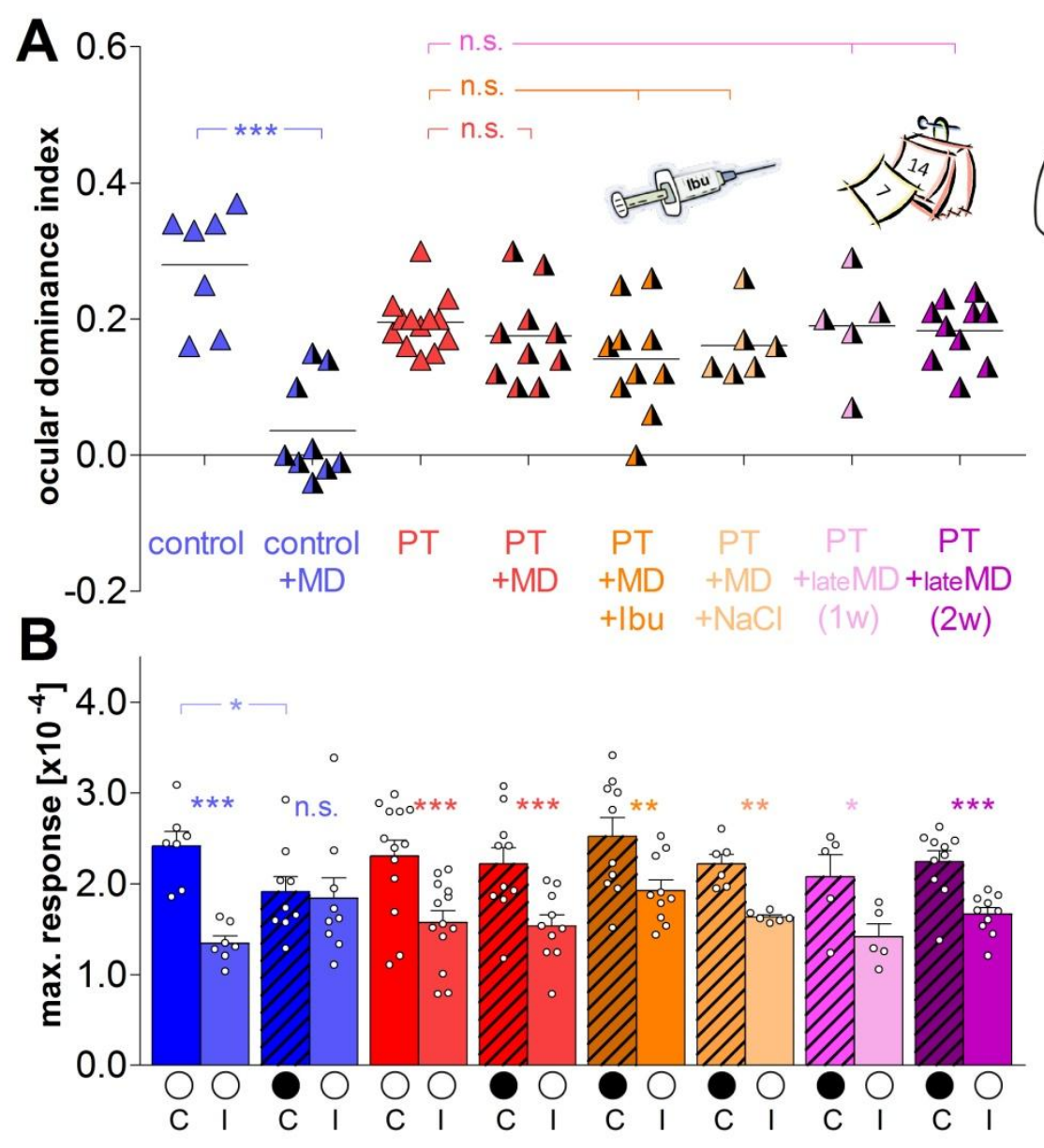

Figure 50: Ibuprofen and late MD did not restore OD-plasticity after a PT-lesion (PT). (A) ODindices of the left (lesioned) hemisphere after MD of the contralateral eye (see inset). Symbols represent ODI values of individuals; means are marked by horizontal lines. In control animals, MD induced a significant reduction of ODIs (control+MD). In contrast, in PT-animals, ODIs were not changed after MD, not even after anti-inflammatory treatment with ibuprofen (Ibu) or a 1 week $(1 \mathrm{w})$ or 2 week (2w) delay between PT and MD (B) Maximum response magnitude in V1 expressed as fractional change in light reflectance elicited by stimulation of the contralateral (C) or ipsilateral (I) eye. Filled circles represent deprived eyes, unfilled circles non-deprived eyes. In control animals, cortical activation after visual stimulation of the contralateral eye was significantly higher than after ipsilateral eye stimulation, reflecting the dominance of the contralateral eye. After MD of the contralateral eye, response strengths of the two eyes were no longer significantly different. In contrast, after MD in PT-animals, the response strengths of both eyes remained significantly different, even after ibuprofen or vehicle $(+\mathrm{NaCl})$ treatment or late MD. Modified from Greifzu et al. (2011).

\section{Late MD did not restore OD-plasticity in PT-mice}

We also imaged visual cortical activity of animals with late MD to test whether OD-plasticity was restored. After both, a 1 week or a 2 week delay, MD did not induce a significant ODshift: the binocular visual cortex remained dominated by input from the contralateral (deprived) eye. Activity patches induced by stimulation of the contralateral (deprived) eye 
were still darker than those of the ipsilateral (open) eye (Figure $49 \mathrm{D}, \mathrm{E}$ ). The histogram of ODIs was still centered right of zero and the two-dimensional OD-map displayed warm red colors. The ODI of both groups was positive ( 1 week: $0.19 \pm 0.04, \mathrm{n}=5 ; 2$ weeks: $0.18 \pm 0.01, \mathrm{n}=10$ ) and not significantly different from each other and from PT-mice without MD (for all P > 0.05, Bonferroni-adjusted t-test) (Figure $50 \mathrm{~A}$ ).

Maximum response strength after deprived eye stimulation also remained significantly higher than after open eye stimulation ( 1 week: deprived/open: $2.08 \pm 0.24 / 1.42 \pm 0.14$; $\mathrm{P}<0.05$, $\mathrm{t}$ test; 2 weeks: deprived/open: $2.24 \pm 0.12 / 1.67 \pm 0.07$; $\mathrm{P}<0.001$, t-test) (Figure $50 \mathrm{~B}$ ). Thus, OD-plasticity was not restored by a 1 week or 2 week delay between lesion induction and MD.

Altogether our results show that sensory learning and OD-plasticity were diminished after a PT-lesion, but at least sensory learning could be restored by an anti-inflammatory treatment and a 2 week delay between PT-lesion and MD.

\subsection{Overview of OD-plasticity in both hemispheres}

For an overview, the ODIs and response magnitudes for all the experimental groups mentioned in the results before are compiled here in one graph. Shown are the ODIs and the response magnitudes of both eyes for groups without MD and after MD of the contralateral or ipsilateral eye respectively, shown for the lesioned hemisphere (Figure 51) and for the nonlesioned hemisphere (Figure 52).

Lesioned hemisphere (Figure 51). In control mice a significant OD-shift towards the open eye could be induced by contralateral eye $M D$. This shift was mediated by a reduction in deprived eye responses. In contrast to that, in PT-mice no OD-plasticity could be induced by contralateral eye MD, not even after different treatment regimes (anti-inflammatory treatment with ibuprofen, late MD). Nevertheless, a significant OD-shift towards the open eye could be induced by an ipsilateral eye MD in all in PT-groups. But this was not the case in control mice. Concluding, in the lesioned hemisphere of PT-mice, no OD-plasticity could be observed after contralateral eye MD, but a significant OD-shift could still be induced after ipsilateral eye MD.

Non-lesioned hemisphere (Figure 52). MD of the contralateral eye induced a significant ODshift towards the open eye in control as well as in PT-mice, but not after ibuprofen treatment 
and a delay of 2 weeks between PT-lesion and MD. After ipsilateral eye MD no significant OD-shift was induced in control mice, but in PT-mice (except for lateMD (1w)).

Consequently, a PT-lesion selectively disturbed OD-plasticity in V1 of the lesioned hemisphere while OD-plasticity was still present in the non-lesioned hemisphere.

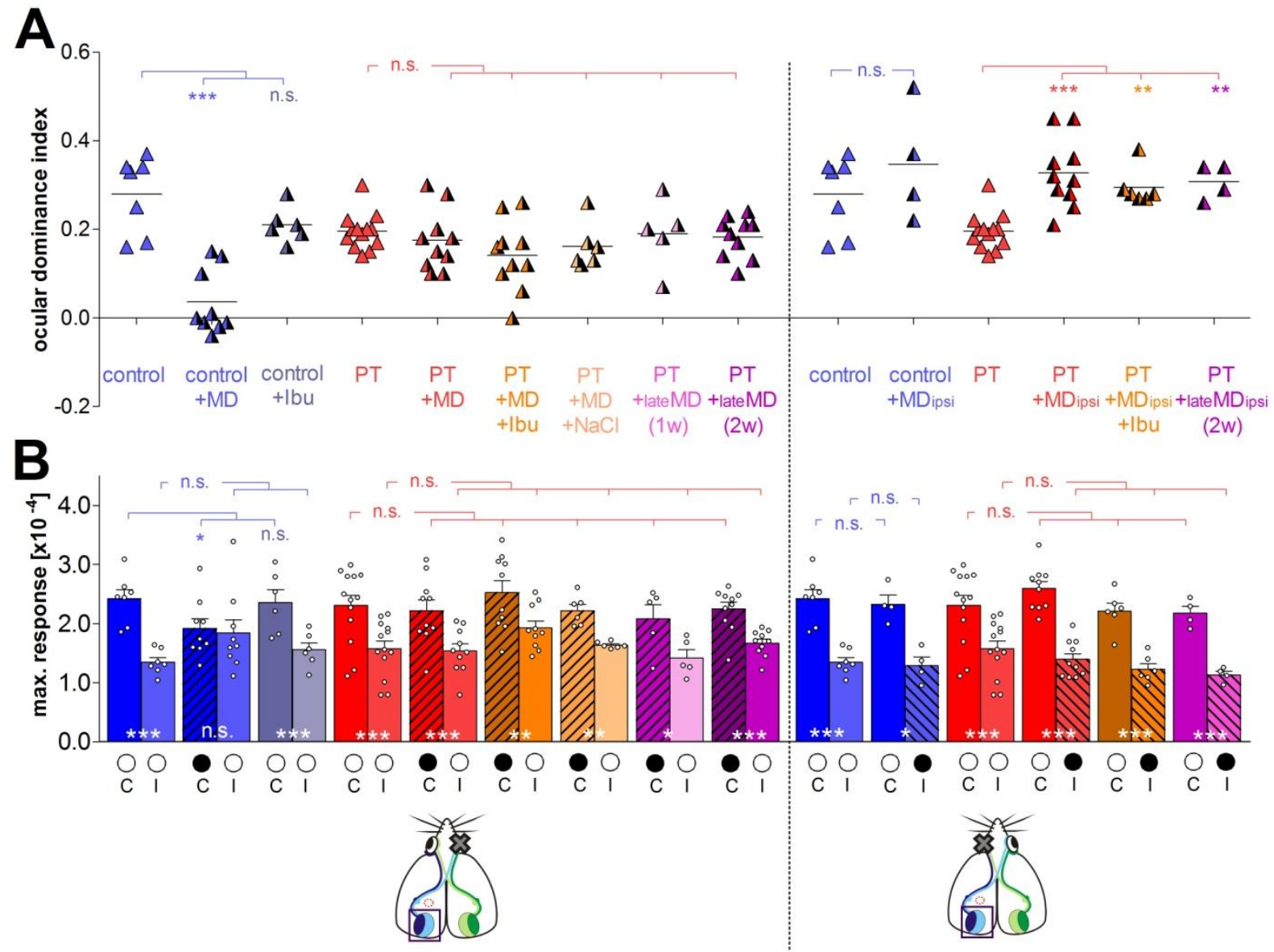

Figure 51: Lesioned hemisphere: No OD-plasticity in PT-mice after contralateral eye MD, but after ipsilateral eye MD. (A) ODIs after deprivation of either the contralateral (MD) or the ipsilateral eye (MDipsi) (see inset below). Symbols represent ODI values of individuals; means are marked by horizontal lines. (B) Shown are response magnitudes after stimulation of the contra (C) or ipsilateral (I) eye for the respective groups. Filled circles represent deprived eyes, unfilled circles open eyes. Marked in white on the bars is the statistical comparison between response magnitude after contraand ipsilateral eye stimulation in the respective group. After contralateral eye $M D$ a significant ODshift could be induced in control animals. This shift was mediated by a decrease in closed eye responses. In contrast to that, in PT-mice no OD-plasticity could be induced, not even after different treatment regimes (anti-inflammatory treatment with ibuprofen (Ibu), late MD). Nevertheless, a significant OD-shift in PT-mice could be induced by an ipsilateral eye MD. But it did not induce a significant shift in control mice. 


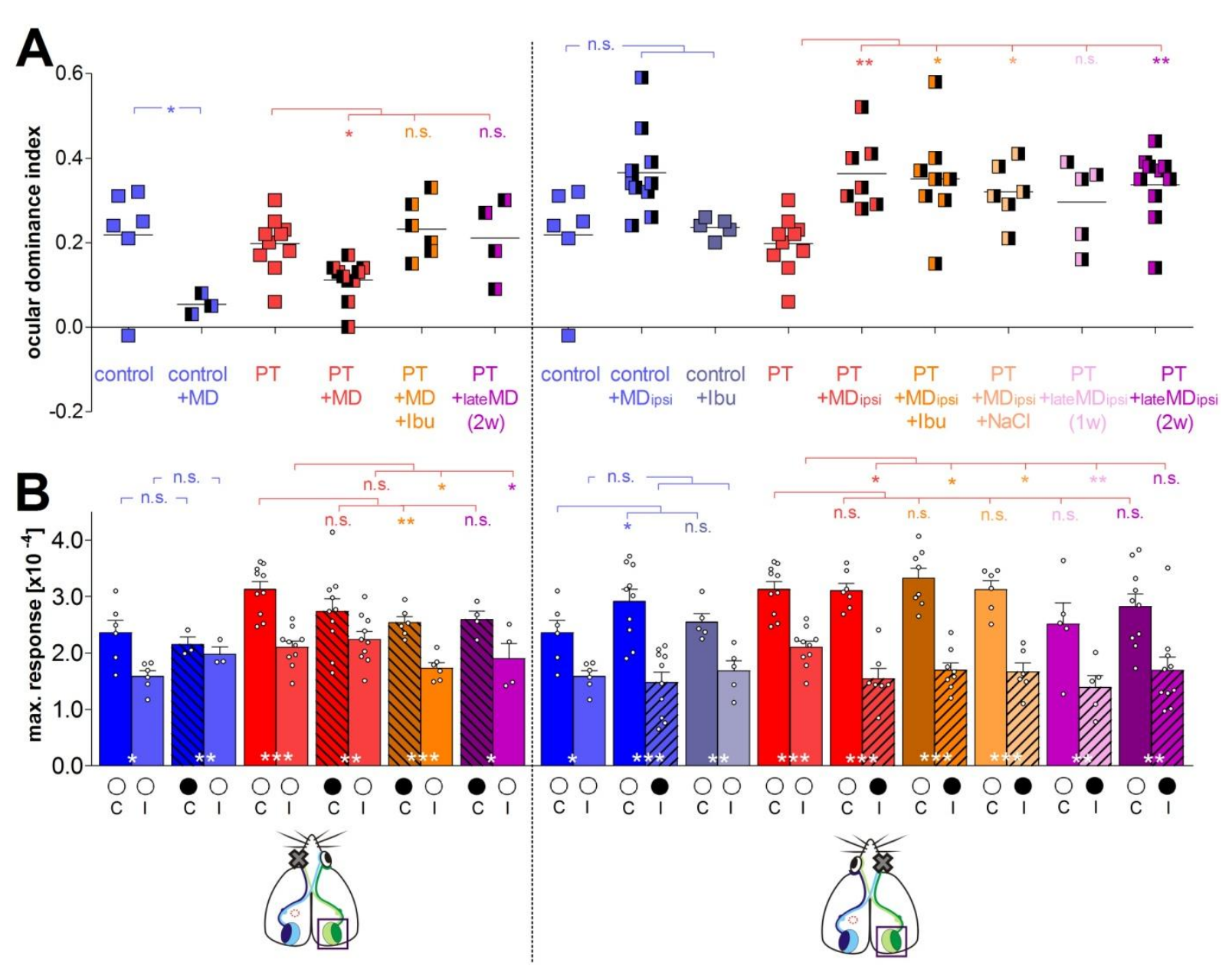

Figure 52: Non-lesioned hemisphere: OD-plasticity in PT-mice after contralateral and ipsilateral eye MD. (A) ODIs after deprivation of either the contralateral (MD) or the ipsilateral eye (MDipsi) (see inset below). Symbols represent ODI values of individuals; means are marked by horizontal lines. (B) Shown are response magnitudes after stimulation of the contra (C) or ipsilateral (I) eye for the respective groups. Filled circles represent deprived eyes, unfilled circles open eyes. Marked in white on the bars is the statistical comparison between response magnitude after contra- and ipsilateral eye stimulation in the respective group. Contralateral eye $M D$ induces a significant OD-shift in control as well as in PT-animals. But it is prevented in PT-mice after ibuprofen (Ibu) treatment and a 2 week delay between PT-lesion and MD. After ipsilateral eye MD no significant OD-shift was induced in control mice, but in most of the PT-mice groups. 


\section{$\underline{\text { Abstract enriched environment study }}$}

OD-plasticity in mouse visual cortex declines during postnatal development and is absent beyond postnatal day (PD) 110 if mice are raised in standard cages (SC) (Lehmann \& Löwel 2008). Since environmental enrichment (EE) has been shown to promote plasticity in adult rats (Sale et al. 2007; Baroncelli et al. 2010b; Baroncelli et al. 2012; Scali et al. 2012) we raised the question whether enriching the environment of mice would not only prolong or restore the sensitive phase for OD-plasticity but also preserve plasticity after a cortical lesion. We indeed observed that i) EE preserved a juvenile-like OD-plasticity into adulthood, ii) that this effect partially depended on GABAergic inhibition iii) that EE raising did not affect the number of parvalbumin (PV)- positive cells and of perineuronal nets (PNNs) and iiii) that EEhousing can restore already lost plasticity. In addition, iiiii) raising mice in an EE preserved their OD-plasticity even after a PT-stroke.

\subsection{Environmental enrichment extended juvenile OD-plasticity into adulthood and restored it in old mice}

\subsubsection{EE extended juvenile OD-plasticity into adulthood}

OD-plasticity after MD in mouse visual cortex is maximal at 4 weeks of age, declines in 2-3 months old mice and is absent beyond PD 110, if mice are raised in SC (Lehmann \& Löwel 2008). To investigate whether raising mice in an EE could prolong the sensitive phase for OD-plasticity, we transferred pregnant dams to the EE about 7 days before giving birth. Mice were raised in the EE until adulthood ( $\geq$ PD 130). We visualized OD-plasticity by optical imaging of intrinsic signals after 7 days of MD.

In contrast to SC-mice, in mice raised in EE until PD 130, 7 days of MD induced an OD-shift towards the open eye as strong as usually only seen in 4-weeks-old animals (Hofer et al. 2006b; Heimel et al. 2007; Lehmann \& Löwel 2008) (Figure 54 A). Activity in V1 could even become dominated by input from the formerly weaker, ipsilateral eye: the activity patch induced by stimulation of the ipsilateral eye was sometimes even darker than that of the contralateral eye, the ODI became negative, the ODI-histogram was centered left of zero and cold colors prevailed in the two-dimensional OD-map indicating ipsilateral dominance (Figure $53 \mathrm{D})$. The ODI decreased significantly from $0.23 \pm 0.02(\mathrm{n}=4$, PD 130-136) to $0.04 \pm 0.02(\mathrm{n}=7, \quad \mathrm{PD} 119-129 ; \mathrm{P}<0.001$, Bonferroni-adjusted $\mathrm{t}$-test) (Figure $54 \mathrm{~A})$. 
Interestingly, despite the older age this OD-shift was juvenile-like (like in PD 30 mice): the OD-shift was mediated by a decrease of deprived eye responses in V1 (without MD: $1.44 \pm 0.16, \mathrm{n}=4$; with MD: $0.92 \pm 0.07, \mathrm{n}=7 ; \mathrm{P}<0.01$, t-test) (Figure $54 \mathrm{~B}$ ), while, open eye responses remained unchanged, like in PD 30 mice (without MD: $0.91 \pm 0.06 ; n=4$; with MD: $1.02 \pm 0.09, \mathrm{n}=7 ; \mathrm{P}>0.05$, t-test) (Figure $54 \mathrm{~B}$ ). After MD, V1 activation after stimulation of either eye was equally strong (P > 0.05, t-test) (Figure $54 \mathrm{~B}$ ). In adolescent SCmice (PD 80-100) the OD-shift is typically mediated by an increase in the open eye responses (Sawtell et al. 2003; Hofer et al. 2006b; Sato \& Stryker 2008). In contrast to EE-mice, in SCmice OD-plasticity is absent in mice older than PD 110 (Lehmann \& Löwel 2008): After MD, activity patches induced by stimulation of the contralateral (deprived) eye were still darker than those of the ipsilateral (open) eye. The histogram of ODIs was still centered right of zero, the two-dimensional OD-map displayed warm red colors (Figure $53 \mathrm{~B}$ ) and the ODI was not significantly different to SC-mice without MD (Figure $54 \mathrm{~A}$ ).

To test whether EE expanded the sensitive phase for OD-plasticity into even older age, we additionally imaged 6-9 months old mice (mean: PD 220). Again, MD induced a juvenile-like OD-plasticity: the ODI decreased significantly from $0.21 \pm 0.04(\mathrm{n}=5$, PD 204-261) to $-0.11 \pm 0.05(\mathrm{n}=3$, PD 177-196) after MD ( $\mathrm{P}<0.01$, Bonferroni-adjusted t-test) (Figure $54 \mathrm{~A})$. This OD-shift was also mediated by a decrease of deprived eye responses in V1 (contra/ipsi without MD: $1.47 \pm 0.14 / 0.96 \pm 0.12, \mathrm{n}=5$; with $\mathrm{MD}$ : $0.92 \pm 0.10 / 1.06 \pm 0.14$, $\mathrm{n}=3$; for contra values: $\mathrm{P}<0.05$, for ipsi values: $\mathrm{P}>0.05$, t-test) (Figure $54 \mathrm{~B}$ ).

Since ODIs of the two EE-mice groups (PD 130 and PD 220) were not significantly different ( $\mathrm{P}>0.05$, Bonferroni-adjusted t-test), we pooled them for further analysis in the following chapters to the EE $\geq$ PD 130 group (ODI values for this group - without MD: $0.22 \pm 0.02$, $\mathrm{n}=9$, PD 130-261; with MD -0.06 $\pm 0.02, \mathrm{n}=10$, PD 119-196) (Figure 54 A). When looking at the pooled MD-group the pronounced OD-shift became even more obvious (Figure $54 \mathrm{~A}$ ): 8 out of the 10 mice showed even an ipsilateral dominance after MD (including the oldest mouse in this group with PD 196), in one mouse V1 was equally dominated by ipsi- and contralateral eye responses and in another mouse V1 was still slightly dominated by the contralateral eye after MD.

We also examined an additional SC-group with a mean age of PD 235. As in the SC-mice with PD 130 (Lehmann \& Löwel 2008) there was no significant OD-shift: the ODI of mice without MD $(0.24 \pm 0.02, \mathrm{n}=6$, PD 222-249) was not significantly different to mice with MD $(0.29 \pm 0.02, \mathrm{n}=7, \mathrm{PD} 228-248 ; \mathrm{P}>0.05$, Bonferroni-adjusted t-test) (Figure $54 \mathrm{~A})$. After 
MD the ODI of EE-mice ( $\geq$ PD 130) was significantly lower compared to these SC-mice (PD 235) ( $\mathrm{P}<0.001$, Bonferroni-adjusted t-test). The response magnitude after contralateral and ipsilateral eye stimulation in SC-mice did not change after MD (contra: without MD $2.04 \pm 0.22$, with MD $1.78 \pm 0.11$; ipsi: without MD $1.20 \pm 0.19$, with MD $0.98 \pm 0.12$; $\mathrm{P}>0.05$, t-test) and the activity after contralateral eye stimulation remained significantly higher ( $\mathrm{P}<0.001$, t-test) (Figure $54 \mathrm{~B})$. Interestingly, the response magnitude in EE-mice ( $\geq$ PD 130) was lower than in SC-mice (PD 235) in mice without MD. Response magnitude after visual stimulation of the contralateral eye and pooled responses after contra- and ipsilateral eye stimulation were significantly lower in the EE-mice compared to SC-mice (P<0.05, t-test) (Figure $54 \mathrm{~B}$ ). For the age and the sex of the individual mice in the respective groups see Figure 55 and Figure 58.

Taken together, raising mice in an EE extended the sensitive period for OD-plasticity into late adulthood, even in a juvenile-like form. 


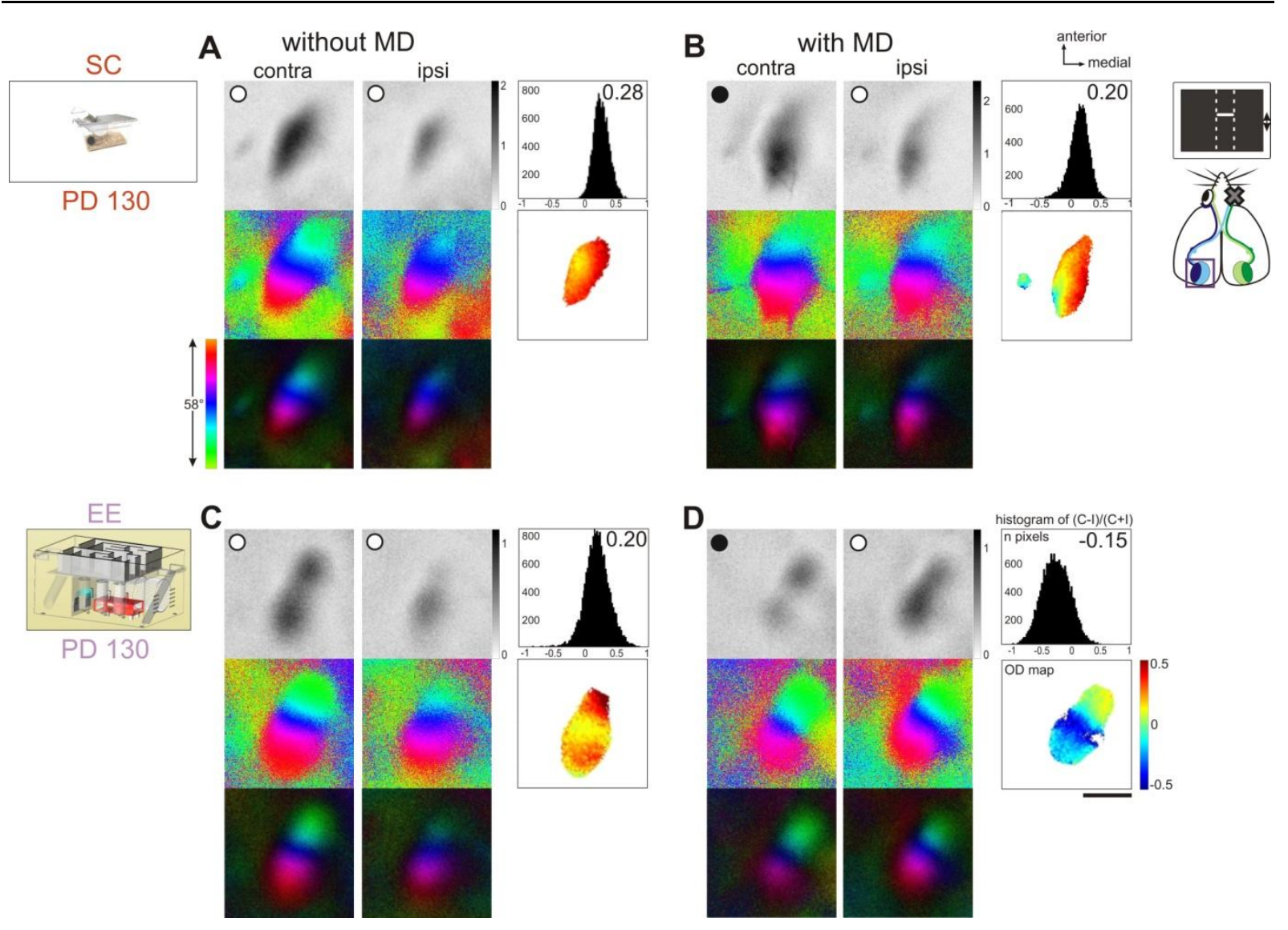

Figure 53: EE from birth prolonged OD-plasticity in PD 130 mice. Maps acquired with optical imaging of intrinsic signals of responses to contra- and ipsilateral eye stimulation in the binocular region of V1 in both SC-mice (A, B) (Lehmann \& Löwel 2008) and in EE-mice (C, D) at PD 130. Maps of mice without MD are shown in the left column (A, C), and maps after MD in the right column (B, D). Gray-scale coded response magnitude maps and their quantification (top), color-coded phase and polar maps of retinotopy (middle, bottom) are illustrated. The magnitude of the optical responses is illustrated as fractional change in reflection $\times 10^{4}$. Black filled circles on the response magnitude maps indicate the deprived eye, unfilled circles non-deprived eyes. In SC- and EE-mice without MD, activity patches evoked by stimulation of the contralateral eye were darker than those after ipsilateral eye stimulation; the average ODI was positive, which is also coded by the warm colored two-dimensional OD-map, indicating contralateral dominance (A, C). A MD of 7 days failed to induce a significant OD-shift in SC-mice (B). In contrast, in EE-mice, MD induced a strong ODshift towards the open eye (D): V1-activity even could become dominated by input from the ipsilateral eye: the response magnitude map of the ipsilateral (non-deprived) eye were darker than those of the deprived contralateral eye, the histogram of OD-scores was shifted to the left, the ODI decreased, and colder colors prevailed in the OD-map. SC maps from Lehmann and Löwel (2008). Scale bar: $1 \mathrm{~mm}$. 


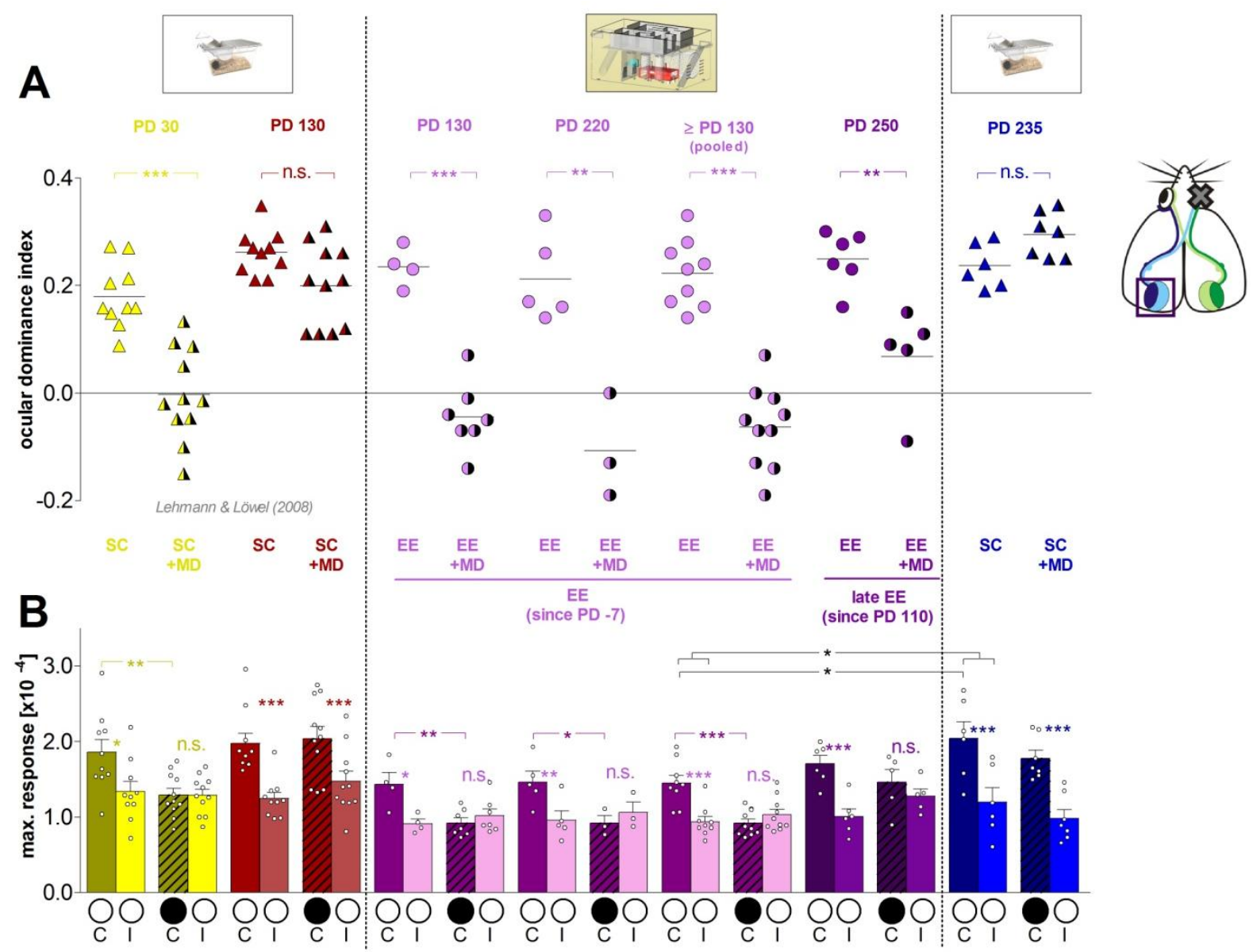

Figure 54: EE prolonged and restored OD-plasticity in adult mice. (A) OD-indices of the left hemisphere. Symbols represent ODI values of individuals; means are marked by horizontal lines. (B) Maximum response magnitude in V1 expressed as fractional change in light reflectance elicited by stimulation of the contralateral (C) or ipsilateral (I) eye in SC- and EE-mice without MD or with MD of the contralateral eye (+MD). Filled circles represent deprived eyes, unfilled circles non-deprived eyes. (A) In SC-mice, OD-plasticity after MD was maximal at PD 30 and was absent beyond PD 110 (Lehmann \& Löwel 2008). Raising mice in an EE not only increased OD-plasticity but created adult animals with juvenile-like OD-plasticity. Seven days of MD in 4-7 months old EE-mice (PD 130 and PD 220) induced a very pronounced OD-shift (A), which was mediated primarily by a reduction of deprived eye responses in V1 (B), like in 30-day-old SC-mice. In contrast, SC-mice over PD 110 (PD 130 and PD 235) did not express OD-plasticity. Moreover, response magnitudes after visual stimulation of the contralateral eye and pooled contra-and ipsilateral eye response were significantly lower in the pooled EE-mice-group ( $\geq$ PD 130) compared to SC-mice. When SC-mice were transferred to EE at PD 110 (group: PD 250), an age in which OD-plasticity was no longer present in SC-mice, OD-plasticity was restored. SC-mice ODI data as indicated from Lehmann \& Löwel (2008). Maximum response values from SC-mice from Lehmann \& Löwel (2008), unpublished. 


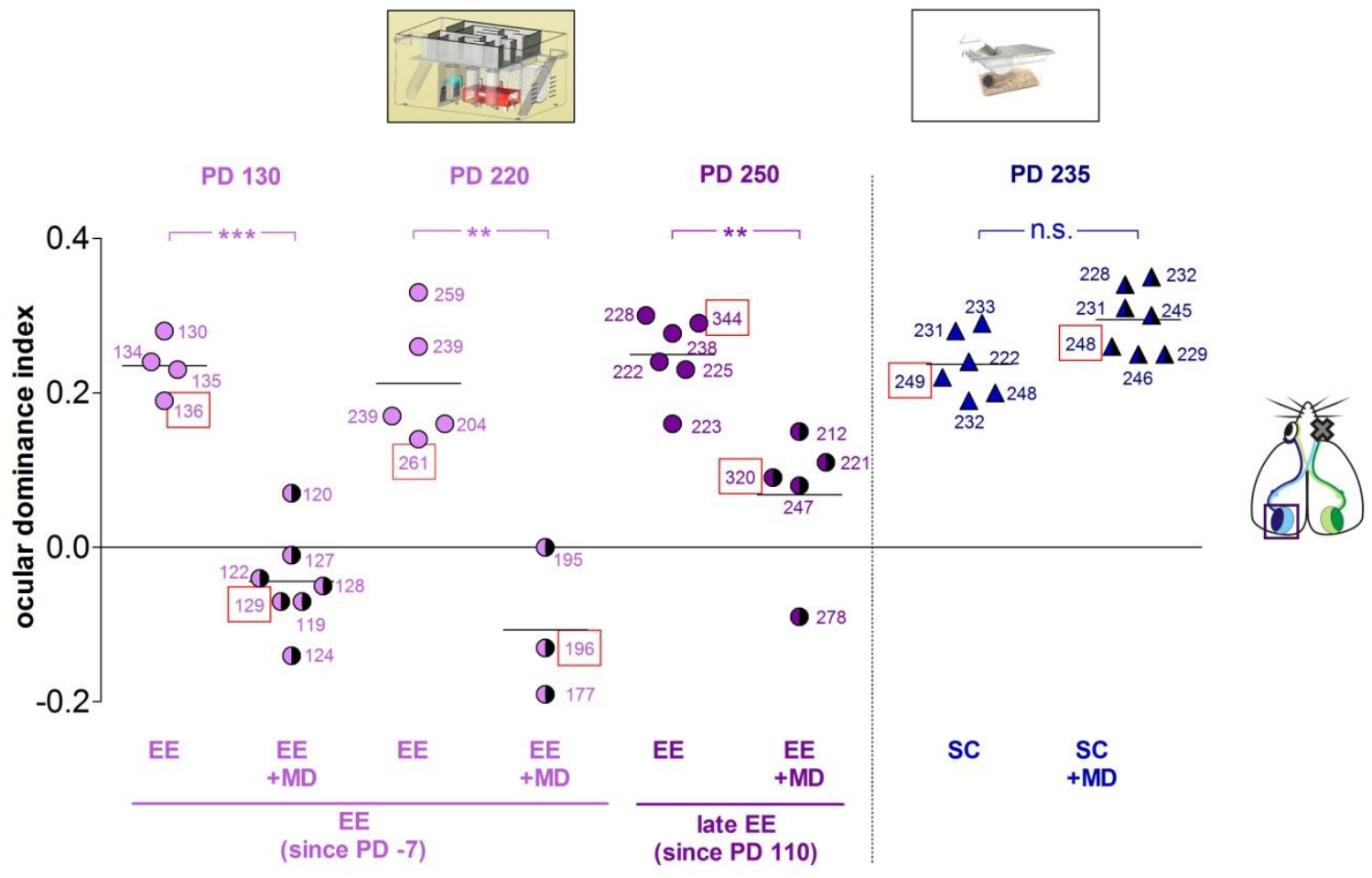

Figure 55: Age (in days) and ODI of the individual EE, late EE and SC animals at the day of optical imaging. The maximum age in the corresponding group is marked with a red outline.

\subsubsection{EE restored OD-plasticity in old mice}

To test whether EE was also able to restore plasticity in SC-mice that were already beyond their sensitive phase for OD-plasticity, we transferred mice from SC into EE at PD 110 (late $\mathrm{EE}$ ) and imaged V1-activites after MD as before. Indeed, MD in late EE-mice caused a significant OD-shift up to an age of 320 days: the response magnitude maps of both, contra(deprived) and ipsilateral (open) eye were almost equally dark, the histogram of the OD-score was shifted to the left and colder blue colors prevailed in the OD-map (Figure 56 D), in contrast to late EE-mice without MD (Figure $56 \mathrm{C}$ ). The average ODI decrease significantly from $0.25 \pm 0.02(\mathrm{n}=6, \mathrm{PD} 222-344)$ to $0.07 \pm 0.04(\mathrm{n}=5, \mathrm{PD} 212-320)$ after $\mathrm{MD}(\mathrm{P}<0.01$, Bonferroni-adjusted t-test) (Figure $54 \mathrm{~A}$ ). In contrast to that in SC-mice of the same age (mean PD 235), MD did not induce a significant OD-shift (Figure $54 \mathrm{~A}$ ): activity patches evoked by stimulation of the contralateral eye were still darker than those after ipsilateral eye stimulation; the average ODI was positive, warm colors prevailed in the OD-map and the ODI was positive, indicating contralateral dominance (Figure $56 \mathrm{~A}, \mathrm{~B}$ ).

The OD-shift in late EE-mice was not clearly mediated by a change in the response strength of either eye: there was neither a significant change in the response of the contralateral (deprived) eye (without MD: $1.71 \pm 0.11$, with $\mathrm{MD}$ : $1.46 \pm 0.17 ; \mathrm{P}>0.05$, t-test) nor of the 
ipsilateral (open) eye (without MD: $1.01, \mathrm{n}=6$; with $\mathrm{MD}: 1.28 \pm 0.10, \mathrm{n}=5, \mathrm{P}>0.05$, t-test)

(Figure $54 \mathrm{~B}$ ). There was no correlation between the time in EE (between 102-210 days) and the ODI ( $\mathrm{p}=0.396, \mathrm{r}=-0.496$, Pearson correlation) (Figure 57). For the age of the individual mice see Figure 55.

To sum up, EE-housing could also restore already lost plasticity: when mice raised in a SC were transferred to EE starting at PD 110 (late EE), an age in which OD-plasticity is no longer present in SC-mice, OD-plasticity could be restored.

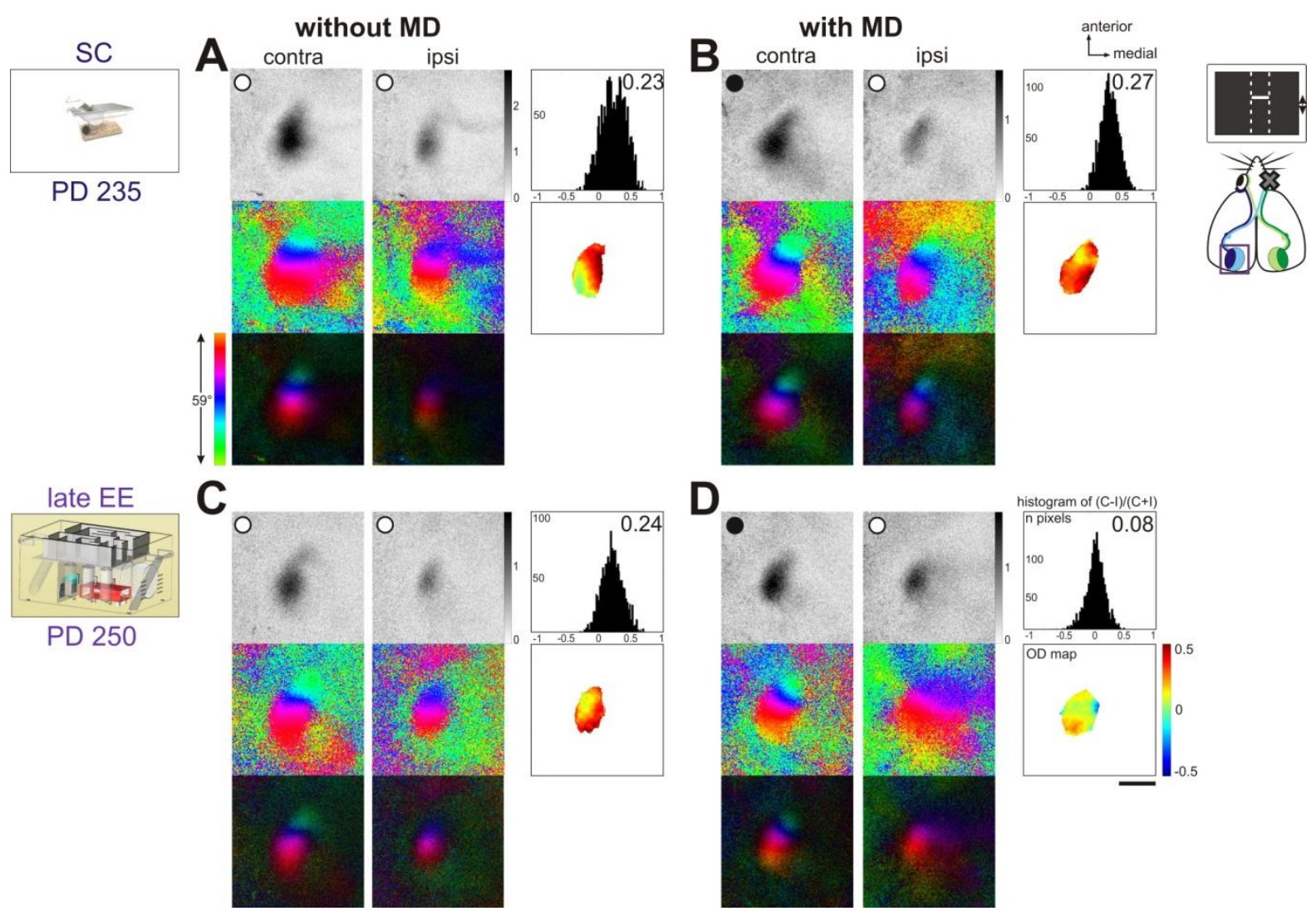

Figure 56: EE restored OD-plasticity in old mice. Maps acquired with optical imaging of intrinsic signals of responses to contra- and ipsilateral eye stimulation in the binocular region of V1 in both SCmice (PD 235) (A, B) and in late EE-mice (PD 250) (C, D). Maps of mice without MD are shown in the left column (A, C), and maps after MD in the right column (B, D). Gray-scale coded response magnitude maps and their quantification (top), color-coded phase and polar maps of retinotopy (middle, bottom) are illustrated. The magnitude of the optical responses is illustrated as fractional change in reflection $\times 10^{4}$. Black filled circles on the response magnitude maps indicate the deprived eye, unfilled circles non-deprived eyes. In SC- and late EE-mice without MD, activity patches evoked by stimulation of the contralateral eye were darker than those after ipsilateral eye stimulation; the average ODI was positive, which is also coded by the warm colored two-dimensional OD-map, indicating contralateral dominance (A, C). A MD of 7 days failed to induce a significant OD-shift in SC-mice (B). In contrast, in late EE-mice, MD induced a significant OD-shift towards the open eye (D): response magnitude after contralateral (deprived) eye stimulation and after ipsilateral (nondeprived) eye stimulation was almost equal, the histogram of OD-scores was shifted to the left, the ODI decreased, and colder colors prevailed in the OD-map. Scale bar: $1 \mathrm{~mm}$. 


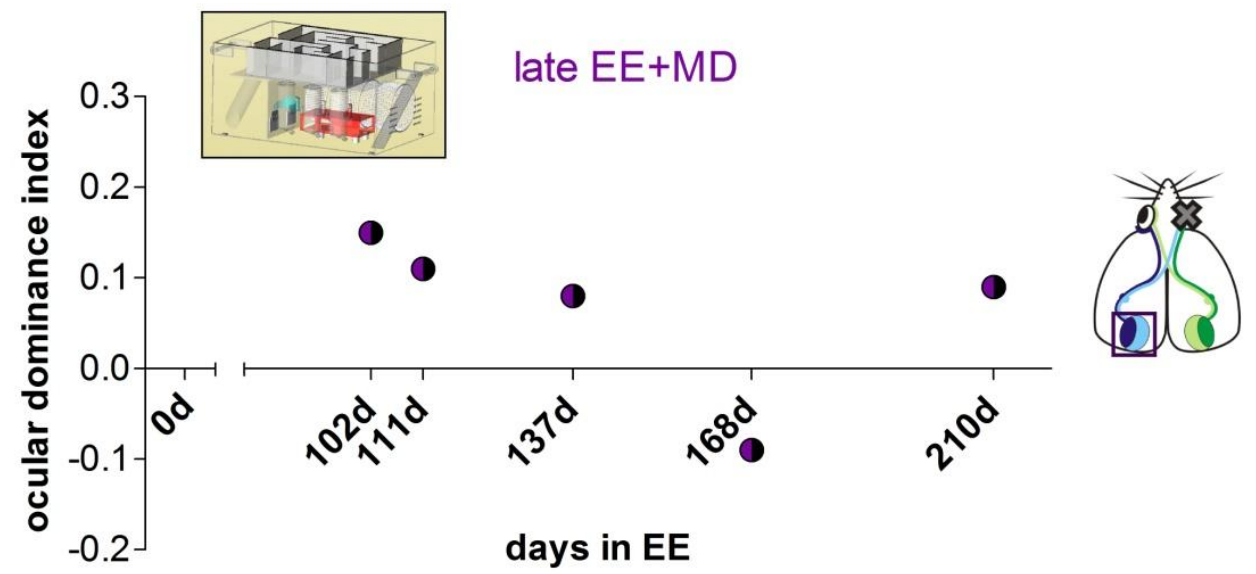

Figure 57: The ODI does not correlate with the time spend in EE in the late EE paradigm. Mice were raised in SC and transferred to the EE with PD 110.

\section{Sex did not influence the effect of EE on OD-plasticity}

For the EE-experiments we used both male as well as female mice. Therefore the question arose whether the sex affected the OD-plasticity in EE-mice.

Usually we exclusively use male mice for our studies in order to avoid potential influence of the changing hormone status in female mice. That is why the SC-mice were all male (Figure 58). For the late EE experiments however, it was inevitable to use solely female mice (Figure 58), because for these experiments litters of several SC-mice were put together in one EEcage at the age of 110 days. Putting together male mice at that age, which are not familiar with each other, would causes serious fights, whereas no such problem exists with female mice. The EE experiments included male and female mice, because we kept all pups born in the EE and separated them later on according to their sex. Most mice with MD in the EE experiments were female (Figure 58). One male mouse in the PD 130 showed the same ODI like the females ones. Nevertheless, to verify that the observed enhanced OD-plasticity in the EE/late EE-mice is not due to a sex difference, we analyzed additional female SC-mice at the age of 143 to 155 days. These experiments confirmed the previous results: female SC-mice older than PD 110 also showed no OD-shift (ODI: $0.25 \pm 0.02, \mathrm{n}=3$; P > 0.05, Bonferroniadjusted t-test) (Figure 58), as previously shown for male mice (Lehmann \& Löwel 2008).

Conclusively, the sex of the mice did not affect OD-plasticity. 


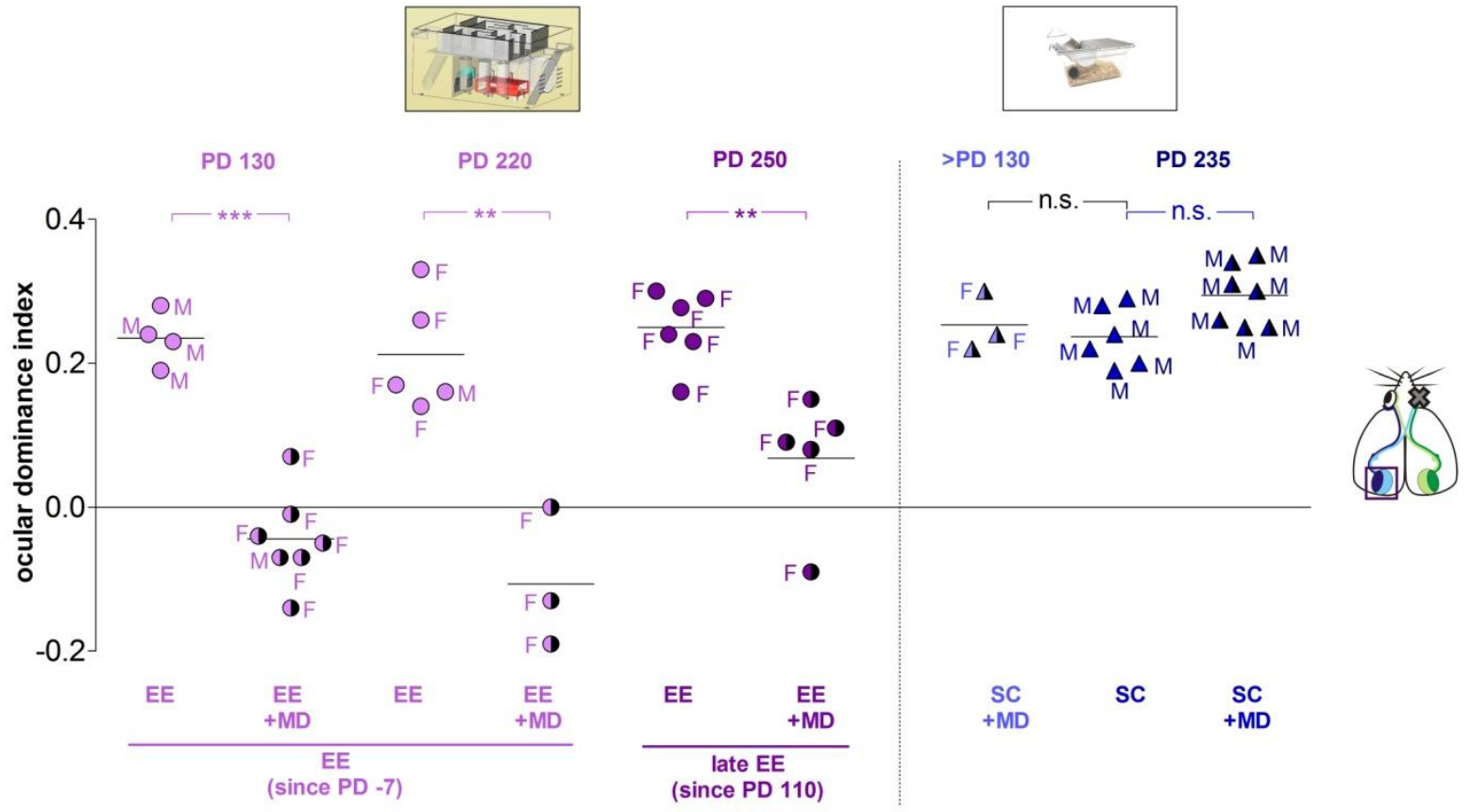

Figure 58: Sex did not influence the effect of EE on OD-plasticity. Shown is the sex of the individual animals assigned to their ODI. Mice in the EE-group (PD 130 and PD 220) were male and female, but most mice with MD were female. The one male mouse in the PD 130 group with MD showed the same ODI like the female mice. Mice in the late EE-group (PD 250) were exclusively female, SC-mice exclusively male. To verify that the observed enhanced OD-plasticity in the EE/late EE-mice is not due to a sex difference, we analyzed additional female > PD 130 SC-mice of. These mice did not show a significant $\mathrm{OD}$-shift after $\mathrm{MD}$. $\mathrm{M}=$ male, $\mathrm{F}=$ female.

\subsubsection{Visual abilities after EE-housing}

In addition of analyzing OD-plasticity with intrinsic signal optical imaging, we investigated visual abilities of the same mice behaviorally in the optomotor system (Prusky et al. 2004). Visual acuity values of the left and right eye before MD were not significantly different and were therefore averaged for further analysis (in all groups $\mathrm{P}>0.05$, t-test).

Without MD. In SC-mice (PD 235) without MD, baseline visual acuity was $0.38 \pm 0.002 \mathrm{cyc} / \mathrm{deg}$ on day 0 and remained stable for 7 days (day 7: $0.38 \pm 0.002 \mathrm{cyc} / \mathrm{deg}$, $\mathrm{n}=7 ; \mathrm{P}>0.05$, Bonferroni-adjusted t-test) (Figure $60 \mathrm{~A}$ ). Raising mice in an EE did not affect visual acuity: visual acuity remained also stable over 7 days (day 0 : $0.38 \pm 0.003 \mathrm{cyc} / \mathrm{deg}$, day 7: $0.38 \pm 0.003 \mathrm{cyc} / \mathrm{deg}, \mathrm{n}=10 ; \mathrm{P}>0.05$, Bonferroni-adjusted ttest) (Figure $60 \mathrm{C})$ and was indistinguishable from SC-mice $\left(\mathrm{F}_{1,15}=0.47, \mathrm{P}>0.05\right.$, ANOVA) (Figure 59). In mice transferred to an EE at an age of 110 days (late EE) visual acuity without MD remained also stable (day 0: $0.38 \pm 0.002 \mathrm{cyc} / \mathrm{deg}$, day $7: 0.38 \pm 0.002 \mathrm{cyc} / \mathrm{deg}, \mathrm{n}=8$ ) 
(Figure $60 \mathrm{E}$ ) and was also neither significantly different to mice raised in $\mathrm{EE}\left(\mathrm{F}_{1,16}=0.00\right.$, $\mathrm{P}>0.05$, ANOVA) nor to SC-mice $\left(\mathrm{F}_{2,17}=0.96, \mathrm{P}>0.05\right.$, ANOVA) (Figure 59).

However, within the EE-mice group without MD, visual acuity of the PD 130 group $(0.394 \pm 0.001, \mathrm{n}=4)$ was significantly different to the PD 220 group $(0.376 \pm 0.001 \mathrm{cyc} / \mathrm{deg}$, $\mathrm{n}=6)$ on day $0(\mathrm{P}<0.001$, Bonferroni-adjusted t-test) (Figure $60 \mathrm{C})$. This difference was most likely due to the origin of mice from different animal facilities. Mice for PD 130 group were obtained from the animal facility of the University of Jena, mice for the PD 220 from the University Medical Center Göttingen. The increase in visual acuity of the open eye after MD was also significantly different between these groups (PD 130: $24.8 \pm 0.47 \%, \mathrm{n}=9$; PD 220: $19.6 \pm 0.52 \%, \mathrm{n}=6)(\mathrm{P}<0.001$, Bonferroni-adjusted t-test) (see also Figure $60 \mathrm{D})$. All mice for the other EE-groups were obtained from the University Medical Center Göttingen. The PD 220 group was also better age matched with the other EE-groups (mean age SC-mice: 235, late EE-mice: 250). Since it also was reported that the age of mice influences the increase of visual acuity after MD (Lehmann \& Löwel 2008), the EE $E_{P D 20}$-group was used for the following comparisons of visual abilities. In the $\mathrm{EE}_{\mathrm{PD} 220}$ group without $\mathrm{MD}$ visual acuity also stayed stable over 7 days (day 0: $0.376 \pm 0.001 \mathrm{cyc} / \mathrm{deg}$, day $7: 0.376 \pm 0.001 \mathrm{cyc} / \mathrm{deg}$, $\mathrm{n}=6$; $\mathrm{P}>0.05$, Bonferroni-adjusted t-test) (Figure $60 \mathrm{C}$ ) and was not significantly different to $\mathrm{SC}$-mice $\left(\mathrm{F}_{1,11}=3.77, \mathrm{P}>0.05\right.$, ANOVA $)$ or late EE-mice $\left(\mathrm{F}_{1,12}=8.06, \mathrm{P}>0.05\right.$, ANOVA) (Figure 59).

With MD. In SC-mice with MD visual acuity of the open eye rose significantly by $21.6 \pm 1.4 \%$ from $0.38 \pm 0.002 \mathrm{cyc} / \mathrm{deg}$ on day 0 to $0.46 \pm 0.005 \mathrm{cyc} / \mathrm{deg}$ on day $7(\mathrm{n}=8$; $\mathrm{P}<0.001$, Bonferroni-adjusted t-test) (Figure 59, Figure $60 \mathrm{~B}$ ). In $\mathrm{EE}_{\mathrm{PD} 220}$-mice the visual acuity was also significantly increased after MD by $19.6 \pm 0.52 \%$ (day 0: $0.38 \pm 0.003 \mathrm{cyc} / \mathrm{deg}$, day 7: $0.46 \pm 0.004 \mathrm{cyc} / \mathrm{deg}, \mathrm{n}=6 ; \mathrm{P}<0.001$, Bonferroni-adjusted ttest) (Figure $60 \mathrm{D}$ ) (pooled $\mathrm{EE}_{\mathrm{PD} 130 \& \mathrm{PD} 220:} 22.7 \pm 0.8 \%$, day 0: $0.39 \pm 0.002 \mathrm{cyc} / \mathrm{deg}$, day 7: $0.48 \pm 0.005 \mathrm{cyc} / \mathrm{deg}, \mathrm{n}=15 ; \mathrm{P}<0.001$, Bonferroni-adjusted t-test). This increase of $\mathrm{EE}_{\mathrm{PD} 220^{-}}$ mice was not significantly different to $\mathrm{SC}$-mice $\left(\mathrm{F}_{1,12}=0.58, \mathrm{P}>0.05\right.$, ANOVA) (Figure 59). In late EE-mice, the visual acuity was also significantly enhanced by $19.4 \pm 2.7 \%$ from $0.39 \pm 0.002 \mathrm{cyc} / \mathrm{deg}$ on day 0 to $0.46 \pm 0.01 \mathrm{cyc} / \mathrm{deg}$ after 7 days of $\mathrm{MD}(\mathrm{n}=6 ; \mathrm{P}<0.05$, Bonferroni-adjusted t-test) (Figure $60 \mathrm{~F}$ ). This enhancement was neither significantly different to mice raised in $\mathrm{EE}_{\mathrm{PD} 220}\left(\mathrm{~F}_{1,10}=1.59, \mathrm{P}>0.05\right.$, ANOVA $)$ nor to $\mathrm{SC}$-mice $\left(\mathrm{F}_{1,12}=\right.$ 0.96, P > 0.05, ANOVA) (Figure 59). 
Taken together, neither the visual acuity without MD nor the visual acuity increase of the open eye after MD was affected by EE-housing.

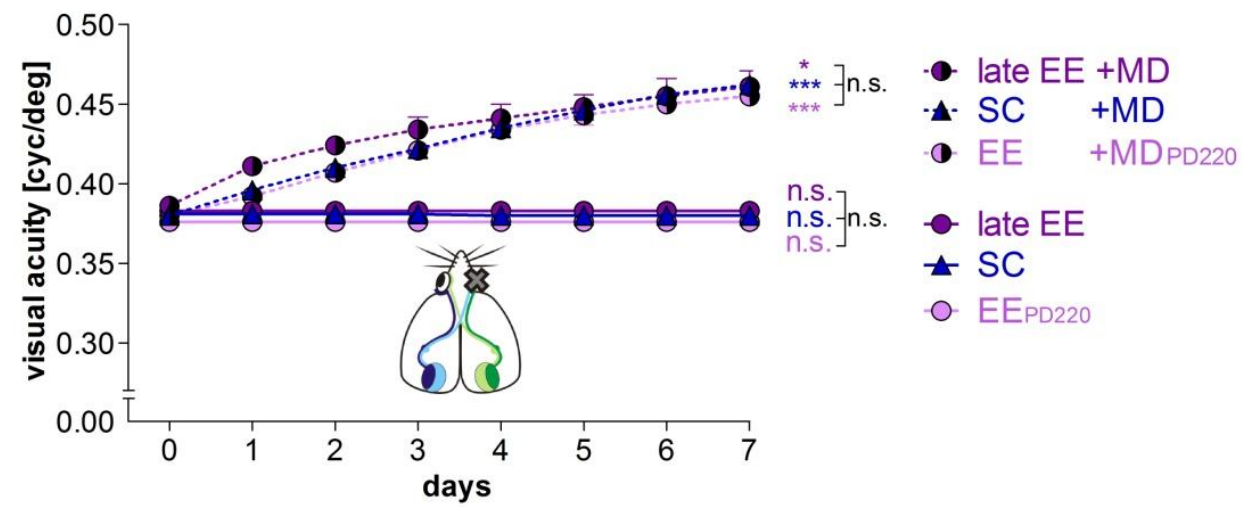

Figure 59: Visual acuity and visual acuity enhancement after MD was not affected by EEhousing. In all groups without $\mathrm{MD}$ visual acuity remained stable over 7 days and all groups $\left(\mathrm{EE}_{\mathrm{PD} 220}\right.$, late EE, SC) were indistinguishable from each other. After MD, visual acuity of the open eye increased significantly in all groups. The increase over the 7 days of MD was the same in all groups. 


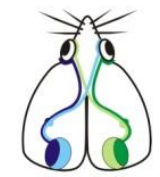

A

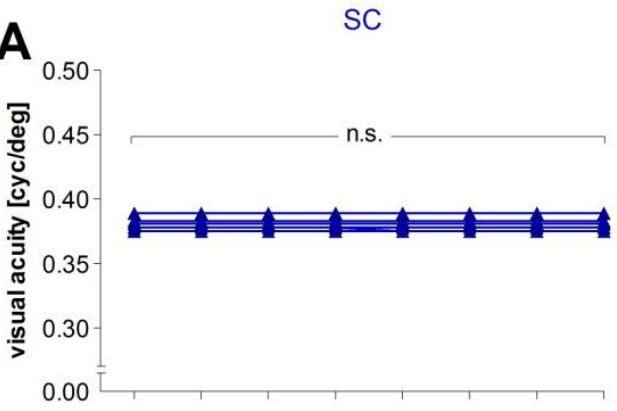

EE
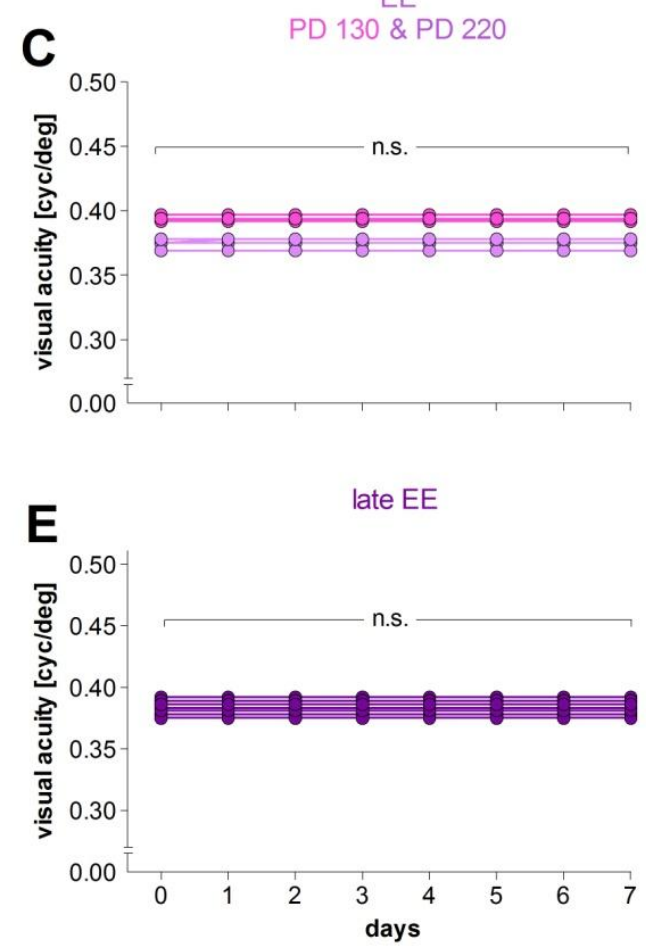

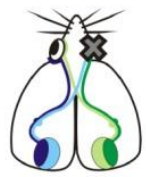

B

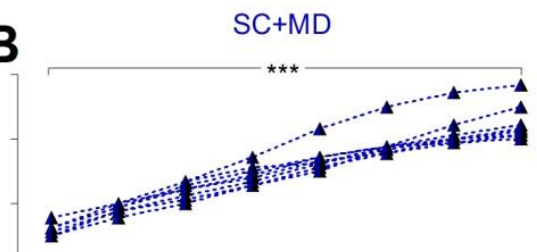

$\mathrm{EE}+\mathrm{MD}$

D PD 130 \& PD 220

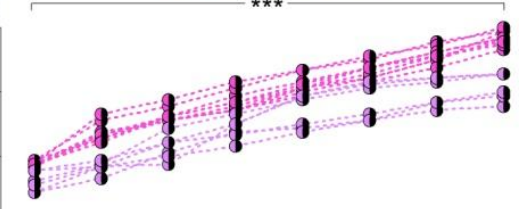

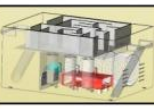
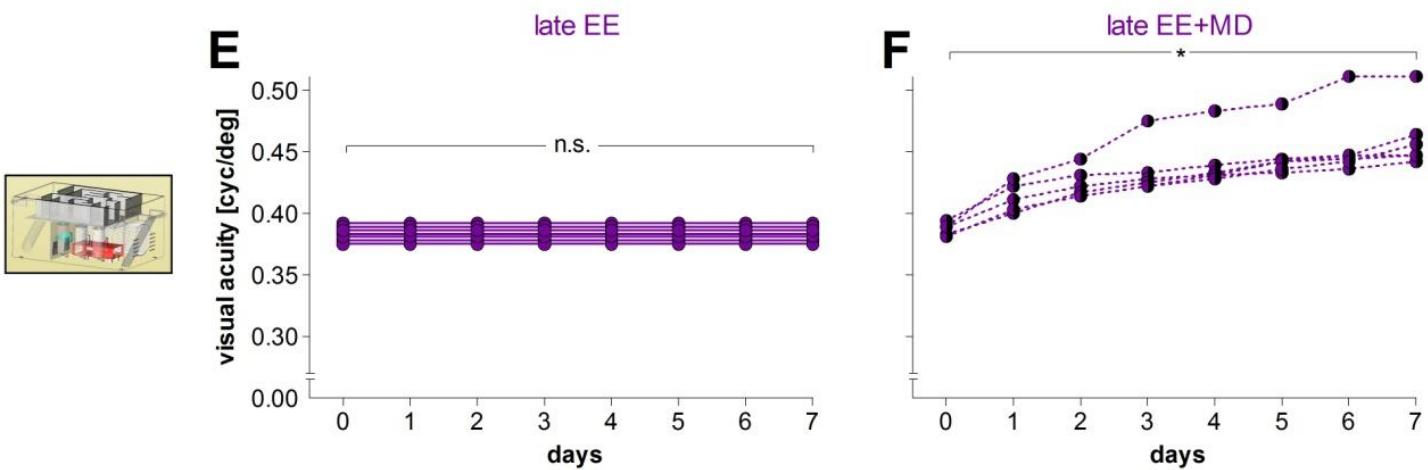

Figure 60: Individual values of visual acuity of mice raised in (A, B) SC, (C, D) EE or (E, F) that were transferred to $\mathrm{EE}$ at $\mathrm{PD} 110$ (late $\mathrm{EE}$ ) without $\mathrm{MD}$ (left column) or with $\mathrm{MD}$ of the right eye (right column). Each line represents one animal. In all groups without MD (A, C, E) visual acuity remained stable over 7 days. After MD visual acuity of the open eye increased significantly in all groups (B, D, F).

Contrast sensitivity between the groups without MD was not significantly different (at all frequencies: P > 0.05, Bonferroni-adjusted t-test) (Figure 61). Contrast sensitivity values at a spatial frequency of $0.064 \mathrm{cyc} / \mathrm{deg}$, day $0: \mathrm{SC}: 15.5 \pm 0.30(\bumpeq 6 \%$ contrast, $\mathrm{n}=7), \mathrm{EE} \mathrm{PD}_{130}$ $14.2 \pm 0.35(\bumpeq 7 \%$ contrast, $\mathrm{n}=4), \mathrm{EE} \mathrm{PD}_{220} 15.2 \pm 0.53(\bumpeq 7 \%$ contrast, $\mathrm{n}=6)$, late $\mathrm{EE}$ $15.4 \pm 0.28(\hat{=} 7 \%$ contrast, $\mathrm{n}=8)$. Without $\mathrm{MD}$ contrast sensitivity remained stable in all 
groups (for each group at all frequencies: $\mathrm{P}>0.05$, Bonferroni-adjusted t-test) (Figure $62 \mathrm{~A}, \mathrm{C}, \mathrm{E}, \mathrm{G})$. After MD contrast sensitivity of the open eye increased significantly in all groups ( $\mathrm{n}$ in the groups: $\mathrm{SC}=8, \mathrm{EE}_{\mathrm{PD} 130}=9, \mathrm{EE}_{\mathrm{PD}_{220}}=6$, late $\mathrm{EE}=6$; in all groups $\mathrm{P}<0.01-0.001$, Bonferroni-adjusted t-test) (Figure $62 \mathrm{~B}, \mathrm{D}, \mathrm{F}, \mathrm{H}$; Table 7), so that the contrast sensitivity at day 7 was significantly higher in mice with MD than in mice without MD (in all groups $\mathrm{P}<0.01-0.001$, Bonferroni-adjusted t-test) (Figure 61). After 7 days of MD contrast sensitivities were not significantly different between the groups except for some slight differences between SC- and $\mathrm{EE}_{\mathrm{PD} 220}$-mice (see Figure 61 for statistical comparison).

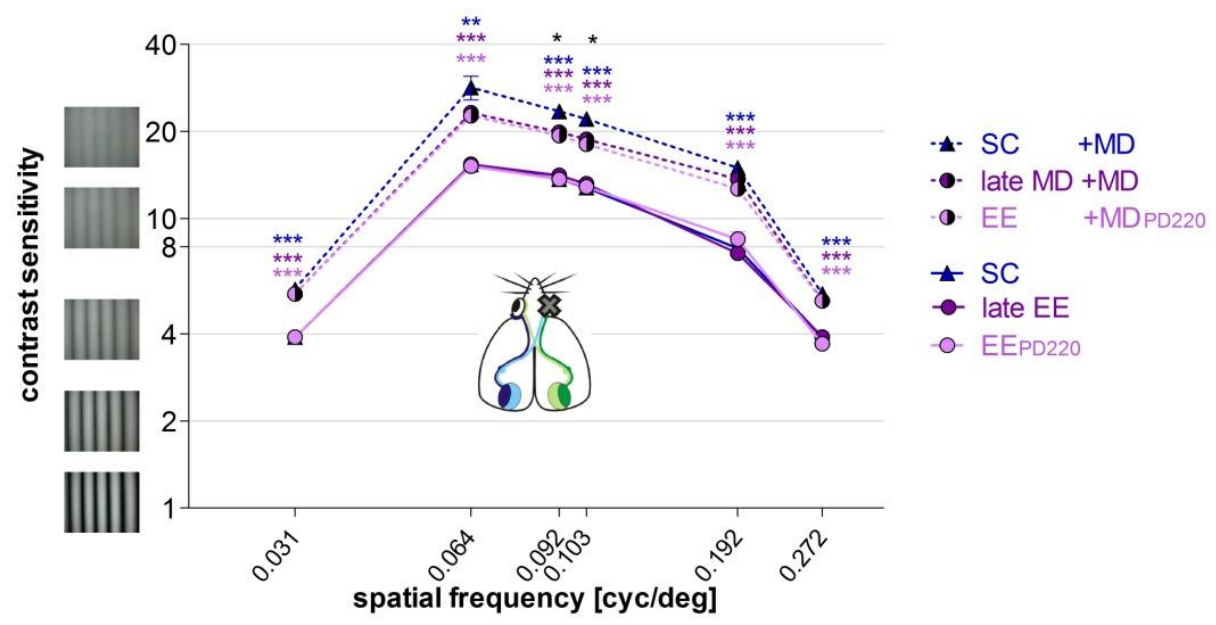

Figure 61: Contrast sensitivity of SC-, $\mathbf{E E}_{\mathbf{P D 2 2 0}}$ and late EE-mice. Shown are the contrast sensitivities on day 7 at all measured frequencies. Groups without MD were not significantly different irrespective of SC- or EE-housing. In all groups contrast sensitivity of the open eye increased significantly after MD: contrast sensitivity at day 7 was significantly higher in mice with MD than in mice without MD (significance for the respective group indicated in the according color). After MD contrast sensitivities were not significantly different between the groups except for some slight differences between $\mathrm{SC}$ - and $\mathrm{EE}_{\mathrm{PD} 220}$-mice (the black asterisk $\left(^{*}\right)$ symbol indicates a significant difference). 


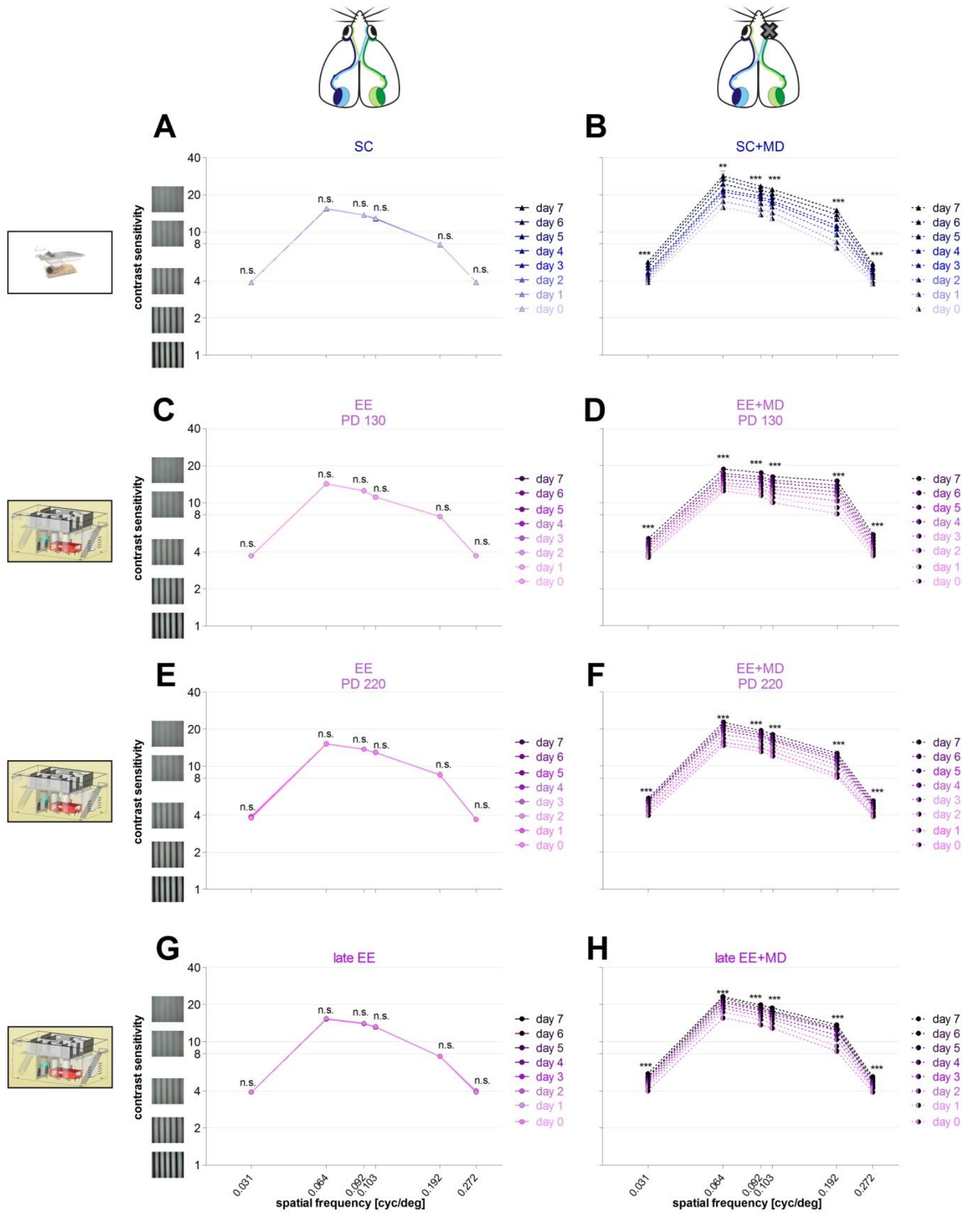

Figure 62: Mean contrast sensitivities for the individual days and spatial frequencies of mice raised in SC (A, B), EE until PD 130 (C, D) or until PD $220(\mathrm{E}, \mathrm{F})$ or that were transferred to EE at PD 110 (late EE) (G, H) without MD (left column) or with MD of the right eye (right column). In all groups without MD visual acuity remained stable over 7 days (A, C, E, G). After MD contrast sensitivity of the open eye increased significantly in all groups after MD (B, D, F, H). 
Table 7: Contrast sensitivity of the open eye increased significantly after MD in mice raised in SC and EE and late EE. Average contrast values \pm S.E.M. on day 0 and day 7 after MD and statistical significance for the increase over these days (Bonferroni-adjusted t-test).

\begin{tabular}{|c|c|c|c|}
\hline Frequencies [cyc/deg] & Contrast sensitivity day 0 & Contrast sensitivity day 7 & $\mathrm{P}$ \\
\hline $\begin{array}{r}\text { SC+MD } \\
0.031 \\
0.064 \\
0.092 \\
0.103 \\
0.192 \\
0.272\end{array}$ & $\begin{array}{c}3.9 \pm 0.03 \\
15.8 \pm 0.36 \\
13.8 \pm 0.27 \\
12.9 \pm 0.23 \\
7.4 \pm 0.14 \\
3.8 \pm 0.02\end{array}$ & $\begin{array}{c}5.7 \pm 0.12 \\
28.4 \pm 2.68 \\
23.5 \pm 1.02 \\
22.1 \pm 0.93 \\
15.0 \pm 0.67 \\
5.5 \pm 0.14\end{array}$ & $\begin{array}{l}<0.001 \\
<0.001 \\
<0.001 \\
<0.001 \\
<0.001 \\
<0.001\end{array}$ \\
\hline $\begin{array}{c}\text { EE+MD (PD 130) } \\
0.031 \\
0.064 \\
0.092 \\
0.103 \\
0.192 \\
0.272\end{array}$ & $\begin{array}{c}3.6 \pm 0.03 \\
12.4 \pm 0.16 \\
11.4 \pm 0.18 \\
10.0 \pm 0.15 \\
8.1 \pm 0.10 \\
3.7 \pm 0.02\end{array}$ & $\begin{array}{c}5.1 \pm 0.09 \\
18.7 \pm 0.42 \\
17.5 \pm 0.40 \\
16.2 \pm 0.43 \\
15.0 \pm 0.47 \\
5.5 \pm 0.15\end{array}$ & $\begin{array}{l}<0.001 \\
<0.001 \\
<0.001 \\
<0.001 \\
<0.001 \\
<0.001\end{array}$ \\
\hline $\begin{array}{c}\text { EE+MD (PD 220) } \\
0.031 \\
0.064 \\
0.092 \\
0.103 \\
0.192 \\
0.272\end{array}$ & $\begin{array}{c}4.0 \pm 0.12 \\
14.7 \pm 0.44 \\
13.1 \pm 0.44 \\
12.1 \pm 0.44 \\
8.1 \pm 0.33 \\
3.9 \pm 0.11\end{array}$ & $\begin{array}{c}5.5 \pm 0.23 \\
22.7 \pm 1.06 \\
19.4 \pm 0.56 \\
18.1 \pm 0.57 \\
12.7 \pm 0.62 \\
5.2 \pm 0.25\end{array}$ & $\begin{array}{l}<0.001 \\
<0.001 \\
<0.001 \\
<0.001 \\
<0.001 \\
<0.001\end{array}$ \\
\hline $\begin{array}{r}\text { Late EE+MD } \\
0.031 \\
0.064 \\
0.092 \\
0.103 \\
0.192 \\
0.272\end{array}$ & $\begin{array}{c}4.0 \pm 0.13 \\
15.6 \pm 0.33 \\
13.7 \pm 0.17 \\
12.9 \pm 0.14 \\
8.4 \pm 0.24 \\
3.9 \pm 0.10\end{array}$ & $\begin{array}{c}5.5 \pm 0.17 \\
23.2 \pm 0.95 \\
19.9 \pm 0.47 \\
18.8 \pm 0.57 \\
13.7 \pm 0.61 \\
5.2 \pm 0.18\end{array}$ & $\begin{array}{l}<0.001 \\
<0.001 \\
<0.001 \\
<0.001 \\
<0.001 \\
<0.001\end{array}$ \\
\hline
\end{tabular}

\subsubsection{Diazepam treatment only partly abolished OD-plasticity}

Since it was hypothesized that the EE-effect on OD-plasticity in rats is mediated via a reduction of GABAergic ( $\gamma$-aminobutyric acid) inhibition (Sale et al. 2007; Baroncelli et al. 2010b; Baroncelli et al. 2012), we next tested whether a reduction of GABAergic inhibition in the visual cortex was crucial for the prolongation of OD-plasticity in our EE-mice. To this end, we applied diazepam (1mg/kg, daily intraperitoneal injections) to a separate group of EEmice older than PD 130 for the 7 day MD-period. Diazepam is a positive allosteric modulator of the $\mathrm{GABA}_{\mathrm{A}}$ receptor that has been commonly used to increase GABA receptor mediated inhibition in the visual cortex (Hensch et al. 1998; Fagiolini et al. 2004). The relatively high dosage for intraperitoneal diazepam treatment of $30 \mathrm{mg} / \mathrm{kg}$, which was indicated in the literature (Huopaniemi et al. 2004; Kanold et al. 2009) caused death or pronounced lethargy after application in wild-type mice. Hence we tested several dosages, where a dose of $1 \mathrm{mg} / \mathrm{kg}$ 
proved to be low enough, that the mice maintained their normal behavior (personal communication Bianka Götze).

First of all, we checked whether our diazepam treatment protocol and dosage were in fact capable of preventing OD-plasticity. Therefore, we performed experiments with adult SCanimals around PD 90, an age where OD-plasticity can typically be induced with 7 days of MD (Figure 64 A) (Lehmann \& Löwel 2008). When SC-mice were treated with diazepam during the MD-period, the OD-shift was totally abolished and contralateral dominance persisted. V1 was still stronger activated after contralateral (deprived) eye stimulation $(1.81 \pm 0.22, \mathrm{n}=5)$ than after ipsilateral (open) eye stimulation $(1.12 \pm 0.16, \mathrm{n}=5)$ in the imaging experiments $(\mathrm{P}<0.01$, t-test) (Figure $64 \mathrm{C})$. The same was visible in the response magnitude maps (Figure $63 \mathrm{~A}$ ): the response magnitude maps of the contralateral eye were still darker than those of the ipsilateral eye, the OD-map displayed warm colors and the ODI was positive. The ODI-values $(0.25 \pm 0.02, \mathrm{n}=5$, PD 81-91) were not significantly different from PD 90 mice without MD (P > 0.05, Bonferroni-adjusted t-test) and significantly higher than in untreated PD 90 mice with MD (P < 0.001, Bonferroni-adjusted t-test) (Figure $64 \mathrm{~A})$. Consequently, the protocol we used to increase GABAergic inhibition by diazepam treatment during MD was capable of preventing OD-plasticity in PD 90 SC-mice.

In EE-mice diazepam treatment reduced, but did not completely abolish the OD-shift after MD. V1-activity in the response magnitudes maps after contra- and ipsilateral eye stimulation was more similar, the OD-histogram was slightly shifted to the left, the colors in the OD-map were colder and the ODI reduced (Figure $63 \mathrm{~B}$ ). The effect of diazepam on OD-plasticity of individual EE-mice was quite diverse. ODI values ranged from -0.05 , corresponding to a strong OD-plasticity, up to 0.26 , corresponding to no OD-plasticity (Figure $64 \mathrm{~B}$ ). On average, V1 was still partly contralateral dominated (mean ODI: $0.11 \pm 0.03$; maximum response contralateral eye: $1.22 \pm 0.06$, ipsilateral eye: $0.97 \pm 0.04, \mathrm{P}<0.01$, t-test; $\mathrm{n}=9$, PD 162-190) (Figure 64 B, D). Compared to EE-mice without MD (pooled group of the data described before (3.4.1); ODI: $0.22 \pm 0.02, \mathrm{n}=9$, PD 130-261) the OD-shift was still significant $(\mathrm{P}<0.05$, Bonferroni-adjusted t-test) (Figure $64 \mathrm{~B})$. However, the shift induced was significantly smaller compared to EE-mice with MD (ODI: $-0.06 \pm 0.02, \mathrm{n}=10$, PD 119196) $(\mathrm{P}<0.01$, Bonferroni-adjusted t-test) (Figure $64 \mathrm{~B})$.

Consequently, diazepam administration just partly prevented the EE-induced prolongation of OD-plasticity. 


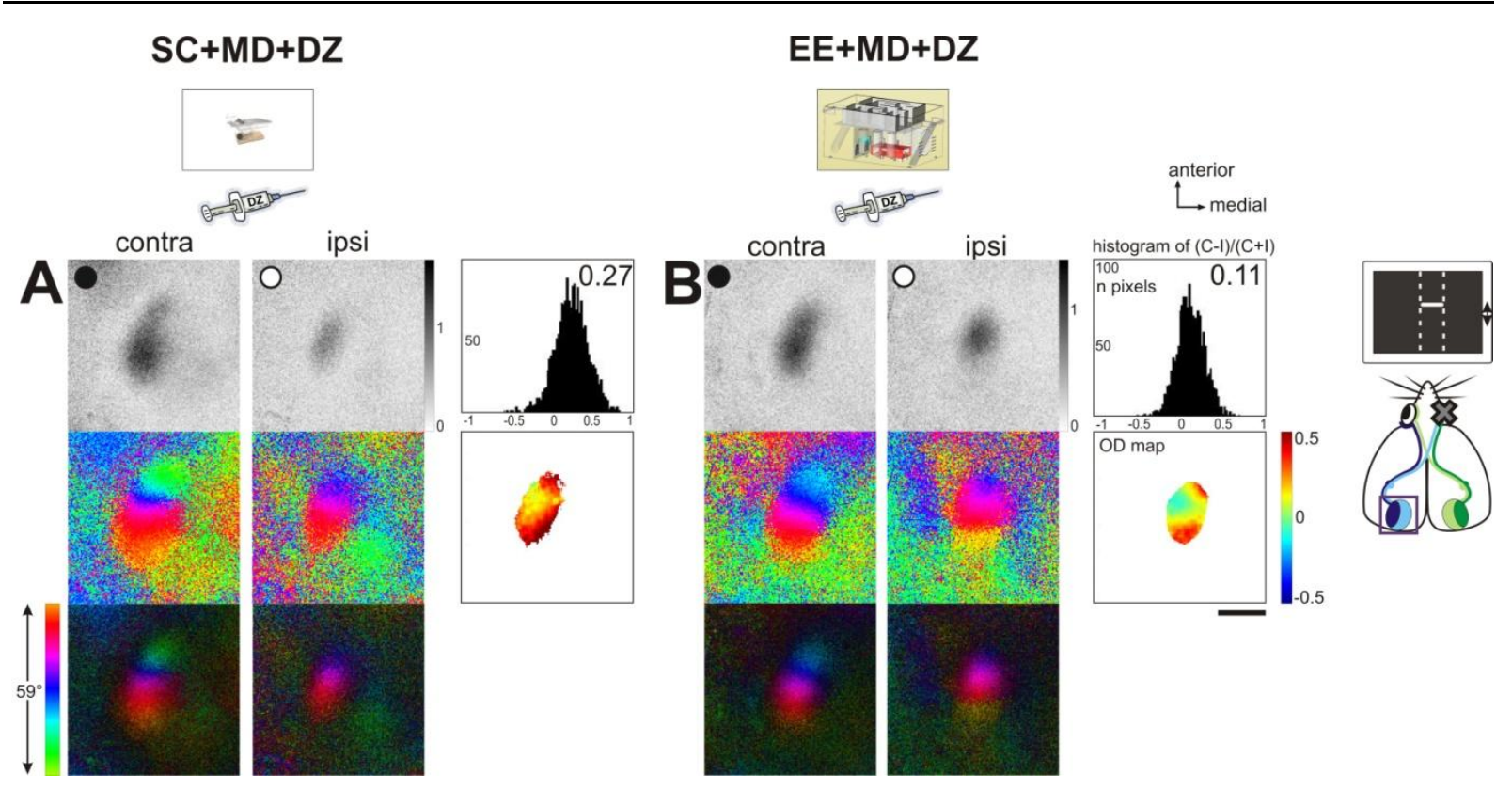

Figure 63: Diazepam treatment to increase GABAergic inhibition during MD reduced but did not completely abolish OD-plasticity in EE-mice. Optically imaged maps of V1 responses after contra- and ipsilateral (ipsi) eye stimulation in SC- (A) and EE-mice after MD and diazepam (DZ) treatment. Both gray-scale coded response magnitude maps and their quantification (top), and colorcoded phase and polar maps of retinotopy (middle, bottom) are illustrated. The magnitude of the optical responses is illustrated as fractional change in reflection $\times 10^{4}$. Black filled circles on the response magnitude maps indicate the deprived eye, unfilled circles non-deprived eyes. For each experiment, the histogram of OD-scores, the average OD-index (ODI), and the corresponding twodimensional OD-maps are included (ODI values are color-coded: blue represents negative, red positive values). (A) In SC-animals around PD 90, an age where OD-plasticity can typically still be induced in SC-mice, diazepam treatment totally abolished an OD-shift: the response magnitude maps of the contralateral (deprived) eye were still darker than those of the ipsilateral (open) eye, the ODI was still positive and warm colors still prevailed in the OD-map. Thus, contralateral dominance persisted. (B) In mice raised in EE (> PD 130) and treated with diazepam to increase GABAergic inhibition, a slight OD-shift could be observed: the darkness of response magnitude maps of the deprived eye and open eye were more similar, the ODI was reduced and cold colors were also present in the OD-map. Scale bar: $1 \mathrm{~mm}$. 


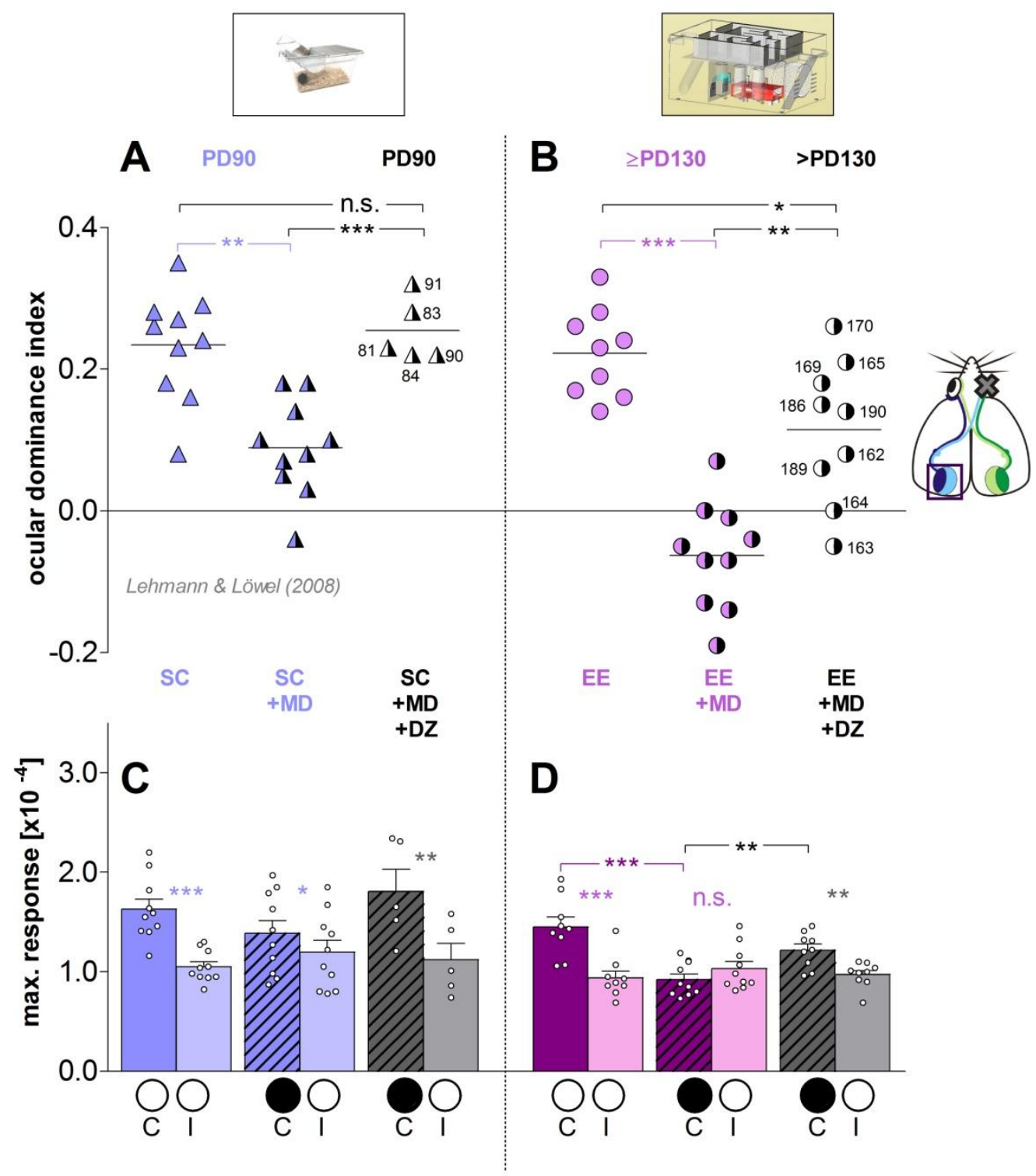

Figure 64: Increased GABAergic inhibition only partly abolished OD-plasticity in EE-mice. (A, B) Shown are the ODIs, symbols represent single animals, horizontal bars the mean value of the group. Numbers at the symbols indicate the age of the individual animal (in days) at the day of optical imaging. (C, D) Displayed are the maximum responses in the binocular zone of V1 after stimulation of the contralateral (C) and ipsilateral (I) eye, respectively. Black filled circles represent deprived eyes, unfilled circles non-deprived eyes. Only significant changes in response strength are labeled. In PD 90 SC-mice MD induced a significant OD-shift toward the open eye (A). The difference in the response strength in V1 after contralateral and ipsilateral eye stimulation was lower (C). Treatment with diazepam (DZ) during MD to increase inhibition, completely abolished this OD-shift (A), contralateral dominance persisted, which can also be seen in the response strength (C). In mice raised in EE ( $\geq$ PD 130) MD induced a significant reduction of ODIs (B), mediated by a decrease in closed eye responses (D). However, if mice that were treated with diazepam this OD-shift after MD was partly abolished (B) and V1 activation was still stronger after contralateral than after ipsilateral eye stimulation (D). Data of PD 90 SC- and SC+MD-mice from Lehmann and Löwel (2008). 


\subsubsection{Diazepam treatment did not affect enhancement of visual acuity}

Visual acuity of the open eye after MD of PD 90 SC-mice treated with diazepam was increased significantly from $0.38 \pm 0.001 \mathrm{cyc} / \mathrm{deg}$ before $\mathrm{MD}$, to $0.45 \pm 0.005 \mathrm{cyc} / \mathrm{deg}$ after MD (= $19.2 \pm 1.6 \%, \mathrm{n}=6 ; \mathrm{P}<0.01$, Bonferroni-adjusted t-test) (Figure 66 A) and was not significantly different to untreated SC-mice with $\mathrm{MD}\left(\mathrm{F}_{1,12}=2.68, \mathrm{P}>0.05\right.$, ANOVA $)$ (Figure 65). Mice raised in EE and treated with diazepam during the MD-period also showed a significant increase in the visual acuity of the open eye from $0.38 \pm 0.002 \mathrm{cyc} / \mathrm{deg}$ on day 0 up to $0.45 \pm 0.004 \mathrm{cyc} / \mathrm{deg}$ on day 7 after $\mathrm{MD}(=17.7 \pm 0.9 \%, \mathrm{n}=9 ; \mathrm{P}<0.001$, Bonferroniadjusted t-test) (Figure 65, Figure 66 B). This increase was neither significantly different to $\mathrm{EE}_{\mathrm{PD} 220}$-mice with $\mathrm{MD}\left(\mathrm{F}_{1,13}=0.30, \mathrm{P}<0.05\right.$, ANOVA $)$ nor to SC-mice with diazepam treatment and $\mathrm{MD}\left(\mathrm{F}_{1,13}=1.48, \mathrm{P}>0.05\right.$, ANOVA) (Figure 65). The $\mathrm{PD}_{220} \mathrm{EE}$ group (with an age range of PD 177-266) was chosen as a comparison group, because it was better agematched to the EE-group with MD and diazepam treatment (PD 162-190), than the PD 130 EE group with MD (PD 119-130) and because mice were obtained from the same animal facility.

Concluding, diazepam treatment did not affect the visual acuity increase of the open eye after $\mathrm{MD}$ in SC- or EE-mice.

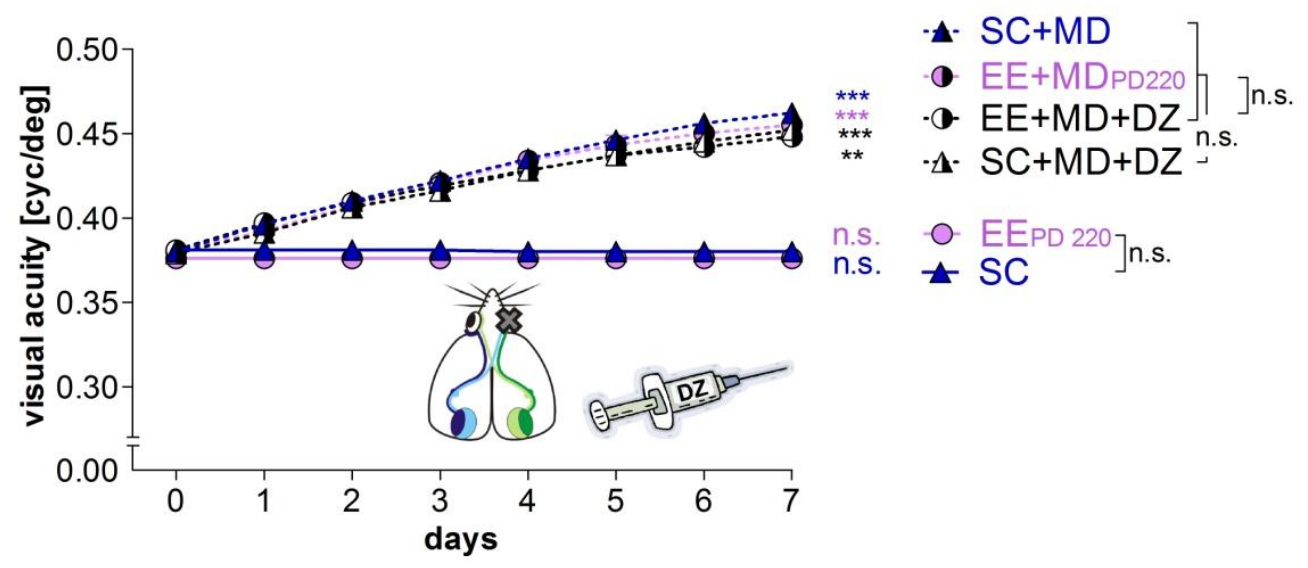

Figure 65: Enhancement of vision after MD was not affected by diazepam treatment. In the groups without MD visual acuity remained stable over 7 days and was indistinguishable between $\mathrm{EE}_{\mathrm{PD} 220}$ and SC-mice. After MD visual acuity of the open eye increased significantly in all groups. The increase was not significantly different between EE-mice with and without diazepam (DZ) treatment. And the increase was also not significantly different between SC-mice with and without diazepam treatment and SC-mice with treatment and EE-mice with treatment. 


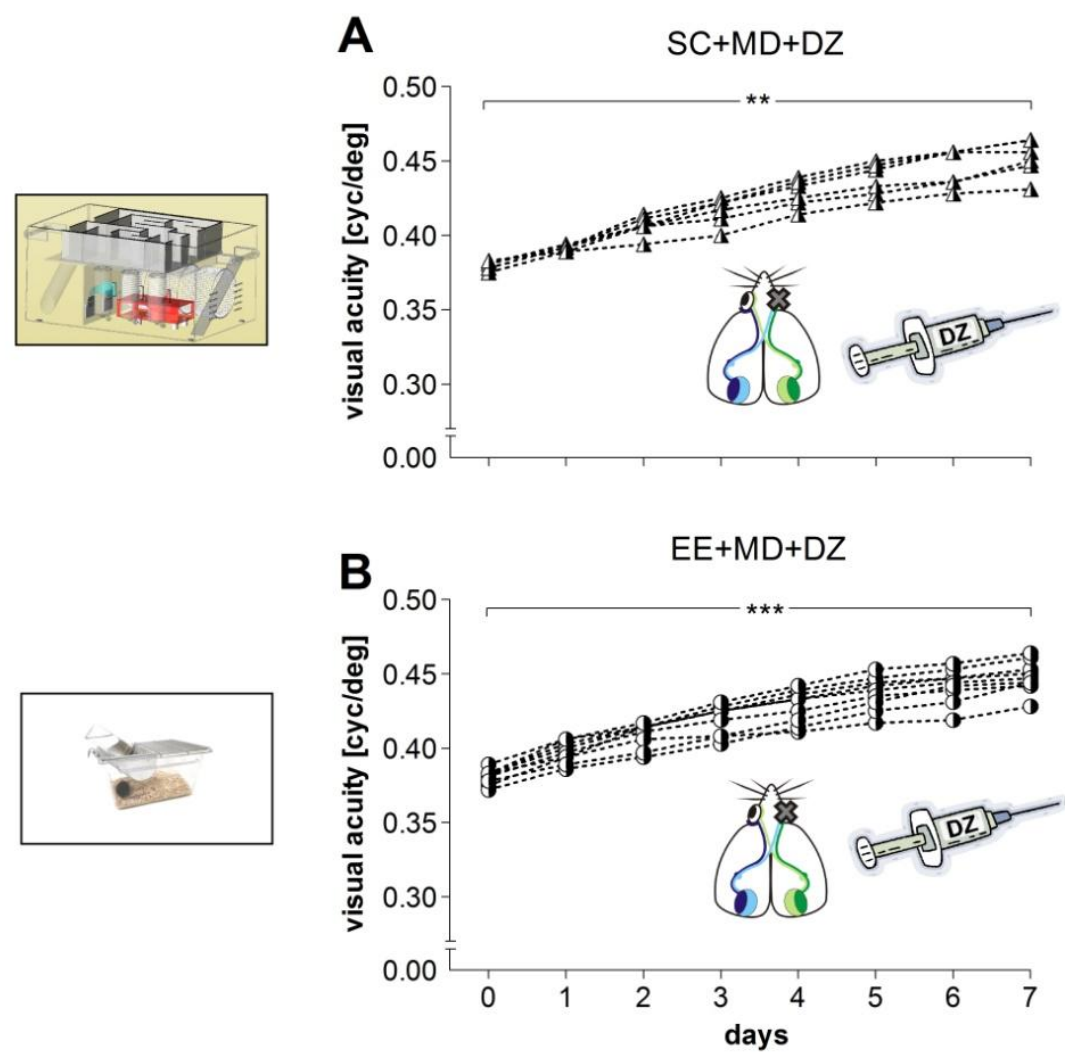

Figure 66: Individual values of visual acuity enhancement after MD and diazepam (DZ) treatment of mice raised in (A) SC or (B) EE. Each line represents one animal. The visual acuity of the open eye increased significantly in both groups.

Contrast sensitivity of the open eye was also significantly increased after MD in SC-mice $(n=6)$ and in EE- $(n=9)$ treated with diazepam (in both groups: $P<0.001$ at all analyzed frequencies, day 0 versus day 7, Bonferroni-adjusted t-test) (Figure 68). Values after 7 days of MD were also significantly higher compared to SC- and EE-mice without MD (for all measured frequencies: SC versus SC+MD+DZ: P < 0.01-0.001, Bonferroni-adjusted t-test; $\mathrm{EE}_{\mathrm{PD} 220}$ versus EE+MD+DZ: $\mathrm{P}<0.01-0.001$, Bonferroni-adjusted t-test) (Figure 67). After 7 days of $\mathrm{MD}$, contrast sensitivity was indistinguishable between $\mathrm{EE}_{\mathrm{PD} 220}$-mice and EE-mice with diazepam treatment ( $\mathrm{P}>0.05$, Bonferroni-adjusted t-test) (Figure 67). A slight, but significant difference of the contrast sensitivity of the open eye after MD was observed at some spatial frequencies between SC-mice and SC-mice with diazepam treatment, as indicated in (Figure 67). Values for contrast sensitivity of the measured spatial frequencies can be found in Table 8 . 


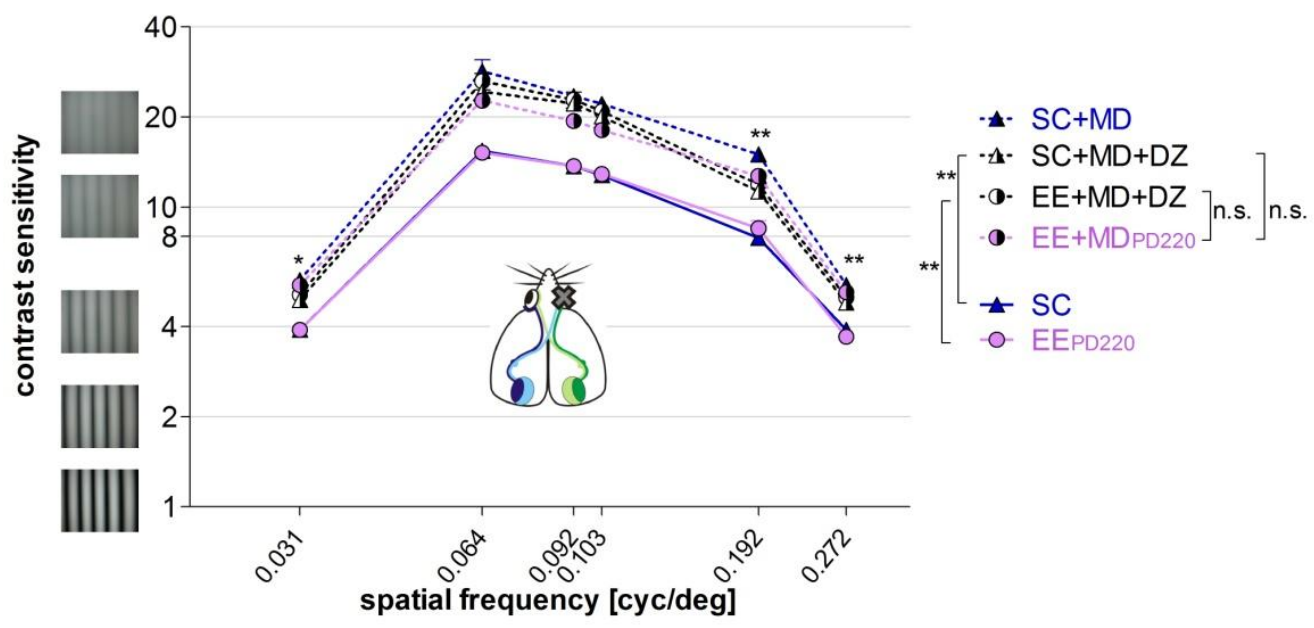

Figure 67: Contrast sensitivity of the open eye after MD increased significantly in diazepam (DZ) treated mice SC- and EE-mice. Contrast sensitivity was significantly higher in SC- and EEmice treated with diazepam compared to mice without MD (indicated is the minimal significance at all measured frequencies). EE-mice treated with diazepam were not significantly different to untreated EE-mice (PD 220) after MD. A slight difference was observed between diazepam treated and untreated SC-mice after MD, as indicated by the asterisk $\left({ }^{*}\right)$ symbols over the curves at the respective spatial frequency. 

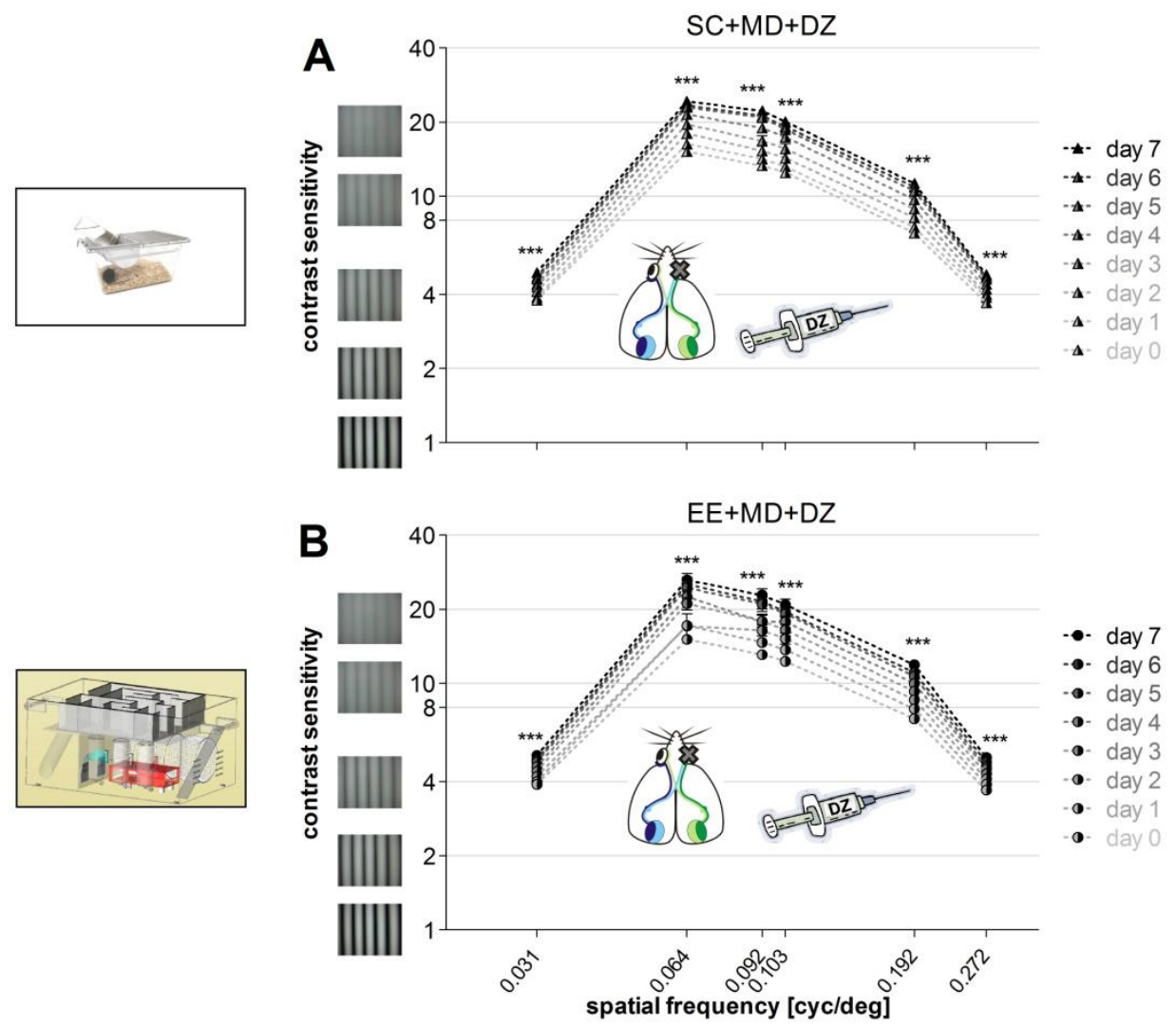

Figure 68: Mean contrast sensitivities for the individual days and spatial frequencies of mice raised in (A) SC or (B) EE and treated with diazepam (DZ) during the MD-period. Contrast sensitivity increased significantly in both groups after MD.

Table 8: Contrast sensitivity of the open eye increased significantly in mice raised in EE and SC and treated with diazepam (DZ) during MD. Average contrast values \pm S.E.M. on day 0 and day 7 after MD and statistical significance for the increase over these days (Bonferroni-adjusted t-test).

\begin{tabular}{|c|c|c|c|}
\hline Frequencies [cyc/deg] & Contrast sensitivity day 0 & Contrast sensitivity day 7 & $P$ \\
\hline EE+DZ+MD & & & \\
0.031 & $3.9 \pm 0.05$ & $5.1 \pm 0.09$ & $<0.001$ \\
0.064 & $15.1 \pm 0.69$ & $26.3 \pm 1.67$ & $<0.001$ \\
0.092 & $13.1 \pm 0.52$ & $22.8 \pm 1.41$ & $<0.001$ \\
0.103 & $12.3 \pm 0.54$ & $20.9 \pm 1.13$ & $<0.001$ \\
0.192 & $7.2 \pm 0.27$ & $11.9 \pm 0.47$ & $<0.001$ \\
0.272 & $3.7 \pm 0.08$ & $5.0 \pm 0.16$ & $<0.001$ \\
\hline SC+DZ+MD & & & \\
0.031 & $3.8 \pm 0.02$ & $4.9 \pm 0.10$ & $<0.001$ \\
0.064 & $15.2 \pm 0.23$ & $24.3 \pm 0.77$ & $<0.001$ \\
0.092 & $13.3 \pm 0.18$ & $22.2 \pm 0.77$ & $<0.001$ \\
0.103 & $12.5 \pm 0.11$ & $20.1 \pm 0.83$ & $<0.001$ \\
0.192 & $7.1 \pm 0.14$ & $11.3 \pm 0.48$ & $<0.001$ \\
0.272 & $3.7 \pm 0.02$ & $4.8 \pm 0.08$ & $<0.001$ \\
\hline
\end{tabular}




\subsubsection{EE-rearing did not affect the number of parvalbumin-positive cells and perineuronal} $\underline{\text { nets }}$

To find out whether a change in the number of PV-positive inhibitory neurons or PNNs could account for the prolonged sensitive phase for PD-plasticity we quantified them in V1 by means of immunofluorescence. Coronal brain sections $(40 \mu \mathrm{m})$ that included V1 were used. 4 sections per mouse and 4 mice per group (SC-mice PD 235 and EE-mice PD 220, both without MD) were analyzed. Triple immunofluorescence staining was performed for PV (Cy2 labeling), PNN staining with WFA- (Wisteria floribunda agglutinin, Cy3 labeling) and DAPI (4'.6-Diamidin-2-phenylindol, to visualize all cell nuclei and visualize cortical layers). All positively labeled PV-cells and PNNS were counted in V1 (layers II-VI) in a threedimensional tissue stack spanning the full thickness of the tissue.

Since inhibition by PVergic interneurons has been identified as an important player for ODplasticity in the adult brain (for recent reviews see Bavelier et al. 2010; Baroncelli et al. 2011), we visualized and quantified PV-positive neurons in V1. Clearly labeled PV-positive cells were found throughout the analyzed layers II-VI in V1 of SC- and EE-mice. This can be seen in the PV staining overview in Figure 69. The overview of PV-positive cells in all layers as well as a close-up already suggests that the numbers of PV-positive cells were not different between SC- and EE-mice (Figure 69). The quantification verified this impression: the number of PV-positive cells was with $5683.39 \pm 562.95$ cells $/ \mathrm{mm}^{3}(\mathrm{n}=4$ mice) in SC-mice not significantly different from EE-mice with $6396.47 \pm 277.76$ cells $/ \mathrm{mm}^{3} \quad(\mathrm{n}=4$ mice $)$ $(\mathrm{P}>0.05$, t-test; Figure 69).

Moreover, since the degradation of PNNs has also been shown to play a role in the enhancement adult of OD-plasticity (Pizzorusso et al. 2002) and reduced PNN-density was reported after EE-housing (Sale et al. 2007), we quantified WFA- positive labeled PNNs in V1. WFA-positive PNNs were found in all analyzed layers (II-VI) (Figure 69 - staining overview). At a higher magnification the staining revealed lattice-like structures around cells, often around PV-positive cells (Figure 69 - close up). The staining pictures already gave the impression of an unchanged number of WFA-positive PNNs after EE raising. Indeed, the quantification revealed that there was no significant difference in the number of WFApositive PNNs between SC-mice $\left(6167.34 \pm 726.69 \mathrm{PNNs} / \mathrm{mm}^{3}, \mathrm{n}=4\right.$ mice $)$ and EE-mice $\left(6408.04 \pm 130.80 \mathrm{PNNs} / \mathrm{mm}^{3}, \mathrm{n}=4\right.$ mice $)(\mathrm{P}>0.05, \mathrm{t}$-test; Figure 69).

To summarize, the number of PV-positive cells and WFA-positive PNNs was not changed in mice raised in EE compared to SC-mice. 


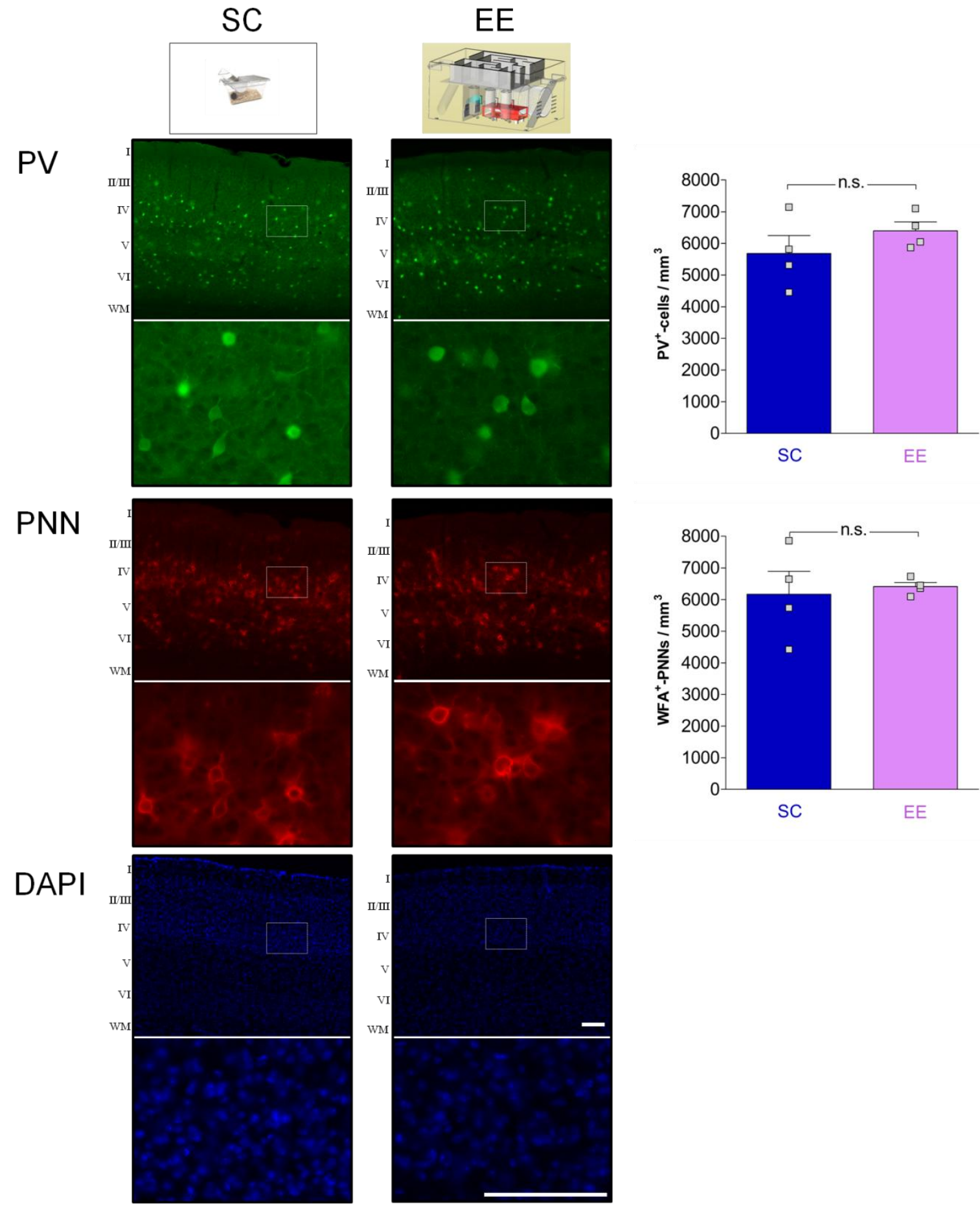

Figure 69: Number of PV-positive and WFA-positive PNNs in V1 was not different between mice raised in SC or EE. On the left side a representative example of a SC- and EE-mouse with the fluorescent triple staining of PV-positive cells (Cy2), WFA-positive PNNs (Cy3) and cell nuclei (DAPI) in the same section is illustrated. Included is an overview of layer I-VI with the underlying white matter (WM) and a close up of the area marked by the white square. On the right side a quantification of the number of PV-positive cells and WFA-positive PNNs per $\mathrm{mm}^{3}$ in V1 (layer IIVI) are shown. Both of them were not significantly different between SC- and EE-mice. Squares symbolize the mean value of the 4 mice analyzed in each group. Scale bars: $100 \mu \mathrm{m}$. 


\subsection{Can enriched environment protect from stroke impairments?}

As described in the first part of the results chapter, a PT-lesion abolished the enhancement of vision after MD (chapter 3.1.2, starting page 56) and impaired OD-plasticity (chapter 3.1.3, starting page 61). We showed that EE could prolong and restore OD-plasticity in adult mice (chapter 3.4.1, starting page 93). Therefore the question arose whether EE, as a non-invasive procedure, could also prevent the loss of OD-plasticity in PT-lesioned mice. To this end we raised mice in EE until PD 90 and then exposed them to a stroke. ODIs were acquired from the left (lesioned) hemisphere. The mice were between PD 80 and 114 at the day of optical imaging. The contralateral eye was deprived for 7 days, as in the previous experiments.

\subsubsection{Localization and size of the photothrombotic lesion}

The cortical lesion was induced in the left hemisphere using the PT-technique as in the experiments described before (chapter 3.1.1, page 53). Lesion size and location was analyzed in Nissl-stained frontal section 7 days after PT. The center of the lesion was situated $1.0 \pm 0.12 \mathrm{~mm}$ anterior to $\mathrm{V} 1$ and $2.0 \pm 0.21 \mathrm{~mm}$ lateral to the midline (Figure 70). It was $1.2 \pm 0.12 \mathrm{~mm}$ posterior to the bregma in the primary somatosensory cortex (S1), which corresponds primarily to the primarily hind limb and shoulder region (Paxinos \& Franklin 2001). The lesion diameter was $0.6 \pm 0.10 \mathrm{~mm}$ medio-laterally and $0.6 \pm 0.08 \mathrm{~mm}$ anterioposteriorly. The lesion location and size for the mentioned parameters was not different to the lesions of the other experimental groups described before $(\mathrm{P}>0.05$, Bonferroni adjusted $\mathrm{t}-$ test; see also chapter 3.1.1, page 53), except for the medio-lateral diameter, which was smaller in the EE-experiments $(\mathrm{P}<0.01$, Bonferroni-adjusted t-test $)$. 


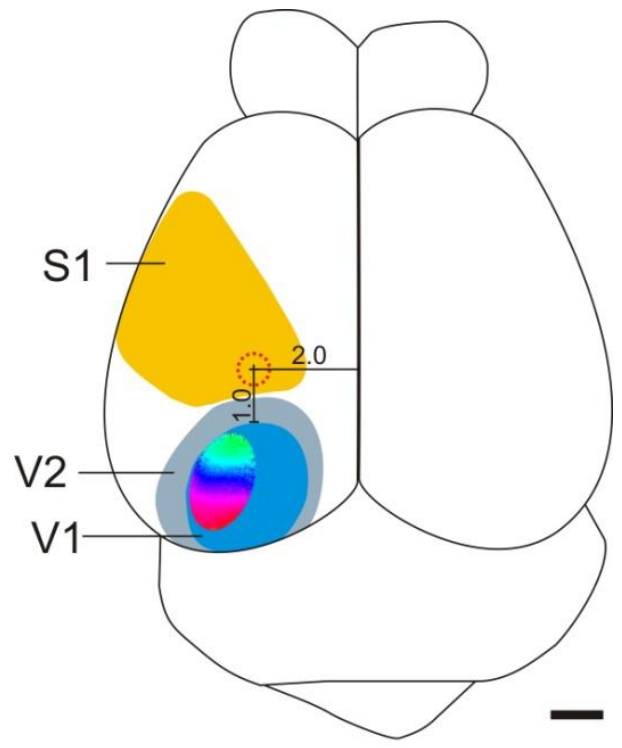

Figure 70: Location and size of the PT-lesion of mice raised in EE. Scheme of a mouse brain with a photothrombotic lesion (red dotted circle) showing the average lesion size and location. The lesion center was located in the left S1, on average $1.0 \mathrm{~mm}$ anterior to the anterior border of V1 and $2.0 \mathrm{~mm}$ lateral to the midline. A retinotopic map from the binocular part of $\mathrm{V} 1$ acquired with optical imaging of intrinsic signals was interposed to visualize the spatial relation of the lesion and V1. Scale bar: $1 \mathrm{~mm}$.

\subsubsection{EE preserved OD-plasticity after a photothrombotic lesion}

In SC-mice OD-plasticity was abolished after a PT-lesion (see chapter 3.1.3, page 61). In contrast to that, a pronounced OD-shift towards the open eye could be induced in PT-mice raised in EE.

In the optically imaged response magnitude maps of the binocular region of V1, PT-lesioned EE-mice without MD showed the typical contralateral dominance: the activity patches evoked by stimulation of the contralateral eye were darker than those after stimulation of the ipsilateral eye, the histogram of ODIs was centered right of zero and the two-dimensional OD-map displayed warm red colors and the ODI was positive (Figure $71 \mathrm{~A}$ ). The ODI was positive $(0.24 \pm 0.01, \mathrm{n}=4)$ and not significantly different to PT mice raised in SC or EEmice without PT $(\mathrm{P}>0.05$, Bonferroni-adjusted t-test $)$.

After MD, the lesioned EE-mice displayed an obvious OD-shift toward the open eye: the response magnitude maps after stimulation of the ipsilateral (open) eye were even slightly darker then after contralateral (deprived) eye stimulation, the histogram of OD-scores shifted to the left, colder colors prevailed in the OD-map and the ODI was negative (Figure $71 \mathrm{~B}$ ). The ODI decreased significantly to $0.02 \pm 0.04(\mathrm{n}=5)$ after 7 days of $\mathrm{MD}(\mathrm{P}<0.01$, 
Bonferroni-adjusted t-test) (Figure $72 \mathrm{~A}$ ). The ODI after MD was significantly lower than in SC-mice with PT after MD ( $\mathrm{P}<0.01$, Bonferroni-adjusted t-test) and not significantly different to EE-mice after MD (P > 0.05, Bonferroni-adjusted t-test).

The OD-shift in EE-mice with PT was even juvenile-like: after MD contralateral (deprived) eye responses were significantly reduced (without MD: $1.69 \pm 0.09, \mathrm{n}=4$; with MD: $1.21 \pm 0.15, \mathrm{n}=5 ; \mathrm{P}<0.05, \mathrm{t}$-test), whereas the ipsilateral open eye responses remained unchanged (without MD: $1.04 \pm 0.06$; with MD: $1.19 \pm 0.12, \mathrm{P}>0.05$, t-test) (Figure $72 \mathrm{~B}$ ). After MD response strength of the two eyes were no longer significantly different $(\mathrm{P}>0.05, \mathrm{t}-$ test) (Figure $72 \mathrm{~B}$ ). Interestingly, the response magnitude in EE-mice with PT was significantly smaller than in SC-mice with PT: in animals without MD maximum responses after ipsilateral eye stimulation and also pooled maximum responses for ipsi- and contralateral eye stimulation were significantly different $(\mathrm{P}>0.05$, t-test) (Figure $72 \mathrm{~B}$ ).

Consequently, EE-housing could prevent the loss of OD-plasticity after a PT-lesion. Mice showed even a juvenile-like form of OD-plasticity. 


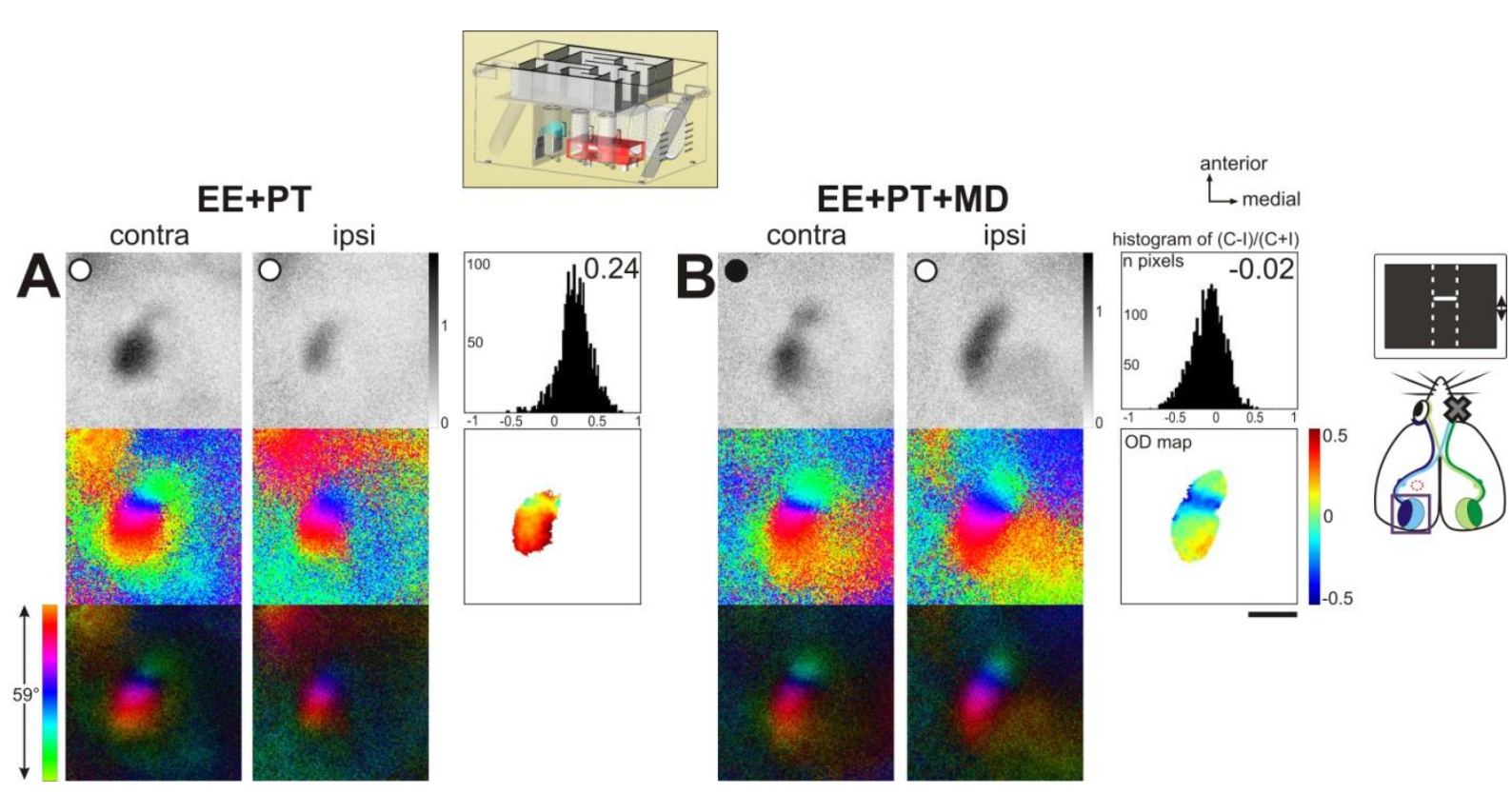

Figure 71: EE preserved OD-plasticity after a PT-lesion. Optical imaging maps of responses to contra- and ipsilateral eye stimulation in the binocular region of mouse visual cortex in PD 90 mice raised in EE with a PT-lesion without MD (A) and with MD of the contralateral eye (B). Gray-scale coded response magnitude maps and their quantification (top), and color-coded phase and polar maps of retinotopy (middle, bottom) are illustrated. The magnitude of the optical responses is illustrated as fractional change in reflection $\times 10^{4}$. Black filled circles on the response magnitude maps indicate the deprived eye, unfilled circles non-deprived eyes. For each experiment, the histogram of OD-scores, the average OD-index (ODI), and the corresponding two-dimensional OD-maps are included (ODI values are color-coded: blue represents negative, red positive values). (A) Without MD mice raised in EE that received a PT-lesion showed the typical contralateral dominance: activity patches evoked by stimulation of the contralateral eye were consistently darker than those after stimulation of the ipsilateral eye, two-dimensional OD-maps displayed warm colors and the average ODI was positive. (B) After MD, an obvious OD-shift could be observed: the response magnitude maps after stimulation of the ipsilateral (open) eye were even slightly darker then after contralateral (deprived) eye stimulation, the histogram of OD-scores shifted to the left, the ODI was even negative and colder colors prevailed in the OD-map. Scale bar: $1 \mathrm{~mm}$. 


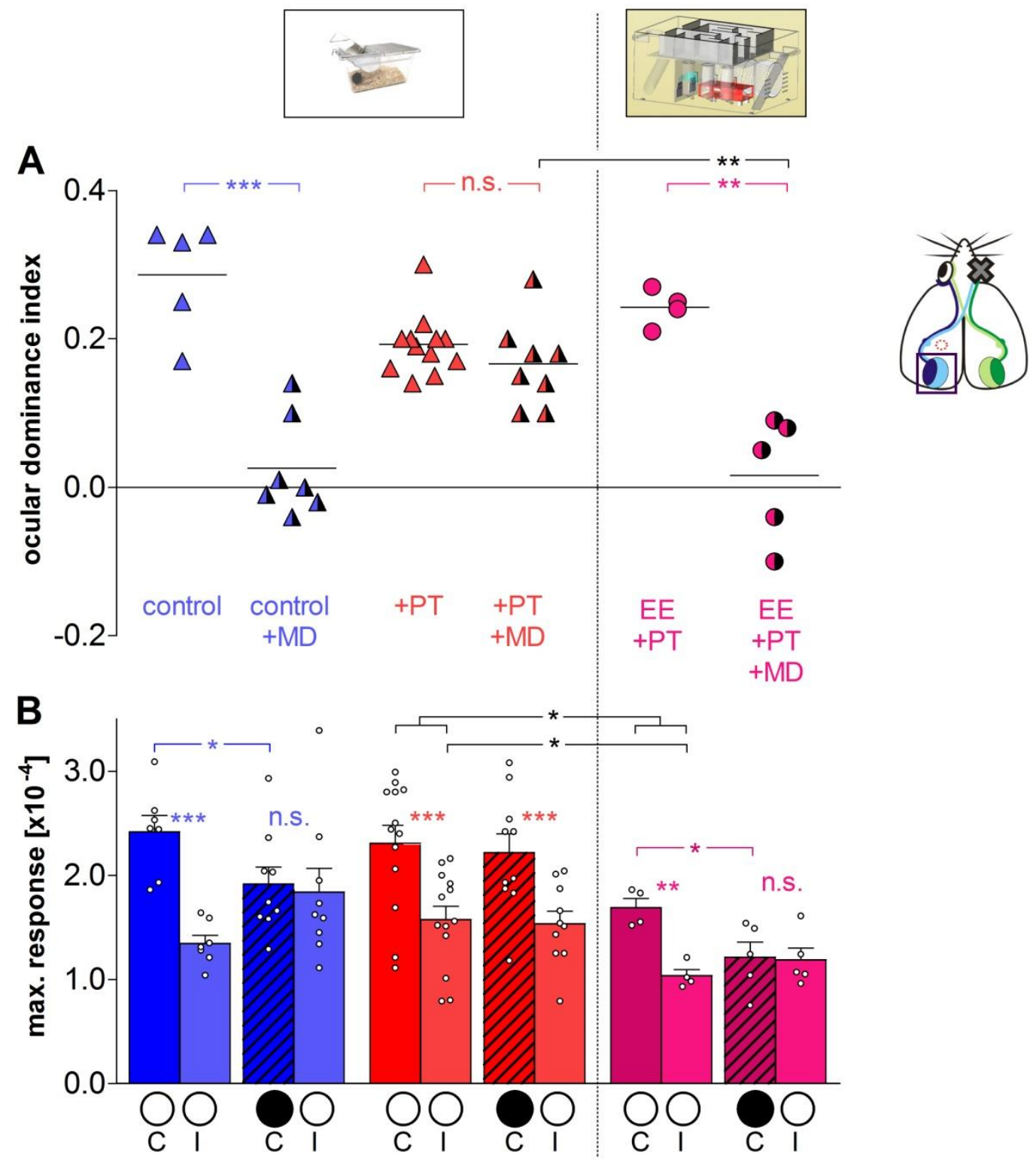

Figure 72: EE preserved OD-plasticity after a PT-lesion. Optical imaging data of the left hemisphere (see inset) (A) In control animals, MD induced a significant reduction of the ODI (control+MD) whereas in PT-animals raised in SC, ODIs were not changed after MD. In contrast to that, when mice were raised in EE the loss of OD-plasticity due to a PT-lesion could be prevented and a strong OD-shift could be induced. (B) Maximum response magnitude in V1 elicited by stimulation of the contralateral (C) or ipsilateral (I) eye. Black filled circles represent deprived eyes, unfilled circles non-deprived eyes. In control animals, cortical activation after stimulation of the contralateral eye was significantly higher than after ipsilateral eye stimulation, reflecting the contralateral dominance. After MD of the contralateral eye, response strengths of the two eyes were no longer significantly different, whereas, after MD in PT-animals, the response strengths of both eyes remained significantly different. When mice were raised in the EE a juvenile-like form of OD-plasticity could be still induced in PT-mice: deprived eye responses decreased significantly and response strengths of the two eyes were no longer significantly different. Moreover, the response magnitude in EE-mice with PT was significantly smaller than in SC-mice with PT. 


\subsubsection{EE partially preserved enhancement of vision after MD}

The visual acuity of EE-mice with a PT-lesion was $0.38 \pm 0.002 \mathrm{cyc} / \mathrm{deg}$, and remained stable over 7 days (day 7: $0.38 \pm 0.002$ cyc/deg, $\mathrm{n}=8$; $\mathrm{P}>0.05$, Bonferroni-adjusted t-test) (Figure 73 , Figure $74 \mathrm{~A})$. This was not significantly different to EE-mice without PT (EE $E_{\mathrm{PD} 220}: \mathrm{F}_{1,12}=$ 0.06, $\mathrm{P}>0$., ANOVA). In contrast to PT-mice raised in SC, which had no increase in the visual acuity after MD (see chapter 3.1.2, page 56), in PT-mice raised in EE the visual acuity increased significantly from $0.38 \pm 0.002 \mathrm{cyc} / \mathrm{deg}$ on day 0 (before MD) to $0.42 \pm 0.006 \mathrm{cyc} / \mathrm{deg}$ after 7 days of $\mathrm{MD}(=10.0 \pm 1.7 \%, \mathrm{n}=8 ; \mathrm{P}<0.05$, Bonferroniadjusted t-test) (Figure 73, Figure $74 \mathrm{~B}$ ). This was significantly different to EE+PT-mice without $\mathrm{MD}\left(\mathrm{F}_{1,14}=47.58, \mathrm{P}<0.001\right.$, ANOVA) (Figure 73$)$. But the increase was still significantly lower than in SC-control mice with MD (day 1 to day 7: $21.6 \pm 1.4 \% ; \mathrm{F}_{1,16}=$ 16.95, $\mathrm{P}<0.01$, ANOVA $)$ and in EE-mice with $\mathrm{MD}\left(\mathrm{EE}_{\mathrm{PD} 220}: \mathrm{F}_{1,12}=14.30, \mathrm{P}<0.05\right.$, ANOVA).

Consequently, EE partially preserved the enhancement of vision after MD.

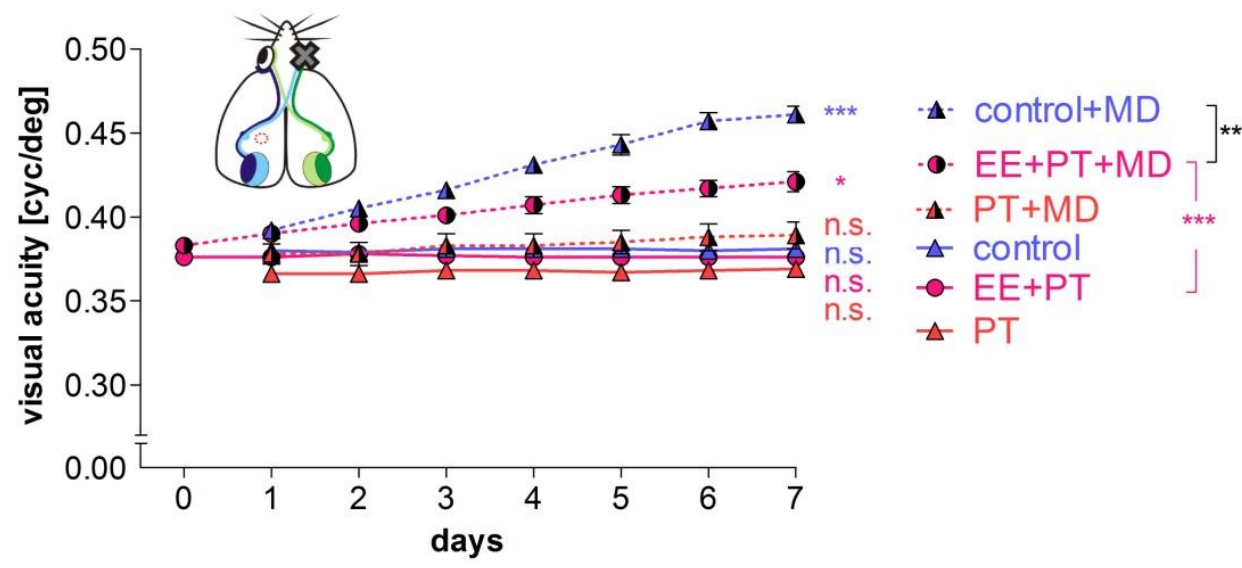

Figure 73: EE partially preserved visual acuity enhancement after MD in PT-mice. In mice with a PT-lesion that were raised in SC there was no significant increase of the visual acuity of the open eye after MD (PT+MD). In contrast to that, in mice raised in $\mathrm{EE}(\mathrm{EE}+\mathrm{PT}+\mathrm{MD})$ the visual acuity of the open eye after MD increased significantly but was still lower than in SC control mice after MD (control+MD). 

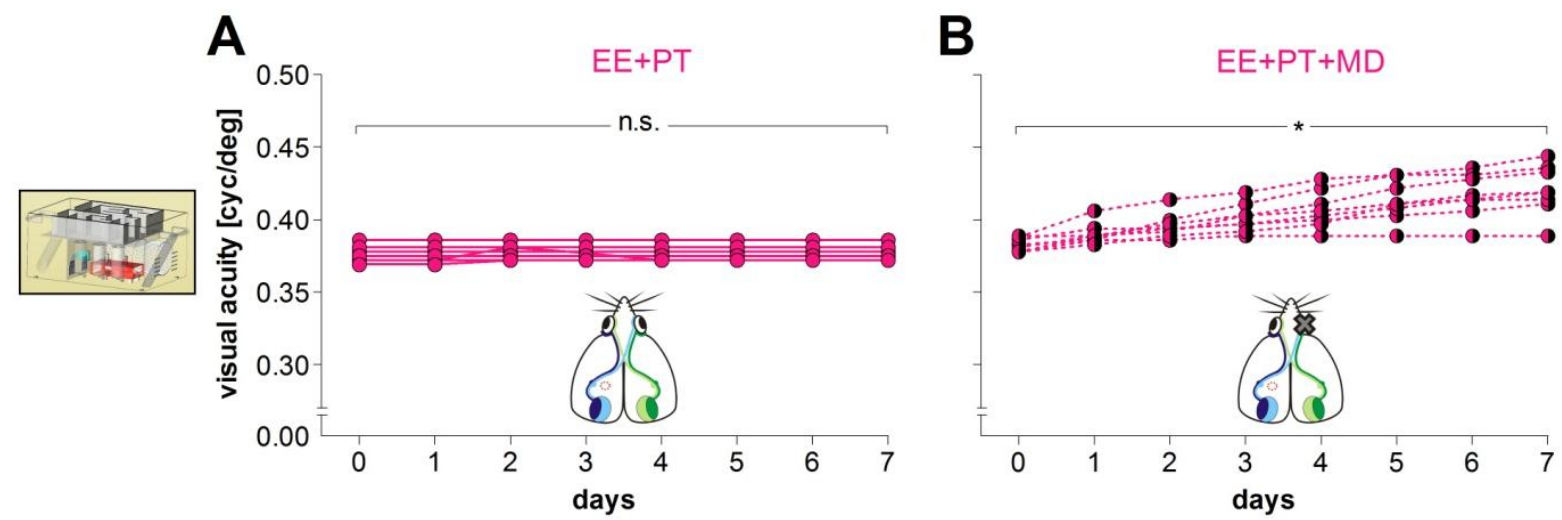

Figure 74: Individual values of mice with a PT-lesion, raised in EE. Each line represents one animal. (A) Without MD visual acuity remained stable over 7 days. (B) After 7 days of MD visual acuity of the open eye increased significantly.

Likewise, the contrast sensitivity of the open eye after MD increased significantly in mice raised in EE despite the PT-lesion ( $\mathrm{n}=8, \mathrm{P}<0.001$ for all measured frequencies, Bonferroniadjusted t-test) (Figure $76 \mathrm{~B}$, for values see Table 9), so that the contrast sensitivity after MD was significantly higher than in the EE with PT-group without MD (at least $\mathrm{P}<0.05$ for all measured frequencies, Bonferroni-adjusted t-test) (Figure 75). In PT-animals raised in EE without MD contrast sensitivity remained stable over 7 days (at $0.064 \mathrm{cyc} / \mathrm{deg}$, day 0 : $12.0 \pm 1.73, \bumpeq 8 \%$ contrast, day $7: 12.1 \pm 1.73, \bumpeq 8 \%$ contrast, $\mathrm{n}=9 ; \mathrm{P}>0.05$ for all measured frequencies, Bonferroni-adjusted t-test) (Figure $76 \mathrm{~A}$ ). However, the contrast sensitivity after MD in PT-mice raised in EE was at some spatial frequencies still lower than in control mice raised in a SC (for statistical significance see Figure 75).

Concluding, raising mice in EE partially preserved sensory learning after a PT-stroke lesion. 


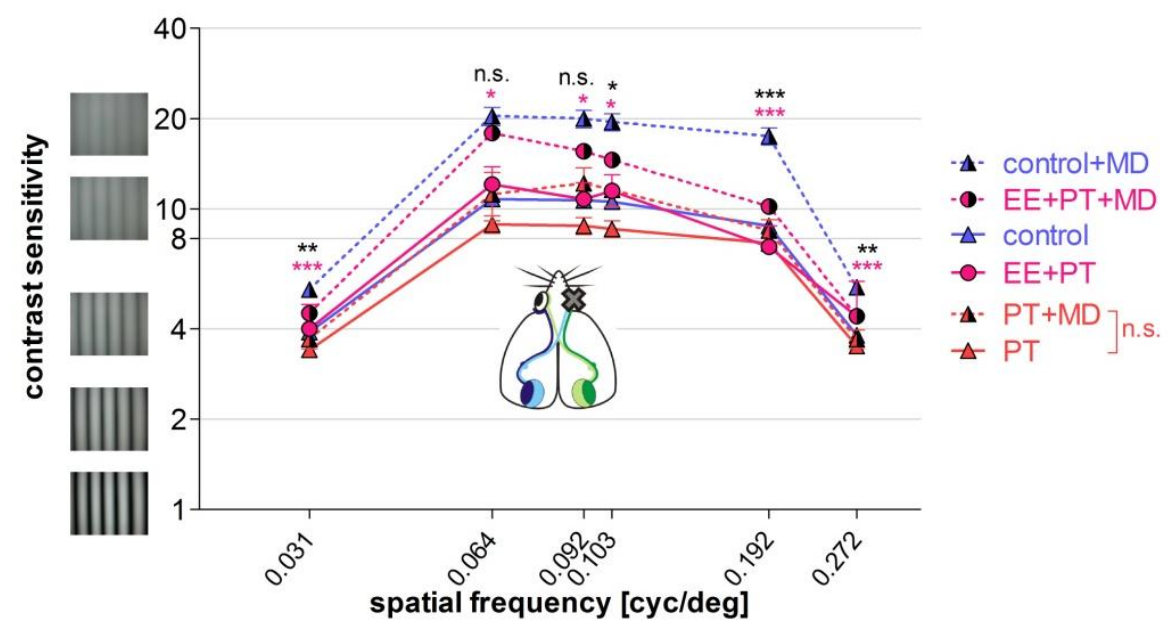

Figure 75: EE partially preserved increase of contrast sensitivity after MD in PT-mice. Contrast sensitivity values on days 7. In mice with a PT-lesion that were raised in SC, there was no significant increase of the visual acuity of the open eye after MD (PT+MD). In contrast to that, in mice raised in $\mathrm{EE}(\mathrm{EE}+\mathrm{PT}+\mathrm{MD})$ the contrast sensitivity of the open eye increased significantly after MD (statistical significance in comparison to EE+PT without MD is indicated by pink asterisk (*) symbols), but was at some measured frequencies still lower than in control mice after MD (control+MD) (statistical significance indicated in black).
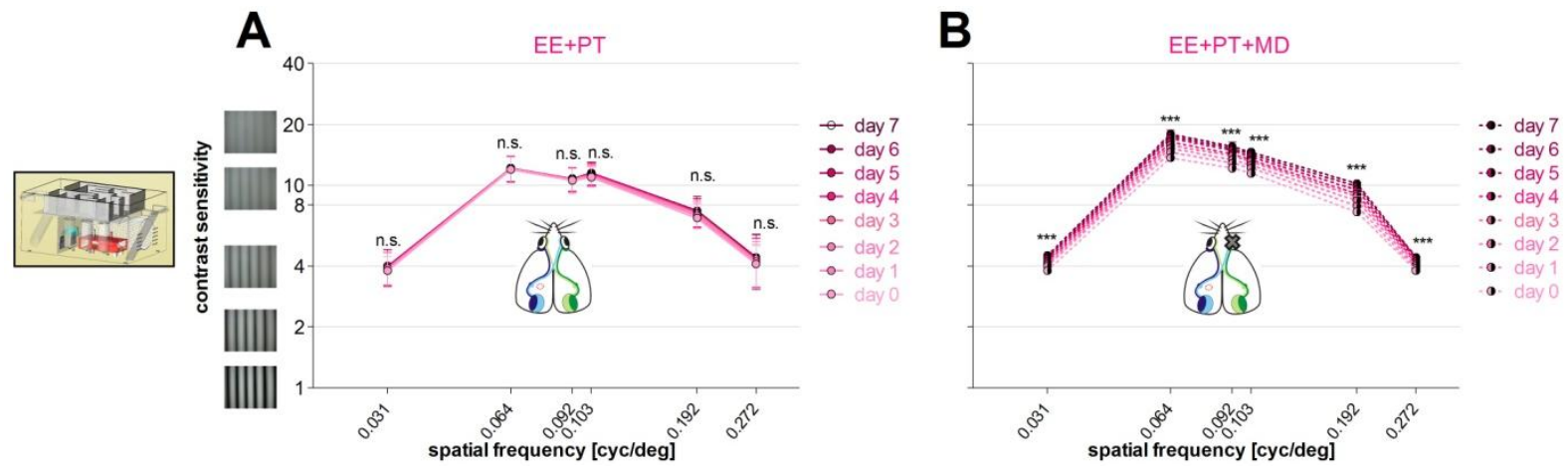

Figure 76: Mean contrast sensitivities for the individual days and spatial frequencies of PT-mice raised in EE. (A) In animals without MD contrast sensitivity remained stable over 7 days. (B) After MD the contrast sensitivity of the open eye increased significantly at all measured spatial frequencies.

Table 9: Contrast sensitivity of the open eye increased significantly in PT-mice raised in EE after MD. Average contrast values \pm S.E.M. on day 0 and day 7 after MD and statistical significance for the increase over these days (Bonferroni-adjusted t-test).

\begin{tabular}{|c|c|c|c|}
\hline Frequencies $[\mathrm{cyc} / \mathrm{deg}]$ & Contrast sensitivity day 0 & Contrast sensitivity day 7 & $\mathrm{P}$ \\
\hline $\mathrm{EE}+\mathrm{PT}+\mathrm{MD}$ & & & \\
\hline 0.031 & $3.8 \pm 0.12$ & $4.5 \pm 0.14$ & $<0.001$ \\
0.064 & $13.7 \pm 0.52$ & $17.9 \pm 0.87$ & $<0.001$ \\
0.092 & $12.2 \pm 0.34$ & $15.6 \pm 0.76$ & $<0.001$ \\
0.103 & $11.5 \pm 0.29$ & $14.6 \pm 0.64$ & $<0.001$ \\
0.192 & $7.4 \pm 0.26$ & $10.2 \pm 0.39$ & $<0.001$ \\
0.272 & $3.8 \pm 0.11$ & $4.4 \pm 0.12$ & $<0.001$ \\
\hline
\end{tabular}




\section{DISCUSSION}

\subsection{Summary of the main results}

Plasticity of the brain is crucial for rehabilitation after stroke. In the present study, we investigated the influence of a photothrombotically induced cortical lesion (PT) in the primary somatosensory cortex (S1) on ocular dominance (OD) plasticity in the neighboring primary visual cortex (V1) in vivo, using optical imaging of intrinsic signals (Kalatsky \& Stryker 2003). Moreover, in the same mice we analyzed sensory learning, i.e. the increase of visual acuity and contrast sensitivity after monocular deprivation (MD), using a behavioral test: the optomotor system (Prusky et al. 2004). In control animals, 7 days of MD induced a significant increase of visual abilities of the open eye and an OD-shift towards this eye. After a PT-lesion visual plasticity was abolished: there was neither an enhancement of visual abilities nor an OD-shift in the lesioned hemisphere after MD. Although sensory learning was compromised, irrespective of whether the deprived eye was ipsi- or contralateral to the lesioned hemisphere, OD-plasticity was still present in the non-lesioned hemisphere. To test whether antiinflammatory treatment or a time delay between lesion induction and MD could restore plasticity, we treated the mice with the anti-inflammatory drug ibuprofen or induced a delay of 2 weeks between PT and MD. Indeed, in both conditions the sensory learning was restored after MD, while OD-plasticity was still absent.

Enriched environment (EE) housing is a non-invasive strategy that can enhance plasticity mechanisms (e.g. Sale et al. (2007)). We tested the influence of EE-housing in non-lesioned mice and in mice with a stroke lesion. Indeed, in non-lesioned mice EE was capable of prolonging OD-plasticity into late adulthood. Mice that were raised in EE showed still ODplasticity up to 196 days, in contrast to mice raised in standard cages (SC), where ODplasticity was not inducible any more beyond postnatal day (PD) 110 (Lehmann \& Löwel 2008). Moreover, the OD-shift in adult EE-mice was juvenile-like: it was mediated by a decrease in deprived eye responses (Hofer et al. 2006b; Heimel et al. 2007). Next, we investigated if reduced inhibition could be one of the mechanisms involved in prolonged ODplasticity after EE raising. Diazepam treatment, which should increase GABAergic $(\gamma$ aminobutyric acid) inhibition, did not completely abolish the OD-shift in EE-mice. The number of parvalbumin (PV) positive interneurons, which are the major GABAergic cells in the visual cortex of mice (Gonchar et al. 2007), and perineuronal nets (PNNs) was not changed after EE raising. Next we investigated if EE-housing also is capable of restoring 
already lost plasticity. In fact, when SC-mice were transferred to EE at PD 110 (late EE), ODplasticity was restored even up to PD 320. EE also revealed a promising effect in mice with a PT stroke lesion: raising mice in EE could preserve OD-plasticity and partly preserve sensory learning after MD.

The main results of our study in terms of OD-plasticity are summarized in Figure 77 and in terms of enhancement of vision after MD ("sensory learning") in Figure 78. Some parts of the discussion concerning the stroke study (chapter 4.2-0) are also presented, in a different version, in our published paper (Greifzu et al. 2011).

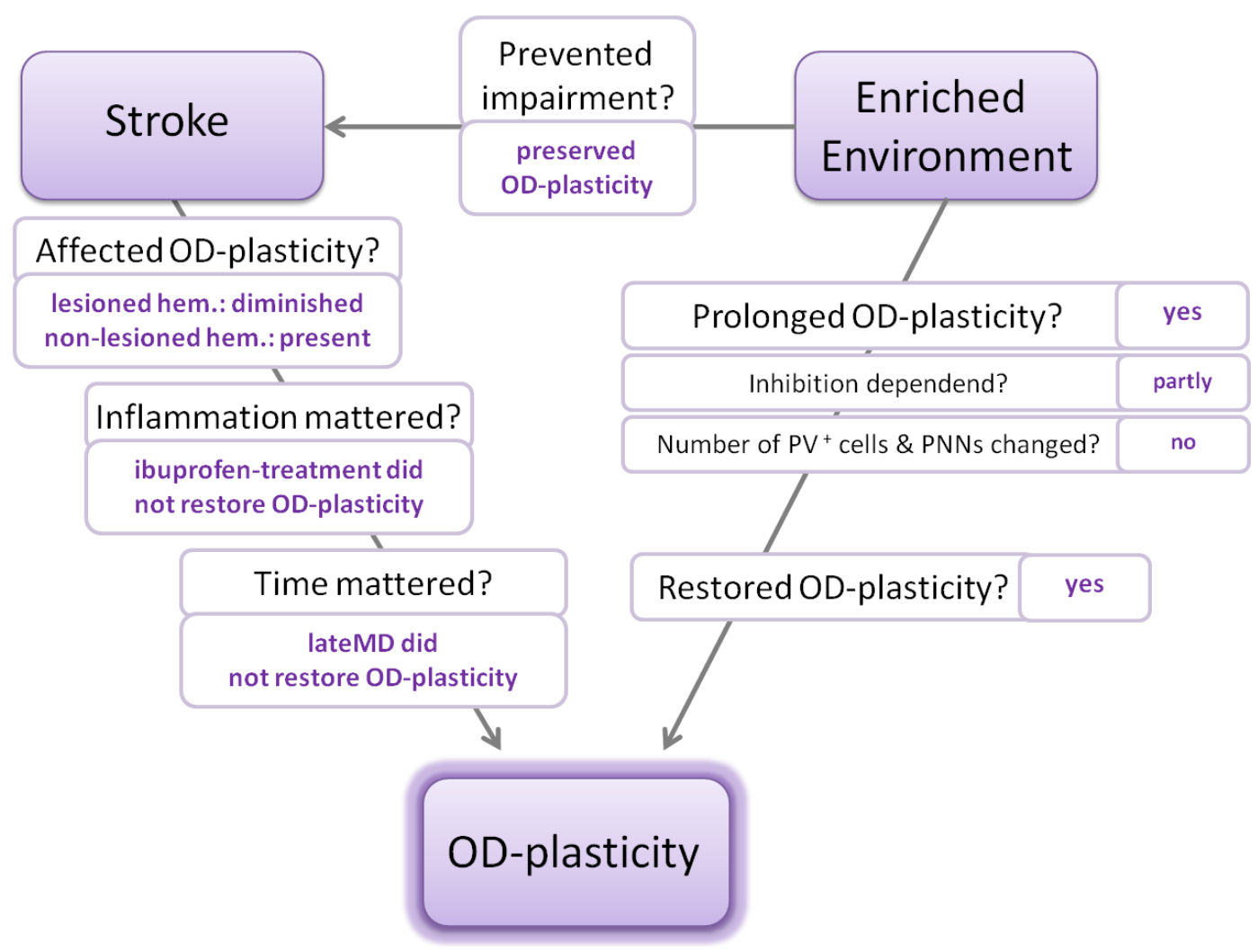

Figure 77: Overview scheme of the main findings of the thesis regarding OD-plasticity. ODplasticity induced by MD was examined using optical imaging of intrinsic signals in an in vivo approach in mice. We observed that a focal photothrombotically induced cortical stroke in the somatosensory cortex diminished OD-plasticity in the neighboring visual cortex in the lesioned hemisphere (hem.), but OD-plasticity was still present in the non-lesioned hemisphere. Treatment with ibuprofen to counteract the inflammatory reaction after the stroke did not restore OD-plasticity. A two-week delay of the MD after lesioning did also not restore OD-plasticity in the lesioned hemisphere. As another therapeutic option to improve plasticity we used EE (on the right). Raising mice in EE prolonged OD-plasticity into late adulthood, even in a juvenile-like form. This prolongation was only partly abolished by diazepam treatment, which should increase inhibition. Using immunofluorescence we observed that the numbers of PV-positive labeled cells and WFApositive labeled PNNs in V1 were not different between EE- and SC-mice. Moreover, at an age when OD-plasticity is not inducible any more in SC-mice, it could be restored by housing mice in the EE. Impressively, EE could also prevent impairment of OD-plasticity after a cortical stroke (top). 


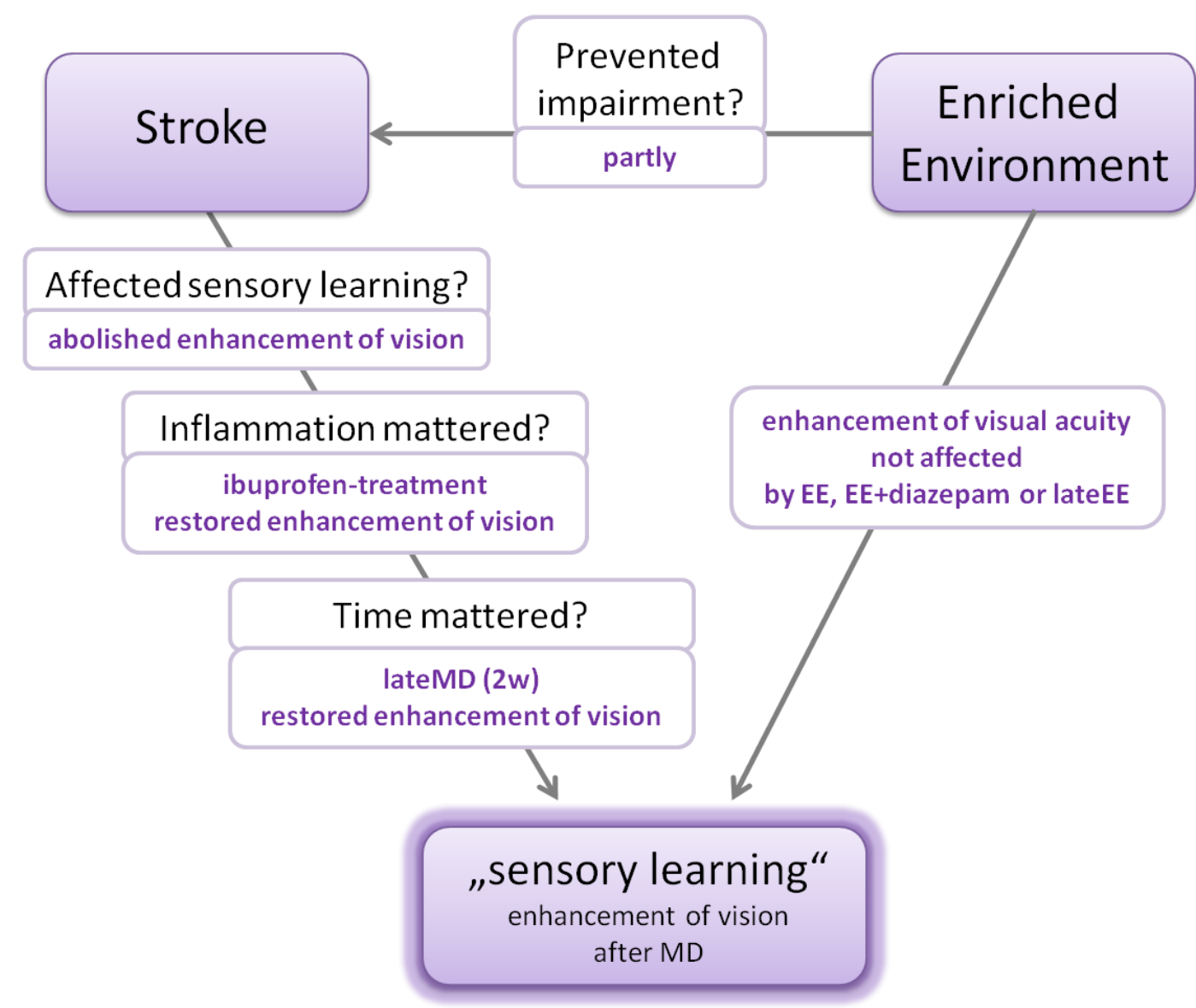

Figure 78: Overview scheme of the main findings of the thesis regarding the enhancement of vision after MD ("sensory learning"). Sensory learning, as a form of plasticity, was measured using a behavioral test in the virtual reality optomotor system for measuring visual acuity and contrast sensitivity (optokinetic response) in mice. We observed that a photothrombotic cortical stroke in the somatosensory cortex abolished enhancement of vision after MD (left). Treatment with the antiinflammatory drug ibuprofen completely restored sensory learning. A 2 weeks ( $2 \mathrm{w}$ ) delay of the MD after lesioning also restored enhancement of vision of the open eye completely. EE (right) did not affect the enhancement of visual acuity after MD. Impressively, EE-housing from birth could partially prevent the impairment of sensory learning after a cortical stroke (top).

\subsection{Inflammation, time and treatment}

In our experiments, sensory learning after MD was abolished in mice with a PT-stroke. Stroke initiates an inflammatory reaction at the injury site and surrounding areas (Garcia 1975; Liu et al. 1994; Sairanen et al. 2001; Schroeter et al. 2002) (for a review, see Wang et al. 2007; Liguz-Lecznar \& Kossut 2013). In order to counteract this inflammation and restore visual plasticity we tested the therapeutic effect of the non-selective cyclooxygenase- (COX) inhibitor ibuprofen (Mitchell et al. 1993), which can cross the blood-brain barrier (Parepally et al. 2006). COX-2 is a pro-inflammatory enzyme that is rapidly induced in inflamed tissues and participates in glutamate excitotoxicity and cytotoxicity associated with inflammation after stroke (Bidmon et al. 2000; review: Cherubini et al. 2005). In our experiments, ibuprofen 
treatment completely rescued the compromised sensory learning after a PT-lesion, but had no effect on the compromised OD-plasticity. In the ischemic cortex, levels of pro-inflammatory mediators increase about 1 hour after stroke, and return to basal values after 5 days (Liu et al. 1993; Wang et al. 1994). To analyze whether the inhibited plasticity in our study was an acute effect or a longer lasting modification of neuronal circuits, we inserted a delay between the PT-lesion and MD. With a delay of 2 weeks after the PT-lesion, sensory learning after MD was restored to control values, as after anti-inflammatory treatment, but OD-plasticity in the lesioned hemisphere remained absent. Hence, the reduced sensory learning after a PT-lesion was an acute effect, most probably due to an inflammation.

Jablonka et al. (2012b) recently showed that ibuprofen treatment can restore experiencedependent plasticity after a PT-lesion in the neighboring barrel cortex in rats. The distance between the PT-lesion and the analyzed barrel field was similar to the distance from the PTlesion to V1 in our experiments. Plasticity of cortical representation was induced by trimming all whiskers except for one row for 1 month, and cortical representation was visualized with $\left[{ }^{14} \mathrm{C}\right]$-2-deoxyglucose autoradiography. In contrast to control animals, rats with a PT stroke showed no enlargement of functional cortical representation of the spared whisker row after stimulation. Daily injections of ibuprofen throughout the whisker deprivation period could restore the enlargement of functional cortical representation of the spared whisker row up to control values. After ibuprofen treatment in our PT-mice we found restored sensory learning after MD, but OD-plasticity could not be restored. When Jablonka et al. (2012b) initiated the whisker deprivation with a delay of 1 or 4 weeks after stroke, cortical plasticity in the barrel field was partially recovered at both times, although control values were not reached. In our study, a 2 week delay between PT and MD resulted in an increase of visual abilities up to control levels, but OD-plasticity was still diminished. Since OD-plasticity and plasticity in the barrel cortex after whisker trimming are both models of experience-dependent plasticity in the cortex (reviewed in Feldmann 2009), one would expect similar results in the PT-experiments. However there is a discrepancy between the effects of ibuprofen and late deprivation on cortical experience-dependent plasticity in the barrel cortex (plasticity at least partly restored) (Jablonka et al. 2012b) compared to our results in the visual cortex (OD-plasticity not restored) (Greifzu et al. 2011). This may be due to different factors. First of all, Jablonka et al. (2012b) started the ibuprofen treatment 30 minutes before stroke. Thus, this study shows rather a prevention of stroke impairment than rehabilitation. Our treatment started 4 to 5 hours after stroke in order to test the drug for a clinical setting. Starting our drug treatment also before the PT-lesion induction might also act as prevention and OD-plasticity might be 
persevered. Other factors for the diverging results might be: the experimental animals (rat vs. mice), cortical areas analyzed (primary somatosensory area versus primary visual area), different plasticity paradigms (whisker deprivation vs. MD) and different duration of deprivation (4 weeks for whiskers versus 1 week MD). The deprivation time of 4 weeks used by Jablonka et al. (2012b), together with the delay experiments adds up to 5 or 8 weeks respectively from the time of PT-lesion until the $\left[{ }^{14} \mathrm{C}\right]-2$-deoxyglucose autoradiography mapping of the spared whiskers. Thus, to observe whether the impaired OD-plasticity in our experiments would also be re-established at later time points, it would be interesting to examine OD-plasticity after a longer delay time (for example 4 weeks) between PT and MD. However, a limitation of the study of Jablonka et al. (2012b) is, that they used the nonlesioned hemisphere as a control and reference hemisphere to calculate the increase in cortical whisker representation. As mentioned before, the non-lesioned hemisphere is also quite likely to be affected by the PT-lesion and is therefore unsuitable as a control. Nevertheless, Jablonka et al. (2012a) started to examine the influence of interhemispheric interactions in their post stroke experience-dependent plasticity model and found a callosal effect, although it still remains unclear how it is mediated.

It has been shown that the monocular V1 on the hemisphere ipsilateral to the deprived eye is needed for the increase in visual acuity after MD (Prusky et al. 2006). In our experiments, this hemisphere was the non-lesioned "intact" hemisphere. However, sensory learning was abolished irrespective of whether the deprived eye was contra- or ipsilateral to the lesioned hemisphere. What does that tell us? Evidently there is no "intact" hemisphere, since even a small PT-lesion in the somatosensory cortex of the opposite hemisphere can abolish sensory learning, most probably mediated by a non-local inflammatory reaction affecting both hemispheres. The hypothesis that a non-local inflammation is responsible for diminished sensory learning is further supported by the result that neither lesion location nor size influenced the magnitude of impairment. Even animals with a very small lesion in the Nisslstained sections had impaired sensory learning.

To summarize, the impairment in sensory learning was most probably caused by a transient, but global inflammatory process. Anti-inflammatory treatment with ibuprofen might be a useful adjuvant therapy to support rehabilitation in the early post-stroke period.

Sensory learning versus OD-plasticity. Sensory learning could be restored after ibuprofen treatment and late MD, in contrast to OD-plasticity that was still impaired. How can that be explained? The different susceptibility to the treatment (restored plasticity versus not restored) 
suggests that the two forms of visual plasticity (sensory learning and OD-plasticity) are mediated by different neuronal networks. In fact, Prusky et al. (2006) observed, that the enhancement of visual acuity of the open eye after MD is restricted to the monocular segment of V1, while OD- shifts are the result of competition between the afferents of the two eyes for cortical territory that takes place in the binocular segment of V1 (Gordon \& Stryker 1996; Cang et al. 2005; Hofer et al. 2006a). Moreover, the increase in visual abilities involves the cortical control of the accessory optic system triggering the reflex (Prusky et al. 2006), presumably from deep-layer efferent projections, whereas OD-plasticity in animals beyond the critical period mostly occurs in the superficial cortical layers (Daw et al. 1992; Pham et al. 2004; Tagawa et al. 2005).

Conclusively, the different susceptibilities of sensory learning and OD-plasticity to ibuprofen treatment and late MD might be due to the fact that these two models of visual plasticity are mediated by different mechanisms and neuronal networks.

\subsection{Reduced or increased plasticity?}

In mice with a cortical PT-lesion we found diminished OD-plasticity in vivo. The reason that accounts for this may be linked to a complex cascade of pathophysiological events and changes in the metabolic status of the cerebral cortex that happen shortly after stroke and make the inhibition of plasticity likely. After a stroke a complex series of pathophysiological events develop and evolve in time and space (Dirnagl et al. 1999). Immediate pathologies, such as blood supply restrictions, energy failure, decrease in ion pump activity, excitotoxicity (Choi 1992), peri-infarct depolarizations (Dietrich et al. 1994), inflammation (Schroeter et al. 2002; Wang et al. 2007), programmed cell death (apoptosis) and edema (Dirnagl et al. 1999; Schaller \& Graf 2004), are unlikely to support plastic changes (Figure 79). 


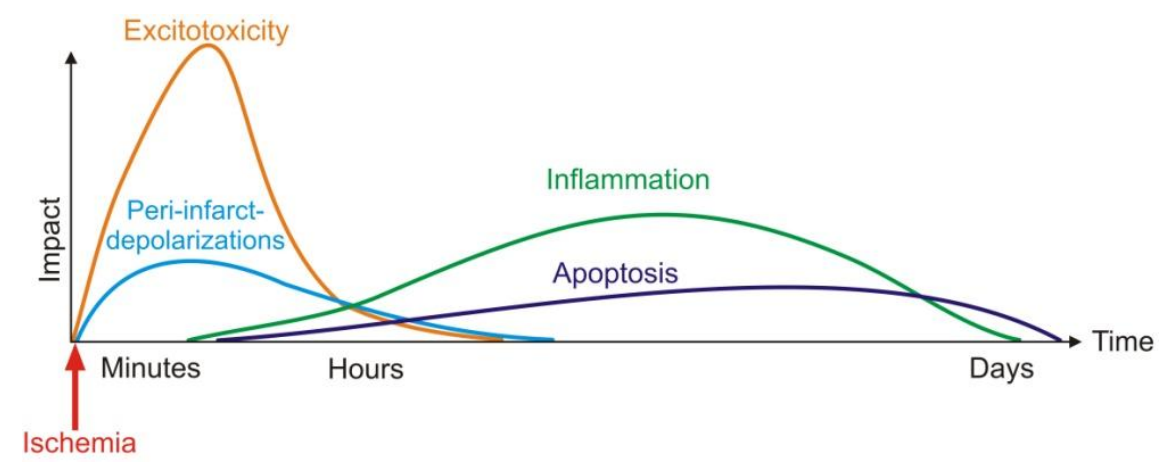

Figure 79: Putative cascade of damaging events after cerebral ischemia. Very early after the ischemia, excitotoxic mechanisms can damage neurons and glia lethally. In addition, excitotoxicity triggers a number of events that can further contribute to the damage of the tissue. Such events include peri-infarct depolarizations and the more-delayed mechanisms of inflammation and programmed cell death. The $\mathrm{x}$-axis reflects the development of the cascade over time, and the y-axis the impact of each event of the cascade on final outcome. Modified from Dirnagl et al. (1999).

Several in vitro studies in rats indicated that plasticity mechanisms are actually increased in the surround of an ischemic lesion (about $0.5-3 \mathrm{~mm}$ away from the lesion): GABAergic inhibition is decreased (Neumann-Haefelin et al. 1995; Qü et al. 1998), N-methyl-D-aspartate (NMDA) receptor binding is increased (Que et al. 1999), as well as long-term potentiation (LTP) is increased (Hagemann et al. 1998; Mittmann \& Eysel 2001). However, these in vitro experiments may have only limited validity for complex changes taking place in nerve cell networks in vivo. LTP and its counterpart long-term depression (LTD) are synaptic processes at nerve cells, which are the synaptic basis of learning, memory and neuronal plasticity in vertebrates (Bliss \& Gardner-Medwin 1973; Bliss \& Lomo 1973; Doyere \& Laroche 1992; Bliss \& Collingridge 1993). LTP is a long lasting enhancement in signal transmission due to a repeated and synchronous input between neurons, whereas LTD results in a reduction in signal transmission. Mittmann \& Eysel (2001) found a disturbed balance of LTP and LTD in the rat visual cortex in the first week after an infrared-laser lesion. LTP and LTD are essential mechanisms for the strengthening and weakening of synaptic connections. OD-plasticity after MD is mediated by weakening connections of the deprived eye and enhancing connections of the open eye (Antonini \& Stryker 1993; Frenkel \& Bear 2004). A disturbed balance between LTP and LTD could consequently impair coordinated synaptic strengthening and weakening and affect neuronal plasticity. Protein synthesis is a necessary component of the late phase of LTP to establish synaptic modifications - the basis of cortical plasticity. After stroke though, protein synthesis is impaired for several days (Hata et al. 2000). Peri-lesional areas may show enhanced activation (Hagemann et al. 1998; Mittmann \& Eysel 2001) and decreased inhibition (Neumann-Haefelin et al. 1995; Qü et al. 1998) in electrophysiological 
experiments, but this may not be translated into long-lasting synaptic modifications, because of disturbed protein synthesis.

Concluding, the in vitro results (increased plasticity mechanisms in the vicinity of a lesion) and in vivo results (diminished plasticity) are not necessarily contradictory but rather describe different levels of complexity and network interactions.

A methodological consideration. Signals from the intrinsic optical imaging are mainly based on the change in reflectance of circulating blood due to higher light absorption of deoxyhemoglobin, which briefly accumulates in active brain tissue due to the increased oxygen consumption of active neurons (Pouratian \& Toga 2002). Does the PT-lesion affect the blood circulation in the visual area and thereby the optical imaging signal and the measured OD-plasticity? To answer this question, additional electrophysiological experiments, which do not rely on changes in the blood flow, were performed. The result confirmed the data obtained by optical imaging of intrinsic signals: there was no ODplasticity in the surround of a PT-lesion. In addition, there is more evidence that the PT-lesion is unlikely to affect the optical imaging measurements by disturbing the blood flow. The PTmodel used in our experiments induces a well-described lesion without a widespread penumbra-like region of reduced blood flow (Hossmann 2008). Zhang \& Murphy (2007) revealed that outside of a small $400 \mu \mathrm{m}$ rim around the PT-lesion, the blood flow is unimpaired and cortical hemodynamic responses in response to sensory stimuli are normal. Furthermore, for the quantification of OD-plasticity based on intrinsic signal optical imaging, we always compared the relative cortical activation levels after visual stimulation of the ipsiand contralateral eye in the identical part of V1. This relative measure should be independent of absolute activity levels and not modified by blood flow changes.

It is therefore unlikely that our lesion considerably changed the blood flow in the visual cortex and affected the OD-plasticity measurements by optical imaging.

\subsection{Networks and two hemispheres}

Why is OD-plasticity impaired although the visual cortex is not directly lesioned? The idea that lesions can cause also remote, even transhemispheric, effects and transient alterations of brain functions came up in the $20^{\text {th }}$ century and this effect has been termed "diaschisis" by Von Monakow (Von Monakow 1914; Andrews 1991; Finger et al. 2004). Von Monakow wanted to discriminate between the deficits observed in patients that result directly from a 
focal brain lesion and the loss of function in a brain region o that is not directly damaged but neurally connected to a distant lesioned brain area. According to our data such non-local impact might be also important for OD-plasticity, more than previously thought. A lesion outside V1 affected its OD-plasticity. Thus, OD-plasticity is likely to be co-determined by network influences from outside V1.

S1 - V1 connection. One example of such a network influence is the S1 - V1 connection. A monosynaptic anatomical connection between S1 and V1 could be shown in tracing studies (Di Garbo et al. 2011). Di Garbo et al. (2011) injected a retrograde neuronal tracer into V1 of adult (PD 60) C57BL/6J mice and found labeled cell bodies in S1. A photostimulation (with channelrhodopsin-2, ChR2) of the hindlimb area of S1 (were our PT-lesion was primarily located) also caused an activation of V1 in C57BL/6J mice (Lim et al. 2012). Several studies indicate also functional cross-modal influences between the somatosensory and visual cortex in humans as well as in rodents. For instance, in humans that were blind from an early age on, V1 could be activated by somatosensory input, like by Braille reading and other tactile discrimination tasks (Sadato et al. 1996; Cohen et al. 1997). Moreover, tactile performance in normally sighted, blindfolded subjects also increased activation in V1 (Merabet et al. 2007). In rodents V1 responses were reported in rats after somatosensory stimulation (Wallace et al. 2004; Frostig et al. 2008; Vasconcelos et al. 2011). These results show that V1 gets functional input from other cortical areas, like the somatosensory area, where the PT-lesion in our studies was located. Lesioning S1 would disrupt its input to V1 and might therefore influence neuronal network interactions in V1. To investigate how the lesion affects the input from S1 to V1, subsequent optogenetic stimulation experiments could be performed. Specific stimulation of $S 1$ neurons could be achieved by injecting a virus with the light-gated cation membrane channel $\mathrm{ChR} 2$ virus into $\mathrm{S} 1$. Once $\mathrm{ChR} 2$ is expressed it can be activated with blue light $(\sim 470 \mathrm{~nm})$ : the channel opens and allows entry of cations, mostly $\mathrm{Na}^{+}$, into the cell and thereby drives action potential firing (Nagel et al. 2003; Boyden et al. 2005; for reviews see for example Nagel 2005; Zhang et al. 2007). While activating the ChR2 neurons in S1, the evoked activity of neurons that connect to V1 might possibly be measured with optical imaging of intrinsic signals (Lim et al. 2012; Scott \& Murphy 2012). Using the ChR2 stimulation alone or in combination with visual stimuli, as used in our experiments, in nonlesioned mice, can give us an idea how input from the lesioned area can modify signals in V1 and if the lacking S1 input after PT might account for the diminished OD-plasticity.

Neuromodulatory systems. Another possible explanation for the influence of a cortical lesion in $\mathrm{S} 1$ on OD-plasticity in V1 could be disturbances in the neuromodulatory systems. The 
main neuromodulators in the brain are acetylcholine, noradrenalin, serotonin (5hydroxytryptamin, 5-HT) and dopamine. The cell bodies of neuromodulatory neurons are grouped in specific nuclei in the forebrain and midbrain from where their widespread projections influence many brain regions. There the projections run to V1 through the cortex and possibly passing by the lesion site (for projections see - cholinergic: Luiten et al. (1987) (Figure 80), serotonergic: Waterhouse et al. (1986) Vertes (1991), noradrenergic: Eschenko et al. (2012)). For example in the cholinergic system, a tracing study by Luiten et al. (1987) in rats showed, that fibers project from the nucleus basalis through the somatosensory cortex to the visual cortex (Figure 80). The PT-lesion in the somatosensory cortex might disrupt the projections to V1 and therefore the normal supply of the neuromodulators, which might explain the diminished OD-plasticity in V1. 

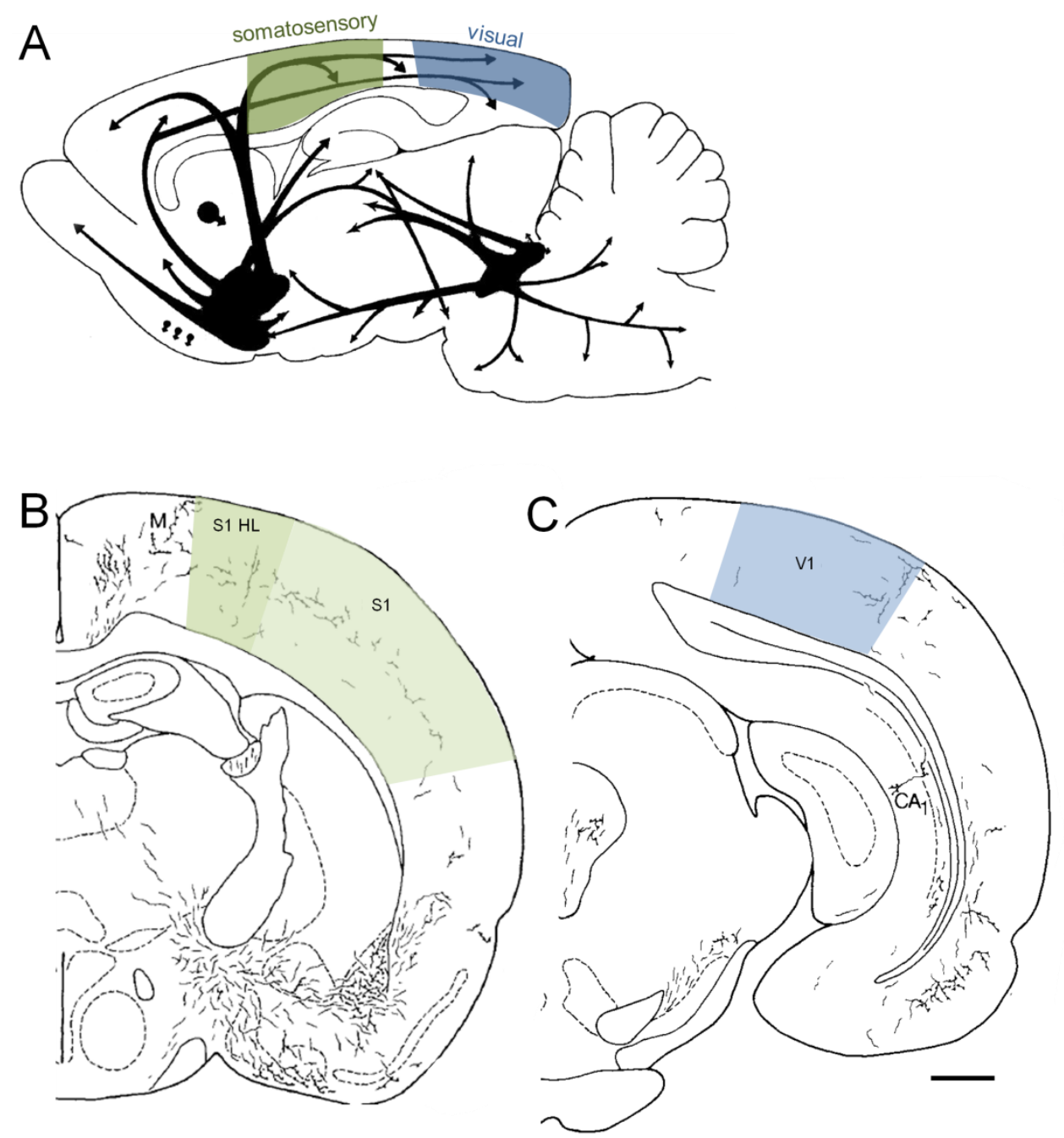

Figure 80: Cholinergic projection trough the somatosensory cortex to the visual cortex. (A) Scheme of the central projections of cholinergic cells shown in a sagittal section from rat brain. The cortex is innervated by the basal forebrain subsystem with fibers projecting from anterior through the somatosensory area to the posteriorly located visual cortex. (B \& C) Coronal sections in an anterior to posterior sequence showing cholinergic fibers labeled with an anterograd tracer (injection site in basal nucleus: anterior part of the magnocellular basal nucleus) overlaid with cortex regions (according to Paxinos rat brain atlas (Paxinos \& Watson 2005)) in a rat. Projections run from the basal nucleus, through S1, including the hindlimb region (HL) (A), where typically the PT-lesion is located in our animals, to V1 (B). (A) Modified from Woolf (1991). (B \&C) Modified from Luiten et al. (1987), scale bar: $1 \mathrm{~mm}$.

It has been shown that modulation of these neuromodulatory systems influences OD-plasticity (Bear \& Singer 1986; Gu \& Singer 1995; Maya Vetencourt et al. 2008; Morishita et al. 2010). Disrupting the cholinergic, noradrenergic and/or serotonergic transmission impeded ODplasticity (Bear \& Singer 1986; Gu \& Singer 1995). In turn, enhancing neuromodulatory systems can increase OD-plasticity as shown in rodents for the cholinergic (Morishita et al. 2010) and serotonergic system (Maya Vetencourt et al. 2008). In order to supplement for the potential deficit of acetylcholine, PT-mice could be treated with an intraperitoneal injection of 
the acetylcholinesterase inhibitor physostigmine to increase acetylcholine levels (Morishita et al. 2010). If the deficit of acetylcholine was responsible for the diminished OD-plasticity, it should be restored by that treatment. To test whether an increase in noradrenalin levels could reestablish OD-plasticity after stroke, mice could be treated with noradrenalin reuptake inhibitors like reboxetine (Weber et al. 2009) or desipramine, which has been already used in mice (e.g. Lucas et al. (2012)). If a lack of serotonin is responsible for the diminished ODplasticity in PT-mice, treatment with the serotonin reuptake inhibitor fluoxetine should restore OD-plasticity. In fact, it has been shown that serotonin plays an important role in rehabilitation after stroke. Experimental studies have demonstrated beneficial effects of 5$\mathrm{HT}_{1 \mathrm{~A}}$ receptor agonists after ischemia in rats, like reduced infarct size and neuronal damage (Prehn et al. 1991; Prehn et al. 1993; Semkova et al. 1998; Torup et al. 2000). Moreover, a recent clinical trial (the "fluoxetine for motor recovery after acute ischemic stroke (FLAME) trial"), demonstrated that a 3 months treatment with the serotonin reuptake inhibitor fluoxetine, starting 5 to 10 days after the onset of stroke, led to an enhanced motor recovery in the stroke patients (Chollet et al. 2011).

Further experiments in which the neuromodulators are supplemented can provide further insights for their role in diminished OD-plasticity after a PT-lesion and might reveal how ODplasticity after a PT stroke lesion could be restored.

Interhemispheric inhibition. Besides possible intracortical influences between brain areas within one hemisphere, as just described, there are also influences from the other hemisphere. These long-ranging intercortical interactions are mediated through the corpus callosum. Such an interaction is the intercortical inhibition: both hemispheres inhibit each other through the corpus callosum (Hummel \& Cohen 2006). A lesion, like a stroke, in one hemisphere might disrupt this interaction, causing a decreased inhibition and increased excitation in the nonlesioned hemisphere (Buchkremer-Ratzmann et al. 1996; Liepert et al. 2000; Manganotti et al. 2002; Shimizu et al. 2002; Cicinelli et al. 2003). Indeed, clinical functional magnetic resonance imaging studies found an increased activation of the non-lesioned hemisphere partly even up to years after the stroke (Carey et al. 2002; Gerloff et al. 2006). Accordingly, the lesioned hemisphere will be even more inhibited by the non-lesioned hemisphere (Murase et al. 2004). Enhanced activity supports synaptic plasticity and learning processes (Artola \& Singer 1987), whereas reduced activity might prevent experience-dependent changes in neuronal circuitry. Thus, our observation of absent OD-plasticity in the lesioned hemisphere and present (even enhanced) OD-plasticity in the non-lesioned hemisphere, might be explained by reduced activity in the lesioned hemisphere and increased activity in the non- 
lesioned hemisphere. In fact, in PT-mice we observed weaker maximal response strength in the lesioned hemisphere compared to the non-lesioned hemisphere. Clinical studies also demonstrate that increased activation of the non-lesioned hemisphere can hinder the regeneration of the lesioned hemisphere (Liepert et al. 2000; Ward et al. 2003; Ward 2004; review: Hummel \& Cohen 2006). Downregulating excitability in the non-lesioned hemisphere (Mansur et al. 2005; Takeuchi et al. 2005) or upregulating excitability in the lesioned hemisphere (Hummel et al. 2005; Khedr et al. 2005) in fact resulted in improved motor function in patients with stroke (for a review see Hummel \& Gerloff 2012; Schulz et al. 2013). Consequently, diminished plasticity in the lesioned, and present or even enhanced plasticity in the non-lesioned hemisphere of PT-animals might be at least partly due to changes in neuronal activity levels. Recently, Failor et al. (2010) also suggested, that ODplasticity depends crucially on the precise regulation of cortical activation: In rats with moderate cortical injury following neonatal hypoxia-ischemia, impaired OD-plasticity was correlated with a modified excitation-inhibition balance. If the reduced activity in the lesioned hemisphere is causal for the diminished OD-plasticity, than there should be a correlation between the hemisphere activation quotient (activation the non-lesioned hemisphere divided by activity of the lesioned hemisphere) and their ODI after MD in PT-mice. However, we could not find such a correlation.

Hence, the difference in the activation levels of the lesioned and non-lesioned hemisphere cannot entirely account for the diminished plasticity after stroke.

Callosal connections. Callosal connections between the two hemispheres have been also shown to be crucial for OD-plasticity in rats (Restani et al. 2009). A substantial portion of the influence of the ipsilateral eye on cortical responses arrived via callosal connections from the opposite hemisphere, and these afferents played an important role in the reduction of inputs from the deprived eye during MD. When looking at OD-plasticity in the lesioned and nonlesioned hemisphere in our PT-mice, callosal influences seem also likely. In the lesioned hemisphere OD-plasticity was absent in PT-mice after MD of the contralateral eye. However, if the ipsilateral eye was deprived, OD-plasticity could be still induced. In the non-lesioned hemisphere of PT-mice after contralateral MD, OD-plasticity was present, although less pronounced than in control mice. After ipsilateral MD, OD-plasticity was also present. Thus, the effect of the PT-lesion on OD-plasticity is specific, dependent whether the contra- or ipsilateral eye was deprived. 
Lesioned hemisphere. In our PT-animals, callosal inputs from the non-lesioned hemisphere that contribute to ipsilateral eye input should have remained intact, but nevertheless ODplasticity was absent after contralateral eye MD. This may be due to disruptions in the S1 V1 connection or neuromodulatory systems as described in the paragraph above. However, OD-plasticity in the lesioned hemisphere was not completely abolished, because it was still inducible after ipsilateral eye MD. How can that be explained? In mice, neurons in the binocular visual cortex are typically dominated by contralateral input (Dräger 1975; Mangini \& Pearlman 1980; Wagor et al. 1980; Metin et al. 1988). This direct stronger contralateral input, which should not be affected by the PT-lesion, might be enough to induce ODplasticity after ipsilateral MD, despite the disruptions in the $\mathrm{S} 1$ - V1 connection or neuromodulatory systems.

Non-lesioned hemisphere. OD-plasticity in the non-lesioned hemisphere could be induced by contralateral MD, but was less pronounced than typically observed in control mice. The findings of Restani et al. (2009) might provide an explanation for that phenomenon as well. They described that an essential portion of the ipsilateral eye influences arrives via callosal connections from the opposite hemisphere, and that these afferents are important for the reduction of deprived eye inputs during MD. In our case, the opposite hemisphere is lesioned and therefore the transmission of the ipsilateral eye input to the non-lesioned hemisphere via the lesioned hemisphere and the corpus callosum might be disturbed. This means that the reduction of the deprived eye responses, mediated via this route, might be smaller. This leads to a reduced OD-shift. Further experiments provide evidence for that theory: blocking activity in V1 of one hemisphere during MD reduced the OD-shift (Restani et al. 2009). Now, why can we observe OD-plasticity in the non-lesioned hemisphere after ipsilateral eye MD, although input from the lesioned hemisphere is impaired? Even though input from the deprived ipsilateral eye is mediated via the corpus callosum, the more important and dominant input comes directly from the contralateral eye. This connection should not be affected by the lesion and therefore OD-plasticity is still possible.

As a result, our data clearly indicate that OD-plasticity is not just a local phenomenon but rather co-determined by intracortical and callosal intercortical network influences from outside V1.

\section{Inhibition and excitation}

Another mechanism that might be involved in the diminished OD-plasticity after PT is the inhibition/excitation balance. Neuronal circuits in the cortex consist primarily of 
glutamatergic neurons which are excitatory and GABAergic neurons which are inhibitory (for a review see McCormick 1992; Moore 1993). A recent study revealed that these two types of neurons contribute differently to OD-plasticity in adult mice (Kameyama et al. 2010). The main cellular mechanism underlying OD-plasticity in adult mice is a change in the response of GABAergic inhibitory neurons. After MD, responses of inhibitory GABAergic neurons to the deprived eye were reduced, while open-eye responses of excitatory neurons were only slightly potentiated (Kameyama et al. 2010). The lacking OD-plasticity in our PT-animals might therefore be due to a - by a so far unknown mechanism - weaker depression of responses of GABAergic neurons to deprived eye stimulation which in turn could cause stronger inhibition and obstruct plastic changes in V1. The observed smaller V1 activation in the lesioned hemisphere after visual stimulation found in our PT-animals also supports this hypothesis. Further, this is consistent with the observation that intracortical inhibition is an essential limiting factor for experience-dependent plasticity in the adult visual cortex (Harauzov et al. 2010).

To sum up, the diminished OD-plasticity after a PT-lesion might be due to changes in the GABAergic inhibition.

\subsection{Enriched environment boosts OD-plasticity}

EE is known to promote plasticity mechanisms (Sale et al. 2004; Sale et al. 2007; Baroncelli et al. 2010b). The effect of EE-housing of 2 to 3 weeks particularly on OD-plasticity has been studied in rats (Sale et al. 2007; Baroncelli et al. 2010b; Baroncelli et al. 2012; Scali et al. 2012). However, our studies for the first time analyzed how EE-housing from before birth and EE-housing later in life influences OD-plasticity in adult mice. It was quite striking that a very pronounced OD-shift could be still induced in old mice, when they were raised in EE (oldest mouse was already 196 days old). Whereas in SC-mice no OD-shift was inducible any more after PD 110 in the optical imaging experiments (Lehmann \& Löwel 2008). The ODplasticity in our EE-mice was so strong that the OD-shift was as prominent as in PD 30 mice in the critical period, where typically the OD-shift is most pronounced. The OD-shift found by Scali et al. (2012) and Baroncelli et al. (2010b) after EE-housing of rats, was not as prominent as in SC-rats during the critical period (Caleo et al. 1999; Mandolesi et al. 2005; Restani et al. 2009; Spolidoro et al. 2012). This is most probably due to the shorter time of EE-housing they used, and the later onset. Rats were housed in EE for just 3 weeks when they were already adult. Our mice were housed in EE from before birth for at least 4 months. 
Moreover, the OD-shift we observed in adult mice raised in EE was juvenile-like: the shift was mediated by a decrease in the cortical responses after deprived eye stimulation as described for juvenile SC-mice in the critical period (Hofer et al. 2006b; Heimel et al. 2007). In contrast to that, an "adult" OD-shift is usually mediated by an increase in the cortical responses after open eye stimulation (Sawtell et al. 2003; Hofer et al. 2006b; Mrsic-Flogel et al. 2007; Sato \& Stryker 2008). Consequently, our results show that EE can prolong the sensitive phase for OD-plasticity into late adulthood, and that this OD-plasticity is even juvenile-like.

Since this can potentially open a non-pharmacological route for the enhancement of cortical plasticity, we tested in addition whether EE-housing can also restore OD-plasticity in adult mice raised in a SC. Indeed, in mice transferred to EE-housing at PD 110, an age where ODplasticity was not inducible any more in SC-mice (Lehmann \& Löwel 2008), OD-plasticity could still be induced up to an age of 320 days. However, the OD-shift was less pronounced compared to mice raised in EE. Moreover, it was not clear whether the shift represents a juvenile or adult form of OD-plasticity: neither the decrease of deprived eye responses, nor the increase of open eye responses was significant. Summarizing, EE has the potential to enhance cortical plasticity in the adult brain and EE-housing from birth was more efficient in maintaining an enhanced OD-plasticity.

Interestingly, in the optical imaging experiments the response magnitude in V1 after visual stimulation was lower in EE-mice than in SC-mice. This was also true for mice with a PTlesion. A recent study might explain this phenomenon. Di Garbo et al. (2011) recorded simultaneously the activity (local field potentials) in V1 and in the secondary motor cortex in adult (PD 60), awake, freely-moving mice (C57BL/6J) to quantify the synchronization level of the two cortical areas. Mice that were reared in EE, showed decreased levels of coupling of the activity in the two cortical regions (Di Garbo et al. 2011). This work is in good agreement with our data since after EE-rearing we observed a decreased activity in V1 in the optical imaging experiments. A decreased coupling of V1 with other cortical areas such as the motor cortex after EE-rearing, might explain a decreased activity of neurons in V1, because additional activation of V1 through other cortical areas is lacking.

There are still open questions about the effect of EE-housing on OD-plasticity that should be addressed in future experiments. All mice raised in EE showed OD-plasticity up to PD 196. One question is: how long will the mice stay plastic, or will they even show OD-plasticity their whole life long? Moreover, could this OD-plasticity still be juvenile-like? Will the EE- 
mice live even longer due to the beneficial effects of EE? Given that EE-mice showed ODplasticity similar to the critical period still in late adulthood, the question is whether the critical period in EE-mice is shifted and starts later in life (and persists longer) or whether critical period opens and just never closes.

\subsection{Are inhibitory circuits modified after EE in mice?}

What might be the mechanism underlying the prolonged OD-plasticity in EE-mice? It has been shown, that restored OD-plasticity after EE-housing in adult rats was casually linked to reduced extracellular levels of the inhibitory neurotransmitter GABA (Sale et al. 2007; Baroncelli et al. 2010b). When inhibition in these EE-rats was increased by applying diazepam, a positive allosteric $\mathrm{GABA}_{\mathrm{A}}$ receptor modulator, the restored OD-plasticity was completely prevented (Sale et al. 2007; Baroncelli et al. 2010b). To test whether an increased GABAergic inhibition might block the prolonged OD-plasticity in our EE-experiments as well, we applied diazepam during the MD-period, with a dosage that reliably blocked the ODshift in SC-mice. Unlike the other two studies, where OD-plasticity was completely blocked by diazepam treatment, we just observed partly abolished OD-plasticity. Thus, in our experiments reduced GABAergic inhibition can just partially account for the prolonged ODplasticity after EE-rearing, suggesting involvement of additional mechanisms. The diverging results are most likely due to the timing of EE-housing. In the studies of Sale et al. (2007) and Baroncelli et al. (2010b) EE exposure lasted just 2 to 3 weeks, starting when the rats were already adult. In our study mice were at least 5 months housed in the EE from before birth. Therefore mechanisms elicited by EE might be more consolidated and less susceptible to increased inhibition in our experimental design. Although the used dosage in our studies was capable of blocking the OD-shift in SC-mice, EE-housing might change the susceptibility to diazepam so that the applied dosage of diazepam would be too low to increase GABAergic inhibition enough and to affectively block the OD-shift. Consequently, the effect of diazepam might be reduced in our EE-mice and it is possible that a higher dosage of diazepam would have blocked the OD-shift completely, like in SC-mice. Taking into account that EE-housing lowers extracellular GABA levels in V1 of rats (Sale et al. 2007; Baroncelli et al. 2010b), a higher dosage of diazepam or a more local application (for example via minipump into the visual cortex) might be necessary to compensate for reduced GABA levels, to reach the same level of GABAergic inhibition as in SC-mice. Likewise, Simpson et al. (2012) observed that rats housed in EE showed reduced sensitivity to the behavioral effects of diazepam. They used 
diazepam as an anxiolytic drug (Valium) and noticed that EE-housing attenuated diazepam effects on their anxious behavior in the elevated plus maze.

In addition to the optical imaging experiments, patch-clamp experiments were performed in SC- and EE-mice in cooperation with the group of Dr. Dr. Oliver Schlüter (ENI, Göttingen) by the PhD student Plinio Favaro. Whole-cell patch-clamp recordings were carried out in the visual cortex in coronal brain slices $(400 \mu \mathrm{m})$. A concentric bipolar stimulating electrode was placed at the region of layer IV and recording were done from layer II/III pyramidal cells. EErearing did not affect the basal excitatory synaptic transmission in the layer IV to II/III pathway in the visual cortex: the ratio of the AMPA ( $\alpha$-amino-3-hydroxy-5-methyl-4isoxazolepropionic acid) receptor mediated excitatory postsynaptic currents (EPSCs) and NMDA receptor mediated EPSCs was not significantly different between SC- and EE-mice (>PD 130). Nonetheless, these experiments revealed an altered inhibition/excitation balance after EE-rearing: the ratio of GABA receptor mediated inhibitory postsynaptic currents (IPSCs) and AMPA receptor mediated EPSCs were significantly different between SC- and EE-animals (>PD 130). The change in the ration was mainly due to a reduction in GABA receptor mediated responses.

To further address the point of altered inhibition after EE-rearing we quantified PV-positive inhibitory interneurons in V1. The number of PV-positive cells was not different between SCand EE-mice. We analyzed particularly PV-positive interneurons, because these GABAergic interneurons are thought to play a crucial role in OD-plasticity (reviewed in Espinosa \& Stryker 2012). However, PV-positive neurons account just for $39 \%$ of GABAergic inhibitory interneurons in the mouse visual cortex (Gonchar et al. 2007). Thus, other GABAergic interneurons, like calretinin or and somatostatin could still mediate a change in GABAergic inhibition. Moreover, even if the number of PV-positive cells is not changed, the concentration of PV within the cells might be different in EE-mice and can in turn affect inhibition. For example a higher concentration of the calcium binding protein PV in the inhibitory interneurons in EE-mice might act as a stronger calcium buffer, which can consequently reduce GABA-vesicle release and therefore inhibition (Eggermann \& Jonas 2012). In a subsequent experiment we could analyze whether the PV-concentration is changed within the cell by determining the fluorescence intensity of the PV-stained cells.

Taken together, these results suggest that a reduction in the inhibitory tone might be involved in the prolonged OD-plasticity found in EE-mice. 


\subsection{What other possible mechanisms are there?}

What other mechanisms might account for the prolonged OD-plasticity after raising mice in $\mathrm{EE}$ ?

PNNS

The degradation of PNNs has been shown to play a role in the reopening of OD-plasticity in adult rats (Pizzorusso et al. 2002; Harauzov et al. 2010). Sale et al. (2007) as well as Scali et al. (2012) found reduced PNN density (using WFA (Wisteria floribunda agglutinin) staining) in the visual cortex after EE-housing in rats, which also showed restored OD-plasticity. We also analyzed the number of WFA-stained PNNs in V1 of mice. In contrast to the other two studies we did not observe any difference between SC- and EE-mice. The different results might be due to the experimental designs of the studies. Sale et al. (2007) as well as Scali et al. (2012) housed rats in EE just for a short time (2 to 3 weeks) when the animals were already adult (PD 60 and 22-24 month, respectively). In our study, mice were born in the EE and spend at least 5 months in this housing condition. Therefore the PNN density in our EEmice might have developed normal levels (like in SC-mice) through the time (Bartoletti et al. 2004) and since in the other two studies the EE-housing was a rather sudden stimulus, PNN density might have been reduced in response to that.

\section{Serotonin}

It has been shown that an increase in serotonin levels can restore OD-plasticity in adult rats (Maya Vetencourt et al. 2008; Maya Vetencourt et al. 2011). After EE-housing, Baroncelli et al. (2010b) found increased serotonin levels in V1 of adult rats, together with a restored ODplasticity. When they reduced the serotonergic transmission in EE-rats, the restored ODplasticity was completely abolished. EE-housing can also enhance expression of the gene for the serotonin 1A $\left(5-\mathrm{HT}_{1 \mathrm{~A}}\right)$ receptor in the hippocampus of rats (Rasmuson et al. 1998). Studies in rats suggest a key role of the $5-\mathrm{HT}_{1 \mathrm{~A}}$ receptor for the reactivation of OD-plasticity in adult rats (Maya Vetencourt et al. 2011). According to the Allen Mouse Brain Atlas (Lein et al. 2007), expression of 5-HT $1 \mathrm{~A}$ can be also found in the visual cortex of C57BL/6J mice (Allen Institute for Brain Science 2012). Thus, it is quite possible that the 5- $\mathrm{HT}_{1 \mathrm{~A}}$ receptor is also crucially involved in the OD-plasticity in mice. Consequently, the prolonged ODplasticity in our experiments might be due to increased serotonin levels or an increased expression of serotonin receptor in the visual cortex. In order to test this, one could collect homogenates of $\mathrm{V} 1$ of mice raised in $\mathrm{EE}$ and determine serotonin levels by means of high 
performance liquid chromatography analysis as, described by Atkinson et al. (2006) and Baroncelli et al. (2010b), and compare them to SC-mice. Moreover, to find out whether an increase of serotonin levels is causally linked to the prolongation of OD-plasticity, serotonergic transmission in EE-mice could be reduced by para-chlorophenylalanine during the time of MD. This substance can be applied via an osmotic minipump in the visual cortex as in the study of Baroncelli et al. (2010b). A less invasive application for mice was also described via intraperitoneal injection (Estler 1973; Dailly et al. 2006).

As mentioned, one of the main cellular mechanism underlying OD-plasticity in adult mice is a change in responses of inhibitory GABAergic neurons, while responses of excitatory neurons were only slightly modified (Kameyama et al. 2010). These measurements were performed in the superficial layers of the mouse visual cortex where OD-plasticity in mice beyond the critical period mostly occurs (Pham et al. 2004; Tagawa et al. 2005). However, the largest group of GABAergic interneurons in the superficial layers has not been studied yet: interneurons that express the serotonin 5- $\mathrm{HT}_{3 \mathrm{~A}}$ receptor (Lee et al. 2010). According to Lee et al. (2010) the 5-HT $3 \mathrm{~A}$ interneurons are the largest population of interneurons (about $60 \%$ of all interneurons) in the superficial layers (I-III) of the somatosensory cortex of mice. Activation of the 5- $\mathrm{HT}_{3 \mathrm{~A}}$ receptor channel results in an enhancement of GABAergic synaptic transmission (Lee et al. 2010). Moreover, some 5- $\mathrm{HT}_{3 \mathrm{~A}}$ expressing neurons receive weak but direct monosynaptic input from the thalamus (Lee et al. 2010). The Allen mouse brain atlas shows an expression of $5-\mathrm{HT}_{3 \mathrm{~A}}$ also in the visual cortex of mice (Allen Institute for Brain Science 2012). The role of the 5- $\mathrm{HT}_{3 \mathrm{~A}}$ receptor in OD-plasticity of mice is not clear yet. Nonetheless, the mentioned observations and the fact, that serotonin is involved in ODplasticity of the adult visual cortex (Maya Vetencourt et al. 2008), suggests a potential role of $5-\mathrm{HT}_{3 \mathrm{~A}}$ receptor positive interneurons in the prolonged OD-plasticity after EE-housing. In order to compare expression of the 5- $\mathrm{HT}_{3 \mathrm{~A}}$ receptors in $\mathrm{V} 1$ of $\mathrm{SC}$ - and EE-mice, a transgenic mouse line $\left(5-\mathrm{HT}_{3 \mathrm{~A}} \mathrm{R}-\mathrm{BAC}{ }^{\mathrm{EGFP}}\right)$ in which $5-\mathrm{HT}_{3 \mathrm{~A}}$ positive interneurons are fluorescently labeled could be used. Moreover, there are antibodies for 5- $\mathrm{HT}_{3 \mathrm{~A}}$ receptor commercially available that react with mouse tissue (e.g. Abcam antibody ab13897) and can be used for immunohistochemical analysis of 5- $\mathrm{HT}_{3 \mathrm{~A}}$ receptor expression. To test whether a lack of 5$\mathrm{HT}_{3 \mathrm{~A}}$ receptors would affect OD-plasticity in SC- and EE-mice, a 5-HT $\mathrm{H}_{3 \mathrm{~A}}$ knockout mouse could be used, which was initially designed by Zeitz et al. (2002).

Concluding, it would be interesting to further test experimentally the potential involvement of the serotonergic neuromodulatory system in the prolonged OD-plasticity after EE-housing. 
$B D N F$

Another crucial factor for the enhanced OD-plasticity after EE-housing might be the brainderived neurotrophic factor (BDNF). Baroncelli et al. (2010b) and Sale et al. (2007) showed that restored OD-plasticity in EE-rats was also accompanied by an increased BDNF expression in the visual cortex of rats. In mice reared from birth in EE, precociously increased BDNF levels (at PD 7) in the visual cortex were also reported (Cancedda et al. 2004). In adult SC-rats, intracortical administration of BDNF could reactivate OD-plasticity in adult rats (Maya Vetencourt et al. 2008). Conclusively, the prolonged and restored OD-plasticity in our mice could be mediated by increased BDNF levels. To determine whether BDNF levels are increased in our EE-mice compared to SC-mice an enzyme-linked immunosorbent assay (ELISA) could be used (Ickes et al. 2000; Cancedda et al. 2004; Sale et al. 2004). In order to assess whether the rise of cortical BDNF has a critical role in the prolongation of ODplasticity in our EE-mice, BDNF levels could be reduced in EE-mice during MD. To do so, an intracortical infusion of BDNF antisense oligonucleotides, which blocks the expression of BDNF, could be applied in EE-mice via osmotic minipumps as previously reported in the rat central nervous system (Ma et al. 1998; Landi et al. 2007; Ploughman et al. 2009; Baroncelli et al. 2010b). If there is a causal link, prolonged OD-plasticity in EE-mice should be blocked by that treatment.

\section{Histone acetylation}

Environmental changes can also alter the brain chromatin status (reviewed in Sweatt 2009). EE-housing led to increased levels of histone acetylation in the hippocampus and cortex, which has been implicated in transcriptional regulation of gene expression via chromatin modification (Fischer et al. 2007). Such epigenetic modifications have been recently shown to play a role in the regulation of OD-plasticity in the adult visual cortex. Visual experience activates histone acetylation in the visual cortex during the critical period, but this capacity is downregulated in adult animals (Putignano et al. 2007). Pharmacological treatment that promoted histone acetylation and therefore translation, enhanced OD-plasticity in adult mice (Putignano et al. 2007). Consequently, prolonged OD-plasticity in our experiments might be mediated through increased levels of histone acetylation. To test this hypothesis, the level of histone acetylation could be measured in visual cortex lysates probed with antibodies detecting histone-tail modification using quantitative western blot analysis in EE-mice and could be compared to SC-mice. Histone deacetylases (HDACs) are enzymes that alter the chromatin structure locally by removing acetyl groups from histone core proteins and thereby 
causing a tighter wrapping of DNA around the histones (for a review see Fagiolini 2009). This compaction of chromatin leads to transcriptional suppression. If the prolonged ODplasticity in EE-mice is in fact mediated via an increased histone acetylation, an increasing histone acetylation in SC-mice of the same age by inhibiting histone deacetylases should also facilitate OD-plasticity. Frequently used inhibitors of histone deacetylases are sodium butyrate and trichostatin A (Fischer et al. 2007; Putignano et al. 2007; Silingardi et al. 2010) and suberoylanilide hydroxamic acid (SAHA; also known as Vorinostat or Zolinza). SAHA is especially interesting as a drug which might facilitate plasticity, because it has been already approved for human application in skin cancer treatment (Marks \& Breslow 2007).

Conclusively, these studies show that serotonin, BDNF and histone acetylation are crucial factors in EE mediated effects on plasticity and that they might account for the prolonged and restored OD-plasticity we found.

\subsection{How can EE preserve OD-plasticity after stroke?}

Raising mice in EE preserved OD-plasticity and partially sensory learning after a PT stroke. What effect of EE might account for that preserved plasticity?

\section{Inflammation}

Ruscher et al. (2009) found that EE-housing after a middle cerebral artery occlusion (MCAO) in rats reduced the increased level of the pro-inflammatory cytokine interleukin-1 $\beta$. Another recent study showed that neutralization of interleukin- $1 \beta$ reduced tissue loss and improved late cognitive outcome after a traumatic brain injury in mice (Clausen et al. 2011). We observed that anti-inflammatory treatment with ibuprofen after PT in SC-mice can rescue sensory learning up to control levels, but this was not true for OD-plasticity. The effect of partially preserved sensory learning after PT in our EE-animals might be therefore explained by reduced inflammation due to EE-housing. However, ibuprofen acts on the COX pathway of inflammation. So far, no studies are available on the effect of EE on COX-levels in the brain. The effects on sensory learning and OD-plasticity mediated respectively through the COX (ibuprofen) or interleukin pathway (shown to be affected by EE-housing) might be different. This might explain the preserved OD-plasticity after PT in EE-mice, but not after ibuprofen treatment in SC-mice. Indeed, it has been shown that another inflammatory cytokine, tumor necrosis factor- $\alpha$ (TNF- $\alpha$ ), is involved in OD-plasticity in the developing visual mouse cortex. TNF- $\alpha$ knockout mice showed normal initial loss of deprived-eye 
responses after MD, but the subsequent increase in response to the open eye was absent (Kaneko et al. 2008b). Nevertheless, adult OD-plasticity appeared to be unaffected in TNF- $\alpha$ knockout mice (Ranson et al. 2012). It would be appealing to test the influence of the interleukin pathway in PT-mice in further experiments. To do so, SC-mice with a PT-lesion should be treated with an interleukin-1 inhibitor during the MD-period to see whether ODplasticity is restored. A possible compound, which has also been shown to reduce damage caused by a MCAO in rats, is the interleukin-l receptor antagonist (IL-1RA) (Loddick \& Rothwell 1996; Greenhalgh et al. 2010). Moreover, the IL-1 receptor antagonist was already applied successfully in a clinical trial in acute stroke patients: clinical outcomes 3 months after stroke were better in the treated group than in a placebo group, and no adverse side effects of the drug were observed (Emsley et al. 2005).

\section{MMPs}

Matrix metalloproteinases (MMPs) play a role in plasticity in the healthy as well as in the diseased brain (reviewed in Wright \& Harding 2009; Huntley 2012). A recent study implicated that MMP activity is a crucial component of OD-plasticity in the healthy brain (Spolidoro et al. 2012). What is the role of MMPs in a brain that suffered from stroke? After cerebral ischemia in experimental animal models, as well as after stroke in humans, the expression of many MMPs (especially MMP-2 and MMP-9) is increased (Gasche et al. 1999; Heo et al. 1999; Montaner et al. 2001; Rosell et al. 2006). Inhibiting MMP-9 reduced infarct size after MCAO-stroke in rats (Romanic et al. 1998) and blocked cell death after MCAO in mice (Gu et al. 2005). Moreover, MMP-9 knockout mice had significantly smaller lesions after MCAO (Asahi et al. 2000; reviewed in Candelario-Jalil 2009; Morancho et al. 2010). A recent study showed positive effects of MMP inhibition on experience-dependent plasticity after a photothrombotic stroke in mice, next to the barrel cortex (Cybulska-Klosowicz et al. 2011): Injection of a broad-spectrum MMP inhibitor (FN-439) immediately before a stroke rescued experience-dependent barrel cortex plasticity. The action of MMPs can be also modulated by inflammation (reviewed in Candelario-Jalil 2009; Morancho et al. 2010). The pro-inflammatory cytokines TNF- $\alpha$ and IL- $1 \beta$ produce a significant increase in the production of MMPs (MMP-2, -3 and -9) (Gottschall \& Yu 1995; Kauppinen \& Swanson 2005; Candelario-Jalil et al. 2007). Furthermore, a non-selective COX-inhibitor reduced MMP-3 and -9 expression and activation (Candelario-Jalil et al. 2007). On the other hand, MMPs can also modulate inflammation. MMP-3 and -9 activity can modulate the neuroinflammatory response of cytokines (Kim et al. 2005; Woo et al. 2008). Since EE-housing can reduce inflammation after stroke (Ruscher et al. 2009), this might in turn also reduce MMP levels. 
These studies suggest that enhanced inflammatory activity after stroke can be possibly mediated by an increased MMP production. It would be interesting to test whether treatment with MMP-inhibitors could preserve, or rescue, sensory learning and OD-plasticity after a PT stroke in our model. Moreover, it could be tested whether there is a difference in the MMP expression in SC- and EE-mice with and without a PT-lesion.

\section{Serotonin}

As mentioned before, serotonin can be beneficial for rehabilitation after stroke: $5-\mathrm{HT}_{1 \mathrm{~A}}$ receptor agonists reduced infarct size and neuronal damage after a stroke in rats (Prehn et al. 1991; Prehn et al. 1993; Semkova et al. 1998; Torup et al. 2000) and a clinical trial (FLAME), demonstrated a positive action of the serotonin reuptake inhibitor fluoxetine in patients with ischemic stroke (Chollet et al. 2011). Since it has been shown that EE-housing increases serotonin levels (Baroncelli et al. 2010b) and the expression of the gene for the 5-HT $1 \mathrm{~A}$ receptors (Rasmuson et al. 1998) in non-lesioned rats, the preserved OD-plasticity in our PTexperiments might be explained through that mechanism. In order to test this assumption, serotonin levels could be depleted during the MD-period in lesioned EE-mice. This could be done by an intraperitoneal injection of para-chlorophenylalanine, which inhibits the activity of the serotonin synthetic enzyme tryptophan hydroxylase. This procedure has been shown to deplete serotonin levels in the visual cortex of rats (Matsukawa et al. 2003). If plasticity after PT in our EE-mice is mediated by increased serotonin levels, plasticity should not be preserved any longer after that treatment. Furthermore, it was shown that, after stroke, the neuroprotective effect of the serotonin reuptake inhibitor fluoxetine might be also mediated via its anti-inflammatory action. Lim et al. (2009) found that fluoxetine treatment after MCAO in rats blocked the induction of TNF- $\alpha$, COX-2, and IL-1 $\beta$ genes. These effects were accompanied by a reduction of motor impairment and neurological deficits after stroke. These observations are in perfect accordance with the hypothesis that the preserved OD-plasticity after stroke in EE raised mice could be due to a reduced inflammation.

\subsection{Which EE-components are actually effective?}

EE provides a combination of enhanced voluntary physical activity, social interactions and multi-sensory stimulations. Which EE-component contributes the most to positive effects of EE on plasticity, especially on OD-plasticity as shown in our and other studies (Sale et al. 2007; Baroncelli et al. 2010b; Scali et al. 2012)? Or are the positive effects due to a 
combination of these components? These questions are still part of ongoing research. Answering these questions would be valuable to elaborate protocols for humans based on the components of EE which are the most beneficial for recovery after stroke, or to help in the treatment of cortical plasticity related pathologies. A recent study analyzed the impact of motor, social and sensory components of EE on OD-plasticity in an amblyopia model in rats (Baroncelli et al. 2012). Amblyopia is the most common impairment of visual function affecting one eye in adult humans (reviewed in Holmes \& Clarke 2006). In animal models, amblyopia can be artificially caused by long-term deprivation of inputs from one eye by MD. This causes a loss of visual acuity in the deprived eye and a pronounced OD-shift towards the open eye, which becomes permanent if left untreated (Singer et al. 1980; Prusky et al. 2000c; Sale et al. 2007; Maya Vetencourt et al. 2008; Baroncelli et al. 2012).

Baroncelli et al. (2012) observed that enhanced physical activity by providing running wheels to the animals was sufficient to completely restore visual acuity of the formerly deprived eye, and also the binocularity (OD). The activation of the primary motor cortex due to the running/physical activity might lead to the activation of cross-modal plasticity in V1. A recent study supports this hypothesis: in awake mice, the visually evoked firing rate of V1 neurons was doubled when the animals transitioned from standing still to running on a treadmill (Niell $\&$ Stryker 2010). Moreover, wheel running and treadmill training facilitated spatial learning in rodents (Fordyce \& Farrar 1991; van Praag et al. 1999a; Anderson et al. 2000; Vaynman et al. 2004). Furthermore, physical exercise enhanced hippocampal neurogenesis (van Praag et al. 1999a; van Praag et al. 1999b; Fabel et al. 2003; Farmer et al. 2004), synaptic plasticity (van Praag et al. 1999a; Farmer et al. 2004) and increased the levels of BDNF and other growth factors like the insulin-like growth factor (IGF) (review in Cotman \& Berchtold 2002) in rats and mice. Physical exercise also proved to be beneficial in stroke studies. Running wheel exercise increased the number of newborn neurons generated in the hippocampus after a PT stroke in mice and improved spatiotemporal learning achievement (Morris water maze) (Geibig et al. 2012). In addition, running wheel exercise also allowed long-term functional and cognitive improvement: sensory-motor deficit scores and endurance in the wire-hanging test were improved after MCAO in mice (Gertz et al. 2006). Physical exercise is also neuroprotective: voluntary training on a running wheel or a treadmill for 2 to 3 weeks before a MCAO-stroke, reduced cerebral infarct size and sensory-motor deficits in rodents (Wang et al. 2001; Endres et al. 2003).

EE-housing is also enhances visual stimulation, because it provides more visual patterns than a SC. Baroncelli et al. (2012) exposed rats to an enhanced visual stimulation (visual 
enrichment) by placing them into a backlit rotating drum, showing visual stimuli with different spatial frequencies and orientations. This visual enrichment could completely restore visual acuity and OD. They found the same positive effect when amblyopic rats were engaged in a visual perceptual learning task in a modified version of the visual water task (Prusky et al. 2000b; Sale et al. 2011).

In contrast, enhanced social interaction by housing more rats in a SC, was not capable of recovering amblyopia (Baroncelli et al. 2012). Rosenzweig et al. (1978) already showed before that social grouping alone is insufficient to explain the cerebral effects of EE in rats. In agreement with these results, another recent study found that increased social interaction alone had no positive effect on motor coordination (rotarod rest) and learning abilities (skinner box) in mice (Madroñal et al. 2010).

To sum up, also individual components of the EE can have a positive effect in the nonlesioned brain as well as after stroke. Especially voluntary physical activity is a promising component. It proved to be beneficial as a neuroprotective action before stroke, but also helped with rehabilitation after stroke. Whether in our paradigm running wheel exercise alone is also sufficient to prolong and restore OD-plasticity in non-lesioned mice and to preserve OD-plasticity after a PT-lesion, will be an exciting question for future experiments.

\subsection{Enrichment - from bench to bedside?!}

Since most EE research has been done in rodents, the question arises if and how it can be applied in humans.

A recent study applied it to the youngest ones: enriching the brain in terms of body massage accelerated brain development in infants (Guzzetta et al. 2009). A combination of gentle stroking and massaging was highly effective in increasing the maturation of electroencephalographic activity and visual abilities in rat pups as well as in human infants. This effect outlasted the end of the treatment in healthy preterm babies. These experiments were initially inspired by mice experiments, which showed earlier eye opening as well as accelerated development of the visual system and visual acuity in EE-reared mice (Cancedda et al. 2004; Sale et al. 2004). This was most probably due to higher levels of licking behavior that enriched pups experienced from the adult females. Besides, older studies demonstrated that tactilely stimulated preterm neonates gain more weight per day and have reduced levels of stress hormones (Schanberg \& Field 1987; reviewed in Vickers et al. 2009). This makes 
massaging highly recommendable as an intervention to promote growth and development of preterm and low-birth-weight infants.

A suitable enrichment for elderly people seems to be dancing (Kattenstroth et al. 2012). It has the advantage of combining several key features: dancing activities provide physical exercise, social interaction and cognitive requirements. Further, it can be performed at different levels of expertise and abilities, resulting in high motivation. In a recent study, elderly participants performed a 6 months dance course ( 1 hour peer week). Their cognition, intelligence, attention, reaction time, motor, tactile, and postural performance, as well as their subjective well-being was measured in a pre/post-design (Kattenstroth et al. 2012). After those 6 months of dancing class, beneficial effects were found for dance-related parameters such as posture and reaction times, but also for cognitive, tactile, motor performance, and subjective wellbeing. These data suggest that even moderate doses of physical activity in combination with rich sensorimotor, cognitive and social stimulation can contribute to successful aging.

Since most stroke survivors suffer from motor, sensory and cognitive deficits, rehabilitation therapy is essential to regain movement abilities, confidence and independence in everyday life. It has been shown in rodent models that EE promotes motor function recovery and reduces cognitive deficits after stroke. lessen deficits in learning and memory (Ohlsson \& Johansson 1995; Johansson 1996; Biernaskie et al. 2004; Dahlqvist et al. 2004; Rönnbäck et al. 2005; Wurm et al. 2007) (meta-analysis in Janssen et al. (2010)). The concept of environmental enrichment has found its way into rehabilitation therapy thanks to these encouraging results in animal experiments. Very recently a clinical trial was started to test the feasibility of environmental enrichment on stroke patients in rehabilitation (Janssen et al. 2012). The intervention will include different enrichment possibilities for the individual patient as well as shared common activities, like computer, internet, reading material, jigsaw puzzles, board games, music, word and number puzzles. Family members of the stroke patients will be encouraged to join the activities and also contribute hobbies and activities that participants enjoyed before their stroke. Patients should be encouraged to voluntarily utilize provided enrichment possibilities if they are inactive. The main measurement of outcome will be changes in the activity of the patients (physical, cognitive, and social activity). Additional data will be collected before and after the study, including: cognitive function, how independently they can manage their everyday life, mood, boredom and quality of life.

Recently, interactive and virtual reality video gaming emerged as an innovative therapy approach in stroke rehabilitation (reviewed in Laver et al. 2012). The therapy involves the 
usage of computer-based hardware and software that are designed for the patient to engage in environments that appear and feel similar to real world objects and events. This form of intervention has some advantages over conventional therapy approaches. First, the therapist can control easily the difficulty level of the tasks and set it to the appropriate level of challenge according to abilities of the patients. Furthermore, dangerous tasks can be practiced safely and with control, for example crossing a street (Katz et al. 2005). Virtual reality provides an enriched environment with the opportunity to engage in challenging therapeutic tasks with multimodal feedback on performance, for example visual, tactile and audio feedback. Importantly, patients often enjoy the programs more than traditional therapy task and thereby encounter higher numbers of repetitions and spend more time in therapy (Thornton et al. 2005; Bryanton et al. 2006). Although there is a growing interest in virtual reality programs which are specifically designed for rehabilitation, these programs are costly and have been difficult to access. Thus they are rare in rehabilitation clinics. In contrast, interactive gaming consoles are widely available and are increasingly used in clinical settings. Moreover, they can be also used for home training.

To sum up, some beneficial components of EE in animal research can be transferred and adapted to human therapy protocols. EE can be readily applied in humans in many different ways with promising perspectives for enhancing plasticity when necessary, especially in stroke rehabilitation.

\subsection{Conclusion}

Stroke is a major cause of mortality and morbidity. Plasticity is crucial for rehabilitation of the survivors. In this study we analyzed visual plasticity after stroke in an in vivo mouse model using a combination of a behavioral test and optical imaging technique. Our results support the idea that long-ranging interactions play a crucial role for neuronal plasticity that was previously underestimated. V1-sensitivity was probably altered by network interactions, because changes in the major thalamo-cortical inputs were not sufficient to induce ODplasticity in V1. Moreover, inflammation induced by stroke, also affecting the non-lesioned hemisphere, was most likely the cause of diminished sensory learning, but not of impaired OD-plasticity. This further supports the view that distinct cellular mechanisms underlie the two forms of visual plasticity. Consequently, although anti-inflammatory treatment with ibuprofen rescued plasticity in only one of the studied plasticity paradigms, it might prove to be a useful adjuvant therapy to support rehabilitation in the early post-stroke period. Very 
promising targets for the further investigation of plasticity restoration after stroke are the neuromodulatory systems, like the serotonergic, cholinergic and noradrenergic systems, and distinct inflammatory pathways.

EE-housing conditions provide enhanced physical exercise, social interaction, cognitive and sensory stimulation. The present study shows that EE-housing was capable of extending the period for OD-plasticity into late adulthood, with a very pronounced and juvenile-like ODshift. Moreover, EE restored OD-plasticity even after nearly 4 months of standard cage rearing. Our data further partially supports the hypothesis that the EE-effect on OD-plasticity is mediated via a reduction of intracortical inhibitory circuits (Sale et al. 2007; Baroncelli et al. 2010b; Baroncelli et al. 2012). EE also revealed a promising result in photothrombotically lesioned mice: raising mice in EE protected adult mice from stroke-induced impairments of cortical plasticity. Therefore $\mathrm{EE}$ is a potential, non-pharmacological intervention for preserving and restoring plasticity, also after stroke. Which mechanisms mediate the positive effects of EE in our study still needs to be further explored. Possible mechanisms may involve increased serotonergic transmission, increased BDNF levels, increased histone acetylation and/or reduced inflammation. Moreover, it would be exciting to explore whether mice raised in EE will show - even juvenile-like - OD-plasticity their whole life long, and if they will live even longer due to the beneficial effects of EE. It is not only desirable to find the underlying mechanisms of EE, but also to find the most efficient components in order to apply enrichment interventions in humans. For that, running seems to be the most promising component. 


\section{REFERENCES}

Allen Institute for Brain Science (2012) Allen Mouse Brain Atlas [Internet]. http://mouse.brain-map.org/

Alpar A, Gartner U, Hartig W \& Bruckner G (2006) Distribution of pyramidal cells associated with perineuronal nets in the neocortex of rat. Brain Res 1120(1):13-22

Anderson BJ, Rapp DN, Baek DH, McCloskey DP, Coburn-Litvak PS \& Robinson JK (2000) Exercise influences spatial learning in the radial arm maze. Physiol Behav 70(5):425429

Andrews RJ (1991) Transhemispheric diaschisis - a review and comment. Stroke 22(7):943949

Antonini A, Fagiolini M \& Stryker MP (1999) Anatomical correlates of functional plasticity in mouse visual cortex. J Neurosci 19(11):4388-4406

Antonini A \& Stryker MP (1993) Rapid remodeling of axonal arbors in the visual cortex. Science. 260(5115):1819-1821

Antonini A \& Stryker MP (1996) Plasticity of geniculocortical afferents following brief or prolonged monocular occlusion in the cat. J Comp Neurol 369(1):64-82

Artola A \& Singer W (1987) Long-term potentiation and NMDA receptors in rat visual cortex. Nature 330(6149):649-652

Asahi M, Asahi K, Jung JC, del Zoppo GJ, Fini ME \& Lo EH (2000) Role for matrix metalloproteinase 9 after focal cerebral ischemia: effects of gene knockout and enzyme inhibition with BB-94. J Cereb Blood Flow Metab 20(12):1681-1689

Atkinson W, Lockhart SJ, Houghton LA \& Keevil BG (2006) Validation of the measurement of low concentrations of 5-hydroxytryptamine in plasma using high performance liquid chromatography. J Chromatogr B Analyt Technol Biomed Life Sci 832(1):173176

Atwal JK, Pinkston-Gosse J, Syken J, Stawicki S, Wu Y, Shatz C \& Tessier-Lavigne M (2008) PirB is a functional receptor for myelin inhibitors of axonal regeneration. Science. 322(5903):967-970

Baroncelli L, Bonaccorsi J, Milanese M, Bonifacino T, Giribaldi F, Manno I, Cenni MC, Berardi N, Bonanno G, Maffei L \& Sale A (2012) Enriched experience and recovery from amblyopia in adult rats: impact of motor, social and sensory components. Neuropharmacology 62(7):2388-2397

Baroncelli L, Braschi C, Spolidoro M, Begenisic T, Maffei L \& Sale A (2011) Brain plasticity and disease: a matter of inhibition. Neural Plast 2011:286073

Baroncelli L, Braschi C, Spolidoro M, Begenisic T, Sale A \& Maffei L (2010a) Nurturing brain plasticity: impact of environmental enrichment. Cell Death and Differentiation 17(7):1092-1103

Baroncelli L, Sale A, Viegi A, Maya Vetencourt JF, De Pasquale R, Baldini S \& Maffei L (2010b) Experience-dependent reactivation of ocular dominance plasticity in the adult visual cortex. Exp Neurol 226(1):100-109

Barone FC \& Feuerstein GZ (1999) Inflammatory mediators and stroke: new opportunities for novel therapeutics. J Cereb Blood Flow Metab 19(8):819-834

Bartoletti A, Cancedda L, Reid SW, Tessarollo L, Porciatti V, Pizzorusso T \& Maffei L (2002) Heterozygous knock-out mice for brain-derived neurotrophic factor show a pathway-specific impairment of long-term potentiation but normal critical period for monocular deprivation. J Neurosci 22(23):10072-10077

Bartoletti A, Medini P, Berardi N \& Maffei L (2004) Environmental enrichment prevents effects of dark-rearing in the rat visual cortex. Nat Neurosci 7(3):215-216

Bavelier D, Levi DM, Li RW, Dan Y \& Hensch TK (2010) Removing brakes on adult brain plasticity: from molecular to behavioral interventions. J Neurosci 30(45):14964-14971 
Bear MF \& Singer W (1986) Modulation of visual cortical plasticity by acetylcholine and noradrenaline. Nature 320(6058):172-176

Berardi N, Pizzorusso T \& Maffei L (2004) Extracellular matrix and visual cortical plasticity: freeing the synapse. Neuron 44(6):905-908

Bidmon HJ, Oermann E, Schiene K, Schmitt M, Kato K, Asayama K, Witte OW \& Zilles K (2000) Unilateral upregulation of cyclooxygenase-2 following cerebral, cortical photothrombosis in the rat: suppression by MK-801 and co-distribution with enzymes involved in the oxidative stress cascade. J Chem Neuroanat 20(2):163-176

Biernaskie J, Chernenko G \& Corbett D (2004) Efficacy of rehabilitative experience declines with time after focal ischemic brain injury. J Neurosci 24(5):1245-1254

Blasdel GG \& Salama G (1986) Voltage-sensitive dyes reveal a modular organization in monkey striate cortex. Nature 321(6070):579-585

Bliss TV \& Collingridge GL (1993) A synaptic model of memory: long-term potentiation in the hippocampus. Nature 361(6407):31-39

Bliss TV \& Gardner-Medwin AR (1973) Long-lasting potentiation of synaptic transmission in the dentate area of the unanaestetized rabbit following stimulation of the perforant path. J Physiol 232(2):357-374

Bliss TV \& Lomo T (1973) Long-lasting potentiation of synaptic transmission in the dentate area of the anaesthetized rabbit following stimulation of the perforant path. J Physiol 232(2):331-356

Bonhoeffer T \& Grinvald A (1996) Optical Imaging Based on Intrinsic Signals: The Methodology. In Brain Mapping: The Methods. , ed. A Toga, JC Mazziotta, pp. 55-97. San Diego: Academic Press

Boyden ES, Zhang F, Bamberg E, Nagel G \& Deisseroth K (2005) Millisecond-timescale, genetically targeted optical control of neural activity. Nat Neurosci 8(9):1263-1268

Brocher S, Artola A \& Singer W (1992) Agonists of cholinergic and noradrenergic receptors facilitate synergistically the induction of long-term potentiation in slices of rat visual cortex. Brain Res 573(1):27-36

Bruel-Jungerman E, Lucassen PJ \& Francis F (2011) Cholinergic influences on cortical development and adult neurogenesis. Behav Brain Res 221(2):379-388

Bryanton C, Bosse J, Brien M, McLean J, McCormick A \& Sveistrup H (2006) Feasibility, motivation, and selective motor control: virtual reality compared to conventional home exercise in children with cerebral palsy. Cyberpsychol Behav 9(2):123-128

Buchkremer-Ratzmann I, August M, Hagemann G \& Witte OW (1996) Electrophysiological transcortical diaschisis after cortical photothrombosis in rat brain. Stroke 27(6):11051109; discussion 1109-1111

Bundesministerium für Bildung und Forschung (BMBF) (2003) Gesundheitsforschung: Forschung für den Menschen. Newsletter Thema Schlaganfall. Bonn: BMBF

Caleo M, Lodovichi C \& Maffei L (1999) Effects of nerve growth factor on visual cortical plasticity require afferent electrical activity. Eur J Neurosci 11(8):2979-2984

Cancedda L, Putignano E, Sale A, Viegi A, Berardi N \& Maffei L (2004) Acceleration of visual system development by environmental enrichment. J Neurosci 24(20):48404848

Candelario-Jalil E, Taheri S, Yang Y, Sood R, Grossetete M, Estrada EY, Fiebich BL \& Rosenberg GA (2007) Cyclooxygenase inhibition limits blood-brain barrier disruption following intracerebral injection of tumor necrosis factor-alpha in the rat. The Journal of pharmacology and experimental therapeutics 323(2):488-498

Candelario-Jalil E, Yang Y \& Rosenberg GA (2009) Diverse roles of matrix metalloproteinases and tissue inhibitors of metalloproteinases in neuroinflammation and cerebral ischemia. Neuroscience 158(3):983-994

Cang J, Kalatsky VA, Löwel S \& Stryker MP (2005) Optical imaging of the intrinsic signal as a measure of cortical plasticity in the mouse. Vis Neurosci 22(5):685-691 
Carey JR, Kimberley TJ, Lewis SM, Auerbach EJ, Dorsey L, Rundquist P \& Ugurbil K (2002) Analysis of fMRI and finger tracking training in subjects with chronic stroke. Brain 125(Pt 4):773-788

Carulli D, Pizzorusso T, Kwok JCF, Putignano E, Poli A, Forostyak S, Andrews MR, Deepa SS, Glant TT \& Fawcett JW (2010) Animals lacking link protein have attenuated perineuronal nets and persistent plasticity. Brain 133(Pt 8):2331-2347

Chamorro A \& Hallenbeck J (2006) The harms and benefits of inflammatory and immune responses in vascular disease. Stroke 37(2):291-293

Chattopadhyaya B, Di Cristo G, Higashiyama H, Knott GW, Kuhlman SJ, Welker E \& Huang ZJ (2004) Experience and activity-dependent maturation of perisomatic GABAergic innervation in primary visual cortex during a postnatal critical period. $J$ Neurosci 24(43):9598-9611

Cherubini A, Ruggiero C, Polidori MC \& Mecocci P (2005) Potential markers of oxidative stress in stroke. Free Radic Biol Med 39(7):841-852

Choi DW (1992) Excitotoxic cell death. J Neurobiol 23(9):1261-1276

Chollet F, Tardy J, Albucher JF, Thalamas C, Berard E, Lamy C, Bejot Y, Deltour S, Jaillard A, Niclot P, Guillon B, Moulin T, Marque P, Pariente J, Arnaud C \& Loubinoux I (2011) Fluoxetine for motor recovery after acute ischaemic stroke (FLAME): a randomised placebo-controlled trial. Lancet Neurol 10(2):123-130

Cicinelli P, Pasqualetti P, Zaccagnini M, Traversa R, Oliveri M \& Rossini PM (2003) Interhemispheric asymmetries of motor cortex excitability in the postacute stroke stage: a paired-pulse transcranial magnetic stimulation study. Stroke 34(11):26532658

Ciucci F, Putignano E, Baroncelli L, Landi S, Berardi N \& Maffei L (2007) Insulin-like growth factor 1 (IGF-1) mediates the effects of enriched environment (EE) on visual cortical development. PLOS ONE 2(5):e475

Clark SA, Allard T, Jenkins WM \& Merzenich MM (1988) Receptive fields in the bodysurface map in adult cortex defined by temporally correlated inputs. Nature 332(6163):444-445

Clausen F, Hanell A, Israelsson C, Hedin J, Ebendal T, Mir AK, Gram H \& Marklund N (2011) Neutralization of interleukin-1beta reduces cerebral edema and tissue loss and improves late cognitive outcome following traumatic brain injury in mice. Eur $J$ Neurosci 34(1):110-123

Cohen LG, Celnik P, Pascual-Leone A, Corwell B, Falz L, Dambrosia J, Honda M, Sadato N, Gerloff C, Catala MD \& Hallett M (1997) Functional relevance of cross-modal plasticity in blind humans. Nature 389(6647):180-183

Cole DJ, Patel PM, Reynolds L, Drummond JC \& Marcantonio S (1993) Temporary focal cerebral ischemia in spontaneously hypertensive rats: the effect of ibuprofen on infarct volume. The Journal of pharmacology and experimental therapeutics 266(3):17131717

Cook MJ (1965) The anatomy of the laboratory mouse. London: Academic Press

Cotman CW \& Berchtold NC (2002) Exercise: a behavioral intervention to enhance brain health and plasticity. Trends Neurosci 25(6):295-301

Cowey A \& Franzini C (1979) The retinal origin of uncrossed optic nerve fibres in rats and their role in visual discrimination. Exp Brain Res 35(3):443-455

Cybulska-Klosowicz A, Liguz-Lecznar M, Nowicka D, Ziemka-Nalecz M, Kossut M \& Skangiel-Kramska J (2011) Matrix metalloproteinase inhibition counteracts impairment of cortical experience-dependent plasticity after photothrombotic stroke. Eur J Neurosci 33(12):2238-2246

Dahlqvist P, Rönnbäck A, Bergström SA, Söderström I \& Olsson T (2004) Environmental enrichment reverses learning impairment in the Morris water maze after focal cerebral ischemia in rats. Eur J Neurosci 19(8):2288-2298 
Dailly E, Chenu F, Petit-Demouliere B \& Bourin M (2006) Specificity and efficacy of noradrenaline, serotonin depletion in discrete brain areas of Swiss mice by neurotoxins. J Neurosci Methods 150(1):111-115

Daw NW, Fox K, Sato H \& Czepita D (1992) Critical period for monocular deprivation in the cat visual cortex. J Neurophysiol 67(1):197-202

DeFelipe J (2002) Cortical interneurons: from Cajal to 2001. Prog Brain Res 136:215-238

Di Cristo G, Berardi N, Cancedda L, Pizzorusso T, Putignano E, Ratto GM \& Maffei L (2001) Requirement of ERK activation for visual cortical plasticity. Science. 292(5525):2337-2340

Di Cristo G, Chattopadhyaya B, Kuhlman SJ, Fu Y, Belanger MC, Wu CZ, Rutishauser U, Maffei L \& Huang ZJ (2007) Activity-dependent PSA expression regulates inhibitory maturation and onset of critical period plasticity. Nat Neurosci 10(12):1569-1577

Di Garbo A, Mainardi M, Chillemi S, Maffei L \& Caleo M (2011) Environmental enrichment modulates cortico-cortical interactions in the mouse. PLoS ONE 6(9):e25285

Dietrich WD, Feng ZC, Leistra H, Watson BD \& Rosenthal M (1994) Photothrombotic infarction triggers multiple episodes of cortical spreading depression in distant brain regions. J Cereb Blood Flow Metab 14(1):20-28

Dirnagl U, Iadecola C \& Moskowitz MA (1999) Pathobiology of ischaemic stroke: an integrated view. Trends Neurosci 22(9):391-397

Dityatev A, Frischknecht R \& Seidenbecher CI (2006) Extracellular matrix and synaptic functions. Results and problems in cell differentiation 43:69-97

Domann R, Hagemann G, Kraemer M, Freund HJ \& Witte OW (1993) Electrophysiological changes in the surrounding brain tissue of photochemically induced cortical infarcts in the rat. Neurosci Lett 155(1):69-72

Douglas RM, Alam NM, Silver BD, McGill TJ, Tschetter WW \& Prusky GT (2005) Independent visual threshold measurements in the two eyes of freely moving rats and mice using a virtual-reality optokinetic system. Vis Neurosci 22(5):677-684

Doyere V \& Laroche S (1992) Linear relationship between the maintenance of hippocampal long-term potentiation and retention of an associative memory. Hippocampus 2(1):3948

Dräger UC (1975) Receptive fields of single cells and topography in mouse visual cortex. $J$ Comp Neurol 160(3):269-290

Dräger UC (1978) Observations on monocular deprivation in mice. J Neurophysiol 41(1):2842

Eggermann E \& Jonas P (2012) How the 'slow' Ca(2+) buffer parvalbumin affects transmitter release in nanodomain-coupling regimes. Nat Neurosci 15(1):20-22

Emerson VF, Chalupa LM, Thompson ID \& Talbot RJ (1982) Behavioural, physiological, and anatomical consequences of monocular deprivation in the golden hamster (Mesocricetus auratus). Experimental brain research 45(1-2):168-178

Emsley HC, Smith CJ, Georgiou RF, Vail A, Hopkins SJ, Rothwell NJ \& Tyrrell PJ (2005) A randomised phase II study of interleukin-1 receptor antagonist in acute stroke patients. J Neurol Neurosurg Psychiatry 76(10):1366-1372

Endres M, Gertz K, Lindauer U, Katchanov J, Schultze J, Schrock H, Nickenig G, Kuschinsky W, Dirnagl U \& Laufs U (2003) Mechanisms of stroke protection by physical activity. Ann Neurol 54(5):582-590

Eschenko O, Evrard HC, Neves RM, Beyerlein M, Murayama Y \& Logothetis NK (2012) Tracing of noradrenergic projections using manganese-enhanced MRI. Neuroimage 59(4):3252-3265

Espinosa JS \& Stryker MP (2012) Development and plasticity of the primary visual cortex. Neuron 75(2):230-249 
Estler CJ (1973) Effect of $\alpha$ - and $\beta$-adrenergic blocking agents and para-chlorophenylalanine on morphine- and caffeine-stimulated locomotor activity of mice.

Psychopharmacologia 28(3):261-268

Fabel K, Tam B, Kaufer D, Baiker A, Simmons N, Kuo CJ \& Palmer TD (2003) VEGF is necessary for exercise-induced adult hippocampal neurogenesis. Eur J Neurosci 18(10):2803-2812

Fagiolini M, Fritschy JM, Low K, Mohler H, Rudolph U \& Hensch TK (2004) Specific GABAA circuits for visual cortical plasticity. Science. 303(5664):1681-1683

Fagiolini M \& Hensch TK (2000) Inhibitory threshold for critical-period activation in primary visual cortex. Nature 404(6774):183-186

Fagiolini M, Jensen CL \& Champagne FA (2009) Epigenetic influences on brain development and plasticity. Curr Opin Neurobiol 19(2):207-212

Fagiolini M, Pizzorusso T, Berardi N, Domenici L \& Maffei L (1994) Functional postnatal development of the rat primary visual cortex and the role of visual experience: dark rearing and monocular deprivation. Vision Res 34(6):709-720

Failor S, Nguyen V, Darcy DP, Cang J, Wendland MF, Stryker MP \& McQuillen PS (2010) Neonatal cerebral hypoxia-ischemia impairs plasticity in rat visual cortex. $J$ Neurosci 30(1):81-92

Falchier A, Clavagnier S, Barone P \& Kennedy H (2002) Anatomical evidence of multimodal integration in primate striate cortex. $J$ Neurosci 22(13):5749-5759

Farmer J, Zhao X, van Praag H, Wodtke K, Gage FH \& Christie BR (2004) Effects of voluntary exercise on synaptic plasticity and gene expression in the dentate gyrus of adult male Sprague-Dawley rats in vivo. Neuroscience 124(1):71-79

Feigin VL, Lawes CM, Bennett DA \& Anderson CS (2003) Stroke epidemiology: a review of population-based studies of incidence, prevalence, and case-fatality in the late 20th century. Lancet Neurol 2(1):43-53

Feldman DE (2009) Synaptic Mechanisms for Plasticity in Neocortex. Annu Rev Neurosci 32:33-55

Finger S, Koehler PJ \& Jagella C (2004) The Monakow concept of diaschisis: origins and perspectives. Arch Neurol 61(2):283-288

Fischer A, Sananbenesi F, Wang XY, Dobbin M \& Tsai LH (2007) Recovery of learning and memory is associated with chromatin remodelling. Nature 447(7141):178-182

Fischer QS, Beaver CJ, Yang Y, Rao Y, Jakobsdottir KB, Storm DR, McKnight GS \& Daw NW (2004) Requirement for the RIIbeta isoform of PKA, but not calcium-stimulated adenylyl cyclase, in visual cortical plasticity. J Neurosci 24(41):9049-9058

Fordyce DE \& Farrar RP (1991) Enhancement of spatial learning in F344 rats by physical activity and related learning-associated alterations in hippocampal and cortical cholinergic functioning. Behav Brain Res 46(2):123-133

Frenkel MY \& Bear MF (2004) How monocular deprivation shifts ocular dominance in visual cortex of young mice. Neuron 44(6):917-923

Frostig RD, Xiong Y, Chen-Bee CH, Kvasnak E \& Stehberg J (2008) Large-scale organization of rat sensorimotor cortex based on a motif of large activation spreads. $J$ Neurosci 28(49):13274-13284

Galtrey CM \& Fawcett JW (2007) The role of chondroitin sulfate proteoglycans in regeneration and plasticity in the central nervous system. Brain Research Reviews 54(1):1-18

Garcia JH (1975) The neuropathology of stroke. Hum Pathol 6(5):583-598

Gasche Y, Fujimura M, Morita-Fujimura Y, Copin JC, Kawase M, Massengale J \& Chan PH (1999) Early appearance of activated matrix metalloproteinase-9 after focal cerebral ischemia in mice: a possible role in blood-brain barrier dysfunction. J Cereb Blood Flow Metab 19(9):1020-1028 
Geibig CS, Keiner S \& Redecker C (2012) Functional recruitment of newborn hippocampal neurons after experimental stroke. Neurobiol Dis 46(2):431-439

Gerloff C, Bushara K, Sailer A, Wassermann EM, Chen R, Matsuoka T, Waldvogel D, Wittenberg GF, Ishii K, Cohen LG \& Hallett M (2006) Multimodal imaging of brain reorganization in motor areas of the contralesional hemisphere of well recovered patients after capsular stroke. Brain 129(Pt 3):791-808

Gertz K, Priller J, Kronenberg G, Fink KB, Winter B, Schrock H, Ji S, Milosevic M, Harms C, Bohm M, Dirnagl U, Laufs U \& Endres M (2006) Physical activity improves longterm stroke outcome via endothelial nitric oxide synthase-dependent augmentation of neovascularization and cerebral blood flow. Circ Res 99(10):1132-1140

Gianfranceschi L, Fiorentini A \& Maffei L (1999) Behavioural visual acuity of wild type and bcl2 transgenic mouse. Vision Res 39(3):569-574

Gianfranceschi L, Siciliano R, Walls J, Morales B, Kirkwood A, Huang ZJ, Tonegawa S \& Maffei L (2003) Visual cortex is rescued from the effects of dark rearing by overexpression of BDNF. Proc Natl Acad Sci U S A 100(21):12486-12491

Giolli RA, Blanks RH \& Lui F (2005) The accessory optic system: basic organization with an update on connectivity, neurochemistry, and function. Prog Brain Res 151:407-440

Goetze B, Schmidt KF, Lehmann K, Altrock WD, Gundelfinger ED \& Löwel S (2010) Vision and visual cortical maps in mice with a photoreceptor synaptopathy: reduced but robust visual capabilities in the absence of synaptic ribbons. Neuroimage 49(2):16221631

Gonchar Y, Wang Q \& Burkhalter A (2007) Multiple distinct subtypes of GABAergic neurons in mouse visual cortex identified by triple immunostaining. Front Neuroanat $1: 3$

Gordon JA \& Stryker MP (1996) Experience-dependent plasticity of binocular responses in the primary visual cortex of the mouse. J Neurosci 16(10):3274-3286

Gottschall PE \& Yu X (1995) Cytokines regulate gelatinase A and B (matrix metalloproteinase 2 and 9) activity in cultured rat astrocytes. $J$ Neurochem 64(4):1513-1520

Greenhalgh AD, Galea J, Denes A, Tyrrell PJ \& Rothwell NJ (2010) Rapid brain penetration of interleukin-1 receptor antagonist in rat cerebral ischaemia: pharmacokinetics, distribution, protection. Br J Pharmacol 160(1):153-159

Greifzu F, Schmidt S, Schmidt K-F, Kreikemeier K, Witte OW \& Löwel S (2011) Global impairment and therapeutic restoration of visual plasticity mechanisms after a localized cortical stroke. Proc Natl Acad Sci USA 108(37):15450-15455

Greifzu F, Wolf F \& Löwel S (2012) Network influences on cortical plasticity. e-Neuroforum 2/12:41-48

Grinvald A, Lieke E, Frostig RD, Gilbert CD \& Wiesel TN (1986) Functional architecture of cortex revealed by optical imaging of intrinsic signals. Nature 324(6095):361-364

Grinvald A, Shoham D, Shmuel A, Glaser D, Vanzetta I, Shtoyerman E, Slovin H, Wijnbergen C, Hildesheim R \& Arieli A (1999) In-vivo optical imaging of cortical architecture and dynamics. In Modern Techniques in Neuroscience Research, ed. U Windhorst, H Johansson, pp. 893-969. Berlin Heidelberg: Springer Verlag

Gu Q \& Singer W (1995) Involvement of serotonin in developmental plasticity of kitten visual cortex. Eur J Neurosci 7(6):1146-1153

Gu Z, Cui J, Brown S, Fridman R, Mobashery S, Strongin AY \& Lipton SA (2005) A highly specific inhibitor of matrix metalloproteinase-9 rescues laminin from proteolysis and neurons from apoptosis in transient focal cerebral ischemia. J Neurosci 25(27):64016408

Guzzetta A, Baldini S, Bancale A, Baroncelli L, Ciucci F, Ghirri P, Putignano E, Sale A, Viegi A, Berardi N, Boldrini A, Cioni G \& Maffei L (2009) Massage accelerates brain development and the maturation of visual function. J Neurosci 29(18):6042-6051 
Hagemann G, Redecker C, Neumann-Haefelin T, Freund HJ \& Witte OW (1998) Increased long-term potentiation in the surround of experimentally induced focal cortical infarction. Ann Neurol 44(2):255-258

Hallum LE, Chen SC, Clotherty SL, Morley JW, Suanung GJ \& Lovell NH (2006) Functional optical imaging of intrinsic signals in cerebral cortex. In Wiley Encyclopedia of Biomedical Engineering, ed. M Akay: John Wiley \& Sons

Hanover JL, Huang ZJ, Tonegawa S \& Stryker MP (1999) Brain-derived neurotrophic factor overexpression induces precocious critical period in mouse visual cortex. $J$ Neurosci 19(22):RC40

Harauzov A, Spolidoro M, DiCristo G, De Pasquale R, Cancedda L, Pizzorusso T, Viegi A, Berardi N \& Maffei L (2010) Reducing intracortical inhibition in the adult visual cortex promotes ocular dominance plasticity. J Neurosci 30(1):361-371

Hartig W, Brauer K \& Bruckner G (1992) Wisteria floribunda agglutinin-labelled nets surround parvalbumin-containing neurons. Neuroreport 3(10):869-872

Hata R, Maeda K, Hermann D, Mies G \& Hossmann KA (2000) Evolution of brain infarction after transient focal cerebral ischemia in mice. J Cereb Blood Flow Metab 20(6):937946

Heimel JA, Hartman RJ, Hermans JM \& Levelt CN (2007) Screening mouse vision with intrinsic signal optical imaging. Eur J Neurosci 25(3):795-804

Hensch TK (2005) Critical period plasticity in local cortical circuits. Nat Rev Neurosci 6(11):877-888

Hensch TK, Fagiolini M, Mataga N, Stryker MP, Baekkeskov S \& Kash SF (1998) Local GABA circuit control of experience-dependent plasticity in developing visual cortex. Science. 282(5393):1504-1508

Heo JH, Lucero J, Abumiya T, Koziol JA, Copeland BR \& del Zoppo GJ (1999) Matrix metalloproteinases increase very early during experimental focal cerebral ischemia. $J$ Cereb Blood Flow Metab 19(6):624-633

Hofer SB, Mrsic-Flogel TD, Bonhoeffer T \& Hübener M (2006a) Lifelong learning: ocular dominance plasticity in mouse visual cortex. Curr Opin Neurobiol 16(4):451-459

Hofer SB, Mrsic-Flogel TD, Bonhoeffer T \& Hübener M (2006b) Prior experience enhances plasticity in adult visual cortex. Nat Neurosci 9(1):127-132

Hofer SB, Mrsic-Flogel TD, Bonhoeffer T \& Hübener M (2009) Experience leaves a lasting structural trace in cortical circuits. Nature 457(7227):313-317

Holmes JM \& Clarke MP (2006) Amblyopia. Lancet 367(9519):1343-1351

Hossmann KA (2008) Cerebral ischemia: Models, methods and outcomes. Neuropharmacology 55(3):257-270

Huang ZJ, Kirkwood A, Pizzorusso T, Porciatti V, Morales B, Bear MF, Maffei L \& Tonegawa $\mathrm{S}$ (1999) BDNF regulates the maturation of inhibition and the critical period of plasticity in mouse visual cortex. Cell 98(6):739-755

Hubel DH \& Wiesel TN (1962) Receptive fields, binocular interaction and functional architecture in the cat's visual cortex. J Physiol 160(1):106-154

Hubel DH \& Wiesel TN (1970) The period of susceptibility to the physiological effects of unilateral eye closure in kittens. J Physiol 206(2):419-436

Hubel DH, Wiesel TN \& Levay S (1977) Plasticity of ocular dominance columns in monkey striate cortex. Philosophical Transactions of the Royal Society of London Series BBiological Sciences 278(961):377-409

Hummel F, Celnik P, Giraux P, Floel A, Wu WH, Gerloff C \& Cohen LG (2005) Effects of non-invasive cortical stimulation on skilled motor function in chronic stroke. Brain 128(Pt 3):490-499

Hummel FC \& Cohen LG (2006) Non-invasive brain stimulation: a new strategy to improve neurorehabilitation after stroke? Lancet Neurol 5(8):708-712 
Hummel FC \& Gerloff C (2012) Transkranielle Hirnstimulation nach Schlaganfall. Nervenarzt 83(8):957-965

Huntley GW (2012) Synaptic circuit remodelling by matrix metalloproteinases in health and disease. Nat Rev Neurosci 13(11):743-757

Huopaniemi L, Keist R, Randolph A, Certa U \& Rudolph U (2004) Diazepam-induced adaptive plasticity revealed by alpha1 GABAA receptor-specific expression profiling. J Neurochem 88(5):1059-1067

Ickes BR, Pham TM, Sanders LA, Albeck DS, Mohammed AH \& Granholm AC (2000) Long-term environmental enrichment leads to regional increases in neurotrophin levels in rat brain. Exp Neurol 164(1):45-52

Issa NP, Trachtenberg JT, Chapman B, Zahs KR \& Stryker MP (1999) The critical period for ocular dominance plasticity in the ferret's visual cortex. J Neurosci 19(16):6965-6978

Jablonka J, Kazmierczak M \& Mazurkiewicz P (2012a) Post stroke experience-dependent plasticity impairment depends on interhemispheric interactions. FENS Forum Abstr. 2012. 4855

Jablonka JA, Kossut M, Witte OW \& Liguz-Lecznar M (2012b) Experience-dependent brain plasticity after stroke: effect of ibuprofen and poststroke delay. Eur J Neurosci 36(5):2632-2639

Jablonka JA, Witte OW \& Kossut M (2007) Photothrombotic infarct impairs experiencedependent plasticity in neighboring cortex. Neuroreport 18(2):165-169

Janssen H, Ada L, Karayanidis F, Drysdale K, McElduff P, Pollack M, White J, Nilsson M, Bernhardt J \& Spratt NJ (2012) Translating the use of an enriched environment poststroke from bench to bedside: study design and protocol used to test the feasibility of environmental enrichment on stroke patients in rehabilitation. Int J Stroke 7(6):521526

Janssen H, Bernhardt J, Collier JM, Sena ES, McElduff P, Attia J, Pollack M, Howells DW, Nilsson M, Calford MB \& Spratt NJ (2010) An enriched environment improves sensorimotor function post-ischemic stroke. Neurorehabil Neural Repair 24(9):802813

Johansson BB (1996) Functional outcome in rats transferred to an enriched environment 15 days after focal brain ischemia. Stroke 27(2):324-326

Kalatsky VA \& Stryker MP (2003) New paradigm for optical imaging: temporally encoded maps of intrinsic signal. Neuron 38(4):529-545

Kameyama K, Sohya K, Ebina T, Fukuda A, Yanagawa Y \& Tsumoto T (2010) Difference in binocularity and ocular dominance plasticity between GABAergic and excitatory cortical neurons. J Neurosci 30(4):1551-1559

Kaneko M, Hanover JL, England PM \& Stryker MP (2008a) TrkB kinase is required for recovery, but not loss, of cortical responses following monocular deprivation. Nat Neurosci 11(4):497-504

Kaneko M, Stellwagen D, Malenka RC \& Stryker MP (2008b) Tumor necrosis factor-alpha mediates one component of competitive, experience-dependent plasticity in developing visual cortex. Neuron 58(5):673-680

Kanold PO, Kim YA, GrandPre T \& Shatz CJ (2009) Co-regulation of ocular dominance plasticity and NMDA receptor subunit expression in glutamic acid decarboxylase-65 knock-out mice. J Physiol 587(Pt 12):2857-2867

Katagiri H, Fagiolini M \& Hensch TK (2007) Optimization of somatic inhibition at critical period onset in mouse visual cortex. Neuron 53(6):805-812

Kattenstroth J-C, Kalisch T, Holt S, Tegenthoff M \& Dinse HR (2012) Six months of dance intervention enhances postural, sensorimotor, and cognitive performance in elderly without affecting cardio-respiratory functions. Frontiers in Aging Neuroscience 5:5 
Katz N, Ring H, Naveh Y, Kizony R, Feintuch U \& Weiss PL (2005) Interactive virtual environment training for safe street crossing of right hemisphere stroke patients with unilateral spatial neglect. Disabil Rehabil 27(20):1235-1243

Kauppinen TM \& Swanson RA (2005) Poly(ADP-ribose) polymerase-1 promotes microglial activation, proliferation, and matrix metalloproteinase-9-mediated neuron death. $J$ Immunol 174(4):2288-2296

Keller GB, Bonhoeffer T \& Hubener M (2012) Sensorimotor mismatch signals in primary visual cortex of the behaving mouse. Neuron 74(5):809-815

Khedr EM, Ahmed MA, Fathy N \& Rothwell JC (2005) Therapeutic trial of repetitive transcranial magnetic stimulation after acute ischemic stroke. Neurology 65(3):466468

Kim YS, Kim SS, Cho JJ, Choi DH, Hwang O, Shin DH, Chun HS, Beal MF \& Joh TH (2005) Matrix metalloproteinase-3: a novel signaling proteinase from apoptotic neuronal cells that activates microglia. J Neurosci 25(14):3701-3711

Kirkwood A, Rozas C, Kirkwood J, Perez F \& Bear MF (1999) Modulation of long-term synaptic depression in visual cortex by acetylcholine and norepinephrine. J Neurosci 19(5):1599-1609

Klausberger T, Roberts JD \& Somogyi P (2002) Cell type- and input-specific differences in the number and subtypes of synaptic GABA(A) receptors in the hippocampus. $J$ Neurosci 22(7):2513-2521

Kojic L, Gu Q, Douglas RM \& Cynader MS (1997) Serotonin facilitates synaptic plasticity in kitten visual cortex: an in vitro study. Brain Res Dev Brain Res 101(1-2):299-304

Kozlowski DA, James DC \& Schallert T (1996) Use-dependent exaggeration of neuronal injury after unilateral sensorimotor cortex lesions. J Neurosci 16(15):4776-4786

Krafft PR, Bailey EL, Lekic T, Rolland WB, Altay O, Tang J, Wardlaw JM, Zhang JH \& Sudlow CL (2012) Etiology of stroke and choice of models. Int J Stroke 7(5):398-406

Kupers R, Beaulieu-Lefebvre M, Schneider FC, Kassuba T, Paulson OB, Siebner HR \& Ptito M (2011) Neural correlates of olfactory processing in congenital blindness. Neuropsychologia 49(7):2037-2044

Kupers R, Pappens M, de Noordhout AM, Schoenen J, Ptito M \& Fumal A (2007) rTMS of the occipital cortex abolishes Braille reading and repetition priming in blind subjects. Neurology 68(9):691-693

Landi S, Sale A, Berardi N, Viegi A, Maffei L \& Cenni MC (2007) Retinal functional development is sensitive to environmental enrichment: a role for BDNF. FASEB J 21(1):130-139

Laver K, George S, Thomas S, Deutsch JE \& Crotty M (2012) Cochrane review: virtual reality for stroke rehabilitation. Eur J Phys Rehabil Med 48(3):523-530

Lee S, Hjerling-Leffler J, Zagha E, Fishell G \& Rudy B (2010) The largest group of superficial neocortical GABAergic interneurons expresses ionotropic serotonin receptors. J Neurosci 30(50):16796-16808

Lehmann K \& Löwel S (2008) Age-dependent ocular dominance plasticity in adult mice. PLoS ONE 3(9):e3120

Lein ES, Hawrylycz MJ, Ao N, Ayres M, Bensinger A, Bernard A, Boe AF, Boguski MS, Brockway KS, Byrnes EJ, Chen L, Chen TM, Chin MC, Chong J, Crook BE, Czaplinska A, Dang CN, Datta S, Dee NR, Desaki AL, Desta T, Diep E, Dolbeare TA, Donelan MJ, Dong HW, Dougherty JG, Duncan BJ, Ebbert AJ, Eichele G, Estin LK, Faber C, Facer BA, Fields R, Fischer SR, Fliss TP, Frensley C, Gates SN, Glattfelder KJ, Halverson KR, Hart MR, Hohmann JG, Howell MP, Jeung DP, Johnson RA, Karr PT, Kawal R, Kidney JM, Knapik RH, Kuan CL, Lake JH, Laramee AR, Larsen KD, Lau C, Lemon TA, Liang AJ, Liu Y, Luong LT, Michaels J, Morgan JJ, Morgan RJ, Mortrud MT, Mosqueda NF, Ng LL, Ng R, Orta GJ, Overly CC, Pak TH, Parry SE, Pathak SD, Pearson OC, Puchalski RB, Riley ZL, Rockett HR, Rowland SA, Royall 
JJ, Ruiz MJ, Sarno NR, Schaffnit K, Shapovalova NV, Sivisay T, Slaughterbeck CR, Smith SC, Smith KA, Smith BI, Sodt AJ, Stewart NN, Stumpf KR, Sunkin SM, Sutram M, Tam A, Teemer CD, Thaller C, Thompson CL, Varnam LR, Visel A, Whitlock RM, Wohnoutka PE, Wolkey CK, Wong VY, Wood M, Yaylaoglu MB, Young RC, Youngstrom BL, Yuan XF, Zhang B, Zwingman TA \& Jones AR (2007) Genome-wide atlas of gene expression in the adult mouse brain. Nature 445(7124):168-176

Lesch KP \& Waider J (2012) Serotonin in the modulation of neural plasticity and networks: implications for neurodevelopmental disorders. Neuron 76(1):175-191

LeVay S, Wiesel TN \& Hubel DH (1980) The development of ocular dominance columns in normal and visually deprived monkeys. J Comp Neurol 191(1):1-51

Levelt CN \& Hübener M (2012) Critical-period plasticity in the visual cortex. Annu Rev Neurosci 35:309-330

Liepert J, Hamzei F \& Weiller C (2000) Motor cortex disinhibition of the unaffected hemisphere after acute stroke. Muscle Nerve 23(11):1761-1763

Liguz-Lecznar M \& Kossut M (2013) Influence of Inflammation on Poststroke Plasticity. Neural Plast 2013(Article ID 258582)

Lim CM, Kim SW, Park JY, Kim C, Yoon SH \& Lee JK (2009) Fluoxetine affords robust neuroprotection in the postischemic brain via its anti-inflammatory effect. $J$ Neurosci Res 87(4):1037-1045

Lim DH, Mohajerani MH, Ledue J, Boyd J, Chen S \& Murphy TH (2012) In vivo LargeScale Cortical Mapping Using Channelrhodopsin-2 Stimulation in Transgenic Mice Reveals Asymmetric and Reciprocal Relationships between Cortical Areas. Front Neural Circuits 6:11

Liu T, Clark RK, McDonnell PC, Young PR, White RF, Barone FC \& Feuerstein GZ (1994) Tumor necrosis factor-alpha expression in ischemic neurons. Stroke 25(7):1481-1488

Liu T, McDonnell PC, Young PR, White RF, Siren AL, Hallenbeck JM, Barone FC \& Feuerstein GZ (1993) Interleukin-1 beta mRNA expression in ischemic rat cortex. Stroke 24(11):1746-1750

Loddick SA \& Rothwell NJ (1996) Neuroprotective effects of human recombinant interleukin-1 receptor antagonist in focal cerebral ischaemia in the rat. J Cereb Blood Flow Metab 16(5):932-940

Lodovichi C, Berardi N, Pizzorusso T \& Maffei L (2000) Effects of neurotrophins on cortical plasticity: same or different? J Neurosci 20(6):2155-2165

Lucas D, Bruns I, Battista M, Mendez-Ferrer S, Magnon C, Kunisaki Y \& Frenette PS (2012) Norepinephrine reuptake inhibition promotes mobilization in mice: potential impact to rescue low stem cell yields. Blood 119(17):3962-3965

Luiten PG, Gaykema RP, Traber J \& Spencer DG, Jr. (1987) Cortical projection patterns of magnocellular basal nucleus subdivisions as revealed by anterogradely transported Phaseolus vulgaris leucoagglutinin. Brain Res 413(2):229-250

Ma YL, Wang HL, Wu HC, Wei CL \& Lee EH (1998) Brain-derived neurotrophic factor antisense oligonucleotide impairs memory retention and inhibits long-term potentiation in rats. Neuroscience 82(4):957-967

Madroñal N, López-Aracil C, Rangel A, del Río JA, Delgado-García JM \& Gruart A (2010) Effects of enriched physical and social environments on motor performance, associative learning, and hippocampal neurogenesis in mice. PLoS ONE 5(6):e11130

Maffei A, Nataraj K, Nelson SB \& Turrigiano GG (2006) Potentiation of cortical inhibition by visual deprivation. Nature 443(7107):81-84

Maffei L, Berardi N, Domenici L, Parisi V \& Pizzorusso T (1992) Nerve growth factor (NGF) prevents the shift in ocular dominance distribution of visual cortical neurons in monocularly deprived rats. J Neurosci 12(12):4651-4662 
Majewska A \& Sur M (2003) Motility of dendritic spines in visual cortex in vivo: changes during the critical period and effects of visual deprivation. Proc Natl Acad Sci U S A 100(26):16024-16029

Mandolesi G, Menna E, Harauzov A, von Bartheld CS, Caleo M \& Maffei L (2005) A role for retinal brain-derived neurotrophic factor in ocular dominance plasticity. Curr Biol 15(23):2119-2124

Manganotti P, Patuzzo S, Cortese F, Palermo A, Smania N \& Fiaschi A (2002) Motor disinhibition in affected and unaffected hemisphere in the early period of recovery after stroke. Clin Neurophysiol 113(6):936-943

Mangini NJ \& Pearlman AL (1980) Laminar distribution of receptive field properties in the primary visual cortex of the mouse. J Comp Neurol 193(1):203-222

Mansur CG, Fregni F, Boggio PS, Riberto M, Gallucci-Neto J, Santos CM, Wagner T, Rigonatti SP, Marcolin MA \& Pascual-Leone A (2005) A sham stimulation-controlled trial of rTMS of the unaffected hemisphere in stroke patients. Neurology 64(10):18021804

Marks PA \& Breslow R (2007) Dimethyl sulfoxide to vorinostat: development of this histone deacetylase inhibitor as an anticancer drug. Nat Biotechnol 25(1):84-90

Mataga N, Mizuguchi Y \& Hensch TK (2004) Experience-dependent pruning of dendritic spines in visual cortex by tissue plasminogen activator. Neuron 44(6):1031-1041

Mataga N, Nagai N \& Hensch TK (2002) Permissive proteolytic activity for visual cortical plasticity. Proc Natl Acad Sci U S A 99(11):7717-7721

Matsukawa M, Nakadate K, Ishihara I \& Okado N (2003) Synaptic loss following depletion of noradrenaline and/or serotonin in the rat visual cortex: a quantitative electron microscopic study. Neuroscience 122(3):627-635

Maulden SA, Gassaway J, Horn SD, Smout RJ \& DeJong G (2005) Timing of initiation of rehabilitation after stroke. Arch Phys Med Rehabil 86(12 Suppl 2):S34-S40

Maya-Vetencourt JF, Baroncelli L, Viegi A, Tiraboschi E, Castren E, Cattaneo A \& Maffei L (2012) IGF-1 restores visual cortex plasticity in adult life by reducing local GABA levels. Neural Plast 2012:250421

Maya Vetencourt JF, Sale A, Viegi A, Baroncelli L, De Pasquale R, O'Leary OF, Castren E \& Maffei L (2008) The antidepressant fluoxetine restores plasticity in the adult visual cortex. Science. 320(5874):385-388

Maya Vetencourt JF, Tiraboschi E, Spolidoro M, Castren E \& Maffei L (2011) Serotonin triggers a transient epigenetic mechanism that reinstates adult visual cortex plasticity in rats. Eur J Neurosci 33(1):49-57

McCormick DA (1992) Neurotransmitter actions in the thalamus and cerebral cortex. J Clin Neurophysiol 9(2):212-223

McCurry CL, Shepherd JD, Tropea D, Wang KH, Bear MF \& Sur M (2010) Loss of Arc renders the visual cortex impervious to the effects of sensory experience or deprivation. Nat Neurosci 13(4):450-457

McGee AW, Yang Y, Fischer QS, Daw NW \& Strittmatter SM (2005) Experience-driven plasticity of visual cortex limited by myelin and Nogo receptor. Science. 309(5744):2222-2226

Merabet LB, Swisher JD, McMains SA, Halko MA, Amedi A, Pascual-Leone A \& Somers DC (2007) Combined activation and deactivation of visual cortex during tactile sensory processing. J Neurophysiol 97(2):1633-1641

Merzenich MM, Kaas JH, Wall JT, Sur M, Nelson RJ \& Felleman DJ (1983) Progression of change following median nerve section in the cortical representation of the hand in areas $3 \mathrm{~b}$ and 1 in adult owl and squirrel monkeys. Neuroscience 10(3):639-665

Metin C, Godement P \& Imbert M (1988) The primary visual cortex in the mouse: receptive field properties and functional organization. Exp Brain Res 69(3):594-612 
Mitchell JA, Akarasereenont P, Thiemermann C, Flower RJ \& Vane JR (1993) Selectivity of nonsteroidal antiinflammatory drugs as inhibitors of constitutive and inducible cyclooxygenase. Proc Natl Acad Sci U S A 90(24):11693-11697

Mittmann T \& Eysel UT (2001) Increased synaptic plasticity in the surround of visual cortex lesions in rats. Neuroreport 12(15):3341-3347

Mohammed AH, Henriksson BG, Soderstrom S, Ebendal T, Olsson T \& Seckl JR (1993) Environmental influences on the central nervous system and their implications for the aging rat. Behav Brain Res 57(2):183-191

Mohammed AH, Zhu SW, Darmopil S, Hjerling-Leffler J, Ernfors P, Winblad B, Diamond MC, Eriksson PS \& Bogdanovic N (2002) Environmental enrichment and the brain. Prog Brain Res 138:109-133

Montaner J, Alvarez-Sabin J, Molina C, Angles A, Abilleira S, Arenillas J, Gonzalez MA \& Monasterio J (2001) Matrix metalloproteinase expression after human cardioembolic stroke: temporal profile and relation to neurological impairment. Stroke 32(8):17591766

Moore RY (1993) Principles of synaptic transmission. Ann N Y Acad Sci 695:1-9

Morales B, Choi SY \& Kirkwood A (2002) Dark rearing alters the development of GABAergic transmission in visual cortex. J Neurosci 22(18):8084-8090

Morancho A, Rosell A, Garcia-Bonilla L \& Montaner J (2010) Metalloproteinase and stroke infarct size: role for anti-inflammatory treatment? Innate Inflammation and Stroke 1207:123-133

Morishita H, Miwa JM, Heintz N \& Hensch TK (2010) Lynx1, a cholinergic brake, limits plasticity in adult visual cortex. Science. 330(6008):1238-1240

Mower AF, Liao DS, Nestler EJ, Neve RL \& Ramoa AS (2002) cAMP/Ca2+ response element-binding protein function is essential for ocular dominance plasticity. $J$ Neurosci 22(6):2237-2245

Mrsic-Flogel TD, Hofer SB, Ohki K, Reid RC, Bonhoeffer T \& Hübener M (2007) Homeostatic regulation of eye-specific responses in visual cortex during ocular dominance plasticity. Neuron 54(6):961-972

Murase N, Duque J, Mazzocchio R \& Cohen LG (2004) Influence of interhemispheric interactions on motor function in chronic stroke. Ann Neurol 55(3):400-409

Murphy TH \& Corbett D (2009) Plasticity during stroke recovery: from synapse to behaviour. Nature Reviews Neuroscience 10(12):861-872

Nagel G, Szellas T, Huhn W, Kateriya S, Adeishvili N, Berthold P, Ollig D, Hegemann P \& Bamberg E (2003) Channelrhodopsin-2, a directly light-gated cation-selective membrane channel. Proc Natl Acad Sci U S A 100(24):13940-13945

Nagel G, Szellas T, Kateriya S, Adeishvili N, Hegemann P \& Bamberg E (2005) Channelrhodopsins: directly light-gated cation channels. Biochemical Society Transactions 33:863-866

Neumann-Haefelin T, Hagemann G \& Witte OW (1995) Cellular correlates of neuronal hyperexcitability in the vicinity of photochemically induced cortical infarcts in rats in vitro. Neurosci Lett 193(2):101-104

Niell CM \& Stryker MP (2010) Modulation of visual responses by behavioral state in mouse visual cortex. Neuron 65(4):472-479

Nithianantharajah J \& Hannan AJ (2006) Enriched environments, experience-dependent plasticity and disorders of the nervous system. Nat Rev Neurosci 7(9):697-709

Nudo RJ (2003a) Adaptive plasticity in motor cortex: implications for rehabilitation after brain injury. $J$ Rehabil Med 41 Suppl:7-10

Nudo RJ (2003b) Retuning the misfiring brain. Proc Natl Acad Sci U S A 100(13):7425-7427

Ohlsson AL \& Johansson BB (1995) Environment influences functional outcome of cerebral infarction in rats. Stroke 26(4):644-649 
Oray S, Majewska A \& Sur M (2004) Dendritic spine dynamics are regulated by monocular deprivation and extracellular matrix degradation. Neuron 44(6):1021-1030

Paperna T \& Malach R (1991) Patterns of sensory intermodality relationships in the cerebral cortex of the rat. J Comp Neurol 308(3):432-456

Parepally JM, Mandula H \& Smith QR (2006) Brain uptake of nonsteroidal anti-inflammatory drugs: ibuprofen, flurbiprofen, and indomethacin. Pharm Res 23(5):873-881

Patel PM, Drummond JC, Sano T, Cole DJ, Kalkman CJ \& Yaksh TL (1993) Effect of ibuprofen on regional eicosanoid production and neuronal injury after forebrain ischemia in rats. Brain Research 614(1-2):315-324

Paxinos G \& Franklin KBJ (2001) The Mouse Brain in Stereotaxic Coordinates. San Diego: Academic Press

Paxinos G \& Watson C (2005) The rat brain in stereotaxic coordinates. Oxford: Elsevier

Pham TA, Graham SJ, Suzuki S, Barco A, Kandel ER, Gordon B \& Lickey ME (2004) A semi-persistent adult ocular dominance plasticity in visual cortex is stabilized by activated CREB. Learn Mem 11(6):738-747

Pham TA, Impey S, Storm DR \& Stryker MP (1999) CRE-mediated gene transcription in neocortical neuronal plasticity during the developmental critical period. Neuron 22(1):63-72

Pietrasanta M, Restani L \& Caleo M (2012) The corpus callosum and the visual cortex: plasticity is a game for two. Neural Plast 2012:838672

Pizzorusso T, Medini P, Berardi N, Chierzi S, Fawcett JW \& Maffei L (2002) Reactivation of ocular dominance plasticity in the adult visual cortex. Science. 298(5596):1248-1251

Pizzorusso T, Medini P, Landi S, Baldini S, Berardi N \& Maffei L (2006) Structural and functional recovery from early monocular deprivation in adult rats. Proc Natl Acad Sci USA 103(22):8517-8522

Ploughman M, Windle V, MacLellan CL, White N, Dore JJ \& Corbett D (2009) Brainderived neurotrophic factor contributes to recovery of skilled reaching after focal ischemia in rats. Stroke 40(4):1490-1495

Porciatti V, Pizzorusso T \& Maffei L (1999) The visual physiology of the wild type mouse determined with pattern VEPs. Vision Res 39(18):3071-3081

Pouratian N \& Toga AW (2002) Optical Imaging Based on Intrinsic Signals. In Brain Mapping: The Methods, ed. A Toga, JC Mazziotta, pp. 97-138. San Diego: Elsevier Science

Prehn JH, Backhauss C, Karkoutly C, Nuglisch J, Peruche B, Rossberg C \& Krieglstein J (1991) Neuroprotective properties of 5-HT1A receptor agonists in rodent models of focal and global cerebral ischemia. Eur J Pharmacol 203(2):213-222

Prehn JH, Welsch M, Backhauss C, Nuglisch J, Ausmeier F, Karkoutly C \& Krieglstein J (1993) Effects of serotonergic drugs in experimental brain ischemia: evidence for a protective role of serotonin in cerebral ischemia. Brain Res 630(1-2):10-20

Prusky GT, Alam NM, Beekman S \& Douglas RM (2004) Rapid quantification of adult and developing mouse spatial vision using a virtual optomotor system. Invest Ophthalmol Vis Sci 45(12):4611-4616

Prusky GT, Alam NM \& Douglas RM (2006) Enhancement of vision by monocular deprivation in adult mice. $J$ Neurosci 26(45):11554-11561

Prusky GT \& Douglas RM (2003) Developmental plasticity of mouse visual acuity. Eur J Neurosci 17(1):167-173

Prusky GT, Reidel C \& Douglas RM (2000a) Environmental enrichment from birth enhances visual acuity but not place learning in mice. Behavioural Brain Research 114(1-2):1115

Prusky GT, West PW \& Douglas RM (2000b) Behavioral assessment of visual acuity in mice and rats. Vision Res 40(16):2201-2209 
Prusky GT, West PW \& Douglas RM (2000c) Experience-dependent plasticity of visual acuity in rats. Eur J Neurosci 12(10):3781-3786

Putignano E, Lonetti G, Cancedda L, Ratto G, Costa M, Maffei L \& Pizzorusso T (2007) Developmental downregulation of histone posttranslational modifications regulates visual cortical plasticity. Neuron 53(5):747-759

Qü M, Buchkremer-Ratzmann I, Schiene K, Schroeter M, Witte OW \& Zilles K (1998) Bihemispheric reduction of GABAA receptor binding following focal cortical photothrombotic lesions in the rat brain. Brain Res 813(2):374-380

Que M, Schiene K, Witte OW \& Zilles K (1999) Widespread up-regulation of N-methyl-Daspartate receptors after focal photothrombotic lesion in rat brain. Neurosci Lett 273(2):77-80

Rampon C \& Tsien JZ (2000) Genetic analysis of learning behavior-induced structural plasticity. Hippocampus 10(5):605-609

Ranson A, Cheetham CEJ, Fox K \& Sengpiel F (2012) Homeostatic plasticity mechanisms are required for juvenile, but not adult, ocular dominance plasticity. Proc Natl Acad Sci USA 109(4):1311-1316

Rao Y, Fischer QS, Yang Y, McKnight GS, LaRue A \& Daw NW (2004) Reduced ocular dominance plasticity and long-term potentiation in the developing visual cortex of protein kinase A RII alpha mutant mice. Eur J Neurosci 20(3):837-842

Rasband WS (1997-2012) U.S. National Institutes of Health, Bethesda, Maryland, USA. ImageJ. imagej.nih.gov/ij/

Rasmuson S, Olsson T, Henriksson BG, Kelly PA, Holmes MC, Seckl JR \& Mohammed AH (1998) Environmental enrichment selectively increases 5-HT1A receptor mRNA expression and binding in the rat hippocampus. Brain Res Mol Brain Res 53(1-2):285290

Restani L, Cerri C, Pietrasanta M, Gianfranceschi L, Maffei L \& Caleo M (2009) Functional masking of deprived eye responses by callosal input during ocular dominance plasticity. Neuron 64(5):707-718

Robert Koch Institut (2006) Gesundheit in Deutschland. In Gesundheitsberichterstattung des Bundes, ed. RK Institut. Berlin

Rockland KS \& Ojima H (2003) Multisensory convergence in calcarine visual areas in macaque monkey. Int J Psychophysiol 50(1-2):19-26

Romanic AM, White RF, Arleth AJ, Ohlstein EH \& Barone FC (1998) Matrix metalloproteinase expression increases after cerebral focal ischemia in rats: inhibition of matrix metalloproteinase-9 reduces infarct size. Stroke 29(5):1020-1030

Rönnbäck A, Dahlqvist P, Svensson PA, Jernas M, Carlsson B, Carlsson LM \& Olsson T (2005) Gene expression profiling of the rat hippocampus one month after focal cerebral ischemia followed by enriched environment. Neurosci Lett 385(2):173-178

Rosell A, Ortega-Aznar A, Alvarez-Sabin J, Fernandez-Cadenas I, Ribo M, Molina CA, Lo EH \& Montaner J (2006) Increased brain expression of matrix metalloproteinase-9 after ischemic and hemorrhagic human stroke. Stroke 37(6):1399-1406

Rosenzweig MR (1966) Environmental complexity, cerebral change, and behavior. Am Psychol 21(4):321-332

Rosenzweig MR, Bennett EL, Hebert M \& Morimoto H (1978) Social grouping cannot account for cerebral effects of enriched environments. Brain Res 153(3):563-576

Rosenzweig MR, Krech D, Bennett EL \& Diamond MC (1962) Effects of environmental complexity and training on brain chemistry and anatomy: a replication and extension. J Comp Physiol Psychol 55:429-437

Ruscher K, Johannesson E, Brugiere E, Erickson A, Rickhag M \& Wieloch T (2009) Enriched environment reduces apolipoprotein $\mathrm{E}(\mathrm{ApoE})$ in reactive astrocytes and attenuates inflammation of the peri-infarct tissue after experimental stroke. J Cereb Blood Flow Metab 29(11):1796-1805 
Sadato N, Pascual-Leone A, Grafman J, Ibanez V, Deiber MP, Dold G \& Hallett M (1996) Activation of the primary visual cortex by Braille reading in blind subjects. Nature 380(6574):526-528

Sairanen T, Carpen O, Karjalainen-Lindsberg ML, Paetau A, Turpeinen U, Kaste M \& Lindsberg PJ (2001) Evolution of cerebral tumor necrosis factor-alpha production during human ischemic stroke. Stroke 32(8):1750-1758

Sale A, De Pasquale R, Bonaccorsi J, Pietra G, Olivieri D, Berardi N \& Maffei L (2011) Visual perceptual learning induces long-term potentiation in the visual cortex. Neuroscience 172:219-225

Sale A, Maya Vetencourt JF, Medini P, Cenni MC, Baroncelli L, De Pasquale R \& Maffei L (2007) Environmental enrichment in adulthood promotes amblyopia recovery through a reduction of intracortical inhibition. Nat Neurosci 10(6):679-681

Sale A, Putignano E, Cancedda L, Landi S, Cirulli F, Berardi N \& Maffei L (2004) Enriched environment and acceleration of visual system development. Neuropharmacology 47(5):649-660

Sara SJ (2009) The locus coeruleus and noradrenergic modulation of cognition. Nat Rev Neurosci 10(3):211-223

Sato M \& Stryker MP (2008) Distinctive features of adult ocular dominance plasticity. $J$ Neurosci 28(41):10278-10286

Sawtell NB, Frenkel MY, Philpot BD, Nakazawa K, Tonegawa S \& Bear MF (2003) NMDA receptor-dependent ocular dominance plasticity in adult visual cortex. Neuron 38(6):977-985

Scali M, Baroncelli L, Cenni MC, Sale A \& Maffei L (2012) A rich environmental experience reactivates visual cortex plasticity in aged rats. Exp Gerontol 47(4):337-341

Schaller B \& Graf R (2004) Cerebral ischemia and reperfusion: the pathophysiologic concept as a basis for clinical therapy. J Cereb Blood Flow Metab 24(4):351-371

Schanberg SM \& Field TM (1987) Sensory deprivation stress and supplemental stimulation in the rat pup and preterm human neonate. Child Dev 58(6):1431-1447

Schiene K, Bruehl C, Zilles K, Qu M, Hagemann G, Kraemer M \& Witte OW (1996) Neuronal hyperexcitability and reduction of GABAA-receptor expression in the surround of cerebral photothrombosis. J Cereb Blood Flow Metab 16(5):906-914

Schindelin J, Arganda-Carreras I, Frise E, Kaynig V, Longair M, Pietzsch T, Preibisch S, Rueden C, Saalfeld S, Schmid B, Tinevez JY, White DJ, Hartenstein V, Eliceiri K, Tomancak P \& Cardona A (2012) Fiji: an open-source platform for biological-image analysis. Nat Methods 9(7):676-682

Schroeter M, Jander S \& Stoll G (2002) Non-invasive induction of focal cerebral ischemia in mice by photothrombosis of cortical microvessels: characterization of inflammatory responses. J Neurosci Methods 117(1):43-49

Schulz R, Gerloff C \& Hummel FC (2013) Non-invasive brain stimulation in neurological diseases. Neuropharmacology 64:579-587

Schwab ME (2010) Functions of Nogo proteins and their receptors in the nervous system. Nat Rev Neurosci 11(12):799-811

Scott NA \& Murphy TH (2012) Hemodynamic responses evoked by neuronal stimulation via channelrhodopsin-2 can be independent of intracortical glutamatergic synaptic transmission. PLoS ONE 7(1):e29859

Seitz RJ, Butefisch CM, Kleiser R \& Homberg V (2004) Reorganisation of cerebral circuits in human ischemic brain disease. Restor Neurol Neurosci 22(3-5):207-229

Semkova I, Wolz P \& Krieglstein J (1998) Neuroprotective effect of 5-HT1A receptor agonist, Bay X 3702, demonstrated in vitro and in vivo. Eur J Pharmacol 359(23):251-260

Shams L \& Kim R (2010) Crossmodal influences on visual perception. Phys Life Rev 7(3):269-284 
Shatz CJ \& Stryker MP (1978) Ocular dominance in layer IV of the cat's visual cortex and the effects of monocular deprivation. J Physiol 281:267-283

Shimizu T, Hosaki A, Hino T, Sato M, Komori T, Hirai S \& Rossini PM (2002) Motor cortical disinhibition in the unaffected hemisphere after unilateral cortical stroke. Brain 125(Pt 8):1896-1907

Silingardi D, Scali M, Belluomini G \& Pizzorusso T (2010) Epigenetic treatments of adult rats promote recovery from visual acuity deficits induced by long-term monocular deprivation. Eur J Neurosci 31(12):2185-2192

Simpson J \& Kelly JP (2012) The effects of isolated and enriched housing conditions on baseline and drug-induced behavioural responses in the male rat. Behav Brain Res 234(2):175-183

Singer W, von Grunau M \& Rauschecker J (1980) Functional amblyopia in kittens with unilateral exotropia. I. Electrophysiological assessment. Experimental brain research. Experimentelle Hirnforschung. Experimentation cerebrale 40(3):294-304

Smith GB \& Bear MF (2010) Bidirectional ocular dominance plasticity of inhibitory networks: recent advances and unresolved questions. Frontiers in Cellular Neuroscience 4(21):1-7

Spolidoro M, Putignano E, Munafo C, Maffei L \& Pizzorusso T (2012) Inhibition of matrix metalloproteinases prevents the potentiation of nondeprived-eye responses after monocular deprivation in juvenile rats. Cereb Cortex 22(3):725-734

Stein BE, London N, Wilkinson LK \& Price DD (1996) Enhancement of perceived visual intensity by auditory stimuli: A psychophysical analysis. Journal of Cognitive Neuroscience 8(6):497-506

Stellwagen D \& Malenka RC (2006) Synaptic scaling mediated by glial TNF-alpha. Nature 440(7087):1054-1059

Sugiyama S, Di Nardo AA, Aizawa S, Matsuo I, Volovitch M, Prochiantz A \& Hensch TK (2008) Experience-dependent transfer of Otx2 homeoprotein into the visual cortex activates postnatal plasticity. Cell 134(3):508-520

Sugiyama S, Prochiantz A \& Hensch TK (2009) From brain formation to plasticity: insights on Otx2 homeoprotein. Dev Growth Differ 51(3):369-377

Sweatt JD (2009) Experience-dependent epigenetic modifications in the central nervous system. Biol Psychiatry 65(3):191-197

Syken J, GrandPre T, Kanold PO \& Shatz CJ (2006) PirB restricts ocular-dominance plasticity in visual cortex. Science. 313(5794):1795-1800

Tagawa Y, Kanold PO, Majdan M \& Shatz CJ (2005) Multiple periods of functional ocular dominance plasticity in mouse visual cortex. Nat Neurosci 8(3):380-388

Taha S, Hanover JL, Silva AJ \& Stryker MP (2002) Autophosphorylation of alphaCaMKII is required for ocular dominance plasticity. Neuron 36(3):483-491

Takeuchi N, Chuma T, Matsuo Y, Watanabe I \& Ikoma K (2005) Repetitive transcranial magnetic stimulation of contralesional primary motor cortex improves hand function after stroke. Stroke 36(12):2681-2686

Thornton M, Marshall S, McComas J, Finestone H, McCormick A \& Sveistrup H (2005) Benefits of activity and virtual reality based balance exercise programmes for adults with traumatic brain injury: perceptions of participants and their caregivers. Brain Inj 19(12):989-1000

Tognini P, Manno I, Bonaccorsi J, Cenni MC, Sale A \& Maffei L (2012) Environmental enrichment promotes plasticity and visual acuity recovery in adult monocular amblyopic rats. PLOS ONE 7(4):e34815

Torup L, Moller A, Sager TN \& Diemer NH (2000) Neuroprotective effect of 8-OH-DPAT in global cerebral ischemia assessed by stereological cell counting. Eur J Pharmacol 395(2):137-141 
Tropea D, Kreiman G, Lyckman A, Mukherjee S, Yu H, Horng S \& Sur M (2006) Gene expression changes and molecular pathways mediating activity-dependent plasticity in visual cortex. Nat Neurosci 9(5):660-668

van Praag H, Christie BR, Sejnowski TJ \& Gage FH (1999a) Running enhances neurogenesis, learning, and long-term potentiation in mice. Proc Natl Acad Sci U S A 96(23):1342713431

van Praag H, Kempermann G \& Gage FH (1999b) Running increases cell proliferation and neurogenesis in the adult mouse dentate gyrus. Nat Neurosci 2(3):266-270

van Praag H, Kempermann G \& Gage FH (2000) Neural consequences of environmental enrichment. Nat Rev Neurosci 1(3):191-198

Van Sluyters RC \& Stewart DL (1974) Binocular neurons of the rabbit's visual cortex: effects of monocular sensory deprivation. Experimental brain research 19(2):196-204

van Versendaal D, Rajendran R, Saiepour MH, Klooster J, Smit-Rigter L, Sommeijer JP, De Zeeuw CI, Hofer SB, Heimel JA \& Levelt CN (2012) Elimination of inhibitory synapses is a major component of adult ocular dominance plasticity. Neuron 74(2):374-383

Vasconcelos N, Pantoja J, Belchior H, Caixeta FV, Faber J, Freire MA, Cota VR, Anibal de Macedo E, Laplagne DA, Gomes HM \& Ribeiro S (2011) Cross-modal responses in the primary visual cortex encode complex objects and correlate with tactile discrimination. Proc Natl Acad Sci U S A 108(37):15408-15413

Vaynman S, Ying Z \& Gomez-Pinilla F (2004) Hippocampal BDNF mediates the efficacy of exercise on synaptic plasticity and cognition. Eur J Neurosci 20(10):2580-2590

Vertes RP (1991) A PHA-L analysis of ascending projections of the dorsal raphe nucleus in the rat. J Comp Neurol 313(4):643-668

Vickers A, Ohlsson A, Lacy JB \& Horsley A (2009) Massage for promoting growth and development of preterm and/or low birth-weight infants. Cochrane Database Syst Rev (2):CD000390

Von Monakow C (1914) Die Lokalisation im Großhirn und der Abbau der Funktion durch Kortikale Herde. Wiesbaden, Germany: J.F. Bergmann. 26-34 pp.

Wagor E, Mangini NJ \& Pearlman AL (1980) Retinotopic organization of striate and extrastriate visual cortex in the mouse. J Comp Neurol 193(1):187-202

Wallace MT, Ramachandran R \& Stein BE (2004) A revised view of sensory cortical parcellation. Proc Natl Acad Sci U S A 101(7):2167-2172

Wang Q, Tang XN \& Yenari MA (2007) The inflammatory response in stroke. $J$ Neuroimmunol 184(1-2):53-68

Wang RY, Yang YR \& Yu SM (2001) Protective effects of treadmill training on infarction in rats. Brain Res 922(1):140-143

Wang X, Siren AL, Liu Y, Yue TL, Barone FC \& Feuerstein GZ (1994) Upregulation of intercellular adhesion molecule 1 (ICAM-1) on brain microvascular endothelial cells in rat ischemic cortex. Brain Res Mol Brain Res 26(1-2):61-68

Ward NS (2004) Functional reorganization of the cerebral motor system after stroke. Curr Opin Neurol 17(6):725-730

Ward NS, Brown MM, Thompson AJ \& Frackowiak RS (2003) Neural correlates of outcome after stroke: a cross-sectional fMRI study. Brain 126(Pt 6):1430-1448

Waterhouse BD, Mihailoff GA, Baack JC \& Woodward DJ (1986) Topographical distribution of dorsal and median raphe neurons projecting to motor, sensorimotor, and visual cortical areas in the rat. J Comp Neurol 249(4):460-476, 478-481

Watson BD, Dietrich WD, Busto R, Wachtel MS \& Ginsberg MD (1985) Induction of reproducible brain infarction by photochemically initiated thrombosis. Ann Neurol 17(5):497-504

Weber M, Talmon S, Schulze I, Boeddinghaus C, Gross G, Schoemaker H \& Wicke KM (2009) Running wheel activity is sensitive to acute treatment with selective inhibitors 
for either serotonin or norepinephrine reuptake. Psychopharmacology (Berl) 203(4):753-762

Wegner F, Hartig W, Bringmann A, Grosche J, Wohlfarth K, Zuschratter W \& Bruckner G (2003) Diffuse perineuronal nets and modified pyramidal cells immunoreactive for glutamate and the GABA(A) receptor alphal subunit form a unique entity in rat cerebral cortex. Exp Neurol 184(2):705-714

Wiesel TN \& Hubel DH (1963a) Effects of visual deprivation on morphology and physiology of cells in the cats lateral geniculate body. J Neurophysiol 26:978-993

Wiesel TN \& Hubel DH (1963b) Single-cell responses in striate cortex of kittens deprived of vision in one eye. $J$ Neurophysiol 26:1003-1017

Witte OW \& Stoll G (1997) Delayed and remote effects of focal cortical infarctions: secondary damage and reactive plasticity. Adv Neurol 73:207-227

Woo MS, Park JS, Choi IY, Kim WK \& Kim HS (2008) Inhibition of MMP-3 or -9 suppresses lipopolysaccharide-induced expression of proinflammatory cytokines and iNOS in microglia. $J$ Neurochem 106(2):770-780

Woolf NJ (1991) Cholinergic systems in mammalian brain and spinal cord. Prog Neurobiol 37(6):475-524

Wright JW \& Harding JW (2009) Contributions of matrix metalloproteinases to neural plasticity, habituation, associative learning and drug addiction. Neural Plast 2009:579382

Wurm F, Keiner S, Kunze A, Witte OW \& Redecker C (2007) Effects of skilled forelimb training on hippocampal neurogenesis and spatial learning after focal cortical infarcts in the adult rat brain. Stroke 38(10):2833-2840

Xu L, Fagan SC, Waller JL, Edwards D, Borlongan CV, Zheng J, Hill WD, Feuerstein G \& Hess DC (2004) Low dose intravenous minocycline is neuroprotective after middle cerebral artery occlusion-reperfusion in rats. BMC Neurol 4:7

Yang Y, Fischer QS, Zhang Y, Baumgartel K, Mansuy IM \& Daw NW (2005) Reversible blockade of experience-dependent plasticity by calcineurin in mouse visual cortex. Nat Neurosci 8(6):791-796

Zeitz KP, Guy N, Malmberg AB, Dirajlal S, Martin WJ, Sun L, Bonhaus DW, Stucky CL, Julius D \& Basbaum AI (2002) The 5-HT3 subtype of serotonin receptor contributes to nociceptive processing via a novel subset of myelinated and unmyelinated nociceptors. J Neurosci 22(3):1010-1019

Zhang F, Aravanis AM, Adamantidis A, de Lecea L \& Deisseroth K (2007) Circuit-breakers: optical technologies for probing neural signals and systems. Nature Reviews Neuroscience 8(8):577-581

Zhang S \& Murphy TH (2007) Imaging the impact of cortical microcirculation on synaptic structure and sensory-evoked hemodynamic responses in vivo. PLoS Biol 5(5): e119 


\section{ABBREVIATIONS}

$\begin{array}{ll}\text { AMPA } & \alpha \text {-Amino-3-hydroxy-5-methyl-4-isoxazolepropionic acid } \\ \text { CBI } & \text { Contralateral bias index } \\ \text { ChR2 } & \text { Channelrhodopsin-2 } \\ \text { COX } & \text { Cyclooxygenase } \\ \text { Crtl1 } & \text { Cartilage link protein 1 } \\ \text { CSPG } & \text { Chondroitinsulfat-proteoglycane } \\ \text { cyc/deg } & \text { Cycles per degree } \\ \text { DZ } & \text { Diazepam } \\ \text { EE } & \text { Enriched environment } \\ \text { EPSC } & \text { excitatory postsynaptic current }\end{array}$

GABA $\gamma$-Aminobutyric acid

Ibu Ibuprofen

LTD Long-term depression

LTP Long-term potentiation

MCAO Middle cerebral artery occlusion

MD Monocular deprivation

MMPs Matrix metalloproteinases

n Number of animals

$\mathrm{N}_{2} \mathrm{O} \quad$ Nitrous oxide

$\mathrm{NaCl} \quad$ Sodium chloride

NMDA N-methyl-D-aspartate

$\mathrm{O}_{2} \quad$ Oxygen 


\begin{tabular}{|c|c|}
\hline OD & Ocular dominance \\
\hline ODI & Ocular dominance index \\
\hline $\mathrm{mg}$ & Milligram \\
\hline $\mathrm{ml}$ & Milliliter \\
\hline $\mathrm{mm} / \mathrm{cm}$ & Millimeter / Centimeter \\
\hline PB & Phosphate buffer \\
\hline PBS & Phosphate buffered saline \\
\hline PD & Postnatal day \\
\hline PFA & Paraformaldehyde \\
\hline PNN & Perineuronal net \\
\hline PT & Photothrombosis \\
\hline PV & Parvalbumin \\
\hline S1 & Primary somatosensory cortex \\
\hline SC & Standard cage \\
\hline V1 & Primary visual cortex \\
\hline $\mathrm{w}$ & Week \\
\hline 5-HT & 5-Hydroxytryptamin, serotonin \\
\hline$\mu \mathrm{m}$ & Micrometer \\
\hline$\%$ & Percent \\
\hline$\% / \mathrm{s}$ & Degrees per second \\
\hline
\end{tabular}




\section{ACKNOWLEDGEMENTS}

Many persons have supported me with their time and professional skills to make this thesis possible. Thank you all!

First of all I would like to thank Prof. Dr. Siegrid Löwel for giving me the opportunity to work on this exciting project in her laboratory, for her support and the many possibilities to get actively involved in the scientific community here and abroad.

I want to thank my thesis committee members Prof. Andrea Antal and Prof. Fred Wolf and the whole examination board for their time and interest in my thesis.

I am fortunate to be a member of the GGNB Sensory and Motor Neuroscience program. I would like to thank GGNB for supporting me financially with travel grants and moreover for offering various valuable method and soft skill courses and opportunities to meet other $\mathrm{PhD}$ students and scientists. Thanks also to the other SMN students and the organizing team for great retreats.

I thank Prof. Dr. O. W. Witte, Dr. Silvio Schmidt and all the other colleagues from the HansBerger-Klinik für Neurologie in Jena for the prosperous cooperation and introducing me to the clinical aspects as well as practical methods of stroke research. Certainly I want to thank Prof. Karl-Friedrich Schmidt for the constant helpful and encouraging support throughout the whole time and Dr. Konrad Lehmann for his valuable help with any problems and for methods training. Moreover, I want to thank all the other labmates in Jena: Klaus, Katja and Anne-Kathrin for having a very good time there.

I thank Prof. Dr. Staiger and Robin Wagener for the possibility to use their microscope facility and getting expert advice. Thanks also go to Justyna (especially for the helpful comments on the manuscript and the encouraging support), Karim, Simon, Susanne, Evgenia, Neethu, Baya, Sophia, Bianka, Matthias and Simone for help when I needed it and the good time we spent together inside and outside the lab.

Many thanks to Susi, Chrissi, Frenze, Kathrin, Steffi and all the others for the friendship and support throughout the time.

Special thanks to my parents and my boyfriend for their unlimited love and support. You may never fully understand what I do, but you never stop trying. 